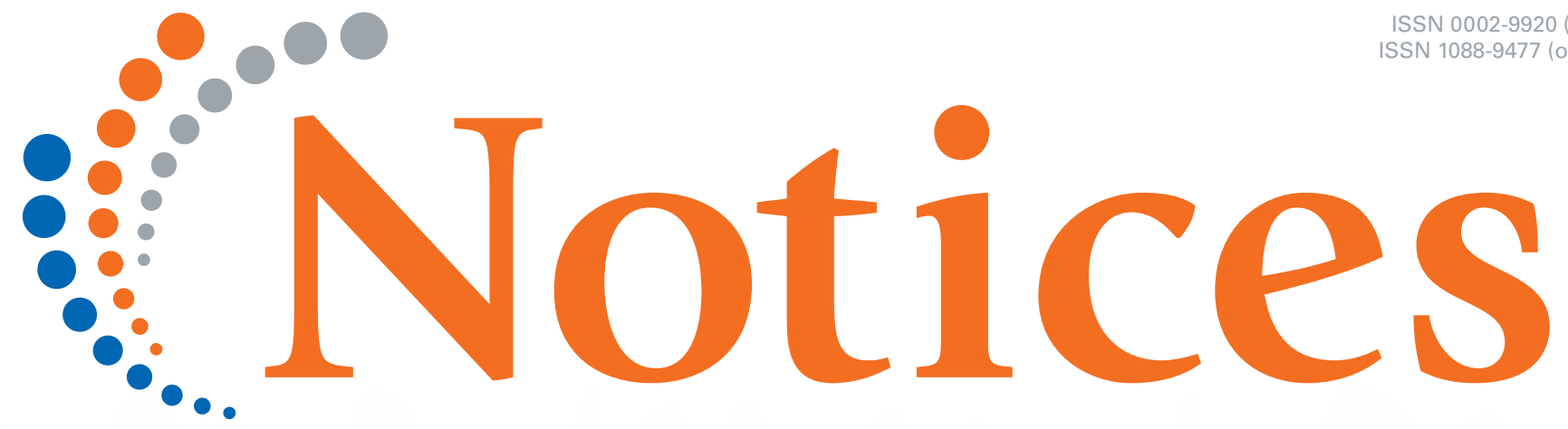

of the American Mathematical Society

January 2019

Volume 66, Number 1

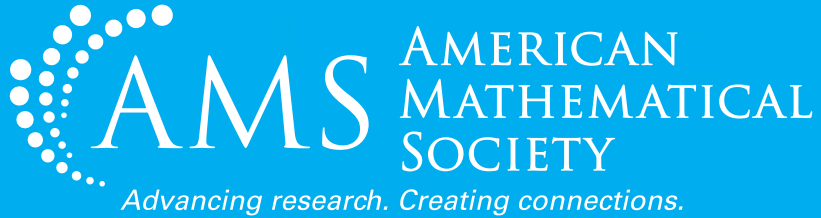

The cover art is from the JMM Sampler, page 84. 


\section{AT THE}

AMS B00TH,

JMM 2019

\section{MEET \& GREET WITH NOTICES EDITOR IN CHIEF ERICA FLAPAN}

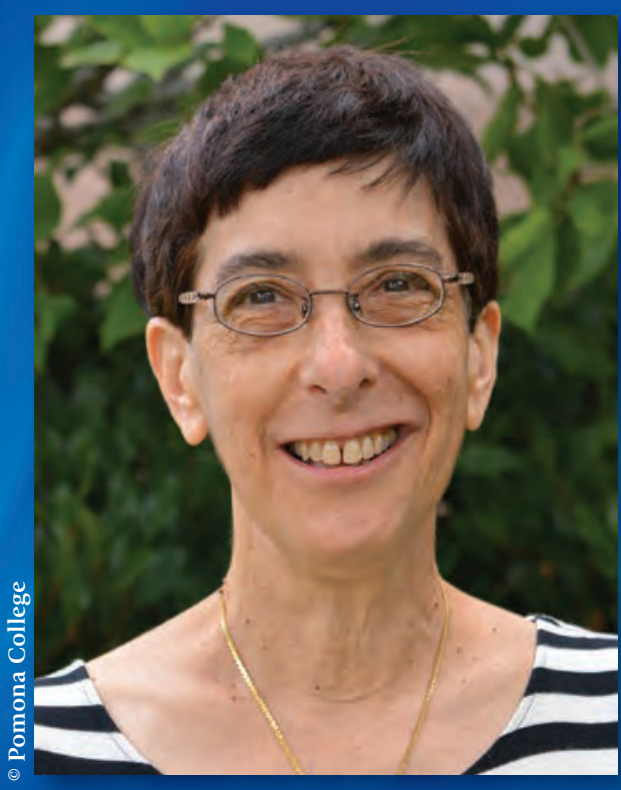

\section{Wednesday | January 16 | 4:00 pm}

Talk to Erica about the AMS membership magazine, pick up a free Notices travel mug*, and enjoy a piece of cake.

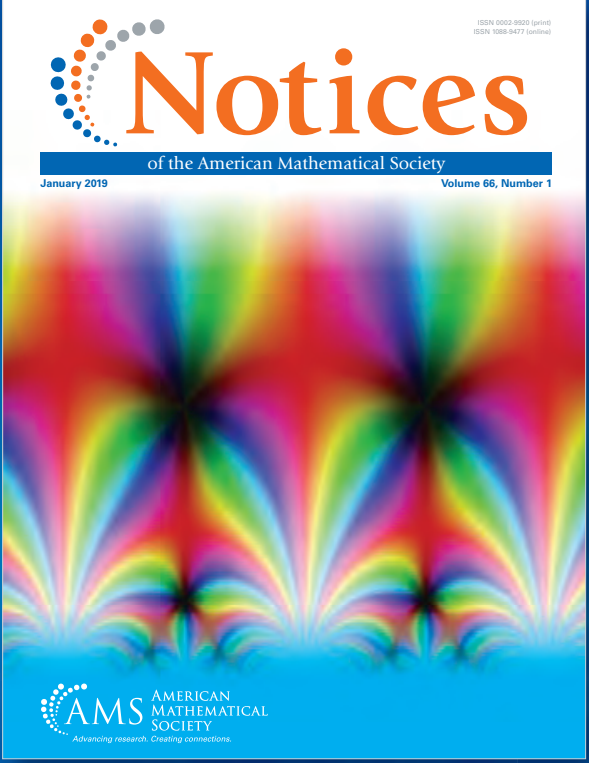




\section{Upcoming Conference}

\section{3th AIMS Conference on Dynamical Systems, Differential Equations and Applications June 5-9, 2020, Atlanta, GA, USA}

The 12th AIMS conference concluded in Taipei on July 9, 2018 with over 1,600 participants and 130 special sessions

AIMS will be attending the Joint Mathematics Meeting (JMM) 2019 as exhibitors. Come by booth 507 to see us!

\section{Electronic Research Announcements - SCI-E}

A journal to announce your research, free of charge ERA was formerly published by the American Mathematical Society before being transferred to AIMS

\section{New Book}

Modelling Disease Ecology with Mathematics (2nd ed.)

By Robert Smith?

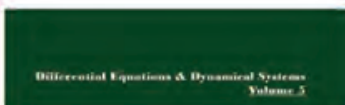

Modelling

Disease Ecology

with.Mathematios

and edilo

Robert Smith?

舟致
AIMS Journals

Advances in Mathematics of Communications

Communications on Pure and Applied Analysis

Discrete and Continuous Dynamical Systems Series A, B \& S

Evolution Equations \& Control Theory

Inverse Problems \& Imaging

Journal of Geometric Mechanics

Journal of Modern Dynamics

Kinetic \& Related Models

Mathematical Biosciences \& Engineering

Mathematical Foundations of Computing

Networks \& Heterogenous Media

...and many more!

Enjoy free online access to all journals for the whole month of January 2019 with code AMS2019 


\section{A WORD FROM...}

Erica Flapan, Notices Editor in Chief

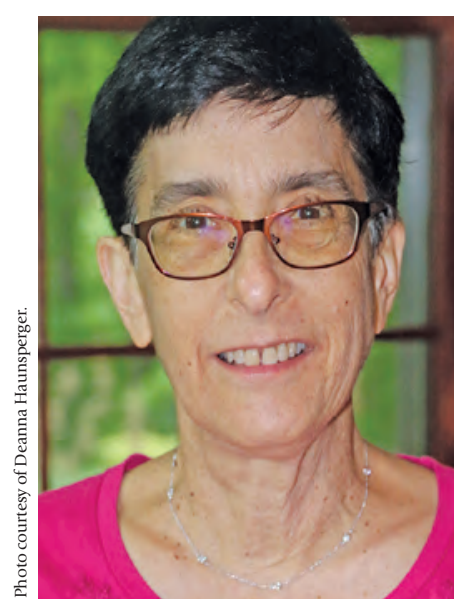

I would like to introduce myself as the new Editor in Chief of the Notices and share my plans with readers. The Notices is an interesting and engaging magazine that is read by mathematicians all over the world. As members of the AMS, we should all be proud to have the Notices as our magazine of record. Personally, I have enjoyed reading the Notices for over 30 years, and I appreciate the opportunity that the AMS has given me to shape the magazine for the next three years. I hope that under my leadership even more people will look forward to reading it each month as much as I do.

Above all, I would like the focus of the Notices to be on expository articles about pure and applied mathematics broadly defined. I would like the authors, topics, and writing styles of these articles to be diverse in every sense except for their desire to explain the mathematics that they love in a clear and engaging way. In addition to articles about mathematics, the Notices will include articles about mathematics education, the history of mathematics, and challenges and opportunities for early career mathematicians.

I will continue many of the outstanding features of the current Notices, build on others, and introduce some quarterly columns that are entirely new. In particular, building on the idea of the Graduate Student Section introduced by my predecessor, the Notices will now have an Early Career Section aimed at graduate students, postdocs, and junior mathematicians thinking about what type of career they would like, as well as those who mentor them. This new section is being spearheaded by Associate Editor Angela Gibney, who has tremendous experience mentoring young mathematicians. In the current issue, she gives readers a glimpse of what to expect in this section throughout this year.

In addition, the Notices will contain the following new quarterly columns:

- "Short Stories" exploring interesting mathematical results that are new or not well known, written by Danny Calegari and Igor Pak.

- "GovMath" showcasing mathematical discoveries that have come out of national laboratories and agencies, edited by Emilie Purvine, who is one of my associate editors and a data scientist at Pacific Northwest National Laboratories.

- "Math Reviews News" presenting what's new from the perspective of Math Reviews, written by Edward Dunne, the Executive Editor of Mathematical Reviews ${ }^{\circledR}$.

- "Washington Update" reporting what the AMS Office of Government Relations is doing to connect the Mathematics Community to decision makers in Washington, DC, written by Karen Saxe, associate executive director, AMS Office of Government Relations.

- "Math Outside the Bubble" exploring mathematics occurring in the arts, literature, film, and other areas where readers might not expect it, written by Sophia D. Merow, special projects editor and Notices assistant.

These columns will occur in different issues throughout the year, starting with GovMath in the current issue. There will also be biannual reports on congressional briefings held by the AMS Office of Government Relations together with the Mathematical Sciences Research Institute, the first of which is in the current issue.

With all of these different types of articles, sections, and columns, I am hoping there will be enough variety in content and style that every reader can find something that interests them in every issue.

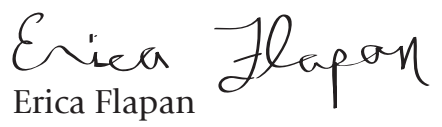

Notices Editor in Chief 


\section{FEATURED}

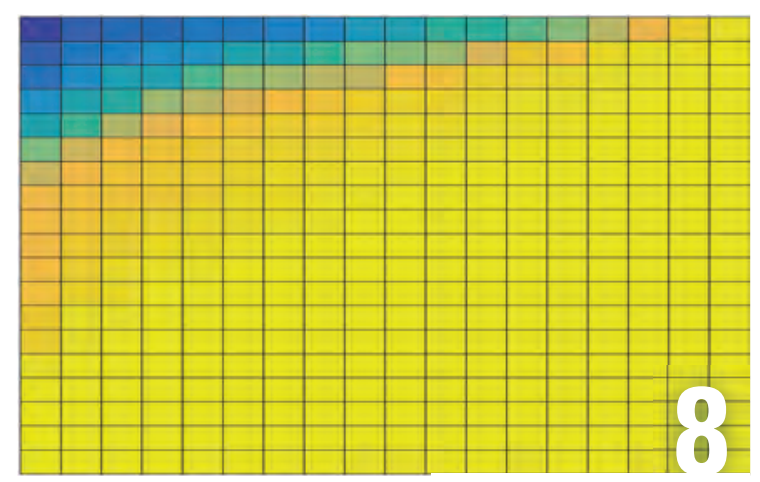

Large Data Analysis and Lyme Disease

Deanna Needell

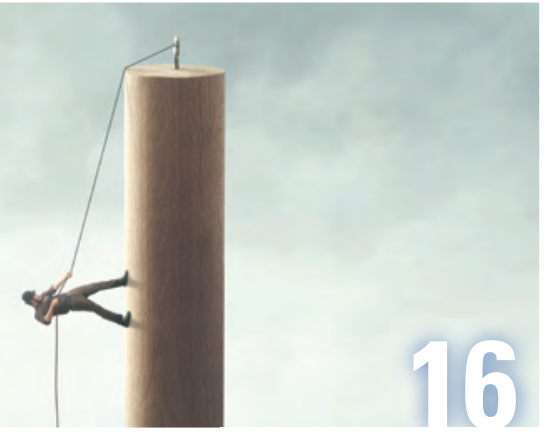

Iwasawa Theory:

A Climb up the Tower

Romyar Sharifi

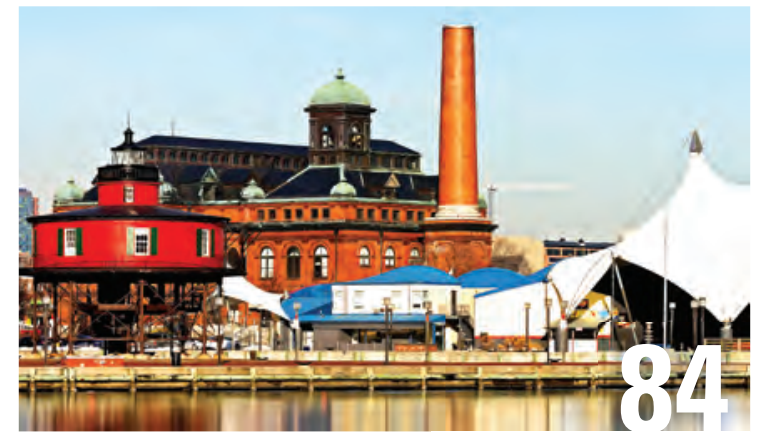

JMM 2019 Sampler

Jesús A. De Loera, Amanda Folsom, Emmanuel Candes, Edray Herber Goins, Bryna Kra, Pamela E. Harris,

Karen Hunger Parshall, Talithia Williams, Lillian B. Pierce, Henok Mawi

\section{ALSO IN THIS ISSUE}

2 A Word from...Erica Flapan

6 Letters to the Editor

Early Career

28 An Introduction to the Section Angela Gibney

$30 \quad$ Journaling Robert Lazarsfeld

Memorial Tributes

32 Alan Baker, 1939-2018

David Masser

37 Michel Raynaud, 1938-2018

Luc Illusie

42 Memories of a Grandmaster: Michel Raynaud and the Flattening by Blowing Up Ahmed Abbes

46 Education: A Survey of Significant Developments in Undergraduate Mathematics Education Over the Past Decade Ron Buckmire

53 Opinion: Mathematics Needs AAAS

Reinhard Laubenbacher

55 Opinion: Venturing Outside the Silo

Hans G. Kaper and Hans Engler

57 AMS Purchase of MAA Press: The View From Acquisitions Sergei Gelfand and Stephen Kennedy

60 WHAT IS.... cryptographic Boolean function? Lilya Budaghyan and Pantelimon Stănică

64 The SASTRA Ramanujan Prize: Its Orgins and Its Winners Krishnaswami Alladi

74 Book Review: A Comprehensive Course in Analysis William T. Ross

78 Bookshelf

79 AMS Bookshelf

80 Fifty Years of CBMS Regional Conferences

David M. Bressoud 


\section{ALSO IN THIS ISSUE, CONT'D}

102 Congressional Briefing: Math Societies to Lawmakers: This Is Why You Should Fund Basic Research

Sophia D. Merow

GovMath

106 Stochastic Resonance When Uncertainty Meets Dynamics

David Barajas-Solano and Zhenyu Huang

107 The Monte Carlo Method:

From Then to Now

J. E. Gubernatis

108 Congressional Fellowship

Margaret D. Callahan

1262018 Annual Meeting of the AAAS

127 General Information Regarding Meetings \& Conferences of the AMS

156 Upcoming in Notices

\section{IN EVERY ISSUE}

110 Community Updates

113 Mathematics People

117 Mathematics Opportunities

118 Early Career Opportunities

119 Classified Advertising

123 New Books Offered by the AMS

129 Meetings and Conferences of the AMS

\section{CURRENT INVITATIONS FROM THE AMS}

27 MRC Research Communities

31 Early Career Mathematicians Membership

45 The Next Generation Fund

73 Call for Applications:

Fan China Exchange Program

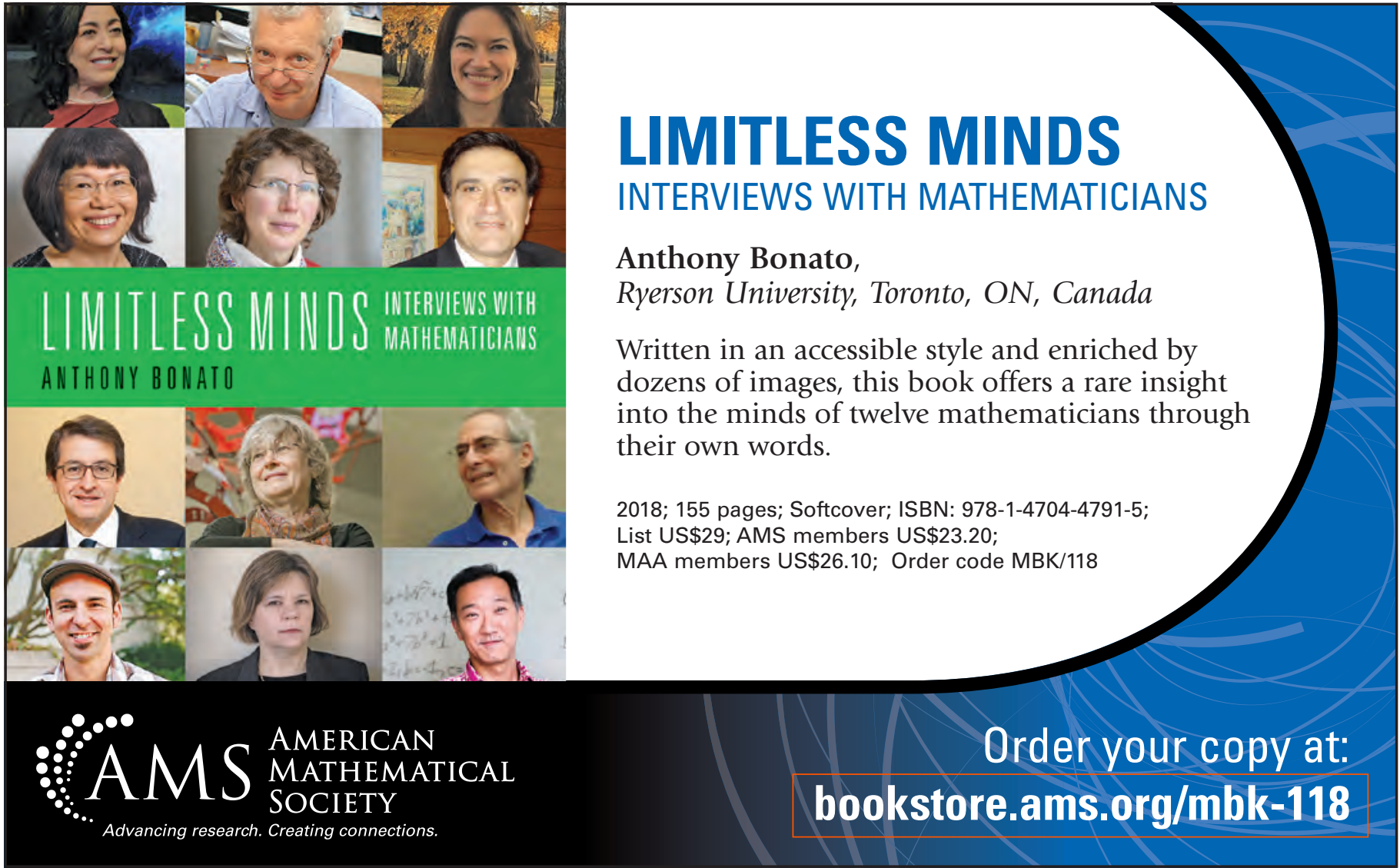




\section{EDITOR IN CHIEF \\ Erica Flapan}

\section{ASSOCIATE EDITORS}

\author{
Dorothy Buck \\ Daniela De Silva \\ Della Dumbaugh \\ Stephan Ramon Garcia \\ Angela Gibney \\ Bryna Kra \\ Daniel Krashen
}

SPECIAL PROJECTS EDITOR and NOTICES ASSISTANT

Sophia D. Merow

\section{CONSULTANTS}

\section{David Jerison Ken Ono}

Jill C. Pipher Kenneth A. Ribet Richard Schoen

MANAGING EDITOR

Rachel L. Rossi

\section{ADVERTISING COORDINATOR}

Anne Newcomb
REPRINT PERMISSIONS
Erin M. Buck

\section{CONTRIBUTING WRITER}

Elaine Kehoe

\section{COMPOSITION, DESIGN, and EDITING}

$\begin{array}{lll}\text { Brain Bartling } & \text { John F. Brady } & \text { Sean M. Downing } \\ \text { Craig Dujon } & \text { Anna Hattoy } & \text { Kerri Malatesta } \\ \text { Lori Nero } & \text { John C. Paul } & \text { Becky Rivard } \\ \text { Courtney Rose } & \text { Mike Southern } & \text { Peter Sykes }\end{array}$

\section{CONTACTING THE NOTICES}

\section{SUBSCRIPTION INFORMATION}

Subscription prices for Volume 65 (2018) are US\$662 list; US $\$ 529.60$ institutional member; US $\$ 397.20$ individual member; US $\$ 595.80$ corporate member. (The subscription price for members is included in the annual dues.) A late charge of $10 \%$ of the subscription price will be imposed upon orders received from non-members after January 1 of the subscription year. Add for postage: Domestic-US\$6.00; InternationalUS $\$ 12.00$. Surface delivery outside the US and India-US\$27; in IndiaUS\$40; expedited delivery to destinations in North America-US\$35; elsewhere-US\$120. Subscriptions and orders for AMS publications should be addressed to the American Mathematical Society, PO Box 845904, Boston, MA 02284-5904 USA. All orders must be prepaid.

\section{ADVERTISING}

Notices publishes situations wanted and classified advertising, and display advertising for publishers and academic or scientific organizations. Advertising requests, materials, and/or questions should be sent to:

\section{classads@ams.org (classified ads)}

notices-ads@ams.org (display ads)

\section{PERMISSIONS}

All requests to reprint Notices articles should be sent to: reprint-permission@ams.org.

\section{SUBMISSIONS}

The editor-in-chief should be contacted about articles for consideration after potential authors have reviewed the "For Authors" page at www.ams.org/publications/journals/notices/noticesauthors

The managing editor should be contacted for additions to our news sections and for any questions or corrections. Contact the managing editor at: notices@ams.org.

Letters to the editor should be sent to: notices-letters@ams.org

To make suggestions for additions to other sections, and for full contact information, see www.ams.org/publications/ journals/notices/noticescontact.
Supported by the AMS membership, most of this publication, including the opportunity to post comments, is freely available electronically through the AMS website, the Society's resource for delivering electronic products and services. Use the URL www.ams.org/notices/ to access the Notices on the website. The online version of the Notices is the version of record, so it may occasionally differ slightly from the print version.
The print version is a privilege of Membership. Graduate students at member institutions can opt to receive the print magazine by updating their individual member profiles at www.ams.org/member-directory. For questions regarding updating your profile, please call 800-321-4267.

For back issues see www.ams.org/backvols. Note: Single issues of the Notices are not available after one calendar year.

[Notices of the American Mathematical Society (ISSN 0002-9920) is published monthly except bimonthly in June/July by the American Mathematical Society at 201 Charles Street, Providence, RI 02904-2213 USA, GST No. 121892046 RT****. Periodicals postage paid at Providence, RI, and additional mailing offices. POSTMASTER: Send address change notices to Notices of the American Mathematical Society, PO Box 6248, Providence, RI 029046248 USA.] Publication here of the Society's street address and the other bracketed information is a technical requirement of the US Postal Service.

\section{(c) Copyright 2019 by the American Mathematical Society. All rights reserved.}

Printed in the United States of America. The paper used in this journal is acid-free and falls within the guidelines established to ensure permanence and durability.

Opinions expressed in signed Notices articles are those of the authors and do not necessarily reflect opinions of the editors or policies of the American Mathematical Society. 


\section{LETTERSTOTHE EDITOR}

\section{Setting the Record Straight}

To the Editor,

We wish to set the record straight on our article, "Seven Characteristics of Successful Calculus Programs, " which appeared in the January, 2015 issue of the Notices. Although only our names appeared as authors of this article, it was in fact, the product of a team effort and was based on a conference report, "Lessons Learned from Case Studies of Successful Calculus Programs at Five Doctoral Degree Granting Institutions," authored by Chris Rasmussen, Jess Ellis (now Jess Ellis Hagman), and Dov Zazkis and presented in 2014 at the 17th Annual Conference on Research in Undergraduate Mathematics Education in Denver, CO, a paper that, unfortunately, was not included in the references to the Notices article.

We especially regret the exclusion of Jess Ellis Hagman as one of the authors of the Notices article and the fact that it has taken so long to correct this error. Jess played an essential role in the data collection, analysis, and writing that led to the identification of the seven features of successful calculus programs, but was not included in the writing of the article that appeared in the Notices. We encourage readers to read and reference the refereed proceedings, which contains more detail on the seven characteristics. It can be found at https://maa.org/ptc under Publications and Reports for Characteristics of Successful Programs in College Calculus.

Sincerely, David Bressoud and Chris Rasmussen

(Received October 16, 2018)

\section{The Back Page}

I enjoy the Notices feature "The Back Page." But I have noticed that sometimes "The Back Page" does not appear on the back page (of the hardcopy Notices) but on some seemingly random page in the middle. Is there a compelling reason for this? If not, then I suggest always printing "The Back Page" on the back page.

-Tim Chow

(Received October 6, 2018)

\footnotetext{
* We invite readers to submit letters to the editor at notices-1etters@ams.org.
}

\section{Regarding the new eligibility criteria for Simons Collaboration Grants}

Let me start by expressing my gratitude to Jim and Marilyn Simons for forming the Simons Foundation, which continues to fund numerous research projects in mathematics and the sciences. The Foundation has a real impact, particularly in the present times when so many in the public discourse either ignore or just plain deny the importance of science.

One of the excellent programs introduced by the Simons Foundation are Collaboration Grants for Mathematicians. While small in comparison to NSF grants, these grants provide substantial and impactful travel support for mathematicians. Collaboration Grants are vitally important for supporting professional activities of mathematicians who otherwise may not have any funding to attend conferences or workshops and who receive minimal support from their home departments.

Since the Simons Foundation is evidently committed to supporting mathematics, it came as a great surprise when the Foundation decided that mathematicians who work in a mathematics department without a $\mathrm{PhD}$ degree granting program are no longer eligible to apply for a Collaboration Grant, as of 2018. This restriction prevents the mathematicians who potentially need support the most, from even applying for a grant.

Throughout my entire professional life, now over 20 years, I have been working in a situation that could be described as "this year the job market is particularly bad." We all know that this has forced many excellent graduates and post-docs to accept positions wherever they could find them, including non-PhD granting departments. By anecdotal evidence, this has affected women even more than men, and has affected professional couples with a two-body problem especially strongly. Many mathematicians who work at non-PhD granting departments maintain strong research programs. This is recognized by the AMS which has recently introduced the AMS Mary P. Dolciani Prize for Excellence in Research "to recognize a mathematician from a department that does not grant a PhD who has an active research program in mathematics and a distinguished record of scholarship, the primary criterion for the prize is an active research program as evidenced by a strong record of peer-reviewed publications." Both the NSF and AWM invite applications for their awards without restrictions on the home institution of applicants. The only criterion for a research award is the quality of candidate's research. Those who produce excellent research, even if they work in a 
less-well-known institution, or while maintaining a higher teaching load, should not be put at a further disadvantage by loss of eligibility for external support for their research.

I recognize that the Simons Foundation is under no obligation to explain any rules that it wishes to implement, and I am grateful for all the impactful support that it provides to the mathematical community. I just respectfully ask that the Simons Foundation reconsider its recent change of eligibility policy for Collaboration Grants, and not automatically exclude researchers who are active and productive despite working at a non-PhD granting department.

\section{-B. Randrianantoanina Miami University randrib@miamioh.edu}

(Received October 28, 2018)

\section{Referees, "Middle Class" Authors, and Ethics}

To referee a math paper is a tough job and, on top of this, it is not paid! As pointed out by Jeremy Avigad in an essay published in the Notices of the AMS (vol. 65 (6) (2018), pg. 681-690) proofs are become longer and to check them "is far less enjoyable than exploring new concepts and ideas". Thus, if asked to referee a 20-pages manuscript, the easiest answer is "the subject seems not to be in the mainstream of the journal..." or something similar.

This is one of the reasons why it is difficult to get published for "middle class" mathematicians and "middle class" means those who are neither super stars nor research beginners. In fact, if you are a super star, journals want to publish what you write because it brings prestige (or impact) for them. If you are a recent $\mathrm{PhD}$ and your supervisor belongs to the editorial board, then the journal will accept what you wrote and they want you to publish because, for supervisors, the success of their students brings also to them prestige and honor.

Add to all this, a lack of ethics in those involved! To substantiate my complaint about lack of ethics, I recall my own experience. In one of my papers, there was an invalid proof of a theorem, although the statement of the theorem was right. Neither I nor the referees noticed that! Only much later did I notice it, when dealing with the same topic: I wrote a two pages corrigendum and sent it to the same journal. The editor-in-chief refused to publish the corrigendum saying that "the journal was a high-quality journal and my 2-pages report did not reach the required level to deserve publication." I wrote back calling his attention to the fact that it was a corrigendum and his last answer was that corrigenda should be sent to the authors of the paper... that is... forget the readers of the journal! —J. M. S. Simões-Pereira
siper@mat.uc.pt

(Received October 8, 2018)
ICERM 2019 Summer Workshops

Arithmetic of Low-dimensional Abelian Varieties June 3-7, 2019 (funded by the Simons Foundation) This workshop will explore a number of themes in the arithmetic of abelian varieties of low dimension, with a focus on computational aspects.

\section{Encrypted Search / June 10-14, 2019}

This workshop will focus on the design and cryptanalysis of practical algorithms and systems that can search on end-to-end encrypted data.

Research Experiences for Undergraduate Faculty (REUF) / June 17-21, 2019

The goals of this workshop are to promote undergraduate research and to forge research collaborations among the participating faculty.

Mathematical Optimization of Systems Impacted by Rare, High-impact Random Events / June 24-28, 2019 This workshop will explore optimization and simulation approaches to designing, planning, and operating systems affected by high-impact rare events.

Perspectives on Dehn Surgery / July 15-19, 2019 This summer school will have courses that unveil Dehn surgery and its suite of techniques to the next generation of researchers in the area.

Women in Data Science and Mathematics Research Collaboration Workshop (WiSDM)

July 29 - August 2, 2019

This research collaboration workshop is targeted toward women working in data science and mathematics.

Applied Mathematical Modeling with Topological Techniques / August 5-9, 2019

This workshop will bring together applied mathematical modeling and applied topology communities, aiming to give modelers exposure to topological techniques still not commonly used in their community.

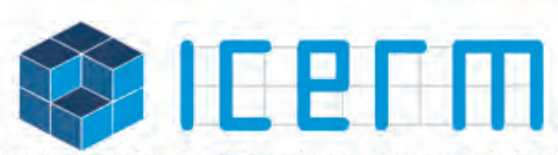

Institute for Computational and Experimental Research in Mathematics
Proposals being accepted: Semester Program

Topical/Hot Topics Workshops Small Group Research Program Summer Undergrad Program

ICERM is a National Science Foundation Mathematics Institute at Brown University in Providence, RI.
Appications being accepted: Semester Program or Workshop Postdoctoral Fellowship Sponsorships being accepted: Academic or Corporate

\section{icerm.brown.edu}




\section{Large Data Analysis and Lyme Disease}

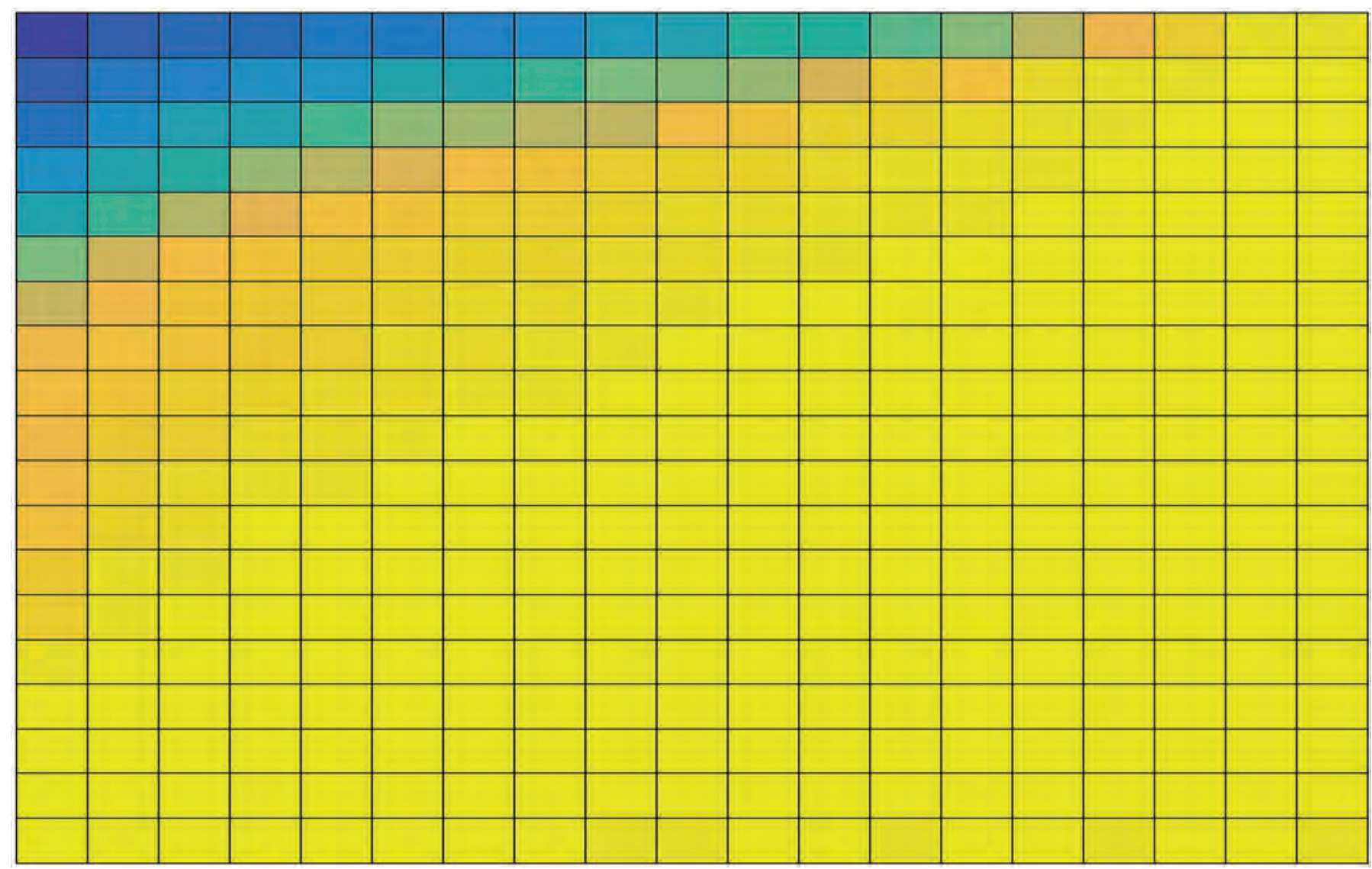

Deanna Needell

\section{Introduction}

Recent advances in technology have led to a monumental increase in large-scale data across many platforms. One may think that more data means more information, but the large-scale nature of modern data actually ends up choking classical analytical methods, making information extraction more challenging than ever before. One mathematical model that has gained a lot of recent attention is the use of sparsity. Sparsity captures the idea that highdimensional signals often contain a very small amount of

Deanna Needell is a professor of mathematics at the University of California, Los Angeles. Her email address is deanna@math. ucla.edu.

Communicated by Notices Associate Editor Noah Simon.

For permission to reprint this article, please contact:

reprint-permission@ams.org.

DOI: https://doi.org/10.1090/noti/1760 intrinsic information. Using this notion, one may design efficient low-dimensional representations of large-scale data as well as robust reconstruction methods for those representations. Moreover, in many applications one does not desire to reconstruct the full signal but rather perform some data analysis task such as classification, clustering, parameter estimation, and feature selection.

Organization. In this article, we will discuss some key mathematical ingredients that can be used for large-scale data analysis. We begin in the section "Signal Reconstruction" with a background to compressive signal processing (CSP), which is used as a foundation and motivation for more recent work in large-scale data analysis. In the section "1-Bit CSP" we discuss the extension of CSP to the 


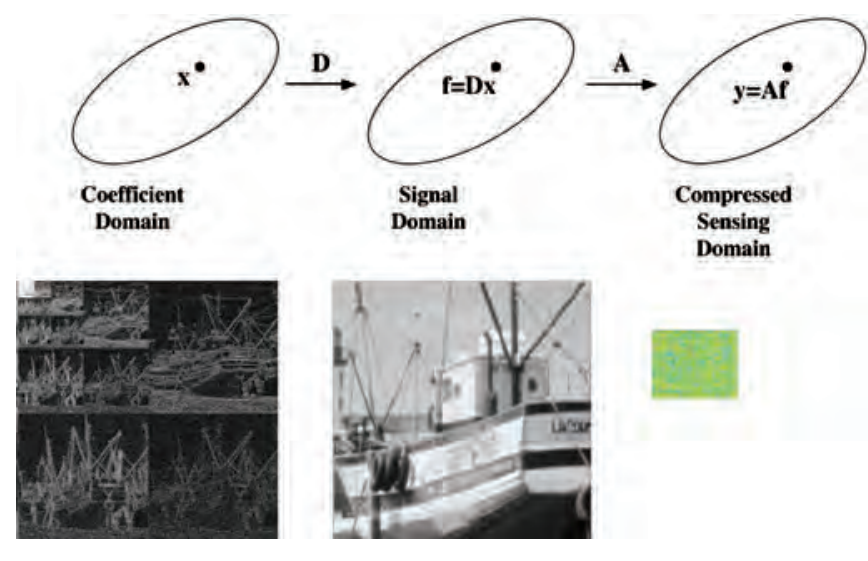

Figure 1. Figure 1. Summary of CSP process.

1-bit version, which utilizes only a single bit per measurement. We relate CSP to the problem of data completion in the section "Data Completion." Finally, the section "Drawing Conclusions from Large-Scale Data" provides an introduction to classification as a simple problem that is motivated by 1-bit CSP. To showcase important challenges with real-world large-scale data, we will use recently acquired Lyme data as a running example throughout. Lyme disease data is especially challenging because of its high variation in patient symptomatology, diagnosis, and treatment, which leads to large-scale but highly incomplete and varied data.

\section{Signal Reconstruction}

In many applications, information about a particular object is acquired in a process called sampling. Sampling is used in medical imaging, analog-to-digital converters, and radar, just to name a few examples. The data objects that we wish to sample are called signals and could be audio signals, images, data files, or any other type of data. Many signals of interest contain far less information than their ambient dimension implies, and such signals are called compressible. Compressive signal processing (CSP) is a recent technology $[7,9]$ that shows these signals can be accurately represented with far fewer samples than traditionally thought. CSP uses the idea of dimension reduction, which reduces the size of compressible signals while still preserving most of their information. There are certain random linear projections that, when applied, provide this type of dimension reduction. Since the problem of recovering a signal from its compressed form is in general illposed (since the resulting mathematical system is highly underdetermined), it is nontrivial to reconstruct a signal from its highly undetermined measurements; we need sophisticated methods to reconstruct the signal from these samples.
Sparsity Model. We will denote our signal of interest by $f \in \mathbb{C}^{n}$. We say that $f$ is $s$-sparse when $f$ has at most $s$ nonzero entries, written $\|f\|_{0} \leq s$. Sparsity plays an important role in CSP because compressible signals are those which are approximated well by sparse signals. In general, a signal can be sparse in this sense or with respect to some orthonormal or overcomplete basis, in which case $f=D x$ for some matrix $D$ and sparse vector $x$. Given a compressible signal, we acquire samples by applying the sampling matrix $A$. The sample vector can then be written as $y=A f+e$, where $e$ is an arbitrary noise vector. The CSP problem is to reconstruct an arbitrary compressible signal $f$ from these noisy samples using a tractable algorithm. That is, given knowledge of the measurements $y$, the measurement matrix $A$, and the sparsifying dictionary $D$, one wishes to (approximately) reconstruct the signal vector $f$. Typically one may assume the sparsity level $s$ is approximately known, although not all methods require such knowledge. The overall process is visualized in Figure 1.

Compressive Signal Processing. Two major approaches for recovery emerged as work in CSP developed. The first method solves an $\ell_{1}$-minimization program to recover the signal, which, when $D$ is the identity, is simply $\operatorname{argmin}_{x}\|x\|_{1}$ s.t. $\|A x-y\|_{2} \leq \varepsilon$, where $\varepsilon$ is the noise tolerance. To analyze this problem, Candès and Tao [6] introduced the restricted isometry property (RIP), which requires the matrix $A$ to be nearly orthonormal on sparse signals [5]. It is now well known that many random $m \times n$ matrices satisfy the RIP with high probability for an optimal number of samples $m \approx s \log n$. Candès et al. show that under the RIP assumption, the $\ell_{1}$-minimization program robustly and accurately recovers sparse signals. The second approach utilizes greedy algorithms that reconstruct the signal iteratively. Greedy algorithms such as IHT and CoSaMP provide both fast runtime and the same optimal recovery guarantees as the optimization-based approach.

\section{1-Bit CSP}

Recovering a signal $x$ from its highly underdetermined image $y=A x$ is already mathematically challenging enough. However, we now take this problem a step further-we ask to keep only the first bit of each entry in $y$ ! One-bit compressed sensing is a new branch of CSP that considers the extreme case when each measurement is quantized to a single bit [2].

In 1-bit CSP, the measurements $y$ are of the form $y_{i}=$ $\operatorname{sign}\left(\left\langle a_{i}, x\right\rangle\right)$, where $a_{i}$ is the $i$ th row of the measurement matrix $A$, and the sign function returns -1 if the input is negative and 1 otherwise. This gives a 1-bit (per entry) representation of the measurement vector $y$. Noise 


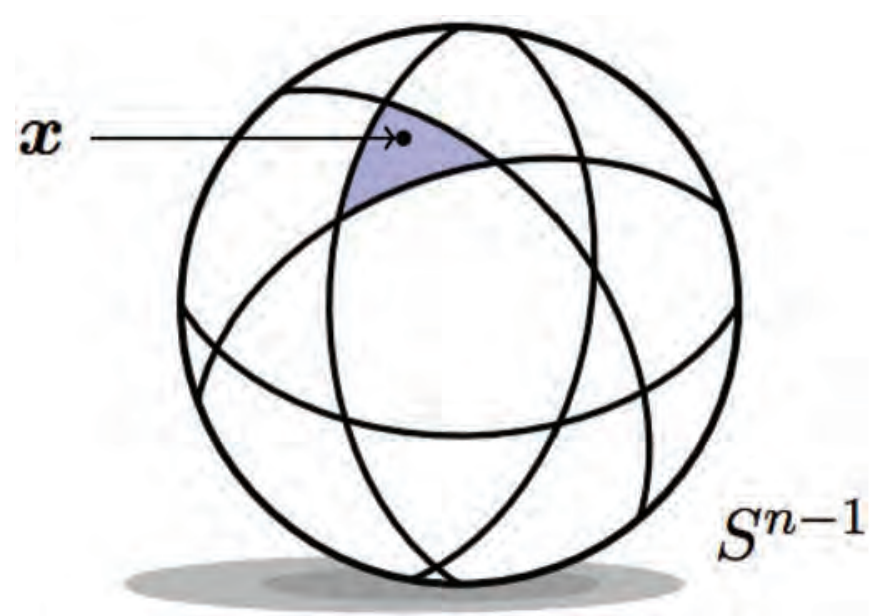

Figure 2. Figure 2. Geometry of 1-bit CSP.

may be added either before or after quantization, the latter type of noise corresponding to a bit-flip. One observation is immediate-there is no way one can ever hope to reconstruct the magnitude of $x$ from such nonlinear measurements. Geometrically, each row of $A$ corresponds to a hyperplane, and the binary measurements $y_{i}$ simply encode on which side of the hyperplane $x$ lies. For example, given the norm of $x$, each measurement divides up the sphere of that radius as in Figure 2. Using this geometry, one can obtain lower bounds on the best possible reconstruction error. Indeed, with this 1-bit model, the best reconstruction $\hat{x}$ from $y$ still must satisfy $\|x-\hat{x}\|_{2} \gtrsim \frac{1}{\lambda}$, where $\lambda=m /(s \log (n / s))$ denotes the oversampling factor (and recall $n$ and $m$ are the original data dimension and compressed dimension, respectively).

However, all hope is not lost. Both issues (the loss of magnitude information and the lower bound on the recovery rate) can be resolved by using dithers, purposefully adding noise to the measurements prior to quantization: $y_{i}=\operatorname{sign}\left(A x+\tau_{i}\right)$, where $\tau_{i}$ is some cleverly chosen scalar noise. Dithers are often used in the theory and practice of analog-to-digital conversion, and one sees that in our case, the value of $\tau_{i}$ simply acts as a new threshold on which to quantize-i.e., $y_{i}$ is now \pm 1 depending on whether it is larger or smaller than $\tau_{i}$. Geometrically, one envisions this as "jiggling" the hyperplanes in such a way as to reveal the norm of the signal $x$. In addition, Baraniuk et al. [1] and Knudson et al. [10] develop both optimization-based and iterative methods that provably provide an exponentially decaying error guarantee of the form $\|x-\hat{x}\|_{2} \lesssim \exp (-\Omega(\lambda))$. In these works, the dithers $\tau_{i}$ are chosen adaptively or set to be i.i.d. Gaussian, depending on the method used for reconstruction.

\section{Data Completion}

Although most data is now extremely large-scale, it is also highly incomplete. State-of-the-art methods like matrix completion allow one to accurately complete a data matrix from observing only a few of its entries, under the assumption that the data is intrinsically low-rank. Generically, the problem is formulated as recovering a matrix $M$ from linear measurements of the form $y_{i}=\left\langle A_{i}, M\right\rangle:=\operatorname{trace}\left(A_{i}^{*} M\right)$ for $i=1 \ldots m$, where $A_{i}$ are matrices of the same dimension as $M$ and where $A^{*}$ denotes the adjoint or conjugate transpose of a matrix $A$. For example, when $A_{i}$ are matrices of all zeros and a single 1 , this corresponds to the classical matrix completion problem when only a subset of entries are observed. In many applications, the underlying complete matrix is approximately low-rank; for example, in collaborative filtering applications like the now-famous Netflix ratings problem, user preferences are accurately described by a small number of variables, and the full set of theoretical ratings is thus very low-rank. One then seeks a low-rank matrix $M$ consistent with these measurements. Since rank minimization is not computationally tractable, one may solve its semi-definite relaxation: $\min _{M}\|M\|_{*}$ s.t. $\left\langle A_{i}, M\right\rangle=y_{i} \forall i$, where $\|M\|_{*}:=\operatorname{trace}\left(\sqrt{M^{*} M}\right)$ denotes the nuclear-norm. It is now well known that when $m$ is on the order of $n r$, nuclear-norm minimization accurately recovers any rank- $r n \times n$ matrix (from, e.g., Gaussian measurements or uniformly at random chosen observations).

Lyme Data. Lyme disease is the most common vectorborne disease in the United States, with CDC estimates of over 300,000 people in the US diagnosed each year. A significant proportion of patients with Lyme disease develop chronic debilitating symptoms, often mimicking other illnesses such as multiple sclerosis (MS) and ALS. Founded over twenty-five years ago, LymeDisease.org (LDo) is a national nonprofit dedicated to advocacy, research, and education. In November 2015, LDo announced the launch of MyLymeData, a patient-powered research project. This data has over 10,000 patients enrolled, includes several phases of initial and follow-up survey responses, and asks patients questions about diagnosis, treatment, symptoms, and quality of life. Like many large-scale surveys, this data is noisy, incomplete, and has a tree-like structure that makes it mathematically challenging. The data used in this work was obtained from the LymeDisease.org patient registry, MyLymeData, Phase 1, June 17, 2017.

Lyme data, like most real-world data, has missing entries that are not random, much less uniformly selected at random. Still, one would like to complete such data matrices so that accurate conclusions can be drawn from the 
complete data, or so that future surveys can be purposefully designed to have missing data (i.e., by not asking every patient every question) to reduce survey fatigue. For example, in the Lyme data, entries may be missing because the user chose not to answer, the survey structure deemed the question irrelevant (i.e., branching), there were corruptions/errors in acquisition, or the survey was designed to ask each patient only a subset of questions. Lee and Shraibman [11] and Foucart et al. [8] recently obtained results (using other methods) exposing recovery guarantees that depend on appropriate parameters of the observation pattern. For example, the latter group analyzed a low-rank projection with de-biasing scheme using a weight matrix $W$ depending on the observation pattern $\Omega$,

$$
\hat{M}=\operatorname{argmin}_{\operatorname{rank} \hat{M} \leq r}\left\|M_{\Omega}-W \odot \hat{M}\right\|^{2},
$$

where $\odot$ denotes the Hadamard (entrywise) product. Note that (1) can be solved very easily by simply using a truncated singular value decomposition (SVD) of $M_{\Omega}$ to find its best rank- $r$ approximation and then multiplying the result entrywise by the reciprocals of the entries in the weight matrix $W$ (assuming they are all nonzero). In other words, the recovered matrix $\hat{M}$ is taken to be the rank- $r$ matrix that best matches the observed entries $M_{\Omega}$, after an appropriate rescaling of the entries. Without such a rescaling, when the sampling pattern is far from uniform, the reconstruction $\hat{M}$ turns out to be very biased, so parts of the matrix need to be scaled by different weights. They also analyze a max-norm $\left(\|X\|_{\max }:=\min _{X=U V^{*}}\|U\|_{2, \infty}\right.$. $\left.\|V\|_{2, \infty}\right)$ projection scheme that tolerates arbitrary nonuniform sampling. Both methods can be applied to matrices with arbitrary sampling patterns; of course, the methods unsurprisingly cannot guarantee accurate completion when the patterns are far from uniform. The results of the former method on the Lyme data are shown in Figure 3. The left plot shows light areas for observed entries and black for unobserved entries; in particular, note that the observation pattern is far from uniform. The middle and right plots show the actual (coded) survey values for the full data matrix (with missing entries) and the completed matrix. Without ground truth for this real data, we cannot provide errors for the completed matrix, but one can validate the method using other statistical techniques.

Structured Observations. Looking again at Figure 3 (left), one immediately notices that the sampling pattern is far from uniform. Indeed, in this type of data and many other applications, the sampling pattern may be deterministic and/or far from uniform. In the Lyme disease survey, missing data arose from patients skipping questions either by survey design or by patient choice. In collaborative filtering, most users do not rate most items whereas a small number of so-called "super-users" rate a large number of
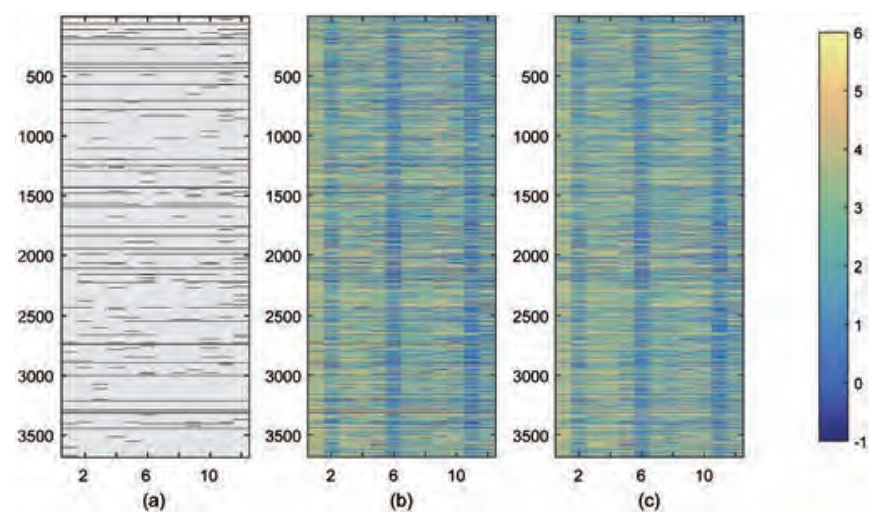

Figure 3. Figure 3. Simple method (1) on the full (incomplete) Lyme symptom data. The actual sampling pattern from the survey for these questions is shown in (a). The full matrix is shown in (b) and the reconstruction in (c).

items. In fact, not only are these sampling patterns nonrandom, the pattern itself yields a lot of untapped information. In both of these examples, most of the unobserved entries are likely due to participant disinterest or irrelevance. This information is useful! Motivated by this setting, we consider a nuclear-norm matrix completion program with an added regularizer that promotes unobserved entries to have small values.

Let $M \in \mathbb{R}^{n_{1} \times n_{2}}$ be the unknown matrix we would like to recover, and let $\Omega$ be the set of indices of the observed entries. Let $\mathcal{P}_{\Omega}: \mathbb{R}^{n_{1} \times n_{2}} \rightarrow \mathbb{R}^{n_{1} \times n_{2}}$, where

$$
\left[\mathcal{P}_{\Omega}\right]_{i j}= \begin{cases}M_{i j} & (i, j) \in \Omega \\ 0 & (i, j) \notin \Omega\end{cases}
$$

as in [3].

Recall the nuclear-norm minimization,

$$
\widehat{M}=\operatorname{argmin}_{A}\|A\|_{*} \text { s.t. } \mathcal{P}_{\Omega}(A)=\mathcal{P}_{\Omega}(M) .
$$

Motivated by applications in which the unobserved entries tend to have small values, we instead solve

$$
\begin{array}{r}
\widetilde{M}=\operatorname{argmin}_{A}\|A\|_{*}+\alpha\left\|\mathcal{P}_{\Omega^{c}}(A)\right\|_{1} \\
\text { s.t. } \mathcal{P}_{\Omega}(A)=\mathcal{P}_{\Omega}(M),
\end{array}
$$

where $\alpha>0$ and the entrywise $L_{1}$-norm $\|M\|_{1}=$ $\sum_{i j}\left|M_{i j}\right|$.

From the Lyme data, we consider a subset of 2,126 patients responding to sixty-five particular questions that gives a fully complete matrix (so we have ground truth for testing purposes). Question responses are integer values between zero and four. Due to computational constraints, for each of the ten trials executed, we randomly sample fifty of these patient surveys to generate a 50 x 65 matrix. We then subsample from the zero and nonzero entries of the data matrix at various rates to generate a matrix 
with missing entries. We complete this subsampled matrix with both (2) and (3) using $L_{1}$ regularization on the unobserved entries and report $\left.\|\widetilde{M}-M\|\right|_{F} / \mid \widehat{M}-M \|_{F}$, averaged over ten trials in Figure 4 . The parameter $\alpha$, for the regularization term, is chosen to be optimal among $\alpha \in$ $\left\{10^{-1}, 10^{-2}, 10^{-3}, 10^{-4}\right\}$. We see, as expected, that when most of the unobserved entries are small and most of the observed entries are large, there is the most improvement in using the regularizer. Preliminary theoretical results can be found in [12], which are motivated by work in robust principal component analysis [4], but future work is needed to clearly quantify the theoretical gains as a function of the sampling rates.

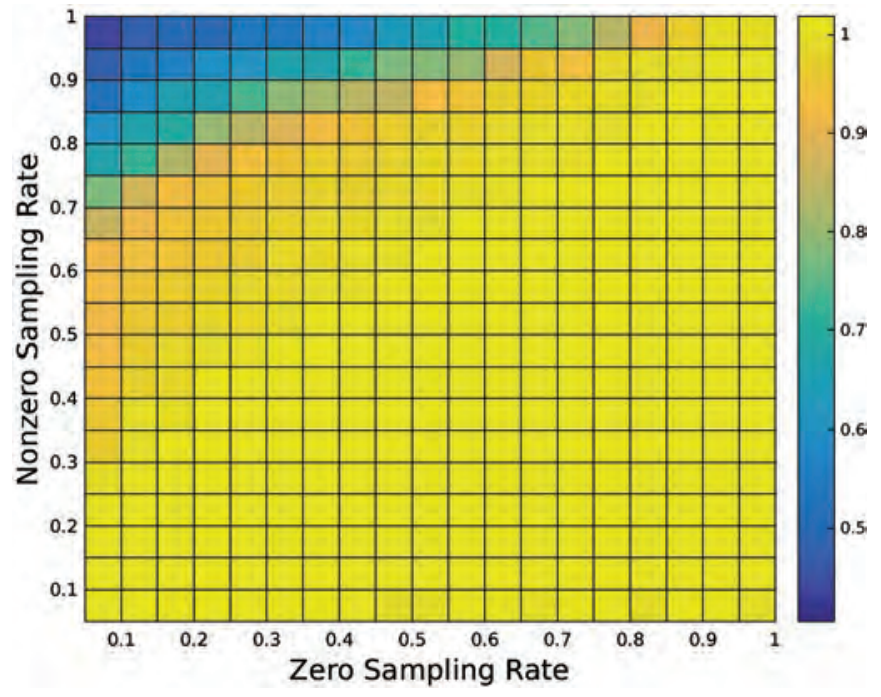

Figure 4. Figure 4. For $\widetilde{M}$ and $\widehat{M}$ given by (3) and (2), respectively, with $L_{1}$ regularization on the recovered values for the unobserved entries, we plot $\|\widetilde{M}-M\|_{F} / \mid \widehat{M}-M \|_{F}$. We consider fifty patient surveys with sixty-five responses each chosen randomly from 2,126 patient surveys. We average results over ten trials and with $\alpha$ optimal among $\alpha \in\left\{10^{-1}, 10^{-2}, 10^{-3}, 10^{-4}\right\}$.

\section{Drawing Conclusions from Large-Scale Data}

Background. In many high-dimensional data applications, one is not interested in just representing the data efficiently or completing missing data, but also in drawing analytical conclusions from that data. Such analytical techniques can be performed on the large-scale data itself or from the efficient representations. Although there are many important data analysis problems, and many sophisticated methods to address them, we focus here on the problem of classification and discuss a simple method motivated by 1-bit CSP that exhibits extremely efficient computation and storage as well as interpretability and mathematical theoretical support.
Classification. Given labeled training data, the classification problem asks to accurately label new unlabeled data. Classification is a canonical problem considered in many areas of statistics and machine learning. Classical approaches include support vector machines (SVM) and logistic regression, and recently there has been a surge in so-called deep learning methods, which rely on learned hidden features through a neural network design. Here, we describe a recent approach put forth by Needell et al. [13] that performs simple and efficient classification from binary data. It will use random hyperplanes and the corresponding binary sign information that provides information about which side of each hyperplane each data point lies. For a new query point, this information will be compared to the same information for all training data, and then a label that best matches the new query point will be assigned. Although motivated by other approaches like SVM and deep learning, this approach is not very comparable, since, e.g., SVM searches for a separating hyperplane as a rule, whereas this approach will utilize and aggregate information from many random hyperplanes.

Let us build some intuition for the approach. Consider the two-dimensional data $X$ shown in the top plot of Figure 5, consisting of three labeled classes (green, blue, red). Consider the four hyperplanes shown in the same plot, and suppose we had access only to the binary data $Q=$ $\operatorname{sign}(A X)$, where $A$ contains the normals to each hyperplane as its rows. For the new test point $X$ (which by visual inspection should be labeled blue) and its binary data $q=\operatorname{sign}(A x)$, one could simply cycle through the hyperplanes and decide which class $x$ matches most often. For example, for the hyperplane colored purple in the plot, $x$ has the same sign (i.e., lies on the same side) as the blue and green classes. For the black hyperplane, $x$ only matches the blue class, and so on. Then for this example, $x$ will clearly match the blue class most often, and we could assign it that label correctly. However, next consider the more complicated geometry given in the bottom plot, where the data consists of only two classes (red and blue), but they are now intermixed. This same strategy will no longer be accurate for the test point $x$. However, now instead of single hyperplanes, consider hyperplane pairs, and ask which class label $x$ most often matches (note that in this context, by "matches" we now mean that points lie in the same cone that the hyperplanes divide the space into). For example, for the pair colored orange and green, $x$ matches both red and blue points, whereas for the pair of hyperplanes colored orange and purple, $x$ only matches the blue class. One could now cycle through all pairs and again ask which class $x$ matches most often.

Let us now describe the approach more formally. Consider a data matrix $X \in \mathbb{R}^{d \times p}$. Let $A \in \mathbb{R}^{m \times d}$ have rows 

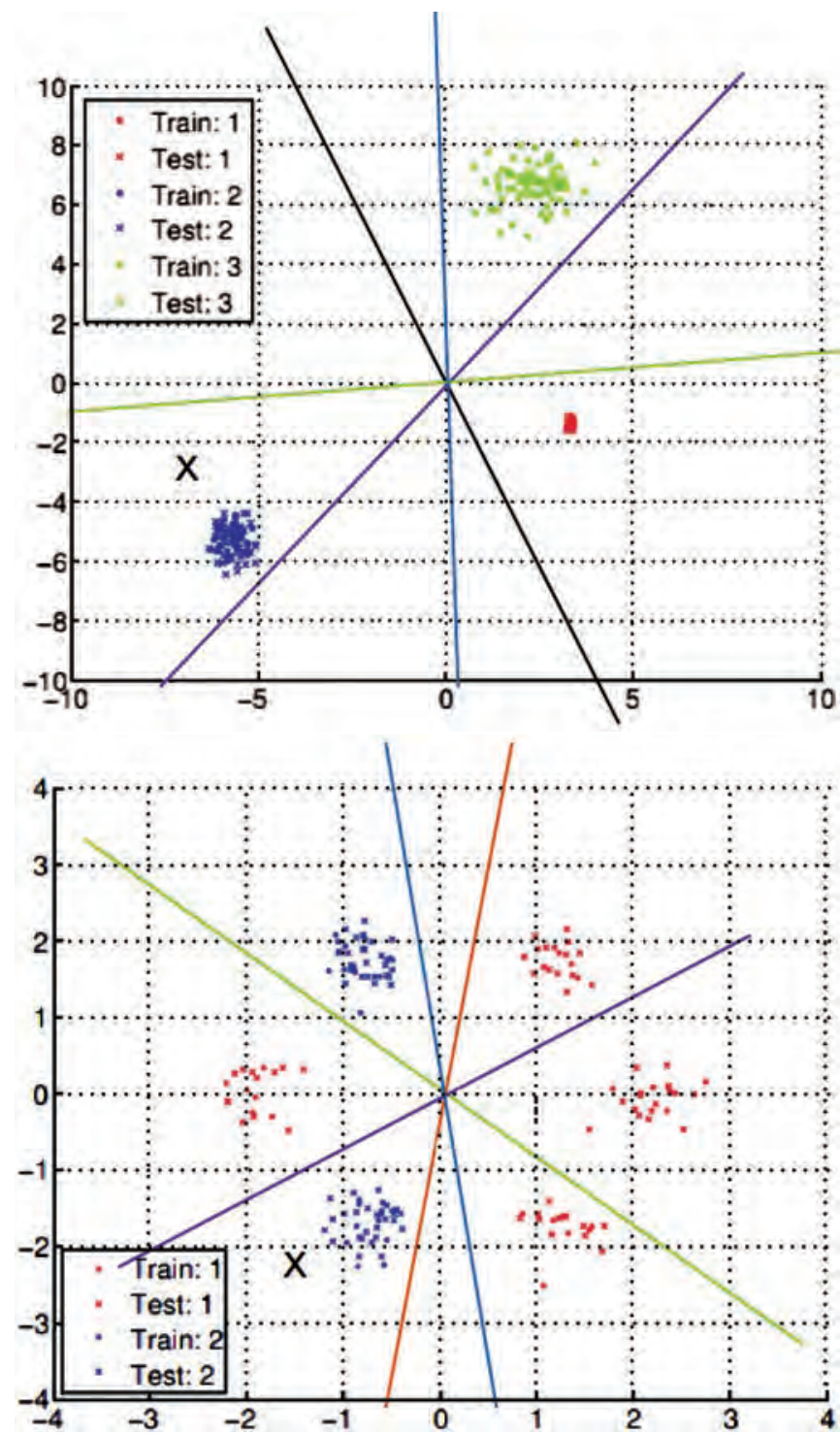

Figure 5. Figure 5. Two motivating examples for the classification method.

corresponding to the normal vectors of $m$ randomly oriented hyperplanes, and let $Q=\operatorname{sign}(A X)$ be the binary sign information. Then, the training algorithm proceeds in $L$ "levels." In the $\ell$ th level, $m$ index sets $\Lambda_{\ell, i} \subset[m]$, $\left|\Lambda_{\ell, i}\right|=\ell, i=1, \ldots, m$, are randomly selected. During the $i$ th "iteration" of the $\ell$ th level, the rows of $Q$ indexed by $\Lambda_{\ell, i}$ are used to form the $\ell \times p$ submatrix of $Q$, the columns of which define the sign patterns $\{ \pm 1\}^{\ell}$ observed by the training data. As in 1-bit CSP, these sign patterns contain the information about what sides of the hyperplanes the data points lie on.

At a given level $\ell$, for the $t$ th sign pattern (out of the possible $2^{\ell}$ ) and $g$ th class, a membership index parameter $r(\ell, i, t, g)$ that uses knowledge of the number of training points in class $g$ having the $t$ th sign pattern is calculated for every $\Lambda_{\ell, i}$. Larger values of $r(\ell, i, t, g)$ suggest that the $t$ th sign pattern is more heavily dominated by class $g$; thus, if a signal with an unknown label corresponds to the $t$ th sign pattern, we will be more likely to classify it into the $g$ th class. In [13], the authors define the following membership index parameter $r(\ell, i, t, g)$. Below, $P_{g \mid t}=P_{g \mid t}\left(\Lambda_{\ell, i}\right)$ denotes the number of training points from the $g$ th class with the $t$ th sign pattern at the $i$ th set selection in the $\ell$ th level:

$$
r(\ell, i, t, g)=\frac{P_{g \mid t}}{\sum_{j=1}^{G} P_{j \mid t}} \frac{\sum_{j=1}^{G}\left|P_{g \mid t}-P_{j \mid t}\right|}{\sum_{j=1}^{G} P_{j \mid t}} .
$$

Note that the first fraction in (4) indicates the proportion of training points in class $g$ out of all points with sign pattern $t$ (at the $\ell$ th level and $i$ th iteration). The second fraction in (4) is a balancing term that gives more weight to group $g$ when that group is much different in size than the others with the same sign pattern. Thus intuitively, these values should be large when the sign pattern $t$ is very popular among data points labeled as class $g$ and not as popular among other classes. With this intuition, we can then assign a label to a new test point $x$ using its binary data $q=\operatorname{sign}(A x)$. For each class $g$, we simply sum the membership index function values over all $\ell$ and $i$, for those sign patterns $t$ that match the sign pattern of the new test point $x$ (which is known via the data $q$ ). The label for $x$ is then decided by simply taking the class $g$ corresponding to the largest sum.

Figure 6 shows classification results for the Lyme data. For this result, we use the survey responses for the symptomrelated questions as our data matrix. This matrix consists of 3,686 "unwell" patients and 362 "well" patients (so 4, 048 patients in total) who each answered twelve symptomrelated questions (the "well" patients were asked about their worst symptoms while being sick). We randomly select a number (ten, fifty, or one hundred, see Figure 6) of those patients from each group to serve as our training data, and the remaining serve as our test data. We then run our method using this training and testing data and compute the correct classification rate, where the "ground truth" is determined from the separately asked question in the survey about whether the patient identifies as being "well" or "unwell." The top plot of Figure 6 demonstrates the ability to accurately identify well versus unwell patients from the symptoms (current or past) that they report. Since the "well" patients were asked about their worst prior symptoms, one might ask whether it is simply the case that "well" patients showcase higher (or lower) symptom levels in general, making classification easy. However, the bottom plot of the figure demonstrates this is not the case, and that perhaps more intricate and complex symptom patterns are at work. 


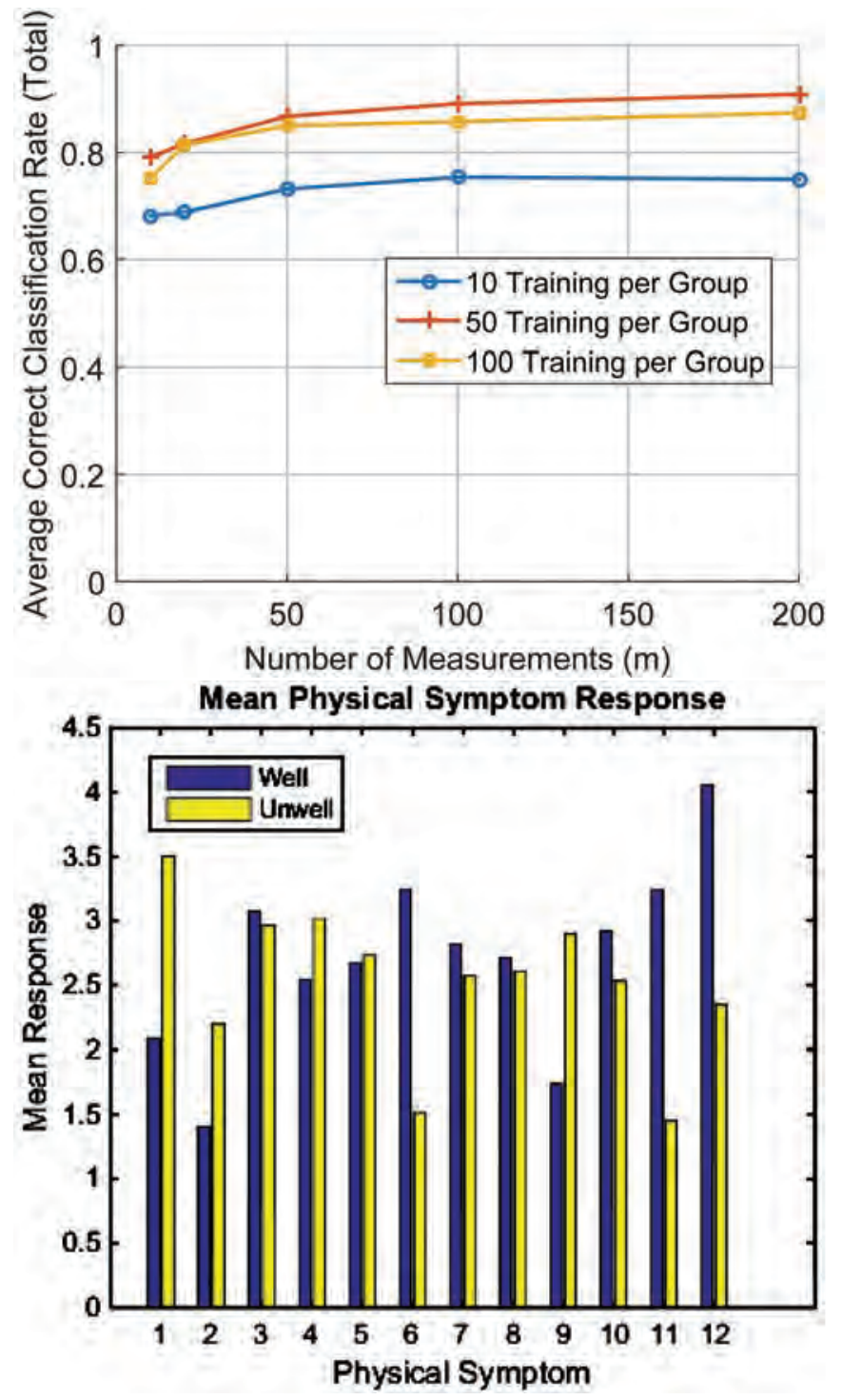

Figure 6. Figure 6. Top: Results from classification approach on symptom data using five layers for various numbers of randomly selected training points (patients). Bottom: Means on the survey questions for these groups.

\section{Conclusion}

Large-scale data is now ubiquitous, and the magnitude and abundance of data only continue to grow. Without proper mathematical techniques, this data will only bog down symptoms and stall scientific understanding. However, with sophisticated mathematical methods for large-scale data, the ability to acquire, store, complete, and analyze such data is possible. The recently acquired Lyme disease data is a perfect example of complicated data that has the ability to significantly enhance scientific and medical understanding. Unfortunately, clinical research of this illness has been somewhat stagnant, controversial, and challenging. That makes the use of big data and mathematical analysis even more critical to make progress toward better understanding, diagnosis, and treatment of Lyme disease and on other important scientific fronts.

\section{References}

[1] Baraniuk R, Foucart S, Needell D, Plan Y, Wootters M, Exponential decay of reconstruction error from binary measurements of sparse signals, IEEE Trans. Info. Theory, (63) no. 6: 3368-3385, 2017. MR3677739

[2] Boufounos PT, Baraniuk RG, 1-bit compressive sensing, 42nd Annual Conf. Info. Sciences and Systems (CISS), 16-21, 2008.

[3] Candès E, Tao T, The power of convex relaxation: Nearoptimal matrix completion, IEEE Trans. Inform. Theory, (56) no. 5: 2053-2080, 2010. MR2723472

[4] Candès EJ, Li X, Ma Y, Wright J, Robust principal component analysis?, Journal of the ACM, (58) no. 3: 1-37, 2011. MR2811000

[5] Candès EJ, Romberg J, Tao T, Stable signal recovery from incomplete and inaccurate measurements, Commun. Pur. Appl. Math., (59) no. 8: 1207-1223, 2006. MR2230846

[6] Candès EJ, Tao T, Decoding by linear programming, IEEE Trans. Info. Theory, (51): 4203-4215, 2005. MR2243152

[7] Eldar YC, Kutyniok G, Compressed sensing: theory and applications, Cambridge University Press, 2012. MR3254193

[8] Foucart S, Needell D, Plan Y, Wootters M, De-biasing lowrank projection for matrix completion, Proc. SPIE Optics and Photonics, 2017.

[9] Foucart S, Rauhut H, A mathematical introduction to compressive sensing, vol. 1, Birkhäuser Basel, 2013. MR3100033

[10] Knudson K, Saab R, Ward R, One-bit compressive sensing with norm estimation, IEEE Trans. Info. Theory, (62) no. 5: 2748-2758, 2016. MR3493876

[11] Lee T, Shraibman A, Matrix completion from any given set of observations, Adva. Neur. In., 1781-1787, 2013.

[12] Molitor D, Needell D, Matrix completion for structured observations, Proc. Information Theory and Approximation, 2018, to appear.

[13] Needell D, Saab R, Woolf T, Simple classification using binary data, 2017, submitted.

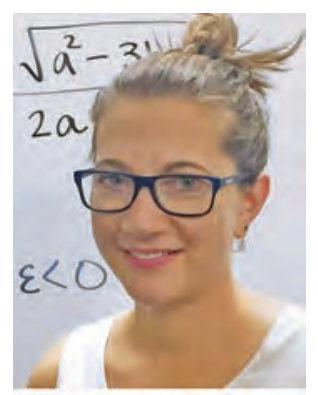

Deanna Needell 


\section{Credits}

Figure 1 is reprinted from Applied and Computational Harmonic Analysis, Volume 31, Issue 1, Emmanuel J. Candes, Yonina C. Eldar, Deanna Needell, and Paige Randall, Compressed Sensing with Coherent and Redundant Dictionaries, Pages 59-73, Copyright 2011, with permission from Elsevier.

Figure 2 is $\odot 2017$ IEEE. Reprinted, with permission, from Richard Baraniuk, Simon Foucart, Deanna Needell, Yaniv Plan, and Mary Wootters. Exponential decay of reconstruction error from binary measurements of sparse signals. IEEE Transactions on Information Theory. Volume: 63, Issue: 6, June 2017.

Figures 3-6 are courtesy of the author. Author photo is by Blake Hunter.

\section{Advertise in the

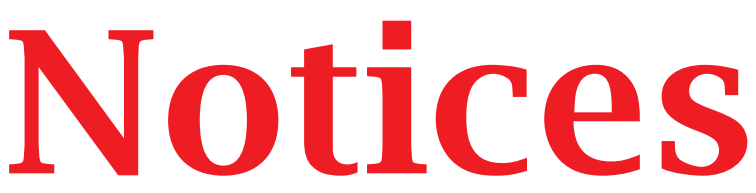

of the American Mathematical Society

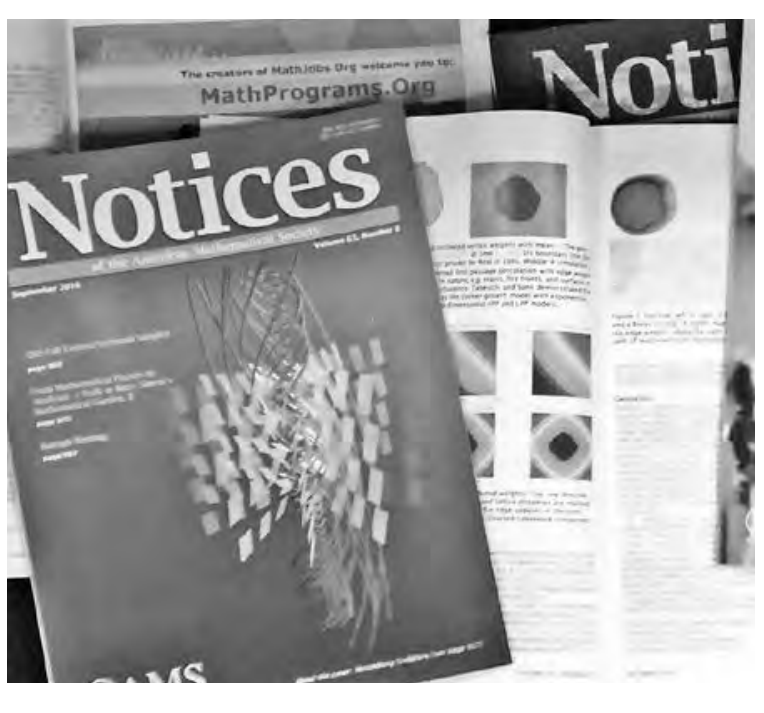

Connect with an audience of approximately 30,000 subscribers through Classified and Display Advertising in the Notices of the American Mathematical Society, available in print and online.

As the world's most widely read magazine aimed at professional mathematicians, the Notices is an excellent medium for announcing publications, products, and services, and for recruiting mathematical scientists in academic, industry, and government.

Learn more about advertising in the Notices at

www.ams.org/noticesadvertising.

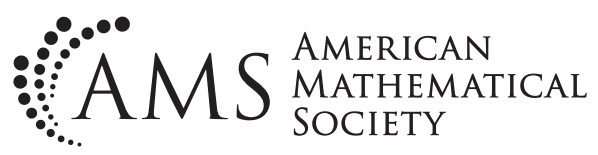




\section{Iwasawa Theory: A Climb up the Tower}

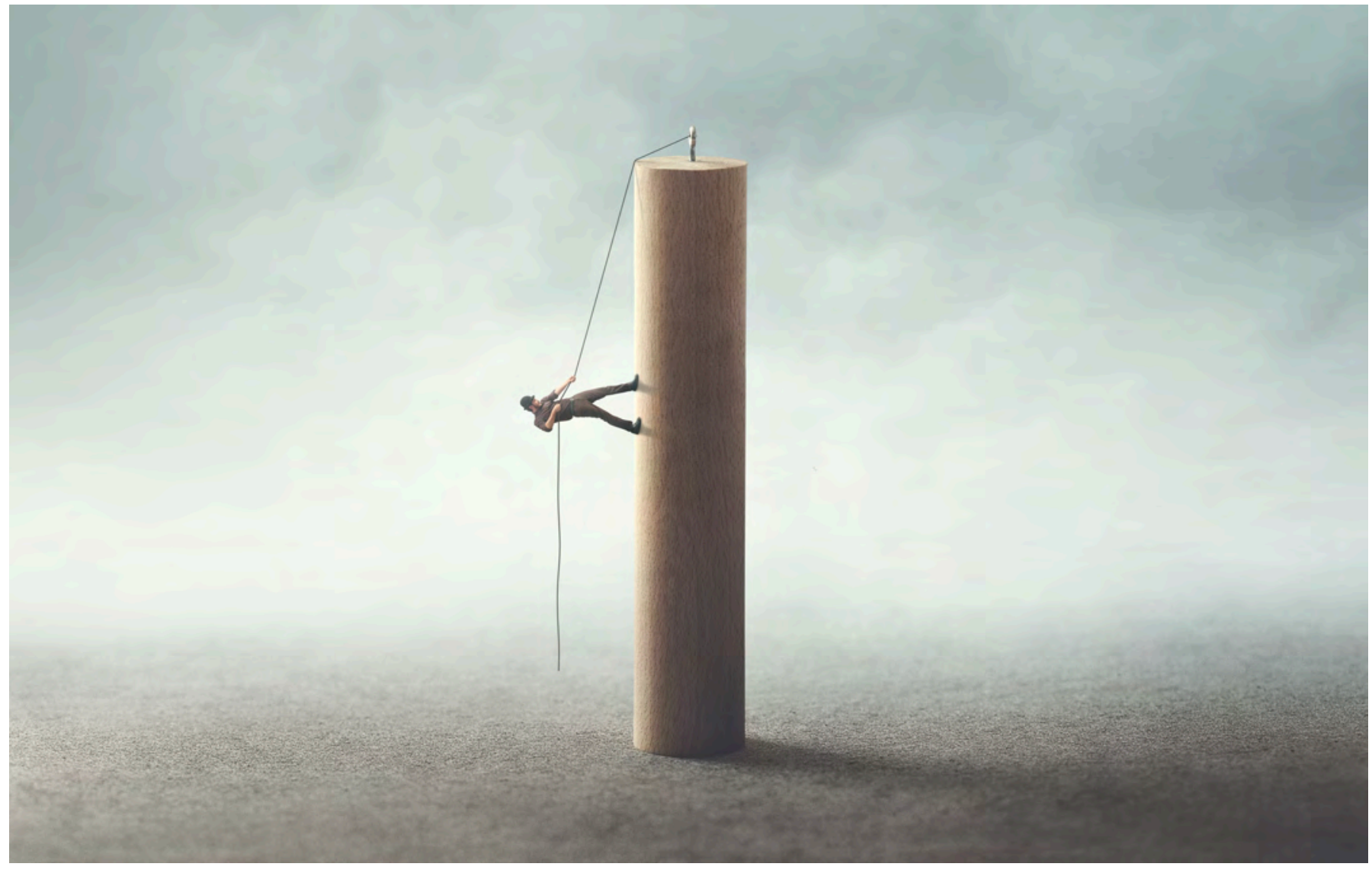

\section{Romyar Sharifi}

Iwasawa theory is an area of number theory that emerged from the foundational work of Kenkichi Iwasawa in the late 1950s and onward. It studies the growth of arithmetic objects, such as class groups, in towers of number fields. Its key observation is that a part of this growth exhibits a remarkable regularity, which it aims to describe in terms of values of meromorphic functions known as $L$-functions, such as the Riemann zeta function.

Romyar Sharifi is a professor of mathematics at UCLA. His email address is sharifi@math.ucla.edu.

Communicated by Notices Associate Editor Daniel Krashen.

This material is based upon work supported by the National Science Foundation under Grant Nos. 1661658 and 1801963.

For permission to reprint this article, please contact:

reprint-permission@ams.org.

DOI: https://doi.org/10.1090/noti/1759
Through such descriptions, Iwasawa theory unveils intricate links between algebraic, geometric, and analytic objects of an arithmetic nature. The existence of such links is a common theme in many central areas within arithmetic geometry. So it is that Iwasawa theory has found itself a subject of continued great interest. This year's Arizona Winter School attracted nearly 300 students hoping to learn about it!

The literature on Iwasawa theory is vast and often technical, but the underlying ideas possess undeniable beauty. I hope to convey some of this while explaining the original questions of Iwasawa theory and giving a sense of the directions in which the area is heading. 


\section{Algebraic Number Theory}

To understand Iwasawa theory requires some knowledge of the background out of which it arose. We attempt to chart a course, beginning with a whirlwind tour of the elements of algebraic number theory. We make particular note of two algebraic objects, the class group and the unit group of a number field, that play central roles for us.

Algebraic numbers are the roots inside the complex numbers of nonzero polynomials in a single variable with rational coefficients. They lie in finite field extensions of $\mathbb{Q}$ called number fields. The set of algebraic numbers forms a subfield $\overline{\mathbb{Q}}$ of $\mathbb{C}$ known as an algebraic closure of $\mathbb{Q}$. Inside $\overline{\mathbb{Q}}$ sits a subring $\overline{\mathbb{Z}}$ of algebraic integers, consisting of the roots of monic polynomials with integer coefficients.

The field automorphisms of $\overline{\mathbb{Q}}$ form a huge group called the absolute Galois group $G_{\mathbb{Q}}=\operatorname{Gal}(\overline{\mathbb{Q}} / \mathbb{Q})$. These automorphisms permute the roots of each rational polynomial, and consequently this action preserves the algebraic integers.

We'll use $F$ to denote a number field. The integer ring $\mathcal{O}_{F}$ of $F$ is the subring of algebraic integers in $F$. It is a PID if and only if it's a UFD, but unlike $\mathcal{O}_{\mathbb{Q}}=\mathbb{Z}$, it need not in general be either. Rather, $\mathcal{O}_{F}$ is what is known as a Dedekind domain. As such, it has the property that every nonzero ideal factors uniquely up to ordering into a product of prime ideals. This property provides a replacement for unique factorization of elements. A "prime" of $F$ is a nonzero prime ideal of $\mathcal{O}_{F}$.

A fractional ideal of $F$ is a nonzero, finitely generated $\mathcal{O}_{F}$-submodule of $F$. Nonzero ideals of $\mathcal{O}_{F}$ are fractional ideals, but so are, for instance, all $\mathcal{O}_{F}$-multiples of $\frac{1}{2}$. The set $I_{F}$ of fractional ideals is an abelian group under multiplication with identity $\mathcal{O}_{F}$. The class group $\mathrm{Cl}_{F}$ of $F$ is the quotient of $I_{F}$ by its subgroup of principal fractional ideals generated by nonzero elements of $F$. The group $\mathrm{Cl}_{F}$ is trivial if and only if $\mathcal{O}_{F}$ is a PID.

Remarkably, $\mathrm{Cl}_{F}$ is finite. Its order $h_{F}$ is known as the class number. Like the class group itself, it is the subject of many open questions. For instance, work of Heegner, Baker, and Stark in the 1950s and '60s solved a problem of Gauss by showing that there are exactly nine imaginary quadratic fields with class number one: $\mathbb{Q}(i), \mathbb{Q}(\sqrt{-2})$, $\mathbb{Q}(\sqrt{-3}), \mathbb{Q}(\sqrt{-7}), \ldots$. On the other hand, Gauss' conjecture that there are infinitely many such real quadratic fields is still open.

A number field $F$ can be viewed as a subfield of $\mathbb{C}$ in multiple ways. That is, any $\sigma \in G_{\mathbb{Q}}$ gives an isomorphism $\sigma: F \rightarrow \sigma(F)$, and $\sigma(F)$ is a subfield of $\mathbb{C}$ as well, so precomposition with $\sigma$ yields a different "archimedean" embedding of $F$ in $\mathbb{C}$. We may then place a metric on $F$ by restricting the usual distance function. An embedding of $F$ in $\mathbb{C}$ is real if it has image in $\mathbb{R}$ and complex if it is not real, in which case it has dense image in $\mathbb{C}$. The numbers of real and complex-conjugate pairs of complex embeddings are respectively denoted $r_{1}(F)$ and $r_{2}(F)$.

The unit group $\mathcal{O}_{F}^{\times}$of invertible elements in $\mathcal{O}_{F}$ under multiplication is deeply intertwined with the class group $\mathrm{Cl}_{F}$. In fact, these groups are the kernel and cokernel of the map $F^{\times} \rightarrow I_{F}$ taking an element to its principal fractional ideal. Dirichlet's unit theorem says that $\mathcal{O}_{F}^{\times}$is a direct product of the group of roots of unity in $F$ and a free abelian group of rank $r=r_{1}(F)+r_{2}(F)-1$. This is proven using logarithms of absolute values of units with respect to archimedean embeddings. The regulator $R_{F}$ of $F$ is a nonzero real number defined as a determinant of a matrix formed out of such logarithms.

The prototypical example is $F=\mathbb{Q}(\sqrt{-5})$, for which $\mathcal{O}_{F}=\mathbb{Z}[\sqrt{-5}]$. One has $r_{1}(F)=0, r_{2}(F)=1$, and $\mathcal{O}_{F}^{\times}=\{ \pm 1\}$. We have two factorizations of 6 into irreducible elements of $\mathbb{Z}[\sqrt{-5}]$ that don't differ up to units:

$$
6=2 \cdot 3=(1+\sqrt{-5})(1-\sqrt{-5}) .
$$

The unique factorization into primes that resolves this for our purposes is

$$
\text { (6) }=(2,1+\sqrt{-5})^{2}(3,1+\sqrt{-5})(3,1-\sqrt{-5}) \text {. }
$$

The class number of $\mathbb{Q}(\sqrt{-5})$ is 2 , so the class group is generated by the class of any nonprincipal ideal, such as $(2,1+\sqrt{-5})$ or $(3,1 \pm \sqrt{-5})$.

Ramification in an extension of number fields is akin to the phenomenon of branching in branched covers in topology. As we've implicitly noted, the factorization of (2) in $\mathbb{Z}[\sqrt{-5}]$ is $(2,1+\sqrt{-5})^{2}$. The square is telling us that the same prime is occurring (at least) twice in the factorization: this is the branching. Whenever this happens in an extension of number fields, we say that the prime of the base field ramifies in the extension. Only finitely many primes ramify in an extension of number fields.

The Dedekind zeta function of $F$ is the unique meromorphic continuation to $\mathbb{C}$ of the series

$$
\zeta_{F}(s)=\sum_{\mathfrak{a} \subset \mathcal{O}_{F}}(N \mathfrak{a})^{-s},
$$

where $\mathfrak{a}$ runs over the nonzero ideals of $\mathcal{O}_{F}$ and $N \mathfrak{a}$ is the index of $\mathfrak{a}$ in $\mathcal{O}_{F}$. It has a simple pole at $s=1$ and satisfies a functional equation relating $\zeta_{F}(s)$ and $\zeta_{F}(1-s)$ that involves factors coming from the archimedean embeddings. The Dedekind zeta function of $\mathbb{Q}$ is the ubiquitous Riemann zeta function $\zeta(s)$.

The functional equation tells us that $\zeta_{F}(s)$ vanishes to order the rank $r$ of $\mathcal{O}_{F}^{\times}$at $s=0$. The leading term in its Taylor expansion is given by the analytic class number formula

$$
\frac{\zeta_{F}^{(r)}(0)}{r !}=-\frac{h_{F} R_{F}}{w_{F}}
$$


where $w_{F}$ is the number of roots of unity in $F$. This provides an important instance of a meromorphic function intertwining the unit and class groups. More broadly, it's a first fundamental example of the links between analytic and algebraic objects of arithmetic.

To a prime $\mathfrak{p}$ of $F$, we can attach a $\mathfrak{p}$-adic metric under which elements are closer together if their difference lies in a higher power of $\mathfrak{p}$. We may complete $F$ with respect to this metric to obtain a complete "local" field $F_{\mathfrak{p}}$ that has a markedly non-Euclidean topology. It has a compact valuation ring $\mathcal{O}_{\mathfrak{p}}$ equal to the open and closed unit ball about 0 .

For example, the $p$-adic metric $d_{p}$ on $\mathbb{Q}$ is defined by $d_{p}(x, y)=p^{-n}$ for the largest $n \in \mathbb{Z}$ such that $x-y \in$ $p^{n} \mathbb{Z}$. The completion of $\mathbb{Q}$ with respect to $d_{p}$ is called the $p$-adic numbers $\mathbb{Q}_{p}$, which has valuation ring the $p$-adic integers $\mathbb{Z}_{p}$. Alternatively, $\mathbb{Z}_{p}$ is the inverse limit of the rings $\mathbb{Z} / p^{n} \mathbb{Z}$ under the reduction maps between them.

Every prime $\mathfrak{p}$ of $F$ is maximal in $\mathcal{O}_{F}$ with finite residue field $\mathcal{O}_{F} / \mathfrak{p}$. The Galois group $G$ of a finite Galois extension $E / F$ acts transitively on the set of primes $\mathfrak{q}$ of $E$ containing $\mathfrak{p}$. The stabilizer of $\mathfrak{q}$ in $G$ is called its decomposition group $G_{\mathfrak{q}}$ and is isomorphic to $\operatorname{Gal}\left(E_{\mathfrak{q}} / F_{\mathfrak{p}}\right)$. The extension $\mathcal{O}_{E} / \mathfrak{q}$ of $\mathcal{O}_{F} / \mathfrak{p}$ has cyclic Galois group generated by $\chi \mapsto \chi^{N \mathfrak{p}}$. This element lifts to an element of $G_{\mathfrak{q}}$ called a Frobenius element at $\mathfrak{q}$. The lift is unique if $\mathfrak{p}$ is unramified in $E / F$. It is independent of $\mathfrak{q}$ if $G$ is also abelian, in which case we denote it by $\varphi_{\mathfrak{p}}$.

The Hilbert class field $H_{F}$ of $F$ is the largest unramified abelian extension of $F$. Here, "unramified" means that no prime ramifies and no real embedding becomes complex. The Artin map taking the class of a prime $\mathfrak{p}$ to $\varphi_{\mathfrak{p}}$ provides an isomorphism

$$
\mathrm{Cl}_{F} \stackrel{\sim}{\longrightarrow} \mathrm{Gal}\left(H_{F} / F\right) .
$$

For example, the Hilbert class field of $\mathbb{Q}(\sqrt{-5})$ is $\mathbb{Q}(\sqrt{5}, i)$, and the Artin isomorphism tells us whether or not a prime $\mathfrak{p}$ of $\mathbb{Z}[\sqrt{-5}]$ is principal via the sign in $\varphi_{\mathfrak{p}}(i)$ $= \pm i$. Class field theory concerns "reciprocity maps" generalizing the Artin isomorphism by relaxing the ramification conditions. These can be used to prove reciprocity laws generalizing Gauss' law of quadratic reciprocity.

\section{Cyclotomic Fields}

Iwasawa theory has its origins in the study of the arithmetic of cyclotomic fields, a classical area of number theory that dates back to attempts at proving Fermat's last theorem in the mid-1800s. This is a fascinating subject in its own right, not least for the connections it reveals between Bernoulli numbers and the structure of cyclotomic class groups.

For a positive integer $N$, the $N$ th cyclotomic field $\mathbb{Q}\left(\zeta_{N}\right)$ is given by adjoining the primitive $N$ th root of unity $\zeta_{N}=$ $e^{2 \pi i / N}$ to $\mathbb{Q}$. The Kronecker-Weber theorem states that every finite abelian extension of the rationals is contained in a cyclotomic field. If $N>1$, the prime ideals that ramify in $\mathbb{Q}\left(\zeta_{2 N}\right)$ are exactly those dividing $N$. The integer ring of $\mathbb{Q}\left(\zeta_{N}\right)$ is $\mathbb{Z}\left[\zeta_{N}\right]$, so every cyclotomic integer is a $\mathbb{Z}$-linear combination of roots of unity.

Each element $\sigma$ of $\operatorname{Gal}\left(\mathbb{Q}\left(\zeta_{N}\right) / \mathbb{Q}\right)$ carries $\zeta_{N}$ to another primitive $N$ th root of unity, which has the form $\zeta_{N}^{i}$ for some $i$ prime to $N$. The map taking $\sigma$ to the unit $i$ modulo $N$ provides an isomorphism

$$
\operatorname{Gal}\left(\mathbb{Q}\left(\zeta_{N}\right) / \mathbb{Q}\right) \stackrel{\sim}{\longrightarrow}(\mathbb{Z} / N \mathbb{Z})^{\times}
$$

known as the $N$ th cyclotomic character.

For $x, y$, and $z$ satisfying the Fermat equation in odd prime exponent $p$, we have a factorization

$$
x^{p}+y^{p}=\prod_{i=0}^{p-1}\left(x+\zeta_{p}^{i} y\right)=z^{p}
$$

in $\mathbb{Z}\left[\zeta_{p}\right]$. Using this, Kummer proved in an 1850 paper

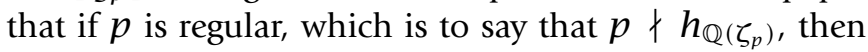
Fermat's last theorem holds in exponent $p$. (Its use lies in the fact that if $p$ is regular, then $\left(x+\zeta_{p}^{i} y\right)$ cannot be the $p$ th power of a nonprincipal ideal.) It's known that there are infinitely many irregular primes but not that there are infinitely many regular primes, though over sixty percent of primes up to any given number are expected to be regular.

For $k \geq 0$, the $k$ th Bernoulli number $B_{k} \in \mathbb{Q}$ is the $k$ th derivative at 0 of the function $\frac{x}{e^{x}-1}$. One has $B_{k}=0$ for odd $k \geq 3$. Here's a table of Bernoulli numbers for positive even indices:

\begin{tabular}{|c|c|c|c|c|c|c|c|}
\hline$k$ & 2 & 4 & 6 & 8 & 10 & 12 & 14 \\
\hline$B_{k}$ & $\frac{1}{6}$ & $-\frac{1}{30}$ & $\frac{1}{42}$ & $-\frac{1}{30}$ & $\frac{5}{66}$ & $-\frac{691}{2730}$ & $\frac{7}{6}$ \\
\hline
\end{tabular}

Kummer proved the following, which amounts to a special case of the class number formula.

Theorem (Kummer). A prime $p$ is irregular if and only if $p$ divides the numerator of $B_{k}$ for some positive even $k<p$.

In particular, 691 is irregular as it divides $B_{12}$. Here's a table of irregular primes $p<150$ and the indices $n$ of the Bernoulli numbers $B_{n}$ they divide:

\begin{tabular}{|c|c|c|c|c|c|c|c|}
\hline$p$ & 37 & 59 & 67 & 101 & 103 & 131 & 149 \\
\hline$n$ & 32 & 44 & 58 & 68 & 24 & 22 & 130 \\
\hline
\end{tabular}

The prime 157 divides both $B_{62}$ and $B_{110}$. The index of irregularity $i_{p}$ of $p$ is the number of Bernoulli numbers $B_{k}$ with $k<p$ even that $p$ divides. Its values up to an increasingly large bound are expected to fit a Poisson distribution with parameter $\frac{1}{2}$. The latest in a long history of computations is due to Hart, Harvey, and Ong for $p<2^{31}=$ 2147483648 . In this range, $i_{p}$ attains a maximum value of 9 . 
Regularity of $p$ is equivalent to the triviality of the Sylow $p$-subgroup $A$ of $\mathrm{Cl}_{\mathbb{Q}\left(\zeta_{p}\right)}$. We call $A$ the $p$-part of the class group. So, what more does the fact that an odd $p$ divides a particular Bernoulli number tell us about $A$ ? The answer is found in the action of

$$
\Delta=\operatorname{Gal}\left(\mathbb{Q}\left(\zeta_{p}\right) / \mathbb{Q}\right) \cong(\mathbb{Z} / p \mathbb{Z})^{\times}
$$

on $A$ induced by the $\Delta$-action on field elements.

For each $\delta \in(\mathbb{Z} / p \mathbb{Z})^{\times}$, there is a unique element $\omega(\delta)$ $\in \mathbb{Z}_{p}^{\times}$of order dividing $p-1$ that reduces to $\delta$. The resulting homomorphism

$$
\omega:(\mathbb{Z} / p \mathbb{Z})^{\times} \rightarrow \mathbb{Z}_{p}^{\times}
$$

is a splitting of the reduction modulo $p$ map. The group $A$ breaks up as a direct sum

$$
A=\bigoplus_{i \in \mathbb{Z} /(p-1) \mathbb{Z}} A^{(i)}
$$

of subgroups

$$
A^{(i)}=\left\{a \in A \mid \delta(a)=\omega(\delta)^{i} a \text { for all } \delta \in \Delta\right\}
$$

for integers $i$ modulo $p-1$. For obvious reasons, these are often called $\Delta$-eigenspaces of $A$. We shall seek to study such eigenspaces, beginning with the following important theorem.

Theorem (Herbrand-Ribet). If $k$ is a positive even integer with $k<p$, then $p$ divides $B_{k}$ if and only if $A^{(p-k)} \neq 0$.

Herbrand proved in 1932 that $A^{(p-k)}=0$ unless $p$ divides $B_{k}$. Ribet proved the converse in a 1976 paper [Ri]. The proof of Herbrand's theorem relies on a direct construction of an annihilator of the class group in $\mathbb{Z}[\Delta]$ due to Stickelberger. Ribet's proof is more delicate, involving a congruence between modular forms that occurs when $p$ divides $B_{k}$ and its consequences for a Galois representation. We'll explain his method later.

There is also a coarser decomposition of $A$ as $A^{+} \oplus A^{-}$, where $A^{ \pm}$is the subgroup of elements on which complex conjugation acts as \pm 1 . Then $A^{ \pm}$is the direct sum of the $\Delta$ eigenspaces of $A$ for even/odd $i$. The Herbrand-Ribet theorem concerns $A^{-}$, but Kummer had already shown that $A^{-}=0$ implies $A=0$.

The order of $A^{+}$is the highest power of $p$ dividing the class number $h^{+}$of the fixed field $\mathbb{Q}\left(\zeta_{p}\right)^{+}$of complex conjugation. In 1920, Vandiver rediscovered and later popularized a conjecture of Kummer's that $A^{+}=0$. Hart, Harvey, and Ong have verified this conjecture for $p<2^{31}$.

Vandiver's conjecture can be rephrased as a question about units. That is, the group of cyclotomic units in $\mathbb{Z}\left[\zeta_{p}\right]$ is generated by

$$
1+\zeta_{p}+\cdots+\zeta_{p}^{j-1} \text { for } 1<j<p .
$$

The class number formula implies that the index of this subgroup of the unit group $\mathbb{Z}\left[\zeta_{p}\right]^{\times}$is $h^{+}$. Vandiver's conjecture asserts that $p$ does not divide this index.
By a reflection principle of Leopoldt, an even eigenspace $A^{(k)}$ vanishes if $A^{(p-k)}=0$, while $A^{(k)}=0$ implies that $A^{(p-k)}$ is cyclic. The proof of this uses class field theory and a general duality between Galois and field elements known as Kummer theory.

\section{Classical Iwasawa Theory}

Iwasawa theory concerns the growth of arithmetic objects in towers of number fields. More precisely, it concerns the growth of $p$-parts of class groups, and more general objects called Selmer groups, in towers of number fields of $p$-power degree. This growth exhibits a certain regularity that in good circumstances can be partially described by a $p$-adic variant of a complex-valued $L$-function.

The simplest sort of tower consists of a sequence $F_{n}$ of fields

$$
F=F_{0} \subset F_{1} \subset F_{2} \subset \cdots \subset F_{\infty}=\bigcup_{n=0}^{\infty} F_{n}
$$

with $F_{n} / F$ cyclic of degree $p^{n}$. The Galois group $\Gamma=$ $\operatorname{Gal}\left(F_{\infty} / F\right)$ of the tower is the inverse limit of the groups $\Gamma_{n}=\operatorname{Gal}\left(F_{n} / F\right) \cong \mathbb{Z} / p^{n} \mathbb{Z}$ and as such is isomorphic to the additive group of $\mathbb{Z}_{p}$. It is thus a compact group under the $p$-adic topology. Every number field $F$ has a cyclotomic $\mathbb{Z}_{p}$-extension defined as the unique $\mathbb{Z}_{p}$-extension of $F$ inside the union of the fields $F\left(\zeta_{p^{n}}\right)$.

If $F$ has more than one $\mathbb{Z}_{p}$-extension, it has infinitely many. Yet, the Galois group of the compositum of all $\mathbb{Z}_{p}$ extensions still has finite $\mathbb{Z}_{p}$-rank $t \geq r_{2}(F)+1$. Leopoldt conjectured this to be an equality in 1962. This notoriously difficult conjecture can be phrased as the equality of the $\mathbb{Z}$-rank of the unit group $\mathcal{O}_{F}^{\times}$and the $\mathbb{Z}_{p}$-rank of the closure of its image inside the direct sum of its local completions at primes over $p$. Leopoldt's conjecture is known for abelian extensions of $\mathbb{Q}$ and imaginary quadratic fields by 1967 work of Brumer.

Let's fix a $\mathbb{Z}_{p}$-extension $F_{\infty}$ of $F$ in our discussion. In 1959 , Iwasawa proved a result on the growth of the orders of the $p$-parts $A_{n}$ of the class groups of the fields $F_{n}$ [Iw1].

Theorem (Iwasawa). There exist nonnegative integers $\lambda$ and $\mu$ and an integer $v$ such that

$$
\left|A_{n}\right|=p^{p^{n} \mu+n \lambda+v}
$$

for all sufficiently large $n$.

As a $p$-group, $A_{n}$ is a module over $\mathbb{Z}_{p}$. Since it also has a commuting $\Gamma_{n}$-action, $A_{n}$ is a module for the group ring $\mathbb{Z}_{p}\left[\Gamma_{n}\right]$, which consists of finite formal sums of elements of $\Gamma_{n}$ with $\mathbb{Z}_{p}$-coefficients.

We can compare the $A_{n}$ via norm maps $A_{n+1} \rightarrow A_{n}$ for every $n \geq 0$, as well as via maps $A_{n} \rightarrow A_{n+1}$ induced by the inclusion of $F_{n}$ in $F_{n+1}$. These maps are compatible with the action of $\mathbb{Z}_{p}\left[\Gamma_{n+1}\right]$ on both sides, with the action 
on $A_{n}$ arising through the restriction map $\Gamma_{n+1} \rightarrow \Gamma_{n}$. The Iwasawa algebra

$$
\Lambda=\lim _{n} \mathbb{Z}_{p}\left[\Gamma_{n}\right]
$$

then acts on both the inverse $\operatorname{limit} \lim _{n} A_{n}$ and the direct limit $A_{\infty}=\lim _{n} A_{n} A_{n}$. The Iwasawa algebra is a completion of the usual group ring $\mathbb{Z}_{p}[\Gamma]$, and as such it is a compact topological ring. Modules over $\Lambda$ are also known as Iwasawa modules.

The Artin isomorphism identifies $A_{n}$ with the Galois group $\operatorname{Gal}\left(L_{n} / F_{n}\right)$ of the maximal unramified abelian $p$ extension (i.e., of $p$-power degree) $L_{n}$ of $F_{n}$. These maps are compatible with norms on class groups and restriction on Galois groups.

The inverse limit of Artin isomorphisms identifies $\lim _{n} A_{n}$ with the Galois group

$$
X_{\infty}=\operatorname{Gal}\left(L_{\infty} / F_{\infty}\right),
$$

where $L_{\infty}=\bigcup_{n} L_{n}$. As an inverse limit of finite $p$-groups, $X_{\infty}$ is said to be pro- $p$, and $L_{\infty}$ is the maximal unramified abelian pro- $p$-extension of $F_{\infty}$.

The $\Gamma$-action on the inverse limit of the $A_{n}$ is identified via the Artin isomorphisms with the conjugation action of $\Gamma$ on $X_{\infty}$. For this, one lifts $\gamma \in \Gamma$ to $\tilde{\gamma} \in \operatorname{Gal}\left(L_{\infty} / F\right)$ and allows $\gamma$ to act on $\sigma \in X_{\infty}$ by

$$
\gamma: \sigma \mapsto \tilde{\gamma} \sigma \tilde{\gamma}^{-1} .
$$

This gives $X_{\infty}$ the structure of a $\Lambda$-module, and we refer to $X_{\infty}$ as the unramified Iwasawa module. In that each $A_{n}$ is finite, $X_{\infty}$ is finitely generated and torsion over $\Lambda$.

The $\Lambda$-module $X_{\infty}$ is compact, while $A_{\infty}$ is discrete. To obtain a compact $\Lambda$-module from $A_{\infty}$, we take its Pontryagin dual $A_{\infty}^{\vee}=\operatorname{Hom}\left(A_{\infty}, \mathbb{Q}_{p} / \mathbb{Z}_{p}\right)$, which is again finitely generated and torsion. Its structure is very closely related to that of $X_{\infty}$.

In good circumstances, such as when $F_{\infty}$ is the cyclotomic $\mathbb{Z}_{p}$-extension of $F=\mathbb{Q}\left(\zeta_{p}\right)$, we can recover $A_{n}$ from $X_{\infty}$ as the largest quotient of $X_{\infty}$ upon which $\Gamma^{p^{n}}$ acts trivially. Crucial to this is the fact that $\Gamma$ is a pro- $p$ group. Because of this, $\Lambda$ is a local ring, and one can employ Nakayama's lemma. This stands in stark contrast to the case of finite Galois extensions of prime-to- $p$ degree, for which one has far less control over the growth of $p$ parts of class groups. Nevertheless, Washington showed in 1979 that the $p$-parts eventually stop growing in the cyclotomic $\mathbb{Z}_{\ell}$-extension for $\ell \neq p$ of an abelian extension of $\mathbb{Q}$.

As observed by Serre, the Iwasawa algebra $\Lambda$ is isomorphic to a power series ring $\mathbb{Z}_{p} \llbracket T \rrbracket$ in a single variable $T$. For this, we fix a topological generator $\gamma$ of $\Gamma$, which is to say an element generating a dense subgroup or, equivalently, an element that restricts to a generator of each $\Gamma_{n}$.
There is then a unique continuous isomorphism of compact $\mathbb{Z}_{p}$-algebras that takes $\gamma-1$ to $T$. We shall use such an isomorphism to identify $\Lambda$ and $\mathbb{Z}_{p} \llbracket T \rrbracket$.

The structure theory of finitely generated modules over $\Lambda$ mimics the theory of finitely generated modules over a PID if one treats $\Lambda$-modules as being defined up to finite submodules and quotient modules. The idea is that $\Lambda$ becomes a PID upon localization at any principal prime ideal, and there are no nonzero finite modules over the localization.

A homomorphism $f: M \rightarrow N$ of finitely generated $\Lambda$ modules is called a pseudo-isomorphism if it has finite kernel and cokernel. The notion of pseudo-isomorphism gives an equivalence relation on any set of finitely generated, torsion $\Lambda$-modules.

Theorem (Iwasawa, Serre). For any finitely generated, torsion $\Lambda$-module $M$, there is a pseudo-isomorphism

$$
M \rightarrow \bigoplus_{i=1}^{r} \Lambda /\left(f_{i}^{k_{i}}\right) \oplus \bigoplus_{j=1}^{s} \Lambda /\left(p^{m_{j}}\right),
$$

where $r, s \geq 0$, each $f_{i}$ is a monic irreducible polynomial in $\mathbb{Z}_{p}[T]$ satisfying $f_{i} \equiv T^{\operatorname{deg} f_{i}} \bmod p$, and each $k_{i}$ and $m_{j}$ is a positive integer.

In the notation of the theorem, we set

$$
\lambda(M)=\sum_{i=1}^{r} k_{i} \operatorname{deg} f_{i} \text { and } \mu(M)=\sum_{j=1}^{s} m_{j} .
$$

We can also associate to $M$ its characteristic ideal $\operatorname{char}(M)$ in $\Lambda$. That is, given the above pseudo-isomorphism, we define

$$
\operatorname{char}(M)=\left(p^{\mu(M)} \prod_{i=1}^{r} f_{i}^{k_{i}}\right) .
$$

The polynomial $\prod_{i=1}^{r} f_{i}^{k_{i}}$ is the usual characteristic polynomial of $T$ acting on the finite-dimensional $\mathbb{Q}_{p}$-vector space $M \otimes_{\mathbb{Z}_{p}} \mathbb{Q}_{p}$.

In the case of the unramified Iwasawa module $X_{\infty}$, the quantities $\lambda=\lambda\left(X_{\infty}\right)$ and $\mu=\mu\left(X_{\infty}\right)$ are those in Iwasawa's growth formula. In fact, we also have $\lambda=\lambda\left(A_{\infty}^{\vee}\right)$ and $\mu=\mu\left(A_{\infty}^{\vee}\right)$, with the characteristic ideals of $X_{\infty}$ and $A_{\infty}^{\vee}$ differing by the change of variables $T \mapsto(1+T)^{-1}-1$.

Iwasawa conjectured that $\mu=0$ if $F_{\infty}$ is the cyclotomic $\mathbb{Z}_{p}$-extension of $F$. Ferrero and Washington proved that this holds when $F / \mathbb{Q}$ is abelian [FW]. In this case, Iwasawa also showed that the $(-1)$-eigenspace $X_{\infty}^{-}$for the action of complex conjugation has no finite $\Lambda$-submodule.

For $F_{\infty}$ the cyclotomic $\mathbb{Z}_{p}$-extension of $F=\mathbb{Q}\left(\mu_{p}\right)$, the Iwasawa module $X_{\infty}$ has an action of $\Delta=\operatorname{Gal}(F / \mathbb{Q})$ that commutes with its $\Lambda$-action, so we can again break up $X_{\infty}$ as a direct sum of $\Delta$-eigenspaces. In the computations of Hart-Harvey-Ong, not a single $\Delta$-eigenspace of $X_{\infty}$ has $\lambda$-invariant greater than 1 . Such an eigenspace 
$X_{\infty}^{(i)}$ is nonzero if and only if $A^{(i)}$ is nonzero, so these computations imply that $\lambda \leq 9$ for $p<2^{31}$.

\section{$p$-adic $L$-Functions}

For any positive integer $n$, the Riemann zeta function satisfies

$$
\zeta(1-n)=-\frac{B_{n}}{n}
$$

The prime $p$ divides the denominator of $B_{n}$ in lowest form if and only if $p-1$ divides $n$, by an 1840 result of Clausen and von Staudt. Let's assume $p-1$ does not divide $n$, since otherwise it's known that the relevant eigenspace $A^{(1)}$ $=A^{(p-n)}$ is 0 .

For $m \equiv n \bmod p-1$, Kummer showed in 1851 that

$$
\zeta(1-m) \equiv \zeta(1-n) \bmod p \text {. }
$$

This explains why our criterion for regularity requires only indices $n<p$. Even better, if $m \equiv n \bmod p^{j-1}(p-1)$ for some $j \geq 1$, then we have

$$
\left(1-p^{m-1}\right) \zeta(1-m) \equiv\left(1-p^{n-1}\right) \zeta(1-n) \bmod p^{j} .
$$

Fixing an even integer $k$, these congruences imply the existence of a continuous $\mathbb{Z}_{p}$-valued function $L_{p}\left(\omega^{k}, s\right)$ of a $p$-adic variable $s \in \mathbb{Z}_{p}$ satisfying

$$
L_{p}\left(\omega^{k}, 1-n\right)=\left(1-p^{n-1}\right) \zeta(1-n)
$$

for all $n \equiv k \bmod p-1$. Here, as before, $\omega$ denotes the $p$-adic character $\omega:(\mathbb{Z} / p \mathbb{Z})^{\times} \rightarrow \mathbb{Z}_{p}^{\times}$.

The $p$-adic $L$-functions $L_{p}\left(\omega^{k}, s\right)$ were constructed by Kubota and Leopoldt. Their values at other nonnegative integers are similarly given by special values of Dirichlet $L$-functions of complex-valued characters of $\Delta$. The Riemann zeta function is the Dirichlet $L$-function for the trivial character.

\section{The Iwasawa Main Conjecture}

The Iwasawa main conjecture describes the characteristic ideal of $X_{\infty}^{(p-k)}$ for an odd prime $p$ and an even integer $k$ in terms of the $p$-adic $L$-function $L_{p}\left(\omega^{k}, s\right)$. As $X_{\infty}^{(1)}$ is trivial since $A^{(1)}=0$, let us suppose that $k \not \equiv 0 \bmod p-1$.

Iwasawa showed that $L_{p}\left(\omega^{k}, s\right)$ is determined on $s \in$ $\mathbb{Z}_{p}$ by a unique power series $f_{k} \in \Lambda$ satisfying

$$
f_{k}\left((1+p)^{s}-1\right)=L_{p}\left(\omega^{k}, s\right) .
$$

Here, we've taken the variable $T$ to correspond to $\gamma-1$ for the topological generator $\gamma$ of $\Gamma$ that raises all roots of unity of $p$-power order to the power $1+p$, and $(1+p)^{s}$ is the limit of the sequence of $(1+p)^{s_{n}}$ for $S_{n} \in \mathbb{Z}$ satisfying $s_{n} \equiv s \bmod p^{n} \mathbb{Z}_{p}$.

The following conjecture of Iwasawa's, formulated in the late 1960s, was given a proof by Mazur and Wiles in a 1984 paper [MW].
Theorem (Iwasawa main conjecture, Mazur-Wiles). For any even integer $k \neq 0 \bmod p-1$, we have

$$
\operatorname{char}\left(X_{\infty}^{(p-k)}\right)=\left(f_{k}\right) .
$$

The main conjecture implies that the highest power of $p$ dividing $L_{p}\left(\omega^{k}, s\right)$ is also the order of the largest quotient of $X_{\infty}^{(p-k)}$ upon which $T$ acts as $(1+p)^{s}-1$. Taking $s=0$, the main conjecture gives the order of $A^{(p-k)}$; it does not, however, tell us the isomorphism class (though refinements do exist).

The main conjecture can also be formulated in terms of the $p$-ramified Iwasawa module $\mathfrak{X}_{\infty}=\operatorname{Gal}\left(M_{\infty} / F_{\infty}\right)$ for $M_{\infty}$ the union of the maximal abelian $p$-extensions of the $F_{n}$ ramified only at the unique primes $\left(1-\zeta_{p^{n+1}}\right)$ over $p$. This version of the main conjecture asserts that

$$
\operatorname{char}\left(\mathfrak{X}_{\infty}^{(k)}\right)=\left(g_{k}\right),
$$

where $g_{k}$ is given by the change of variables $g_{k}(T)=$ $f_{k}\left((1+p)(1+T)^{-1}-1\right)$. The equivalence follows from an Iwasawa-theoretic version of Kummer duality.

In [Iw2], Iwasawa proved his main conjecture assuming that $A^{(k)}=0$. In this case, the equality of characteristic ideals becomes an isomorphism

$$
X_{\infty}^{(p-k)} \cong \Lambda /\left(f_{k}\right) .
$$

It's worth understanding how Iwasawa's argument goes, as through it one obtains a form of the main conjecture free from $L$-functions asserting an equality of characteristic ideals of Iwasawa modules coming from unit and class groups.

Iwasawa studied the image of the cyclotomic units inside the local units of the completion at the prime over $p$, working up the tower of completions at $p$ of the fields $F_{n}$ by considering sequences of elements compatible under norm maps. The $\Lambda$-module $\mathcal{U}_{\infty}$ of norm-compatible sequences of local units contains submodules $\mathscr{\varphi}_{\infty}$ and $C_{\infty}$ generated by the sequences of global units and cyclotomic units, respectively.

Class field theory provides an exact sequence

$$
0 \rightarrow \mathscr{\wp}_{\infty} / \mathcal{C}_{\infty} \rightarrow \mathcal{U}_{\infty} / \mathcal{C}_{\infty} \rightarrow \mathfrak{X}_{\infty} \rightarrow X_{\infty} \rightarrow 0 .
$$

This in turn yields an exact sequence with each module replaced by its $\omega^{k}$-eigenspace under $\Delta$. If $X_{\infty}^{(k)}=0$, then the class number formula can be used to see that $\mathscr{\wp}_{\infty}^{(k)}=C_{\infty}^{(k)}$ as well. The four-term exact sequence therefore reduces to an isomorphism

$$
\mathcal{U}_{\infty}^{(k)} / C_{\infty}^{(k)} \cong \mathfrak{X}_{\infty}^{(k)} .
$$

Iwasawa then obtains that $\mathfrak{X}_{\infty}^{(k)} \cong \Lambda /\left(g_{k}\right)$ from the following unconditional theorem, which amounts to a $p$-adic regulator computation on cyclotomic units. 
Theorem (Iwasawa). There is an isomorphism of $\Lambda$-modules

$$
\mathcal{U}_{\infty}^{(k)} / C_{\infty}^{(k)} \cong \Lambda /\left(g_{k}\right) .
$$

As characteristic ideals are multiplicative in exact sequences of finitely generated, torsion $\Lambda$-modules, this also tells us unconditionally that the main conjecture is equivalent to the equality

$$
\operatorname{char}\left(\mathscr{\wp}_{\infty}^{(k)} / C_{\infty}^{(k)}\right)=\operatorname{char}\left(X_{\infty}^{(k)}\right)
$$

in which no $L$-functions appear.

\section{Modular Forms}

One might say that the theme of the work of Ribet and Mazur-Wiles is that the study of the geometry of varieties over $\mathbb{Q}$ can be used to solve arithmetic questions. Specifically, their work makes use of modular curves and congruences between modular forms. The Galois representations attached to modular forms are two-dimensional, presenting a natural next class of objects to study beyond the onedimensional abelian characters of class field theory. We embark upon another brief tour.

The group of matrices in $\mathrm{GL}_{2}(\mathbb{R})$ with positive determinant acts by Möbius transformations on the upper halfplane $\mathbb{W}$ of complex numbers with positive imaginary part. A modular curve is a quotient of $\mathbb{T}$ by the action of a subgroup of $\mathrm{SL}_{2}(\mathbb{Z})$ that is determined by congruences among its entries. This quotient can be compactified by adding in the equivalence classes of the cusps, which are the rational numbers and infinity.

A modular form $f$ is a holomorphic function of $\mathbb{t}$ that transforms under a congruence subgroup $\Gamma$ (in the standard notation, but not to be confused with the Galois group appearing in Iwasawa theory) in a manner prescribed by its "weight" $k$, and which is bounded and holomorphic at the cusps. Specifically, if $\left(\begin{array}{ll}a & b \\ c & d\end{array}\right) \in \Gamma$, then

$$
f\left(\frac{a z+b}{c z+d}\right)=(c z+d)^{k} f(z) .
$$

A modular form is a cusp form if it is zero at all cusps.

If $\left(\begin{array}{ll}1 & 1 \\ 0 & 1\end{array}\right) \in \Gamma$, then $f(z+1)=f(z)$, so $f$ has a Fourier expansion about the cusp at $\infty$ of the form

$$
f=\sum_{n=0}^{\infty} a_{n}(f) q^{n}
$$

with $q=e^{2 \pi i z}$ for $z \in \mathbb{H}$. If $f$ is a cusp form, then $a_{0}(f)=0$.

There are Hecke operators $T_{n}$ for each $n \geq 1$ that act on modular forms by summing over the action of representatives of the double coset $\Gamma\left(\begin{array}{ll}1 & 0 \\ 0 & n\end{array}\right) \Gamma$ as a union of right cosets. A modular form is an eigenform if it is a simultaneous eigenform for all Hecke operators. If an eigenform is normalized so that $a_{1}(f)=1$, then $T_{n}(f)=a_{n}(f) f$ for all $n \geq 1$. The Fourier coefficients $a_{n}(f)$ of a normalized eigenform $f$ are algebraic numbers that are integral for $n \geq 1$, and the coefficient field they generate is a number field.

Eisenstein series form a class of modular forms that are not cusp forms. For instance, for a positive even integer $k \geq 4$, we have an Eisenstein series

$$
E_{k}=-\frac{B_{k}}{2 k}+\sum_{n=1}^{\infty} \sum_{d \mid n} d^{k-1} q^{n}
$$

which is an eigenform of weight $k$ for $\Gamma=\mathrm{SL}_{2}(\mathbb{Z})$ itself. When an odd prime $p$ divides $\zeta(1-k)=-B_{k} / k$, the constant term of $E_{k}$ is zero modulo $p$. In this case, the reduction of $E_{k}$ modulo $p$ may be lifted to a cuspidal eigenform with coefficients in the ring of integers of a number field. For example, $E_{12}$ is congruent modulo $691 \mathbb{Z} \llbracket q \rrbracket$ to the unique normalized cusp form

$$
q \prod_{n=1}^{\infty}\left(1-q^{n}\right)^{24}
$$

of weight 12 for $\mathrm{SL}_{2}(\mathbb{Z})$.

To a normalized cuspidal eigenform $f$ of weight $k \geq 2$, work of Shimura and Deligne attached a $p$-adic Galois representation $\rho_{f}: G_{\mathbb{Q}} \rightarrow \mathrm{GL}_{2}\left(K_{f}\right)$, with $K_{f}$ the field obtained by adjoining to $\mathbb{Q}_{p}$ the Fourier coefficients of $f$. Equivalently, it is a two-dimensional $K_{f}$-vector space $V_{f}$

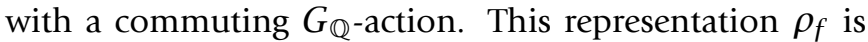
irreducible and odd (i.e., it has determinant -1 on complex conjugation), and it has the property that the trace of $\rho_{f}\left(\varphi_{\ell}\right)$ for a Frobenius element $\varphi_{\ell}$ of an unramified prime over a rational prime $\ell$ is equal to $a_{\ell}(f)$.

Inside $V_{f}$, there is a rank-two module $L_{f}$ for the valuation ring $\mathcal{O}_{f}$ of $K_{f}$ that is preserved by the $G_{\mathbb{Q}}$-action. Roughly, this says that $\rho_{f}$ can be viewed as taking values in $\mathrm{GL}_{2}\left(\mathcal{O}_{f}\right)$. So, it makes sense to reduce $\rho_{f}$ modulo the maximal ideal of $\mathcal{O}_{f}$ and talk about the resulting representation $\bar{\rho}_{f}: G_{\mathbb{Q}} \rightarrow \mathrm{GL}_{2}\left(\mathbb{F}_{q}\right)$ over the residue field $\mathbb{F}_{q}$. This residual representation is unique up to isomorphism if and only if it is irreducible. Otherwise, its isomorphism class depends upon the choice of $L_{f}$.

\section{The Method of Ribet-Mazur-Wiles}

Ribet and Mazur-Wiles employed congruences between cusp forms and Eisenstein series to construct unramified abelian extensions of cyclotomic fields. As we've noted, if $p \mid B_{k}$ for an even $k<p$, then there exists a cuspidal eigenform $f$ congruent to $E_{k}$ modulo the maximal ideal of $\mathcal{O}_{f}$. The fixed field of the kernel of the Galois representation $\rho_{f}$ is ramified only at the prime $p$. Ribet used this to construct an unramified abelian $p$-extension of $F=\mathbb{Q}\left(\zeta_{p}\right)$ on which $\Delta$ acts through $\omega^{p-k}$. By class field theory, if the extension is nontrivial, then $A^{(p-k)}$ is nonzero, which is Ribet's converse to Herbrand's theorem. 
The method works by playing two facts forced by the congruence off of each other. The first is that $f$ is ordinary in the sense that $a_{p}(f)$ is a $p$-adic unit. For ordinary forms, there exists a basis of $V_{f}$ such that $\rho_{f}$ restricted to a decomposition group at $p$ is upper triangular as a map to $\mathrm{GL}_{2}\left(\mathcal{O}_{f}\right)$. If needed, one can rescale so that the map $\phi: G_{\mathbb{Q}} \rightarrow \mathbb{F}_{q}$ to the residue field $\mathbb{F}_{q}$ given by the reduction of the lower left-hand corner of $\rho_{f}$ modulo the maximal ideal of $\mathcal{O}_{f}$ is nonzero on the larger group $G_{\mathbb{Q}}$.

The second fact is that the residual Galois representation $\bar{\rho}_{f}$ is reducible. The above basis can actually be chosen so that $\bar{\rho}_{f}$ has the form

$$
\bar{\rho}_{f}(\sigma)=\left(\begin{array}{cc}
\omega^{k-1}(\sigma) & 0 \\
\phi(\sigma) & 1
\end{array}\right)
$$

on $\sigma \in G_{\mathbb{Q}}$, viewing $\omega$ as a character of $G_{\mathbb{Q}}$. The restriction of $\phi$ to $\operatorname{Gal}(\overline{\mathbb{Q}} / F)$ is then a homomorphism that is unramified at the prime over $p$ by construction, so it factors through the Galois group of a nontrivial unramified abelian $p$-extension $H$ of $F$ that is Galois over $\mathbb{Q}$. The group $\Delta$ acts on $\mathrm{Gal}(H / F)$ compatibly with conjugation of matrices, which is to say that it acts by $\omega^{p-k}=$ $\left(\omega^{k-1}\right)^{-1}$

Mazur and Wiles generalized and refined Ribet's method in the context of Iwasawa theory in order to prove the main conjecture and its generalization to arbitrary abelian extensions of $\mathbb{Q}$. By studying the Galois actions on Jacobians of modular curves, they construct an unramified abelian extension of the field $F_{\infty}$ of all $p$-power roots of unity with Galois group a $\Lambda$-module quotient of $X_{\infty}^{(p-k)}$ that has characteristic ideal $\left(f_{k}\right)$.

Having proven that $\left(f_{k}\right) \mid \operatorname{char}\left(X_{\infty}^{(p-k)}\right)$, Mazur and Wiles apply a consequence of analytic class number formula to obtain the main conjecture. That is, Iwasawa had shown in 1972 that

$$
\sum_{j=1}^{(p-3) / 2} \lambda\left(X_{\infty}^{(p-2 j)}\right)=\sum_{j=1}^{(p-3) / 2} \operatorname{deg} f_{2 j} .
$$

From this, it follows that one divisibility for all odd eigenspaces implies the other.

Later work of Wiles gave a more streamlined perspective, casting the proof in terms of the theory of families of ordinary modular forms of Hida. That is, Wiles employed the residual representation of a Galois representation $\rho_{\mathcal{F}}: G_{\mathbb{Q}} \rightarrow \mathrm{GL}_{2}\left(\square_{f}\right)$ attached to a $p$-adically continuously varying family $\mathcal{F}$ of ordinary cuspidal eigenforms congruent to a family of Eisenstein series, where $\nabla_{f}$ is a finite local $\Lambda$-algebra.

\section{The Method of Euler Systems}

Work of Thaine and Kolyvagin led to a new and more explicit, though technically complex, approach to the main conjecture, which Rubin completed to a full proof. The method uses what Kolyvagin termed an Euler system, a first example of which consists of cyclotomic units in abelian extensions of $\mathbb{Q}$. This system of elements is used to bound the order of an even eigenspace of the $p$-part of the class group by the order of an eigenspace of the quotient of the global units by the cyclotomic units.

The key property is a norm compatibility from $\mathbb{Q}\left(\zeta_{N \ell}\right)$ to $\mathbb{Q}\left(\zeta_{N}\right)$ for $p$ dividing $N$ and a prime $\ell$. Explicitly, if $\ell \nmid N$, one has

$$
N_{\mathbb{Q}\left(\zeta_{N \ell}\right) / \mathbb{Q}\left(\zeta_{N}\right)}\left(1-\zeta_{N \ell}\right)=\frac{1-\zeta_{N}}{1-\zeta_{N}^{\ell-1}} .
$$

With this relation, one applies a Galois-theoretic derivative construction to elements $1-\zeta_{N \ell}$ for good choices of primes $\ell$ congruent to \pm 1 modulo a sufficiently high power of $p$ to obtain field elements that are powers of chosen nonprincipal ideals.

Up the cyclotomic tower, the method of Euler systems shows that

$$
\operatorname{char}\left(X_{\infty}^{(k)}\right) \mid \operatorname{char}\left(\mathscr{\wp}_{\infty}^{(k)} / C_{\infty}^{(k)}\right)
$$

for even $k \in \mathbb{Z}$. This is equivalent to the opposite divisibility to that of Mazur-Wiles, and again the analytic class number formula yields equality.

\section{Totally Real and CM Fields}

The Iwasawa main conjecture generalizes directly from $\mathbb{Q}$ to totally real fields, those number fields with only real archimedean embeddings. The relevant $p$-adic $L$-functions were separately constructed by Deligne and Ribet, CassouNoguès, and Barsky. Wiles proved a main conjecture for odd eigenspaces of the unramified Iwasawa module $X_{\infty}$ over the cyclotomic $\mathbb{Z}_{p}$-extension $F_{\infty}$ of an abelian extension $F$ of a totally real field using Galois representations attached to Hilbert modular forms [Wi]. Greenberg has conjectured that the even eigenspaces of $X_{\infty}$ are finite.

The existence of an Euler system in abelian extensions of totally real fields was conjectured by Stark and Rubin, but it is still unproven. This is related to Hilbert's twelfth problem, or Kronecker's Jugendtraum, of an explicit class field theory over totally real fields. In contrast, abelian extensions of imaginary quadratic fields contain analogues of cyclotomic units called elliptic units that do form an Euler system. In this case, there is an explicit form of class field theory arising from the theory of complex multiplication (CM) of elliptic curves that we'll discuss. Elliptic units are generated by values at torsion points of a theta function that is a meromorphic function on a CM elliptic curve.

In 1977, Coates and Wiles proved an analogue of Iwasawa's theorem on local units modulo cyclotomic units for 
an imaginary quadratic field $K$ with $h_{K}=1$ [CW]. Choosing a split prime $p$ with $p \mathcal{O}_{K}=\mathfrak{p} \overline{\mathfrak{p}}$, their theorem states that the quotient of the local units at $\mathfrak{p}$ modulo elliptic units up a $\mathbb{Z}_{p}$-extension of $K$ ramified only at $\mathfrak{p}$ is isomorphic to the quotient of the one-variable Iwasawa algebra by a power series corresponding to a $p$-adic $L$-function constructed by Katz.

The compositum of $\mathbb{Z}_{p}$-extensions of an imaginary quadratic field $K$ has Galois group $\mathbb{Z}_{p}^{2}$. Its Iwasawa algebra is a power series ring in two variables over $\mathbb{Z}_{p}$. The unramified Iwasawa module $X_{\infty}$ over the corresponding $\mathbb{Z}_{p}^{2}$-extension of an abelian extension of $K$ is conjecturally small enough to have unit characteristic ideal. The main conjecture here compares $X_{\infty}$ and the quotient of global units by elliptic units. It was proven by Rubin using the method of Euler systems in 1991 [Ru]. If $p$ splits in $K$, one can instead replace $X_{\infty}$ with a larger Iwasawa module that allows ramification at exactly one of the two primes over $p$. This gives an equivalent form involving a two-variable Katz $p$-adic $L$-function.

In 1994, Hida and Tilouine gave an alternate proof of the specialization of this conjecture to an anticyclotomic $\mathbb{Z}_{p}$-extension using an approach closer to that of MazurWiles. Their work extends to analogues of imaginary quadratic fields over totally real fields known as CM fields for which one once again has no known Euler system to employ. More recently, progress has been made on a divisibility in a general main conjecture over CM fields, in particular by Hsieh.

\section{Elliptic Curves and BSD}

Elliptic curves over number fields provide a next step beyond the theory of multiplicative groups that we've been in effect describing. In this setting, the arithmetic objects that replace class groups are known as Selmer groups. We run quickly through the arithmetic theory of elliptic curves, from the basic theory to deep results and a famous conjecture, before we begin passing up towers.

A complex elliptic curve $E$ is defined by an equation

$$
y^{2}=x^{3}+a x+b
$$

with $a, b \in \mathbb{C}$ and $4 a^{3}+27 b^{2} \neq 0$ to ensure smoothness. More precisely, it is the projective curve of genus one defined by the homogenization of the above polynomial. Effectively, this means adding a single point $\infty$ at infinity.

The set $E(\mathbb{C})$ of points of $E$ with complex coordinates has an abelian group law with $\infty$ as its identity. It is given by drawing a line between two points $P$ and $Q$ and declaring the third point of the line in $E(\mathbb{C})$ to be $-P-Q$, taking multiplicity into account. We'll assume that $a, b \in \mathbb{Q}$, which is to say that $E$ is rational. The Mordell-Weil group $E(F)$ of points with coordinates in a number field $F$ is then a finitely generated subgroup of $E(\mathbb{C})$.
The group $E(\overline{\mathbb{Q}})$ has a canonical action of $G_{\mathbb{Q}}$ via the action on coordinates. A point of $E(\mathbb{C}) \cong(\mathbb{R} / \mathbb{Z})^{2}$ is said to be $n$-torsion if it has order dividing $n$. The group $E[n]$ of all $n$-torsion points is a subgroup of $E(\overline{\mathbb{Q}})$ isomorphic to $(\mathbb{Z} / n \mathbb{Z})^{2}$.

A rational elliptic curve $E$ has a ring $\mathcal{O}$ of endomorphisms consisting of nonzero $\mathbb{Q}$-rational maps $E \rightarrow E$ taking $\infty$ to itself. Among these are the morphisms given by multiplication by integers using the group law of $E$. If $\mathcal{O} \neq \mathbb{Z}$, then $\mathcal{O}$ is a finite index subring of the integer ring of an imaginary quadratic field. In this case, we say that $E$ has $\mathrm{CM}$ by $\mathcal{O}$.

The cohomology of a group $G$ with coefficients in a module $M$ for the group ring $\mathbb{Z}[G]$ is a sequence of abelian groups $H^{i}(G, M)$ that allows one to study the group action using the tools of homological algebra. For Galois groups, the closely related theory of Galois cohomology is a crucial tool in Iwasawa theory. Many theorems of class field theory can be phrased in terms of duality in Galois cohomology, and abelian groups of arithmetic interest can be encoded in cohomology.

For a number field $F$, the group $E(F) / n E(F)$ is contained in $H^{1}(\operatorname{Gal}(\overline{\mathbb{Q}} / F), E[n])$. In fact, it is contained in a smaller subgroup of cohomology classes that are unramified at all but finitely many primes and partially vanish locally at the remaining primes. The direct limit over $n$ of these subgroups of $H^{1}(\operatorname{Gal}(\overline{\mathbb{Q}} / F), E[n])$ is the Selmer $\operatorname{group} \operatorname{Sel}_{E}(F)$ we wish to study.

The most important thing to know about the Selmer group is that it intertwines the Mordell-Weil group with a mysterious, conjecturally finite group called the Shafarevich-Tate group $\amalg_{E}(F)$ of $E$. That is, there is an exact sequence:

$$
0 \rightarrow E(F) \otimes \mathbb{Q} / \mathbb{Z} \rightarrow \operatorname{Sel}_{E}(F) \rightarrow \amalg_{E}(F) \rightarrow 0 .
$$

This is analogous to what happens with cohomology with coefficients in roots of unity: in that case, the unit and class groups get wrapped up together.

The intertwining of $E(\mathbb{Q})$ and $\amalg_{E}(\mathbb{Q})$ is also reflected in analytic formulas. One can construct an $L$-series for $E$ from the data of the number of $\mathbb{F}_{p}$-points of $\bmod p$ reductions of a minimal equation for $E$. It has analytic continuation to $\mathbb{C}$ by the modularity of rational elliptic curves proven by Wiles, Taylor-Wiles, and Breuil-ConradDiamond-Taylor, which tells one that $E$ has an associated cuspidal eigenform with the same $L$-series.

The Birch and Swinnerton-Dyer conjecture, or BSD, was formulated in 1965 and is one of the Clay Math Institute's Millennium Problems.

Conjecture (Birch and Swinnerton-Dyer). The order of vanishing of the $L$-function $L(E, s)$ at $s=1$ is equal to the rank of $E(\mathbb{Q})$. 
BSD has a refined form that links the leading term of $L(E, s)$ in its Taylor expansion about $s=1$ to the orders of the torsion subgroup $E(\mathbb{Q})_{\text {tor }}$ of Mordell-Weil and of $\amalg_{E}(\mathbb{Q})$. The conjectural BSD formula has the form

$$
\frac{L^{(r)}(E, 1)}{r !}=\frac{\left|\amalg_{E}(\mathbb{Q})\right| \Omega_{E} R_{E} \prod_{\ell} c_{\ell}}{\left|E(\mathbb{Q})_{\text {tor }}\right|^{2}},
$$

where $r$ is the order of vanishing, $R_{E}$ is a regulator related to the heights of rational points, the quantity $\Omega_{E}$ is a real period, and each $c_{\ell}$ for a prime $\ell$ is the number of components of a certain $\bmod \ell$ reduction of $E$, all but finitely many being 1 .

Much of the progress on BSD to date employs Iwasawa theory. For instance, the first major result that serves as theoretical evidence for BSD was due to Coates-Wiles. As a consequence of their theorem on local units modulo elliptic units, they proved that if $r=0$ and $E$ has CM by a subring of $\mathcal{O}_{K}$ for $K$ with $h_{K}=1$, then $E(\mathbb{Q})$ is finite.

\section{Iwasawa Theory of Elliptic Curves}

We turn to the question of how Selmer groups of a rational elliptic curve $E$ grow in the cyclotomic $\mathbb{Z}_{p}$-extension $\mathbb{Q}_{\infty}$ of $\mathbb{Q}$. This amounts to studying the finitely generated $\Lambda$ module

$$
\mathfrak{X}_{E}=\operatorname{Hom}\left(\operatorname{Sel}_{E}\left(\mathbb{Q}_{\infty}\right), \mathbb{Q}_{p} / \mathbb{Z}_{p}\right)
$$

that is the Pontryagin dual of the direct limit $\operatorname{Sel}_{E}\left(\mathbb{Q}_{\infty}\right)\left[p^{\infty}\right]$ of $p$-power torsion subgroups of the Selmer groups $\operatorname{Sel}_{E}\left(\mathbb{Q}_{n}\right)$.

The elliptic curve $E$ has good reduction at $p$ if its $\bmod$ $p$ reduction $E_{p}$ is nonsingular, and it is then ordinary if $E_{p}$ has a point of order $p$. For such elliptic curves, Mazur and Swinnerton-Dyer constructed a $p$-adic $L$-function $L_{p}(E, s)$ interpolating values of $L(E, S)$ up to certain Euler factors, and again it is determined by a power series $\mathcal{L}_{E}$. Mazur then formulated the following main conjecture.

Conjecture (Main conjecture for elliptic curves). Suppose that $E$ has good ordinary reduction at $p$ and that $E(\overline{\mathbb{Q}})[p]$ is an irreducible $G_{\mathbb{Q}}$-representation. Then $\mathfrak{X}_{E}$ is $\Lambda$-torsion and

$$
\operatorname{char}\left(\mathfrak{X}_{E}\right)=\left(\mathcal{L}_{E}\right) \text {. }
$$

For elliptic curves with $\mathrm{CM}$, this is equivalent to the main conjecture for imaginary quadratic fields and split primes $p$ proven by Rubin. The general divisibility $\operatorname{char}\left(\mathfrak{X}_{E}\right) \mid\left(\mathcal{L}_{E}\right)$ was proven by Kato via the method of Euler systems [Ka]. Kato's Euler system is constructed using cohomological products formed from pairs of Siegel units on a modular curve parameterizing $E$, first studied by Beilinson. The other divisibility was proven under fairly mild hypotheses by Skinner and Urban using Galois representations attached to automorphic forms on the unitary group $\mathrm{GU}(2,2)$ [SU]. With no analytic class number formula that can be used in this setting, one needs both methods.
We mention a bit of what's known for elliptic curves with good supersingular (i.e., nonordinary) reduction. For $p \geq 5$, there are in this case not one but two Selmer groups constructed by Kobayashi and two $p$-adic $L$-functions constructed by Pollack. The corresponding main conjecture was proven by Rubin and Pollack for CM curves in 2004. In this case, $p$ does not split in $K$, and the main conjecture is closely related to Rubin's main conjecture without $L$ functions for imaginary quadratic fields. The main conjecture for non-CM curves has recently been proven by Wan under a hypothesis on the congruence subgroup. Sprung has additionally treated the prime 3 , in particular employing work of Kim and Lei in the formulation.

The main conjecture for elliptic curves implies a $p$-adic analogue of BSD of Mazur-Tate-Teitelbaum that relates the rank of $E(\mathbb{Q})$ to the order of vanishing of $L_{p}(E, s)$ at 1 . As $L(E, s)$ is complex analytic and $L_{p}(E, s)$ is $p$-adic analytic, the derivatives are not clearly related, and neither form of BSD obviously implies the other in the case of positive rank.

The order $r$ of vanishing of $L(E, s)$ at $s=1$ is known as the analytic rank of $E$, and the actual rank of $E(\mathbb{Q})$ is known as the algebraic rank. Kolyvagin used an Euler system of Heegner points and a theorem of Gross and Zagier to prove that if the analytic rank of $E$ is $r \leq 1$, then the algebraic rank is $r$ and $\amalg_{E}(\mathbb{Q})$ is finite. Both the converse to this and the BSD formula follow from the main conjecture for $r=0$. Recent work of Skinner and of Zhang implies a converse for $r=1$ under mild hypotheses, and for $r=1$ significant progress has been made towards the BSD formula as well, particularly in work of Jetchev, Skinner, and Wan.

\section{Recent Directions}

Iwasawa theory extends to study the growth of other arithmetic objects attached to Galois representations in towers of number fields. Beginning in the late 1980s, Greenberg proposed main conjectures for a whole host of ordinary motivic Galois representations, and even continuously $p$ adically varying families thereof. Since then, main conjectures have been extended to more general towers, nonordinary families, finer-grained analogues, and even characteristic $p$ base fields. Recent developments have seen considerable progress on methods of proof of both divisibilities. Here's a sampling.

The early part of the new millennium saw the development of Iwasawa theory over towers of number fields with Galois groups that are isomorphic to subgroups of $\mathrm{GL}_{n}\left(\mathbb{Z}_{p}\right)$ for some $n$. The breakthrough came in a paper of Coates, Fukaya, Kato, Sujatha, and Venjakob containing a noncommutative main conjecture for elliptic curves. Invariants playing the roles of characteristic ideals and $p$ adic $L$-functions lie in a first $K$-group of a localization of 
the noncommutative Iwasawa algebra. Soon after, Fukaya and Kato formulated a remarkably general noncommutative main conjecture that relates closely to the equivariant Tamagawa number conjecture of Burns and Flach which in turn generalized a conjecture of Bloch and Kato on special values of $L$-functions.

At the start of this decade, a noncommutative main conjecture for totally real fields was proven through work of Ritter and Weiss, Burns, and Kakde, assuming Iwasawa's conjecture on the vanishing of the $\mu$-invariant. The key step is the verification of congruences among DeligneRibet $p$-adic $L$-functions over different totally real fields to reduce to the main conjecture proven by Wiles.

The work of Skinner-Urban has inspired a rush of new theorems on divisibilities in main conjectures, with recent progress on the construction of Galois representations attached to automorphic forms in the sense of the Langlands program providing a boon for the area. The construction of $p$-adic $L$-functions that interpolate special values of complex $L$-functions is a whole industry unto itself and often proceeds via distributions formed out of modular symbols or computations of local integrals.

Euler systems have long had a reputation as difficult to construct that has only recently begun to soften. An approach to construct them via geometric methods starting from cycles on varieties was initiated in work of Bertolini, Darmon, Prasanna, and Rotger and carried further in work of Loeffler, Zerbes, Lei, Kings, and Skinner. One typically computes complex and $p$-adic regulators to prove nonvanishing of Euler systems and relate them with ( $p$-adic) $L$ functions.

\section{Beyond Mazur-Wiles}

I end with a brief discussion of a deeper relationship between the geometry of modular curves and the arithmetic of cyclotomic fields. In a 2011 paper, I formulated a conjecture relating relative homology classes $\{\alpha \rightarrow \beta\}$ of paths between cusps on a modular curve, taken here to be $X_{1}(p)$, and cup products $x \cup y$ of cyclotomic units in a Galois cohomology group that agrees with $A^{-}$modulo $p$. In fact, there is a simple explicit map

$$
\left\{\frac{a}{c} \rightarrow \frac{b}{d}\right\} \mapsto\left(1-\zeta_{p}^{c}\right) \cup\left(1-\zeta_{p}^{d}\right)
$$

taking one set of elements to the other (for $a d-b c=$ 1 and $p \nmid c d$ ). On a certain Eisenstein quotient of the plus part of homology, I conjectured this to provide an inverse to a canonical version of the map that appeared in the proof of Ribet's theorem.

Up the cyclotomic tower, this yields what can be viewed as a refinement of the Iwasawa main conjecture. That is, it provides not only an equality of characteristic ideals, but an isomorphism given by a recipe on special elements.
Fukaya and Kato proved a major result in this direction in which the derivative of the $p$-adic $L$-function plays a crucial and potentially unavoidable intermediate role. Their result implies the conjecture for $p<2^{31}$.

Fukaya, Kato, and I expect this to be a special case of a general phenomenon of the geometry and topology of locally symmetric spaces of higher dimension informing the arithmetic of Galois representations attached to lowerdimensional automorphic forms. This begs the question of its elliptic curve analogue, which is but one of a wealth of intriguing possibilities for future directions in Iwasawa theory.

\section{References}

[CW] Coates J, Wiles A, On the conjecture of Birch and Swinnerton-Dyer, Invent. Math., (39): 223-251, 1977. MR0463176

[FW] Ferrero B, Washington L, The Iwasawa invariant $\mu_{p}$ vanishes for abelian number fields, Ann. of Math., (109): 377 395, 1979. MR528968

[Iw1] Iwasawa $\mathrm{K}$, On $\Gamma$-extensions of algebraic number fields, Bull. Amer. Math. Soc., (65): 183-226, 1959. MR0124316

[Iw2] Iwasawa K, On $p$-adic L-functions, Ann. of Math., (89): 198-205, 1969. MR0269627

[Ka] Kato K, $p$-adic Hodge theory and values of zeta functions of modular forms, Cohomologies $p$-adiques et applications arithmétiques, III, Astérisque, (295): 117-290, 2004. MR2104361

[MW] Mazur B, Wiles A, Class fields of abelian extensions of $\mathbb{Q}$, Invent. Math., (76): 179-330, 1984. MR742853

[Ri] Ribet K, A modular construction of unramified $p$ extensions of $\mathbb{Q}\left(\mu_{p}\right)$, Invent. Math., (34) 151-162, 1976. MR0419403

[Ru] Rubin K, The "main conjectures" of Iwasawa theory for imaginary quadratic fields, Invent. Math., (103): 25-68, 1991. MR1079839

[SU] Skinner C, Urban E, The Iwasawa main conjectures for $\mathrm{GL}_{2}$, Invent. Math., (195): 1-227, 2014. MR3148103

[Wi] Wiles A, The Iwasawa conjecture for totally real fields, Ann. of Math., (131): 493-540, 1990. MR1053488

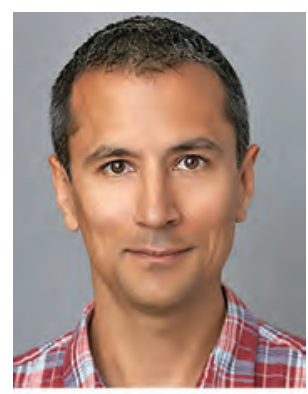

Romyar Sharifi

\section{Credits}

Opening photo is courtesy of Getty Images.

Author photo is by Joshua M. Shelton. 


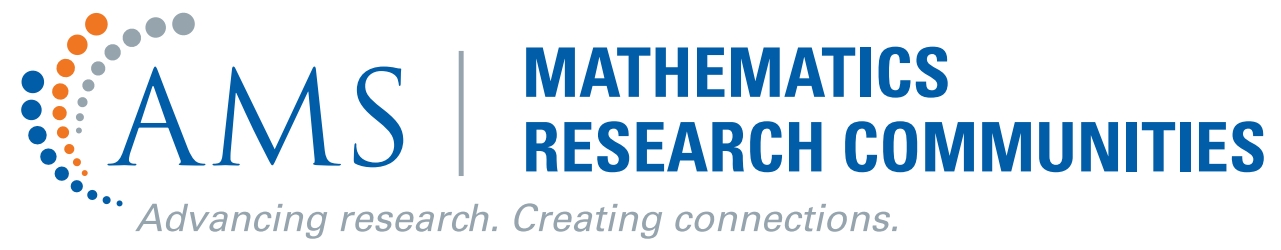

To learn more about the American Mathematical Society's Mathematics Research Communities

and how they help early-career mathematicians develop cohorts for collaborative research:

Visit the MRC Booth at the Joint Mathematics Meetings.

We are located in the AMS booth at the end of the 500 aisle in the Baltimore Convention Center.

WWW.AMS.ORG/MRC

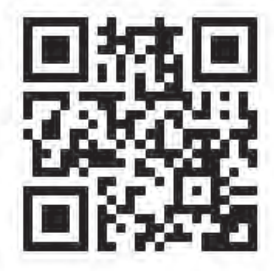

AMS MMELCAN 
The Early Career Section is a new community project, featured here in the Notices. This column will provide information and suggestions for graduate students, job seekers, junior academics of all types, and those who mentor them Angela Gibney is the editor of the Early Career section. Angela Gibney will serve as the editor of this section.

\section{Introducing the Notices Early Career Section}

\section{A. Gibney}

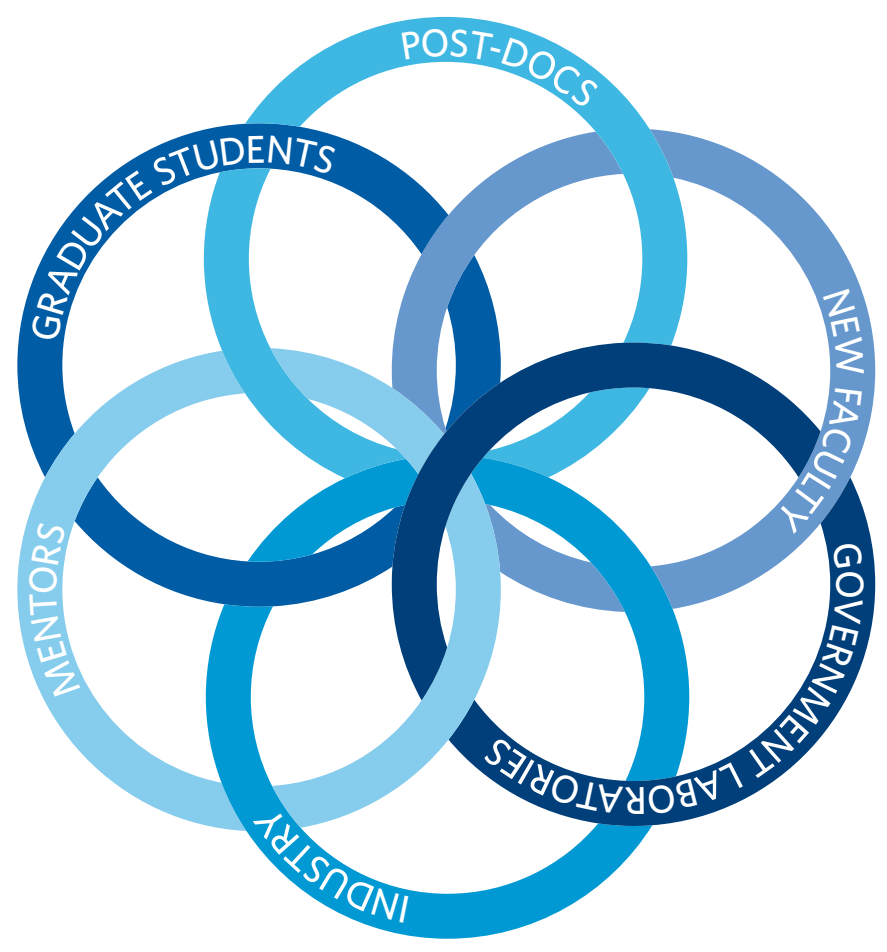

Angela Gibney is professor of mathematics at Rutgers University and associate editor of Notices. Her email address is angela.gibney Qgmai1.com.

For permission to reprint this article, please contact: reprint -permission@ams.org.

DOI: http://dx.doi.org/10.1090/noti1782
It is well understood that the path to an interesting career is just not easy. The road, while at times adventurous, is generally long, sometimes hard, and often hard to find. How can you manage it, to arrive in one piece?

The Early Career Section is a new community project, featured here in the Notices. This column will provide information and suggestions for graduate students, job seekers, junior academics of all types, and those who mentor them. We will consider different aspects of the profession, arranged in themes for each issue, with articles written by mathematicians about topics surrounding that theme. As a first contribution, Rob Lazarsfeld has written a piece about mathematical journaling.

In February we consider issues surrounding the world of research. One of the biggest steps taken by graduate students in this realm is to select a thesis advisor, and Robert Lipshitz will discuss this. Ellen Eischen will weigh in on strategies for moving ahead in one's research, and Chris Woodward about getting new ideas for projects. Kelly Jabbusch will write about Mathematical Reviews ${ }^{\circledR}$, which can be a tremendous tool for learning about the literature, and also a way to contribute to the community. Sam Payne and Amanda Folsom will discuss aspects of doing research with undergraduate students, describing the program SUMRY at Yale University.

We chose March for the "Writing Issue" as it is generally around the time graduate students are starting to finish their thesis and prepare the corresponding paper for publication. It may seem counterintuitive, but writing is one of the central aspects of the work we do as mathematicians. John Etnyre has contributed a piece about writing an introduction to help you maximize the chances people will appreciate your work. Julia Hartmann will address aspects of writing up one's work and submitting it to a journal, and Arend Bayer will discuss referee reports, both writing them 
and reading and responding to them. Joe Silverman will share advice about writing a book, and Jordan Ellenberg about writing math for the general public.

In April, those who plan to go on the non-academic job market generally start the job application process. Often there is a lack of knowledge about options available and ways to make connections with employers. The April issue is about nonacademic math careers and is meant to be a resource for the community of job seekers and those who advise them. Jesse Johnson will write a piece on transitioning to jobs outside of academia. Jim Gatheral will write about careers in financial engineering, Petter Kolm describes careers in quantitative finance, and Emilie Purvine will write about careers at the National Labs.

In May we begin to look ahead to summer break, and many will be thinking about how to get the most out of this big block of free time. Some graduate students will have a choice of summer schools and conferences, and for many junior researchers, it may be a good idea to think about organizing such activities. We plan to take on these topics with articles about how to find good conferences and summer schools for the next year (how to look, how to apply, how to choose what is best); and why folks who are in their early careers could benefit from organizing activities for others. Daniel Krashen will write an article on the nuts and bolts of conference organization in general. Brendan Hassett will contribute a piece about opportunities at the math institutes. We also plan to have an article about how to use free time productively.

Deep into summer break when the June/July issue of the Notices arrives in the mail, people are reminded that they need to make a plan for the coming academic year. This edition will be devoted to getting ready for the academic job market, and applying for grants. Holly Krieger will write about the importance of a good website and online presence, as well as about seeking a job at a research focused institution. Linda Chen will write about seeking a job at a liberal arts institution. John Etnyre will discuss writing grant proposals. Diane Maclagan will write about two-body issues and the job search.

In August many of us are looking ahead to the academic year, and our theme for this issue is on the teaching aspect of our careers. Jessica Sidman will write about inclusive teaching and overcoming student anxiety. Hannah Bennett will write a piece about the Flipped Classroom and Inquiry Based Learning, and Jen Bowen will discuss the supervision of senior theses. Jasmine $\mathrm{Ng}$ will write about teaching at a community college. We also plan to address issues surrounding how to decide what you should teach, how to decide whether or not to revamp the class you've been teaching, and dealing with teaching evaluations.

We depend on letters of recommendation at every stage in our academic careers and for this reason and so many others, it is crucial to develop meaningful and productive professional relationships with experts in our fields. In
September many people either ask, or are asked for letters of recommendation for the coming job market, and so we chose September to feature an issue about how to be a mentor, maintain a good relationship with your mentor, and carry out your mentoring duties. Ken Ono and Bernd Sturmfels will both contribute articles about advising. Ken Ribet will write with general advice to graduate students. Dawei Chen will write about reaching out to experts in your field.

October is our "Work-Life Balance Issue." We will discuss strategies to support your career when non-work situations become more demanding. One of the most important things to do in your early career is hang in there and not quit during the long waiting periods such as when finishing your PhD, waiting for job interviews, and tenure and promotion. We are going to give some practical advice on these issues.

The November issue will be focused on planning ahead for the Joint Meetings. In particular we discuss how to prepare for job market and special session activities. Dawei Chen and Alec Kercheval will write about giving talks of various types. Katelynn Kochalski will give advice on preparing for the Employment Center and Joint Meetings interviews for tenure track jobs.

In December, in our "Best Practices Issue," we plan to look back and give kudos to programs and departments that are doing things that we'd like to see emulated throughout the country. We'd like to disseminate those best practices to make them available for other departments to copy and to give chairs of departments information to take to their administrators in order to gain resources to improve their departments. We plan to highlight ideas that departments have come up with to improve working conditions and work/life balance.

Of course there are many other sources of good information along these lines (and far too many to list here!). We hope this section of the Notices will simply provide more perspectives, from people in the community who are interested in helping you get where you want to go. 


\section{EARLY CAREER}

\section{Journaling}

\section{Robert Lazarsfeld}

A later installment of this series, in March, will focus on writing up mathematical research. Here I want to advocate the benefits of regularly writing it down. I don't refer to the universally recognized importance of committing to paper all the details of one's arguments. Rather I want to suggest that maintaining a record of mathematical activity can be a valuable catalyst to productivity and research.

For the past thirty years, I've made it a habit to keep a mathematical journal or diary. At least when administrative or other duties haven't pulled me away from research, I try to write an entry every few days recording what I've been up to mathematically. Sometimes this consists of notes from reading, sometimes a summary of ideas from collaborators, and sometimes a record of little exercise-level statements that I've worked out. I find it especially valuable to set down questions or observations that strike me as meriting further thought. By now I've accumulated several thousand pages, originally in bound notebooks but more recently in electronically accessible form. My recollection is that I first heard about keeping a journal from Mark Goresky, although I've since come to understand that they are relatively common.

What are the benefits of mathematical journaling? We all understand that research can be frustrating, and that one can go for many weeks or months without obvious progress. But recording the various things one tries-if only to explain why they don't pan out-at least yields the satisfaction of generating concrete output on a dayto-day basis. More importantly, the very act of writing often seems to have a stimulative effect: after working on a journal entry for an hour or so, I frequently think of additional questions to ask or approaches to try. Moreover if and when the moment comes to put a problem aside, it's

Robert Lazarsfeld is Distinguished Professor of Mathematics at Stony Brook University. His email address is robert. 1azarsfeld @stonybrook.edu.

For permission to reprint this article, please contact: reprint -permission@ams.org.

DOI: http://dx.doi.org/10.1090/noti1768 valuable to have a record of one's work even if it hasn't led to a published paper.

In principle the intended audience of these journals is me alone. However when I'm involved in a project with a collaborator, sending a few journal pages is a good way to communicate. I've also found that questions I've worked on inconclusively sometimes make good research starting points for students or postdocs, and in these instances it's useful to have written notes to provide. Finally, in reflective moments it can be amusing to relive decades-old mathematical trains of thought-although like looking at baby pictures of one's grown children, one can end up feeling a bit old and nostalgic.

So-spend a few minutes to decide on a format that works for you, and then start your new mathematical journal. You'll be glad you did!

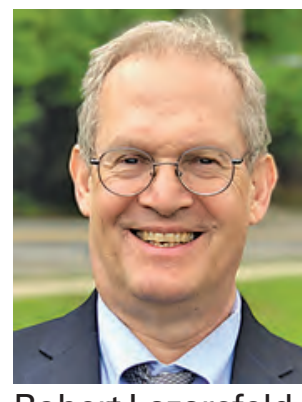

Robert Lazarsfeld

Credits

Author photo is by J. Lazarsfeld. 


\section{AMS Membership for Early Career Mathematicians}

The AMS supports early-career mathematicians with

opportunities for career development, access to information

and job listings, connections with prospective employers,

and valuable member benefits, including:

Free shipping on all purchases $\mid$ Discounts on AMS/MAA Press titles

Reduced registration at the Joint Mathematics Meetings and AMS Sectional Meetings

Free subscriptions to the Notices and Bulletin of the AMS

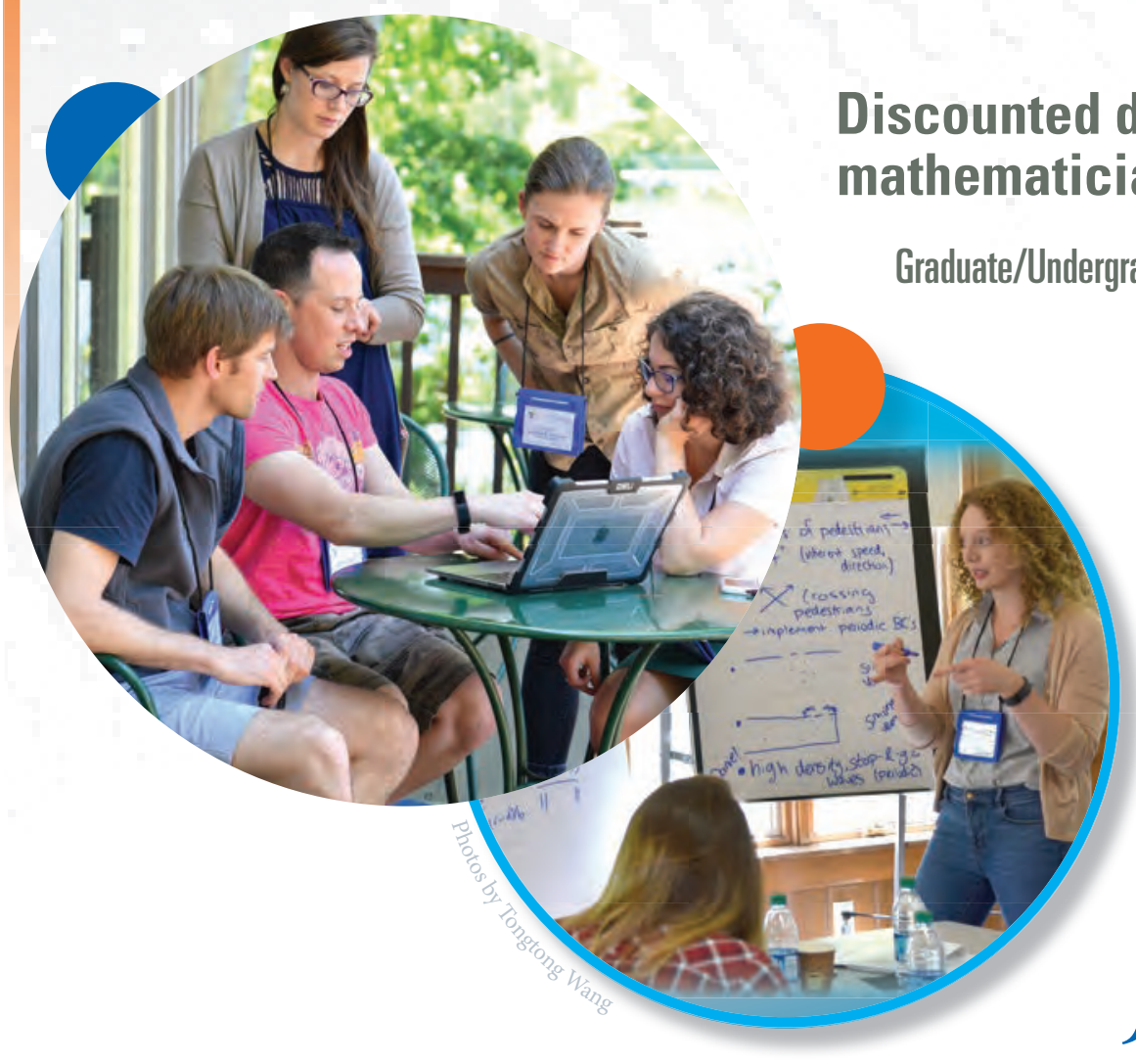

\section{Join or Renew}

your AMS membership today by visiting: www.ams.org/membership

*Applies per year to the first five consecutive years of regular membership. Eligibility begins with the first year of membership in any category other than Student, Graduate Student, or Affiliate.

\section{$\$ 50 \$ 75$}

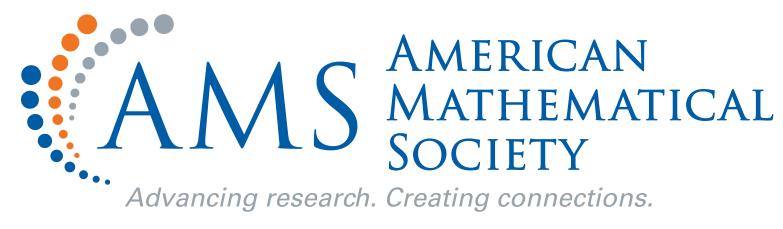




\section{Alan Baker 1939-2018}

\section{David Masser}

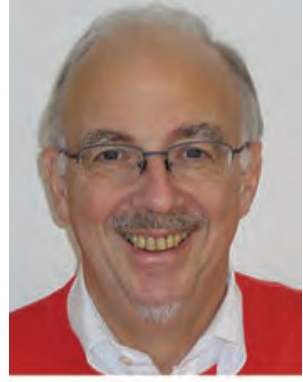

David Masser

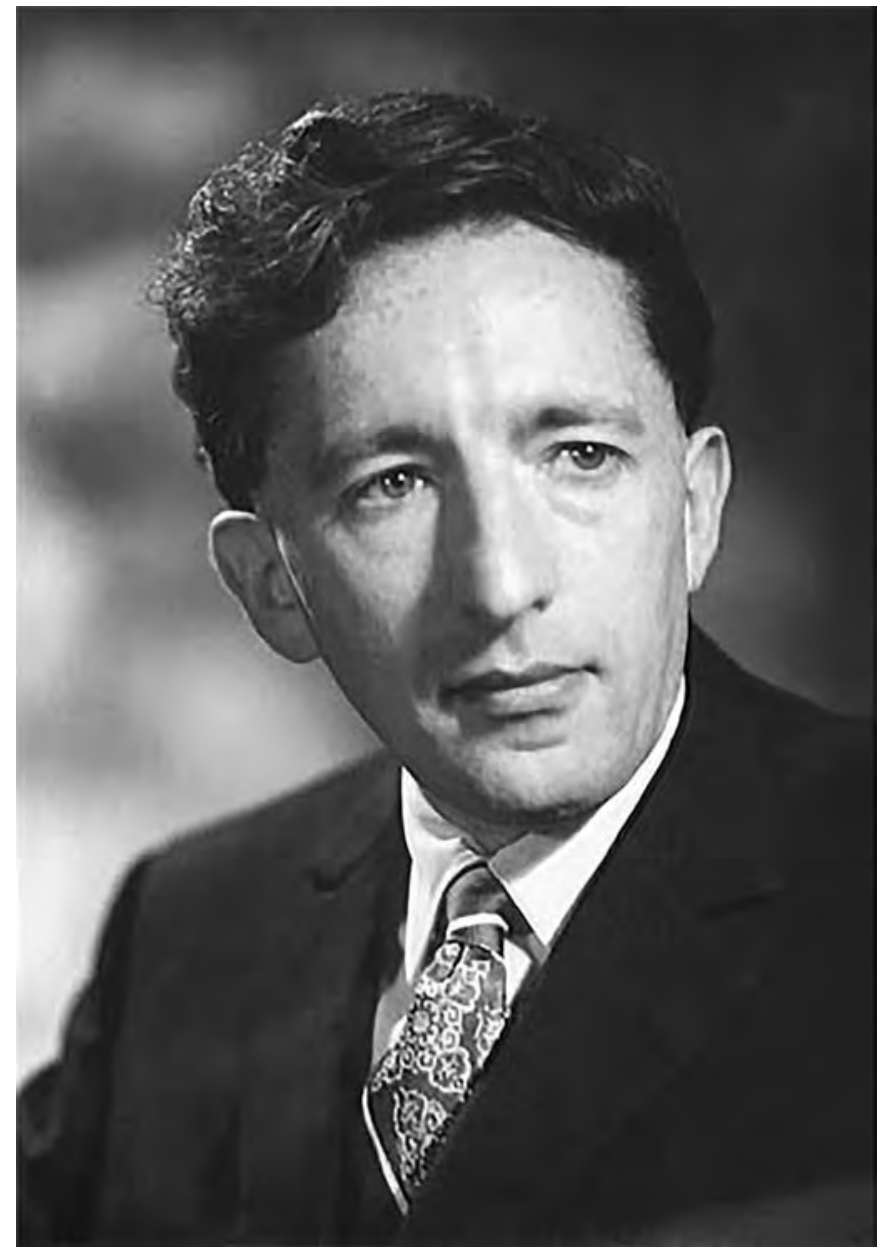

Figure 1. Alan Baker

David Masser is professor of mathematics at the University of Basel, Switzerland. His email address is david.masser@unibas.ch.

Communicated by Notices Associate Editor Della Dumbaugh.

For permission to reprint this article, please contact:

reprint-permission@ams.org.

DOI: https://doi.org/10.1090/noti/1753
Alan Baker, Fields Medallist, died on the 4th of February 2018 in Cambridge, England, after suffering a severe stroke a few days earlier.

He achieved a major breakthrough in transcendence theory and applied it to obtain a new and important large class of transcendental numbers (opening the way to the subsequent discovery of several other such classes); developed quantitative versions and applied them to the effective solutions of many classical diophantine equations as well as the resolution of the celebrated Gauss Conjecture on class numbers of imaginary quadratic number fields; and started the study of extensions to elliptic curves (opening the way to later generalizations to abelian varieties and commutative group varieties and in turn their applications to old and new problems in diophantine geometry).

The following [3] is perhaps the most easily stated of his results. Given non-zero $k$ in the ring $\mathbf{Z}$ of rational integers, all solutions $x, y$ in $\mathbf{Z}$ of the so-called "Mordell equation"

$$
y^{2}=x^{3}+k
$$

satisfy

$$
\max \{|x|,|y|\} \leq \exp \left(10^{10}|k|^{10000}\right) .
$$

Despite (1) being around since at least the year 1621, there were no estimates at all for $x, y$ until [2] in 1968. Thus given $k$ one can in principle solve (1) completely; even this fact was not previously known.

Baker's parents Barnet and Bessie (with roots in eastern Europe) lived in Forest Gate in East London, where he was born on August 19, 1939. From a very early age he showed signs of mathematical brilliance and was encouraged by his parents. Already his father was very gifted in this direction. After having attended Stratford Grammar School he went with a scholarship to University College London where he studied mathematics. He finished with a first class degree before he moved to Trinity College (where he would be based for the rest of his life) in Cambridge to study for MA and PhD degrees with Harold Davenport, one of the leading number theorists at the time with many international connections. During this time (between 1962 and 1965) he published eight papers that 
made his very high potential obvious. He received his $\mathrm{PhD}$ in 1964 and one year later was elected Fellow of Trinity in the research category. In 1970 he was awarded the Fields Medal at the International Congress in Nice on the basis of his outstanding work on linear forms in logarithms and its consequences. Since then he received many honours including the prestigious Adams Prize of Cambridge University, the election to the Royal Society (1973) and the Academia Europaea; and he was made an honorary fellow of University College London, a foreign fellow of the Indian Academy of Science, a foreign fellow of the National Academy of Sciences India, an honorary member of the Hungarian Academy of Sciences, and a fellow of the American Mathematical Society.

In 1974 he was elected to a personal chair for Pure Mathematics at the University of Cambridge. Between 1969 and 1988 he supervised a number of outstanding PhD students, and 389 mathematical descendants are listed.

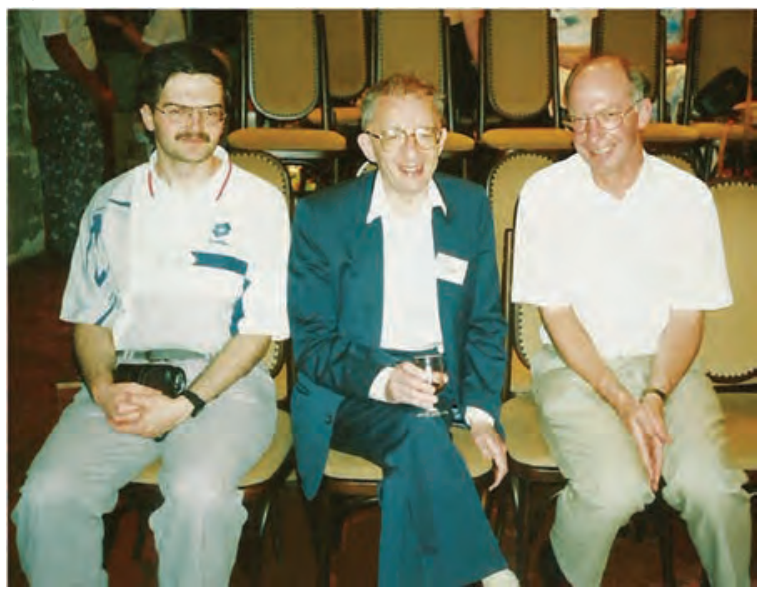

Figure 2. Mate Gyory, Alan Baker, and Rob Tijdeman at a number theory conference in Eger, Hungary, in 1996.

As mentioned, Baker had done substantial work before 1966. For example there was a very interesting article around Roth's Theorem and Mahler's Classification. But it was two papers of 1964 on rational approximation that probably made a more permanent impression on number-theorists. The second of these papers [1] established among other things the striking inequality

$$
\left|2^{1 / 3}-\frac{p}{q}\right|>\frac{10^{-6}}{q^{2.955}}
$$

for all rational integers $p, q>0$. Since Roth it had been known that the exponent 2.955 could be reduced arbitrarily close to 2, and this even for any irrational algebraic number in place of $2^{1 / 3}$; but that result (the main reason for Roth's own Fields Medal) was not "effective" in the sense that the multiplying constant $\left(10^{-6}\right.$ in the above) could not then be calculated or even estimated. And to this day no one knows how to do this, even in (3) with exponent 2.3, say.

Baker's result (3) was significant, not because 2.955 is particularly near 2 , but because it is strictly less than the degree 3 of $2^{1 / 3}$, which is the trivial exponent supplied by the much earlier ideas of Liouville. It made it an easy matter to solve completely any diophantine equation

$$
x^{3}-2 y^{3}=m
$$

in integers $x$ and $y$. Before this result, there had been no algorithm at all, just as for (1).

Strangely enough, Baker's proof (using the so-called Padé theory with an extra 3-adic twist) broke down completely for

$$
x^{3}-5 y^{3}=m .
$$

He himself found a way round this, and in the process opened up an entirely new area of diophantine approximation, with his wonderful sequence [2] of papers on linear forms in logarithms dating from 1966.

The classical theorem of Hermite-Lindemann is equivalent to the fact that if $\alpha$ is a non-zero algebraic number, and $\log \alpha$ is any non-zero choice of its complex logarithm, then 1 and $\log \alpha$ are linearly independent over the field $\overline{\mathbf{Q}}$ of all algebraic numbers. Similarly the classical theorem of Gelfond-Schneider is equivalent to the fact that if $\alpha_{1}, \alpha_{2}$ are non-zero algebraic numbers, and $\log \alpha_{1}, \log \alpha_{2}$ are any choices of logarithms that are linearly independent over the field $\mathbf{Q}$ of rational numbers, then they are linearly independent over $\overline{\mathbf{Q}}$. Nothing was known about even three logarithms until Baker proved (1966-68)

Theorem. If $\alpha_{1}, \ldots, \alpha_{n}$ are non-zero algebraic numbers, and $\log \alpha_{1}, \ldots, \log \alpha_{n}$ are any choices of logarithms which are linearly independent over $\mathbf{Q}$, then

$$
1, \log \alpha_{1}, \ldots, \log \alpha_{n}
$$

are linearly independent over $\overline{\mathbf{Q}}$.

The reader may easily construct simple examples of transcendental numbers not covered by Hermite-Lindemann or Gelfond-Schneider; a less simple example is

$$
\int_{0}^{1} \frac{\mathrm{d} x}{x^{3}+1}=\frac{\pi \sqrt{3}}{9}+\frac{\log 2}{3}
$$

quoted by Siegel in his famous transcendence monograph.

In a paragon of modesty, clarity, and foresight, Baker wrote in the first of his sequence

Finally, as regards the proof of the theorem, our method depends on the construction of an auxiliary function of several complex variables which would seem to be the natural generalisation of the function of a single variable used in Gelfond's original work. The subsequent treatment employed by Gelfond, 


\section{MEMORIAL TRIBUTE}

however, is not applicable in the more general context and so it has been necessary to devise a new technique. Nevertheless it will be appreciated that the argument involves many familiar ideas. The method will probably be capable of considerable development for it applies in principle to many other auxiliary functions apart from the one constructed here.

Let us give an idea of this "new technique," for simplicity taking $n=3$ and ignoring the extra 1 in (5), so that we have to deduce a contradiction from a relation

$$
\beta_{1} \log \alpha_{1}+\beta_{2} \log \alpha_{2}=\log \alpha_{3}
$$

with $\beta_{1}, \beta_{2}$ also algebraic. The auxiliary function $\Phi\left(z_{1}, z_{2}\right)$ is a polynomial of large degree in

$$
e^{Z_{1}}, e^{Z_{2}}, e^{\beta_{1} Z_{1}+\beta_{2} Z_{2}}
$$

which indeed generalize in a fairly natural way (although no one had previously written them down) Gelfond's $\Phi(z)$ and $e^{z}, e^{\beta z}$. Note that from (6) the functions (7) take algebraic values at all points

$$
\left(Z_{1}, z_{2}\right)=\left(s \log \alpha_{1}, s \log \alpha_{2}\right), s=0,1,2, \ldots
$$

and this is true even of their partial derivatives. That enables $\Phi$ to be constructed, with algebraic coefficients not all zero, such that

$$
\frac{\partial^{t_{1}}}{\partial z_{1}^{t_{1}}} \frac{\partial^{t_{2}}}{\partial z_{2}^{t_{2}}} \Phi\left(s \log \alpha_{1}, s \log \alpha_{2}\right)=0
$$

for all non-negative integers $s, t_{1}, t_{2}$ in some large range

$$
s \leq S, t_{1}+t_{2} \leq T .
$$

Gelfond had shown by an extrapolation technique on his $\Phi(z)$ that his range $s \leq S, t \leq T$ could be extended to say $s \leq S, t \leq 2 T$. This step could be then iterated, even indefinitely, to get zeroes of infinite multiplicity and so the required contradiction.

To this day no one knows how to increase $T$ in (8) to $2 T$. But Baker, by applying similar extrapolation techniques on all the separate $\Phi_{\tau_{1}, \tau_{2}}(z)=\frac{\partial^{\tau_{1}}}{\partial z_{1}^{\tau_{1}}} \frac{\partial^{\tau_{2}}}{\partial z_{2}^{\tau_{2}}} \Phi\left(z \log \alpha_{1}, z \log \alpha_{2}\right)$, $\tau_{1}+\tau_{2} \leq T / 2$ was able to modify (8) to say

$$
s \leq 8 S, t_{1}+t_{2} \leq T / 2 .
$$

The number of conditions here is roughly twice that in (8) and so we have gained something. We can iterate but not indefinitely in any profitable way. Already this was a new sort of difficulty, which Baker overcame by getting just as many zeroes as are needed for the contradiction (along the principle that a polynomial of degree $D$ cannot have $D+1$ zeroes).

So Baker's main achievement was to introduce several complex variables (not being afraid of possible Hartogsstyle complications), reduce them to a single variable along a line, and supply the missing zero estimates.

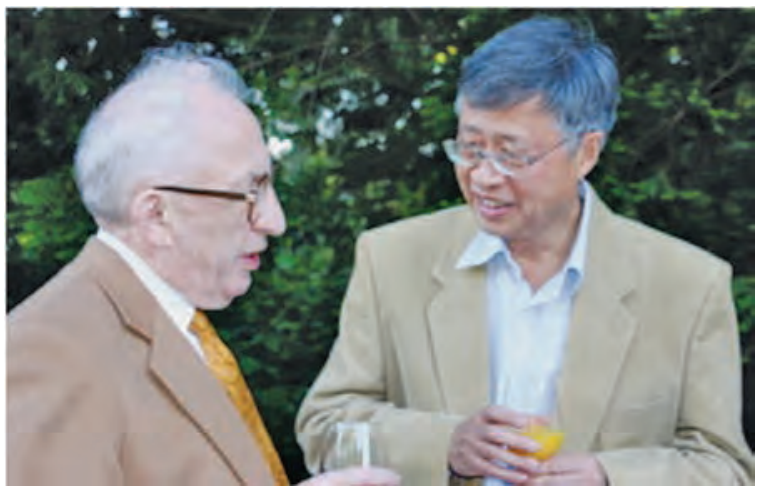

Figure 3. Alan Baker with Yu Kunrui on the occasion of Peter Sarnak's 61st birthday conference at the IAS in 2014.

In fact he did more, obtaining positive lower bounds for the absolute value of linear forms

$$
\beta_{0}+\beta_{1} \log \alpha_{1}+\cdots+\beta_{n} \log \alpha_{n}
$$

in terms only of certain complexity measures (heights) of the algebraic numbers appearing. This was vital for the applications; and crucial too was that the lower bounds should be sufficiently strong. The earlier bounds sufficed for (1) and (4), but more sophistication was needed for his solution $[2, \mathrm{I}]$ of the Gauss Conjecture that the only imaginary quadratic fields with class number $h=1$ have discriminant $\Delta$ at most 163 in absolute value, and even more for his extension [5] to $h=2$ (there followed a collaboration with Stark who had independently obtained these results) leading finally to $|\Delta| \leq 427$. Similarly the work led to Feldman's improvement on the Liouville exponent for any algebraic number of degree at least three, for example

$$
\left|5^{1 / 3}-\frac{p}{q}\right|>\frac{10^{-12900}}{q^{2.9999999999998}}
$$

(also by which (4) can be solved) due to Baker and Stewart [8]. And with Wüstholz [9] in 1993 Baker took the lower bounds for (9) already extremely close to their modernday versions.

The foresight in the above quotation was illustrated by Baker himself in making a start [4] on analogues of his Theorem for elliptic functions, where $\log \alpha=\int_{1}^{\alpha} \mathrm{d} x / x$ is replaced by an "elliptic logarithm"

$$
\int_{\infty}^{\alpha} \frac{\mathrm{d} x}{\sqrt{4 x^{3}-g_{2} x-g_{3}}} .
$$

He gave an account of much of this (and more besides) in his book [6], a worthy successor to the classics of Siegel, Gelfond, and Schneider.

Baker single-handedly transformed the subject of transcendence and diophantine approximation, and others have 
taken things yet further. For example Tijdeman used linear forms in logarithms to show that there are at most finitely many solutions $p>1, q>1, r>1, s>1$ to Catalan's equation $p^{s}-q^{r}=1$, and then Mihăilescu showed that the only solution is indeed $(p, q, r, s)=(3,2,3,2)$ as Catalan had conjectured. And various authors developed the elliptic and higher analogues culminating in the work of Wüstholz on general commutative group varieties. This further led via so-called "isogeny estimates" to effective versions of Faltings's Finiteness Theorems and the Tate Conjecture for abelian varieties, and even to the solution of geometric problems such as the existence of "small" polarizations. Some of this was in turn described in Baker's book [10] (not to be confused with the attractive [7], much more elementary) with Wüstholz. Since then the material has been found useful also in aspects of the André-Oort Conjecture.

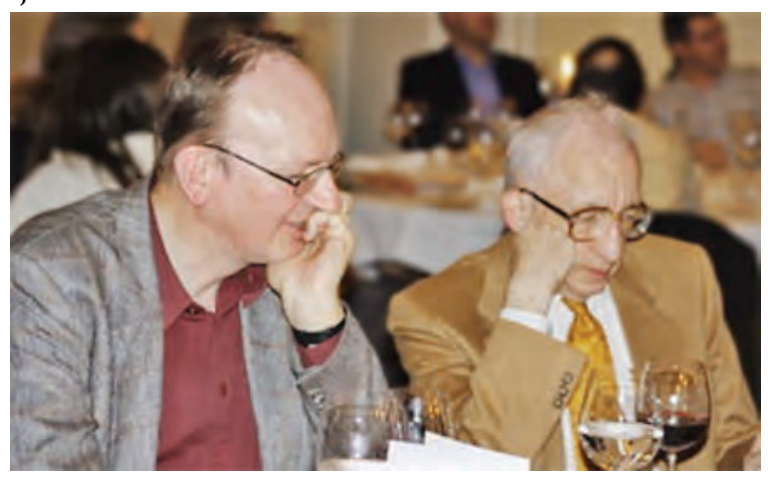

Figure 4. Gerd Faltings and Alan Baker on the occasion of Peter Sarnak's 61st birthday conference at the IAS in 2014.

As mentioned, Baker was firmly based in Cambridge; it seems that college life there suited him especially in the style of Trinity, whose society he enriched. He had a flat in London and enjoyed life there too, for example the theatre. He was enthusiastic about travel, and as his reputation grew he was able to combine this with professional visits to China and many parts of Europe and especially of America. In later life he made regular trips to Switzerland to work with Wüstholz at ETH Zürich. It was there, during a conference in honour of his 60th birthday, that he gave an entertaining and typically candid speech about his life, starting with his recollections of wartime London and ending with his regrets about never marrying.

\section{References}

[1] Baker A. Rational approximations to $\sqrt[3]{2}$ and other algebraic numbers, Quart. J. Math. Oxford 15 (1964), 375-383. MR0171750
[2] Baker A. Linear forms in the logarithms of algebraic numbers I,II,III,IV, Mathematika 13 (1966), 204-216; 14 (1967), 102-107, 220-228; 15 (1968), 204-216. MR0220680

[3] Baker A. Contributions to the theory of Diophantine equations II - the Diophantine equation $y^{2}=x^{3}+$ $k$, Phil. Trans. Royal Soc. London A263 (1968), 193-208. MR0228425

[4] Baker A. On the periods of the Weierstrass $\wp$-function, Symposia Math. IV, INDAM Rome 1968, Academic Press, London 1970, pp.155-174. MR0279042

[5] Baker A. Imaginary quadratic fields with class number 2, Annals of Math. 94 (1971), 139-152. MR0299583

[6] Baker A, Transcendental Number Theory, Cambridge University Press 1975 (and third edition 1990). MR0422171

[7] Baker A. A Concise Introduction to the Theory of Numbers, Cambridge University Press 1984. MR0781734

[8] Baker A. Stewart CL, On effective approximations to cubic irrationals, in New Advances in Transcendence Theory (ed. A. Baker), Cambridge University Press 1988, pp.1-24. MR0971990

[9] Baker A. Wüstholz G, Logarithmic forms and group varieties, J. reine angew. Math. 442 (1993), 19-62. MR1234835

[10] Baker A. Wüstholz G, Logarithmic Forms and Diophantine Geometry, Cambridge University Press 2007. MR2382891

\section{Credits}

Figure 1 is Fair Use. The authors and editors have made every reasonable effort to identify the copyright holder of this photo.

Figure 2 is courtesy of Kálmán Györy.

Figures 3 and 4 are courtesy of Gisbert Wüstholz. Author photo is courtesy of the author. 
MATHSCINET ${ }^{\oplus}$ FOR DEVELOPING COUNTRIES JMM CHILD CARE GRANTS

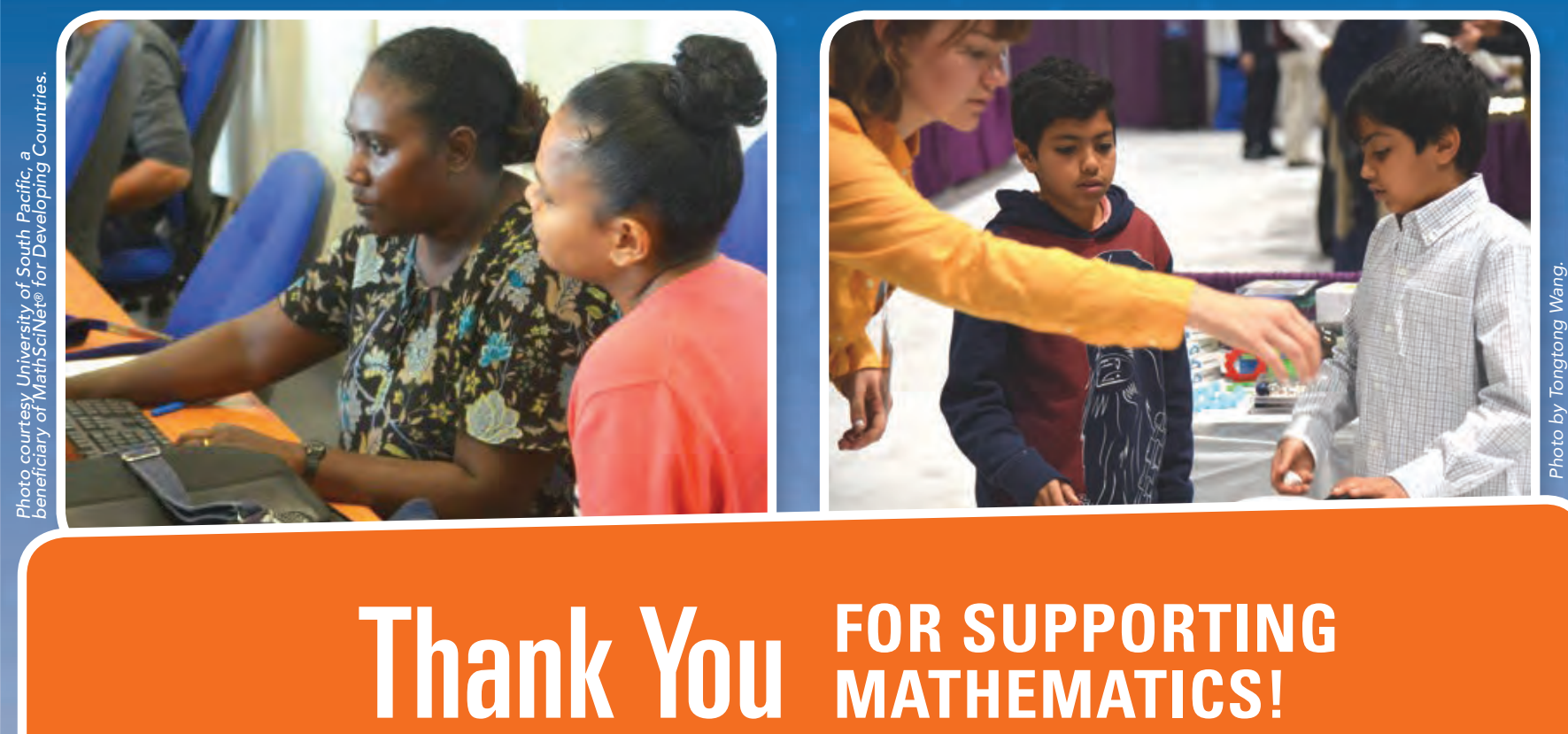

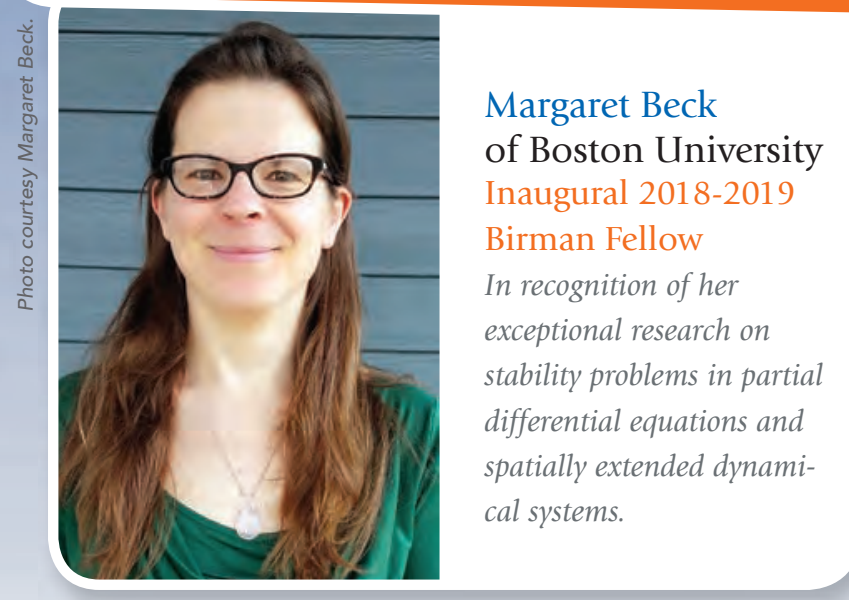

JOAN \& JOSEPH BIRMAN FELLOWSHIP FOR WOMEN SCHOLARS

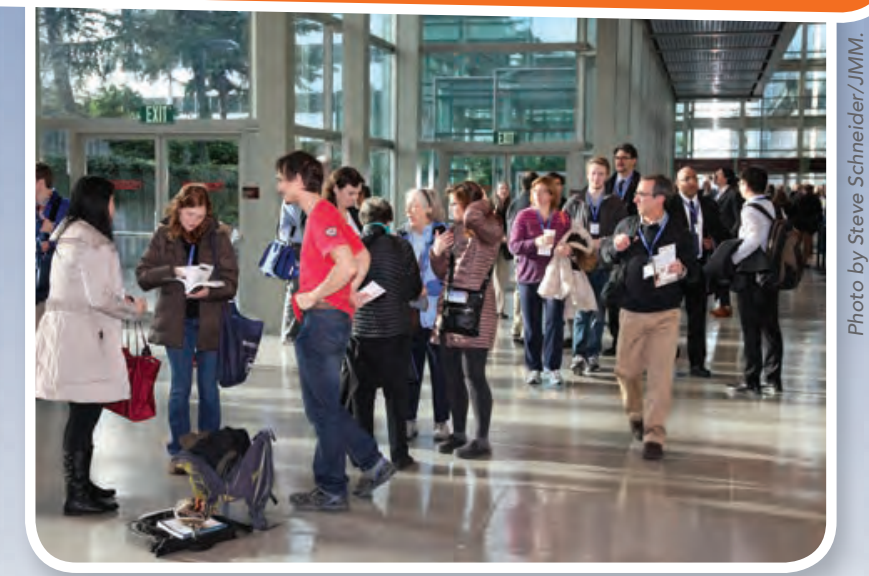

AREA OF GREATEST NEED:

MATHEMATICS MEETINGS | GOVERNMENT AND POLICY ADVOCACY FOR MATHEMATICS | EMPLOYMENT SERVICES PUBLIC AWARENESS AND OUTREACH | \& MUCH MORE

TO LEARN MORE ABOUT WHAT YOUR GIFT ACCOMPLISHES, VISIT WWW.AMS.ORG/SUPPORT 


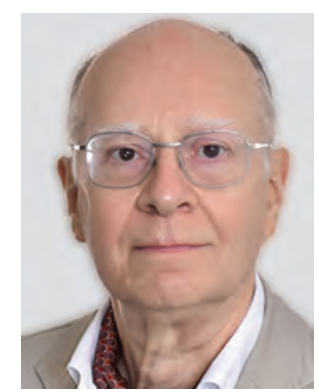

Luc Illusie

\section{Michel Raynaud (1938-2018) Luc Illusie}

Michel Raynaud's parents lived in Châtel-Guyon, a small spa town in the department of Puy-de-Dôme, located in the mountainous Auvergne region in the center of France. His father was a carpenter, and his mother, a cleaning lady. He was born on June 16, 1938, in Riom, a bigger town five miles to the southeast. He was their only child. He attended elementary school in Châtel-Guyon, then middle school in Riom, and, as a boarding student, high school in Clermont-Ferrand, the main city of the department. He entered the École Normale Supérieure (Paris) in 1958. I myself was admitted in 1959 and it is there that we met for the first time. I remember that he impressed me by his understanding of Lang's book on abelian varieties and Grothendieck's newly published first volumes of [EGA]. That was the beginning of a friendship that lasted until his death.

In 1961 he was number one on the "agrégation" exam, the national competitive examination selecting high school teachers in France. After one more year at the École Normale, he was admitted at the CNRS (Centre National de la Recherche Scientifique), where he stayed until 1967. Meanwhile he had met Michèle Chaumartin, who had entered the École Normale Supérieure de Jeunes Filles in 1958, and whom he married in 1962. They had a son, Alain, born in 1970, who now lives in California with his wife and two daughters.

In 1962, Michel and Michèle started attending Serre's course at the Collège de France and Grothendieck's algebraic geometry seminar [SGA] at the IHÉS. Grothendieck

Luc Illusie is a retired professor of mathematics at Université Paris-Sud. His email address is Luc. I11usie@math.u-psud. fr.

Communicated by Notices Associate Editor Daniel Krashen.

For permission to reprint this article, please contact: reprint - permission@ams .org.

DOI: http://dx.doi.org/10.1090/noti1764 became their PhD advisor, and they contributed to his seminars ([SGA 3, 6] for Michel, and [SGA 1, 2, 7] for Michèle). After rejecting various suggestions of Grothendieck, Michel chose his own topic for his thesis, and defended it in 1968 [R70a]. In Récoltes et semailles, Grothendieck wrote: "Michel Raynaud prend une place à part, ayant trouvé par lui-même les questions et notions essentielles qui font l'objet de son travail de thèse, qu'il a de plus développé de façon entièrement indépendante; mon rôle de 'directeur de thèse' proprement dit s'est donc borné à lire la thèse terminée, à constituer le jury et à en faire partie.."1 He was hired as a professor at Orsay in 1967 and worked there until his retirement in 2001.

Raynaud was a modest person. He disliked honors, but several were nevertheless bestowed on him. He was an invited speaker at the Nice ICM in 1970 [R71]. In 1987 he was awarded the Ampère Prize of the French Academy of Sciences. He was elected corresponding member of the French Academy of Sciences in 1994. In 1995 he received the Frank Nelson Cole Prize, jointly with David Harbater, for the proof of the Abhyankar conjecture. On the occasion of his retirement a conference Algebraic Geometry and Applications to Number Theory, June 18-22, 2001, was held at Orsay in his honor.

Raynaud was the emblematic figure of algebraic geometry in our department at Orsay. In 1976 he founded the Équipe d'arithmétique et géométrie algébrique, a research unit associated with the CNRS, of which he was the head until 1983. He then let me succeed him. Together we founded the SAGA (Séminaire d'arithmétique et géométrie

\footnotetext{
${ }^{1}$ Michel Raynaud occupies a special place, as he found by himself the essential notions and questions that are the subject of his thesis, and which he developed in a totally independent way; my role of 'thesis advisor' was thus limited to reading the completed thesis, selecting the jury, and participating in it.
} 
algébrique) in 1985. It quickly became popular, and is still very active.

Every morning, he arrived at his office at 7:00 am. Many people came to consult him, sometimes from far away. Teaching mattered very much to him, at all levels. Models of clarity, his courses have inspired generations of students. He directed ten PhD theses. His students remember his demand for rigor, his generosity, and his kindness. For many years he chaired the committee in charge of the teaching assignments. Together with Guy Henniart he developed a successful teacher preparation (and pre-preparation) program for students planning to take the agrégation, in which his former students Lucile Bégueri and Renée Elkik, who had become his colleagues at Orsay, actively participated.

Raynaud was quite influential in the development of our ties with Japan and China. His Chinese student Xiao Gang, who died prematurely in 2014, created a school of complex algebraic geometry in Shanghai. In 1982, together with Tetsuji Shioda, he organized a Japan-France conference Algebraic Geometry [RS83] at Tokyo and Kyoto that was the starting point of a sustained and fruitful collaboration between French and Japanese algebraic geometers. He visited Japan several times. He also visited China once, in the spring of 2004: in the framework of a cooperative program supervised by Jean-Marc Fontaine, and kindly assisted by our Chinese colleague Yi Ouyang, Raynaud and I gave a course at Tsinghua University. At the end of it we selected students

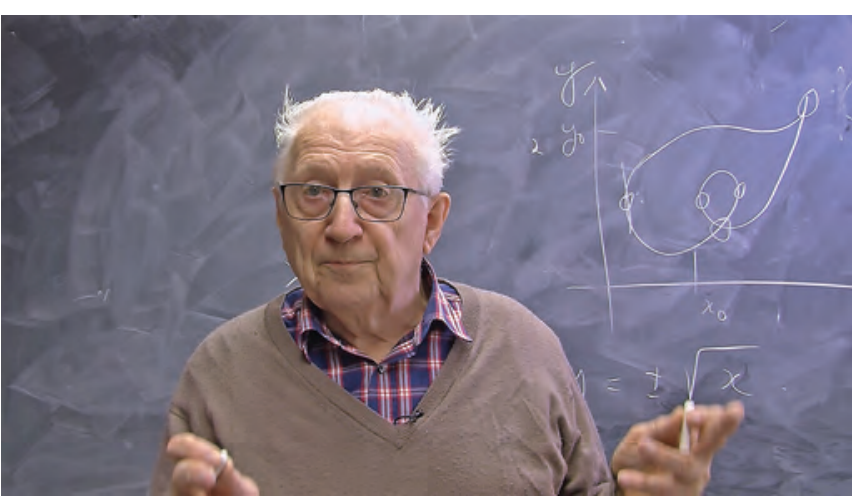

Michel Raynaud lecturing.
(2) The Abhyankar conjecture. For the affine line $X$ over an algebraically closed field $k$ of characteristic $p>0$, this conjecture asserts that any finite group $G$ which is generated by its $p$-Sylow subgroups is the Galois group of a connected étale Galois cover of X. Raynaud proved it in [R94a], again by rigid geometry techniques (based on the work described in (3) below). The problem had been proposed to him by Serre, who had treated the case where $G$ is solvable. Shortly afterwards, Harbater [H94], elaborating on Raynaud's work, proved the general case of the Abhyankar conjecture (for affine smooth curves over $k$ ).

(3) A new approach to rigid geometry. Rigid analytic spaces over complete non-archimedean valued fields $K$ were defined by Tate in 1961 and studied by him and, a few years later, by Kiehl. In the early 1970s Raynaud [R74] introduced a new way of thinking about them, by means of a category of formal models where certain blow-ups, called "admissible," are inverted: a typical example is his construction of the rigid generic fiber of a (flat, finitely presented) formal scheme over the ring of integers of $K$. The dictionary he built between formal and rigid geometry enabled him to recover Tate's and Kiehl's results by a simple application of techniques of [EGA]. He used it in [R71] to construct rigid uniformizations of abelian schemes over a complete discrete valuation field $K$ having semi-abelian reduction (generalizing Tate's construction of the Tate curve), and, later, similar uniformizations for 1 -motives over $K$ in the sense to come to Orsay to pursue a PhD (Raynaud's last student, Jilong Tong, was one of them). More came afterwards, and a good number are now university professors in China. This 2004 exchange with China led to many others in later years.

Raynaud's work is vast and touches several different topics. I will limit myself to brief comments on a few salient points.

(1) The Manin-Mumford conjecture. Raynaud proved the following generalization of this conjecture: let $A$ be an abelian variety over an algebraically closed field $k$ of characteristic zero, and let $X$ be a closed integral subscheme of $A$. Denote by $T$ the torsion subgroup of $A(k)$. If $T \cap X(k)$ is Zariski dense in $X$, then $X$ is the translate of an abelian subvariety of $A .^{2}$ Raynaud first treated the case where $X$ is a curve [R83a], by reduction modulo $p^{2}$ techniques, then the general case [R83b] using additional results from his approach (see (3) below) to rigid geometry.

\footnotetext{
${ }^{2}$ The Manin-Mumford conjecture was for the case of a curve embedded in its Jacobian.
}

of Deligne [R94b]. This viewpoint, which is crucial there and in (1) and (2), has been very influential, e. g. in crystalline cohomology (Berthelot's rigid cohomology) and p-adic Hodge theory (Faltings' almost purity theorem and applications).

(4) Flatness. Grothendieck showed the importance of flatness in algebraic geometry: the good families of algebraic varieties are those which correspond to a flat morphism of schemes $f$. Such morphisms, often with the extra condition that $f$ be locally of finite presentation, were extensively studied in [EGA IV]. However, for the foundations of his new approach to rigid geometry, Raynaud needed more. In a seminal joint paper with Gruson [RG71] he proved that, given a morphism $f: X \rightarrow S$ satisfying mild assumptions ( $S$ quasi-compact and quasi-separated, and $f$ of finite presentation), there exists a blow-up g: $S^{\prime} \rightarrow S$ such that the strict transform $f^{\prime}: X^{\prime} \rightarrow S^{\prime}$ of $f$ is flat (and if $f$ is flat over some open $U$ in $S$, then g can be chosen to be an isomorphism over $U$ ). This flattening theorem was critical for 
rigid geometry but has had many other applications (the above paper is Raynaud's most cited paper).

(5) The Picard functor and Néron models. The main part of this work was done in the 1960s. It reflects Raynaud's early interest for abelian varieties, and was partly influenced by Grothendieck, who, among other things, had proposed to him translating Néron's construction of Néron models into the language of schemes. In the late 1950s Grothendieck had proved the representability (by a scheme) of the relative Picard functor $\mathrm{Pic}_{X / S}$ for projective, flat morphisms $X \rightarrow S$ with integral geometric fibers. In [R70b] Raynaud studies the case where $S$ is a trait (spectrum of a discrete valuation ring), but where the geometric fibers are no longer assumed to be integral. In particular, for $X / S$ a proper, flat curve, with smooth, geometrically irreducible generic fiber, he gives practical conditions ensuring that $\mathrm{Pic}_{X / S}^{0}$ is the connected component of the Néron model $A$ of the Jacobian of the generic fiber of $X$, and provides a combinatorial description of the group of connected components of the special fiber of $A$ in terms of the irreducible components of the special fiber of $X$. These results had important applications: they were of critical use in Deligne-Mumford's article [DM69], and later in the analysis of the arithmetic of the modular curve $X_{0}(N)$ in work of Deligne-Mazur-Rapoport, ${ }^{3}$ and Ribet (in his proof that the Shimura-Taniyama-Weil conjecture implies Fermat [R90]). An extended presentation of his results, together with useful pedagogical material, is given in a beautiful book coauthored with Bosch and Lütkebohmert [BLR90].

(6) Group schemes of type $(p, \cdots, p)$. Raynaud brought several contributions to the theory of group schemes: in [SGA 3] (Exp. XV, XVI, XVII), and in his thesis [R70a], but certainly the best known one is his paper [R74b] on group schemes of type $(p, \cdots, p)$, i.e., finite, flat, commutative group schemes annihilated by a prime number $p$. Let $R$ be a strictly henselian discrete valuation ring of mixed characteristic $(0, p)$, absolute ramification index $e$, fraction field $K$, and let $\bar{K}$ be an algebraic closure of $K$ and $I=\mathrm{Gal}(\bar{K} / K)$ the inertia group. Raynaud's paper [R74b] contains two independent main results:

(a) For $e \leq p-1$, if $G$ is a group scheme of type $(p, \cdots, p)$ over $R$, then any Jordan-Hölder quotient $H$ of $G$ is a group scheme in vector spaces over a field $\mathbf{F}_{p}{ }^{r}$, the inertia $I$ acts

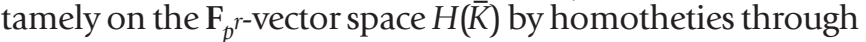
a character $\mathbf{F}_{p^{r}}^{*} \rightarrow \mathbf{F}_{p^{r}}^{*}$ which is a product of fundamental characters with exponents $\leq e$. This proves a conjecture of Serre.

\footnotetext{
$\overline{3 \text { In particular, for the modular curve } X_{0}(p), p \geq 5 \text {, the group of connected }}$ components of the special fiber at $p$ of the Néron model of the Jacobian of $X_{0}(p)_{\mathbf{Q}}$ is, over an algebraic closure of $\mathbf{F}_{p^{\prime}}$ a cyclic group of order the numerator of $(p-1) / 12$ [M77, Appendix].
}

(b) If $G$ is a $p$-divisible group over $R$, of height $h$ and dimension $d$, with Tate module $T=T_{p}\left(G_{K}\right)$, then $\wedge^{h} T=Z_{p}$ $(d)$, where $Z_{p}(d)=T_{p}\left(\left(\mu_{p}^{\infty}\right)_{K}\right)^{\otimes d}$.

These results have been very fruitful; for example, (b) was used by Faltings [F84] in his proof of the Mordell conjecture to bound the modular height in an isogeny class of abelian varieties (effective refinements were given by Raynaud in the Szpiro seminar [R85]).

(7) Cartier-Dieudonné theory and crystalline cohomology. In his proof of (6)(b) Raynaud used Cartier's theory of formal groups. He returned to it-and to the Picard functor-in [R79], where he made a thorough study of the proper $R$-scheme $P \mathrm{Pic}_{X / R}^{\tau}$ for $R$ as in $(6)^{4}$ and X/R proper and smooth. He then got interested in crystalline cohomology and the de Rham-Witt complex of proper, smooth schemes over a perfect field of characteristic $p>0$. It was known in the late 1970s that the initial term of the so-called slope spectral sequence associated with this complex gave, modulo $p$-torsion, formal $p$-divisible groups, but the structure of its $p$-torsion remained mysterious. We unraveled it in [IR83] in terms of certain graded modules over a graded ring, now called the Raynaud ring, which is an extension of the classical Cartier-Dieudonné ring. Raynaud, who often jokingly boasted of not understanding spectral sequences, in fact discovered the deepest property of the slope spectral sequence (and of its partner, the conjugate spectral sequence), that he suggested to call the survival of the heart. Despite its romantic name, this property is unfortunately too technical to be described here. This theory was extensively developed by Ekedahl [E84-86]. ${ }^{5}$ Other geometric and arithmetic applications were found later by Joshi and Milne-Ramachandran.

(8) Geometry of curves and surfaces. Raynaud [R78] gave the first example of a proper, smooth surface $X$ over an algebraically closed field $k$ of characteristic $p>0$ having an ample line bundle $\mathcal{L}$ such that $H^{1}\left(X, \mathcal{L}^{-1}\right) \neq 0$, thus showing that the Kodaira vanishing theorem does not extend to positive characteristic. ${ }^{6}$ His construction is based on certain curves studied by Tango. Relative variants were developed jointly with Szpiro and new examples were given. These curves are now called Tango-Raynaud curves.

In the mid 1970s Raynaud studied elliptic and quasi-elliptic fibrations of surfaces. His work remained unpublished but notes circulated. It was of essential use in joint papers with Liu and Lorenzini ([LLR04], [LLR05]) where they prove the following. Let $X$ be a proper, smooth, and geometrically connected surface over a finite field $k$, $f: X \rightarrow V$ a proper and flat morphism to a proper, smooth,

\footnotetext{
${ }^{4}$ (supposed further to be complete, and have an algebraically closed residue field)

${ }^{5}$ See [RS83, 20-72] for a survey.

${ }^{6}$ Mumford had previously given an example with a singular, normal surface
} 
geometrically connected $k$-curve with smooth, geometrically connected generic fiber $X_{K}$. Then: (a) (under a certain necessary hypothesis on local numerical invariants of $f$ ) the Artin-Tate and Birch and Swinnerton-Dyer conjectures are equivalent; (b) if, for some prime $\ell$, the $\ell$-part of the Brauer group $\operatorname{Br}(X)$ is finite, then the order of $\operatorname{Br}(X)$ is a square, which resolved a longstanding question.

Let now $X$ be a proper, smooth, connected curve of genus $g \geq 1$ over an algebraically closed field $k$ of characteristic $p>0$, with relative Frobenius $F: X \rightarrow X^{(p)}$. The sheaf $B \subset F_{*} \Omega_{X / k}^{1}$ of locally exact differentials, a vector bundle of rank $p-1$ and slope $g-1$ over $X^{(p)}$, appeared in the definition of Tango-Raynaud curves. Raynaud [R82] showed that $B$ admits a theta divisor $D \subset J^{(p)}$ (where $J$ is the Jacobian of $X$ ) parametrizing invertible sheaves $L$ of degree zero such that $h^{0}(B \otimes L) \neq 0$. This divisor $D$ turned out to be a key ingredient in the proof by Tamagawa [T04] of the fact that, over an algebraic closure of $\mathbf{F}_{p}$ there are only finitely many isomorphism classes of smooth, hyperbolic curves whose tame fundamental group is isomorphic to a given profinite group.

(9) Bourbaki seminar. Raynaud gave several talks in the Bourbaki seminar, on a wide range of topics. See the references at the end.

Let me finish on a more personal tone. Raynaud was fond of nature. His office at Orsay overlooked the woods. From time to time he would watch a red squirrel bouncing in the trees. He kept an amaryllis on his desk. In their youth he and Michèle climbed in the Alps, mostly in the Oisans massif. For almost forty years they went skiing each year in Val d'Isère. Starting in the late 1960s, together with Serre they regularly did rock climbing in Fontainebleau. Michel and Michèle also used to play tennis three times a week. He remained in rather good health until one day, in November 2017, he suddenly became disoriented near his house in Palaiseau. An MRI revealed a brain tumor. Most of it was successfully removed by surgery shortly before Christmas, and for a few weeks he seemed to have recovered. However, in February his condition deteriorated rapidly. He had to be hospitalized, he fell into coma, and he died on March 10.

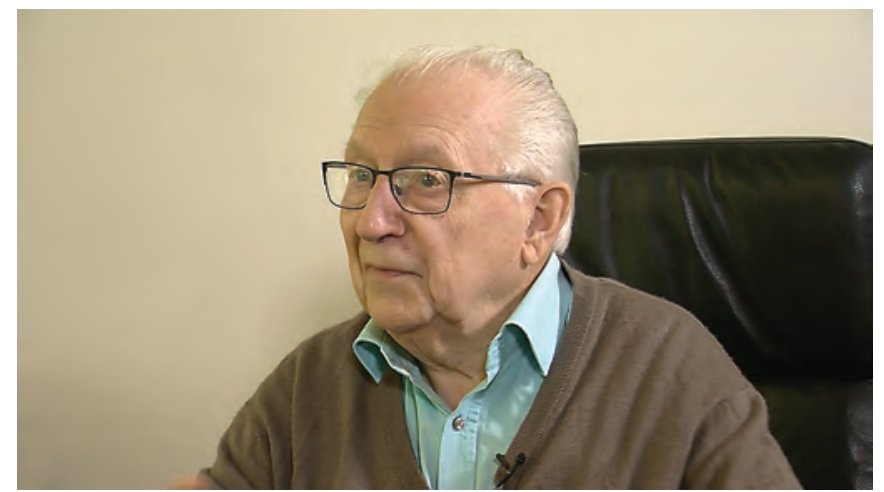

Interview with Michel Raynaud.
Up to his last moments of consciousness he remained mentally alert and kept his sharp sense of humor. When I visited him at the hospital, we joked again about spectral sequences, of which he said that they had luckily disappeared in the surgery, and also about rigid annuli, which he loved, especially those of thickness zero, which had played a key role in his proof of the Abhyankar conjecture.

ACKNOWLEDGMENTS. I thank Lucile Bégueri, Renée Elkik, Jean-Marc Fontaine, Nick Katz, Michèle Raynaud, and Jean-Pierre Serre for helpful remarks on a first draft of this text.

\section{References}

[R70a] Faisceaux amples sur les schémas en groupes et les espaces homogènes. Lecture Notes in Math. 119, SpringerVerlag, Berlin, 1970. MR0260758

[R70b] Spécialisation du foncteur de Picard. Inst. Hautes Études Sci. Publ. Math. No. 38 (1970), 27-76. MR0282993

[R71] Variétés abéliennes et géométrie rigide. Actes du Congrès International des Mathématiciens (Nice, 1970), Tome 1, 473-477. Gauthier-Villars, Paris, 1971. MR0427326

[RG71] Raynaud, M, Gruson, L, Critères de platitude et de projectivité, Invent. Math. 13 (1971), 1-89. MR0308104

[R74a] Géométrie analytique rigide d'après Tate, Kiehl,... Table ronde d'analyse non archimédienne (Paris, 1972), 319-327. Bull. Soc. Math. France, Mém. 39-40, Soc. Math. France, Paris, 1974. MR0470254

[R74b] Schémas en groupes de type (p,..., p). Bull. Soc. Math . France 102 (1974), 241-280. MR0419467

[R78] Contre-exemple au "vanishing theorem" en caractéristique $p>0$. Ramanujam, CP-a tribute, pp. 273-278, Tata Inst. Fund. Res. Studies in Math., 8, Springer-Verlag, Berlin, 1978.MR0541027

[R79] "p-torsion" du schéma de Picard, Journées de géométrie algébrique de Rennes (Juillet 1978) II, Astérisque 64 (1979), 87-148. MR0563468

[R82] Sections des fibrés vectoriels sur une courbe. Bull. Soc. Math. France 110 (1982), no. 1, 103-125. MR0662131

[R83a] Courbes sur une variété abélienne et points de torsion. Invent. Math. 71 (1983), 207-283. MR0688265

[R83b] Sous-variétés d'une variété abélienne et points de torsion. Arithmetic and Geometry, Vol. I, 327-352, Progr. Math. 35, Birkhäuser Boston, Boston, MA, 1983. MR0717600

[IR83] Illusie, L, Raynaud, M, Les suites spectrales associées au complexe de de Rham-Witt. Inst. Hautes Études Sci. Publ. Math. No. 57 (1983), 73-212. MR0699058

[RS83] Algebraic Geometry, Proceedings, Tokyo/Kyoto 1982, Eds. Raynaud, M, Shioda, T, Lecture Notes in Math. 1016, Springer-Verlag, Berlin, 1983.

[R85] Hauteurs et isogénies, Séminaire sur les pinceaux arithmétiques (la conjecture de Mordell), L. Szpiro, Astérisque 127 (1985), 199-234. MR0801923

[BLR90] Bosch, Siegfried; Lütkebohmert, Werner; Raynaud, Michel, Néron models. Ergebnisse der Mathematik und ihrer Grenzgebiete (3) [Results in Mathematics and Related Areas (3)], 21. Springer-Verlag, Berlin, 1990. MR1045822

[R94a] Revêtements de la droite affine en caractéristique $p>0$ et conjecture d'Abhyankar. Invent. Math. 116 (1994), no. 1-3, 425-462. MR1253200

[R94b] 1-motifs et monodromie géométrique. Périodes 
p-adiques. Séminaire de Bures, 1988. Astérisque 223 (1994), 295-319. MR1293976

[LLR04] Liu, Q, Lorenzini, D, Raynaud, M. Néron models, Lie algebras, and reduction of curves of genus one. Invent. Math. 157 (2004), no. 3, 455-518. MR2092767

[LLR05] Liu, Q, Lorenzini, D, Raynaud, M, On the Brauer group of a surface. Invent. Math. 159 (2005), no. 3, 673676. MR2125738

[DM69] Deligne, P, Mumford, D, The irreducibility of the space of curves of given genus. Inst. Hautes Études Sci. Publ. Math. No. 36 (1969), 75-109. MR0262240

[E84-86] Ekedahl, T, On the multiplicative properties of the de Rham-Witt complex. I. Ark. Mat. 22 (1984), no. 2, 185-239. MR0765411 On the multiplicative properties of the de Rham-Witt complex. II. Ark. Mat. 23 (1985), no. 1, 53-102. MR0800174 Diagonal complexes and F-gauge structures. Travaux en Cours. [Works in Progress] Hermann, Paris, 1986. MR0860039

[F84] Faltings, G, Endlichkeitssätze für abelsche Varietäten über Zahlkörpern. (German) [Finiteness theorems for abelian varieties over number fields] Invent. Math. 73 (1983), no. 3, 349-366. MR0718935 Erratum: "Finiteness theorems for abelian varieties over number fields". Invent. Math. 75 (1984), no. 2, 381. MR0732554

[H94] Harbater, D, Abhyankar's conjecture on Galois groups over curves. Invent. Math. 117 (1994), no. 1, 1-25. MR1269423

[R90] Ribet, K, On modular representations of $\mathrm{Gal}(\overline{\mathbf{Q}} / \mathbf{Q})$ arising from modular forms. Invent. Math. 100 (1990), no. 2, 431-476. MR1047143

[M77] Mazur, B, Modular curves and the Eisenstein ideal. Inst. Hautes Études Sci. Publ. Math. No. 47 (1978), 33-186. MR0488287

[T04] Tamagawa, A, Finiteness of isomorphism classes of curves in positive characteristic with prescribed fundamental groups. J. Algebraic Geom. 13 (2004), no. 4, 675724. MR2073193

[EGA] Eléments de géométrie algébrique, par A. Grothendieck (rédigés avec la collaboration de J. Dieudonné), Inst. Hautes Études Sci. Publ. Math. No. 4, 8, 11, 17, 20, 24, 28, 32; Grundlehren der mathematischen Wissenschaften, No. 166, Springer Verlag, Berlin, 1971.

[SGA 1, 2, 3, 4, 5, 6, 7] Séminaire de Géométrie Algébrique du Bois-Marie, dirigé par A. Grothendieck, Lecture Notes in Math. 151, 152, 153, 224, 229, 270, 288, 305, 340, 589, Springer-Verlag, Berlin, 1971-1977; Advanced Studies in Pure Math. 2, North-Holland, Amsterdam, 1968; Documents Math. 3; 4; 7, 8, Soc. Math. France, Paris, 2003; 2005; 2011

\section{Bourbaki Talks}

Caractéristique d'Euler-Poincaré d'un faisceau et cohomologie des variétiés abéliennes. Séminaire Bourbaki (1964/66), Exp. no. 286, Dix exposés sur la cohomologie des schémas, 12-30, Adv. Stud. Pure Math., 3, North-Holland, Amsterdam, 1968.

Familles de fibrés vectoriels sur une surface de Riemann (d'après C. S. Seshadri, M. S. Narasimhan et D. Mumford). Séminaire Bourbaki (1966/68), Exp. no. 316, 45-60.

Travaux récents de M. Artin. Séminaire Bourbaki (1968/69), Exp. no. 363, 279-295, Lecture Notes in Math. 175, Springer-Verlag, Berlin, 1971.

Compactification du module des courbes. Séminaire Bourbaki (1970/71), Exp. no. 385, 47-61. Lecture Notes in Math. 244, Springer-Verlag, Berlin, 1971.

Construction analytique de courbes en géométrie non archimédienne (d'après D. Mumford), Séminaire Bourbaki (1972/73), Exposé no. 427, 171-185, Lecture Notes in Math. 383, Springer-Verlag, Berlin, 1974.

Faisceaux amples et très amples, Séminaire N. Bourbaki, 1976-1977 , Exp. no. 493, 46-58, Lecture Notes in Math. 677, Springer-Verlag, Berlin, 1978.

\section{Credits}

Photo of Raynaud lecturing is courtesy of University Paris-Sud.

Photo of Raynaud interview is courtesy of Fred HullinCOMPAS Université Paris-Sud.

Author photo is courtesy of Luc Illusie. 


\section{Memories of a Grandmaster:} Michel Raynaud and the Flattening by Blowing Up

\section{Ahmed Abbes}

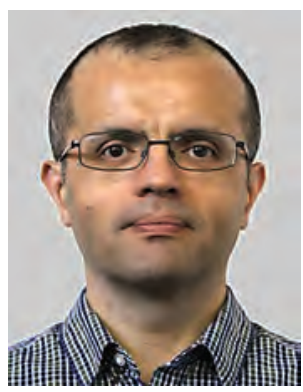

Ahmed Abbes
"A course in algebraic geometry is a course in which one takes out more and more algebra and puts in more and more geometry." This beautiful definition is due to Michel Raynaud. He would often add, with a trace of bitterness and considerable humor: "but these days, students arrive knowing so little algebra there is nothing to take out." His conception of what a course of algebraic geometry should be is perfectly illustrated by his book Anneaux locaux henséliens [5], based on a graduate course given at Orsay in 1969, probably still the best place to learn the notion of étale morphism. Although accessible to beginning students, this book remains, nearly fifty years after its publication, the definitive reference for the notion of a henselian pair, beyond the case of local rings.

The theme of removing more and more algebra and replacing it by more and more geometry is also present in Raynaud's research. A striking example is his joint article with Laurent Gruson, Critères de platitude et de projectivité, Techniques de platification d'un module [8]. When I asked him at some point if he still had any reprints, he answered that there were no more, and that he was very surprised that there had been so much demand. Despite this modesty, so characteristic of Raynaud, this article is one of the masterpieces of algebraic geometry. To this day it remains his most cited article on MathSciNet ${ }^{\circledR}$. In order to describe its main result, I need first to present the notion of flatness to the readers who do not know it. I can do no better than to quote the following excerpt from another of his articles,

Ahmed Abbes is research director at CNRS, Institut des Hautes Études Scientifiques (IHÉS), Bures-sur-Yvette, France. His email address is abbes@ ihes. fr. He thanks Arthur Ogus for help with the English translation.

For permission to reprint this article, please contact: reprint - permission@ams.org.

DOI: http://dx.doi.org/10.1090/noti1755 intended for a wider public, and entitled Grothendieck et la théorie des schémas [7], in which he describes his teacher Grothendieck's encounter of the notion of flatness.

In GAGA [9], Serre gave a wonderful example of a "flat pair," in the course of the passage from algebraic to analytic geometry. With Grothendieck, flatness will play an important role, demonstrated in many ways.

First of all, flatness is at the heart of relative geometry. If one thinks of a morphism $f: X \rightarrow S$ as a family of schemes over fields parameterized by the points of $S$, flatness of $f$ is the most convenient hypothesis assuring that a fiber of $f$ over a point $s$ of $S$ is "precisely" contained in the scheme theoretic closure of a fiber over a generization of $s$. Thus flatness appears as a natural link among the fibers in relative geometry. Nevertheless, flatness is not a completely "geometric" notion; it includes, in the end, an algebraic aspect. For example, if $S$ is local and Artinian, the flatness of $f$ translates simply as the statement that the rings of open affine subsets of $X$ are free modules over the ring of $S$. Furthermore, in the noetherian case, flatness can be tested on Artinian rings, by passing to the limit. This motivated Grothendieck to prove a delicate criterion for flatness, which he suggested to Bourbaki. It appeared in Bourbaki Alg. Com. Chap $3[1] \ldots$.

A technical advantage of the notion of flatness is that, under reasonable finiteness assumptions (noetherianity, finite presentation), a number 
of standard properties of a morphism $f: X \rightarrow S$ can be checked on the fibers, assuming that $f$ is flat. One thus obtains a general method to pass from geometry over a field to relative geometry and conversely, leading to the development of [a] whole chapter-simultaneously useful and easy-of relative commutative algebra....

Flatness, under suitable finiteness conditions, is ideal for the study of local and openness properties. Following the path initiated by Serre in Algèbre locale et multiplicités, [10], Grothendieck systematically "modulates" his statements. Thus a good framework is to consider a morphism $X \rightarrow S$, locally of finite type, and a sheaf of $\mathcal{O}_{X}$-modules of finite presentation $\mathcal{M}$, which is $S$-flat.

As one sees, when Raynaud speaks of a notion in algebraic geometry, it is as if he endows it with a living soul.

One might think that the "good framework" for studying local and openness properties which he describes at the end of the excerpt above is so good that it is exceptional. But in fact in his article with Gruson, he proves that one can always transform a (rather general) relative situation into this good case, by a simple transformation, "blowing up." In order to describe this result in simple terms, I must first do the opposite of Raynaud's approach to teaching algebraic geometry, namely I must return to algebra. Thus let $A$ be a ring, $B$ an $A$-algebra of finite presentation, and $M$ a $B$-module of finite presentation. Raynaud and Gruson are interested in the flatness of $M$ as an A-module. They begin by establishing a criterion that reduces the study of flat $A$-modules to the case of modules that are free and of finite type over smooth $A$-algebras. The precise statement is a little bit technical, but let us try: the module $M$ is $A$-flat if and only if, locally for the étale topology on $\operatorname{Spec}(A)$ and $\operatorname{Spec}(B)$, the $A$-module $M$ admits a finite composition series the successive quotients of which are the A-modules underlying free finite type modules over $A$-algebras which are smooth and with geometrically integral fibers. They then show that one can render $M$ flat over $A$ by performing a blow-up on $\operatorname{Spec}(A)$ and replacing $M$ by its strict transform along this blow-up. Thanks to their criterion, the key case is when the $A$-algebra $B$ is smooth with geometrically integral fibers, in which case one can use the Fitting ideals to make $M$ locally free over $B$. Their results, which have the virtue of extending to the case in which $M$ is merely of finite type over $B$, provide an alternative to Grothendieck's approach in EGA IV to the study of flatness in a relative case [3].

The main motivation of the work of Raynaud and Gruson came from Tate's rigid geometry. Their techniques extend to formal geometry and provide a systematic procedure for transforming problems in rigid geometry into

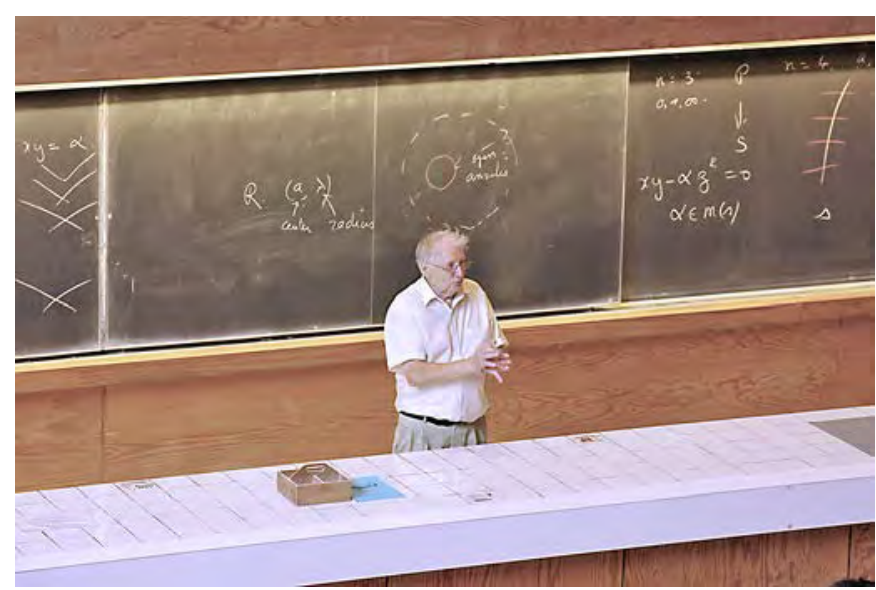

Michel Raynaud during a conference in Rennes, France, July 2009.

problems in algebraic geometry [6]. This approach has proved extremely powerful and has had many applications in arithmetic and in algebraic geometry.

At the beginning of this year, I sent my New Year's greetings to Raynaud and attached to my email an article by my student Quentin Guignard, which gives a new proof of his theorem with Gruson on flattening by blowing-up [2]. I didn't get an answer, and in fact I learned several days later that Raynaud had had an emergency operation because of a brain tumor. I did have the opportunity to meet with him a little later. He had come, with his wife and son, to see the office in the new mathematics building in Orsay that had been intended for him to share with Luc Illusie. With his usual lively humor, he began by teasing Luc about a joint past collaboration [4]. He thanked me for my New Year's greetings and the article, and added in a bantering tone of voice: "but what gave him the idea of finding a new proof, mine is after all well understood." He then summarized it in a few sentences in his fascinating style, so alive and inimitable. He was still amazed by the fact that, to flatten a module, one can reduce to the case in which one can make it locally free. This was, alas, our last meeting.

My strongest memory of him, one that will stay with me forever, goes back to July 2000. We were in Azumino, in the Japanese Alps, for a conference on algebraic geometry. I met him a few minutes before the beginning of his exposé; he was, as was his habit, pacing back and forth, somewhat nervously. I said to him, teasing, "What, at your age?" He looked me in the eye and answered, "This fire, if you lose it, you lose everything."

\section{References}

[1] N Bourbaki. Algèbre commutative, Chapitres 1-9, Hermann (1985).

[2] Q Guignard. A new proof of Raynaud-Gruson's flattening theorem, prepublication (2018), arXiv:1801.01188.

[3] A Grothendieck, J A Dieudonné. Éléments de Géométrie Algébrique, IV Étude locale des schémas et des morphis- 


\section{MEMORIALTRIBUTE}

mes de schémas, Pub. Math. IHÉS 20 (1964), 24 (1965), 28 (1966), 32 (1967).

[4] L Illusie, M Raynaud. Les suites spectrales associées au complexe de de Rham-Witt, Publ. Math. de l'IHÉS, 57 (1983), 73-212. MR0699058

[5] M Raynaud. Anneaux locaux henséliens, Lecture Notes in Mathematics 169, Springer-Verlag (1970). MR0277519

[6] M Raynaud. Géométrie analytique rigide d'après Tate, Kiehl... Mémoires de la Société Mathématique de France, 39-40 (1974), 319-327. MR0470254

[7] M Raynaud. Grothendieck et la théorie des schémas, in Alexandre Grothendieck: a mathematical portrait, Int. Press (2014), 25-34. MR3236918

[8] M Raynaud, L Gruson. Critères de platitude et de projectivité. Première partie: Platitude en géométrie algébrique, Invent. Math. 13 (1971), 1-89. MR0308104

[9] J-P Serre. Géométrie algébrique et géométrie analytique, Ann. Inst. Fourier, Grenoble 6 (1955-1956), 1-42. MR0082175

[10] J-P. Serre. Algébre Locale, Multiplicités: Cours au Collège de France, 1957-1958, Lecture Notes in Mathematics 11, Springer-Verlag (1965). MR0201468

\section{Credits}

Author photo is courtesy of Ahmed Abbes.

Photo of Michel Raynaud by Ahmed Abbes [GFDL (www .gnu.org/copyleft/fd7.htm]) or CC BY SA 4.0 (https://creativecommons.org/licenses/by -sa/4.0)], from Wikimedia Commons.
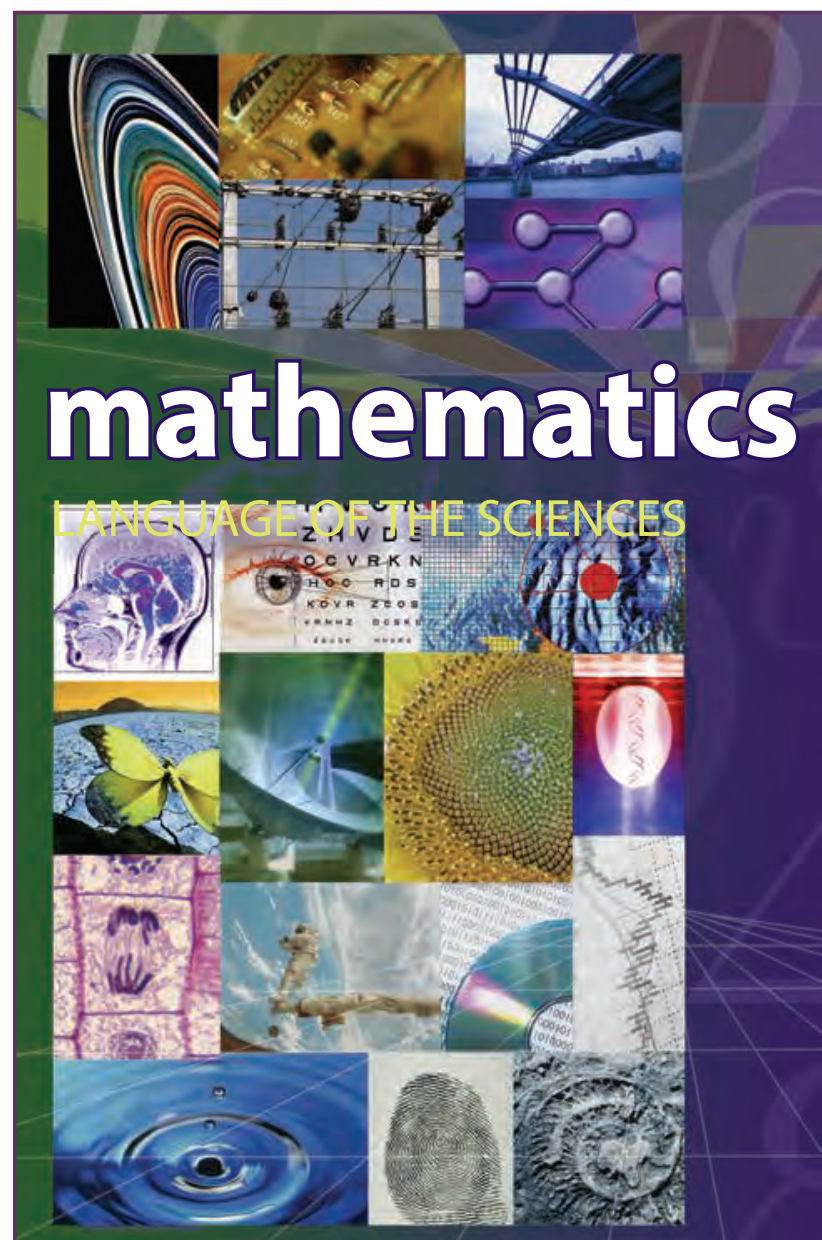

\section{engineering}

astronomy

robotics

genetics

biology

medicine

clinatology

forensics.

statistics

finance

computer science

physics

neuroscience

chemistry

ge010gy

ecology

molecular biology

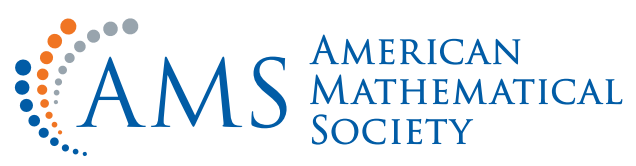




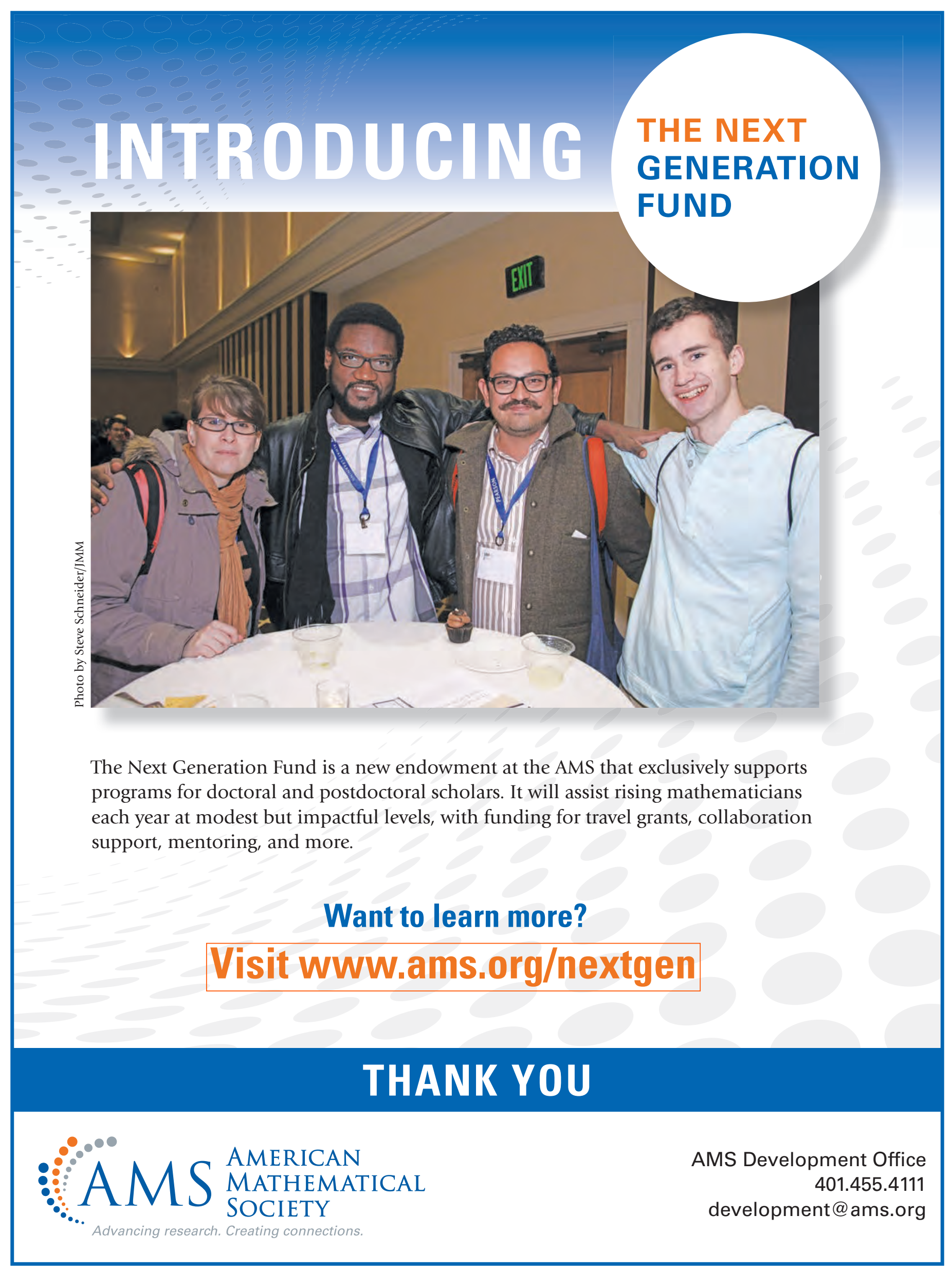


A Survey of Significant Developments in Undergraduate Mathematics Education Over the Past Decade

\section{Ron Buckmire}

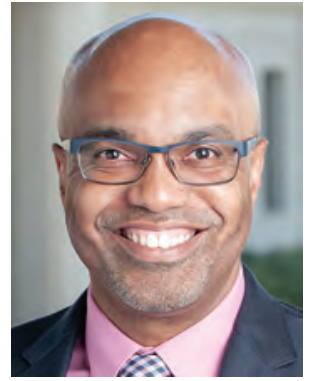

Ron Buckmire

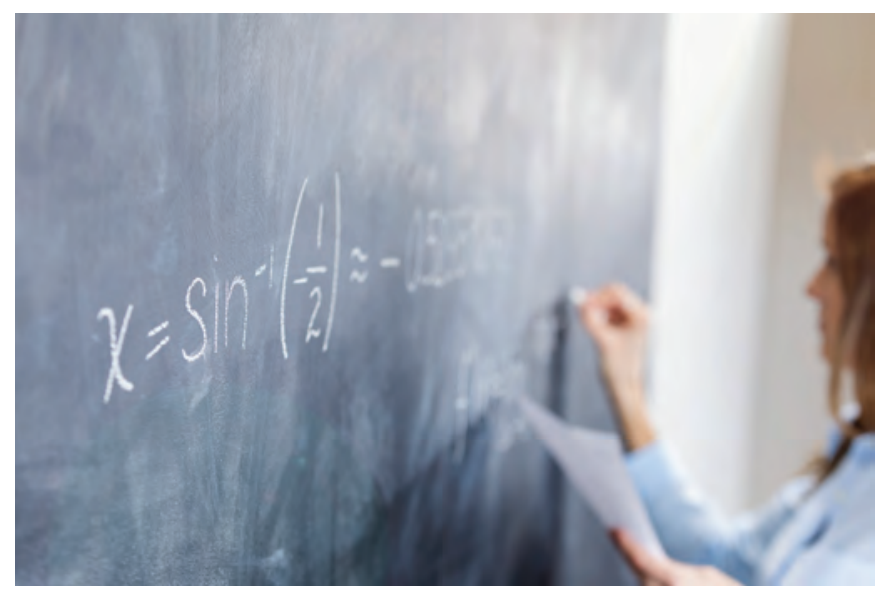

Ron Buckmire is Professor of Mathematics, Associate Dean for Curricular Affairs, and Director of the Core Program at Occidental College in Los Angeles, CA. He was a (permanent) Program Officer in NSF's Division of Undergraduate Education from May 2016 to August 2018 and a (temporary) program officer from August 2011 to August 2013. His email address is ron@oxy. edu.

Communicated by Notices Associate Editor William McCallum.

For permission to reprint this article, please contact: reprint -permission@ams .org.

DOI: http://dx.doi.org/10.1090/noti1761
In this article I hope to provide some thoughtful responses to the organizing question "What are the most significant results, events, or developments in undergraduate mathematics education of the last decade?" To help answer this question, I posed it to approximately two dozen individuals prominent in the mathematics community and received timely responses from about two-thirds. For several years I have been one of the very few individuals in the federal government whose job description included the responsibility to "promote excellence in undergraduate mathematics education for all students in the United States. ${ }^{11}$ In this capacity I have had a specialized perspective on the issues and concerns affecting undergraduate mathematics education and developed a particular network of contacts that I consulted to address the central question of this article. The individuals I surveyed included current and former program officers at the National Science Foundation; staff members (including past and present presidents), committee chairs, and officers of several national mathematics organizations; prominent researchers in undergraduate mathematics education; and other professors of mathematics. I recognize and acknowledge that the responses I received do not comprise a complete or representative sample of the possible answers to this question. There are topics that are over-represented in the subsequent discussion as

\footnotetext{
${ }^{1}$ The mission of NSF's Division of Undergraduate Education is "To promote excellence in undergraduate science, technology, engineering, and mathematics (STEM) education for all students."
} 
well as other topics that are missing or under-represented (e.g. teacher education, upper-division mathematics topics, and major-specific courses like real analysis and abstract algebra).

I have grouped the responses describing the most significant "results, events, or developments" in undergraduate mathematics over the last decade that I received into four distinct categories. My set of labels for these responses are curricular pathways (improving and modifying the trajectory students take to be prepared to be successful in college credit-bearing mathematics courses), demographic changes (addressing the underrepresentation of women and racial/ethnic minorities in all aspects of the mathematics community), pedagogical innovations (recognizing and incorporating active-learning and other evidence-based reforms in the teaching and learning of mathematics), and expanding career options (recognizing that the overwhelming majority of students who major in and take mathematics courses will not go on to become research mathematicians by providing all students with authentic experiences and numerous examples of how mathematics is actually used in non-academic settings).

\section{CURRICULAR PATHWAYS}

\section{David Bressoud, DeWitt Wallace Professor of} Mathematics, Macalester College, Minneapolis, MN; Director, Conference Board of the Mathematical Sciences (2017 to present); President, Mathematical Association of America (2009-2010).

I strongly suspect that the most far-reaching development in undergraduate mathematics of the past decade has gone on under the radar of most readers of the Notices. It is the emergence and widespread adoption of curricular changes typically known as "pathways projects." These arose in recognition of a serious problem: the low degree-completion rates of students who arrive at a two- or four-year undergraduate program with significant mathematical deficiencies. Traditionally, a student has had to pass a course of College Algebra before receiving any kind of degree. Large numbers of students, especially in twoyear colleges but also in our four-year institutions, fail to qualify to start with College Algebra or a comparable course for which they can obtain college credit; instead they first enroll in a pre-college, non-credit-bearing course in mathematics. A study (Bailey et al, 2010) revealed that the prospects for these students have been abysmal, with only $15 \%$ eventually earning credit for College Algebra or another college credit-bearing mathematics course. The numbers of students involved are really quite large. In the fall term of 2015, 1.8 million students were enrolled in a pre-college, non-credit-bearing mathematics course, one million of them in four-year institutions, the others in twoyear colleges. (To get a sense of the scale of this problem there are 550,000 students who took Calculus 1 at the post-secondary institution level and another 800,000 at the high school level in the 2015-16 academic year.) The problem is not just passing the remedial course or courses. Numerous studies have shown that even students who do well in their pre-college non-credit-bearing courses often fail to persist. Even with a $60 \%$ pass rate, which is typical, we often find $40 \%$ or more of those who pass these remedial courses decide not to continue their college education. Students who must pass two or more remedial courses before being allowed into a credit-bearing course are very unlikely to complete a degree. Compounding the problem is the fact that College Algebra, the terminal mathematics course for most of these students, often serves their future academic needs very poorly. The pathways programs, initiated by the Carnegie Foundation for the Advancement of Teaching and supported by the National Science Foundation ${ }^{2}$ and soon followed by the Dana Center at the University of Texas, Austin, have been designed to ensure that all students entering with a need for remediation can enroll in a one-year course that carries college mathematics credit. Instead of College Algebra, the college-level course is usually either statistics or quantitative reasoning. The first semester is designed to prepare students for the credit-bearing course, focusing on the specific competencies, skills, and understandings that students will need for success in the second course. The results are outstanding, with pathways programs now reporting around $70 \%$ of these students successfully completing the sequence.

Jim Ham, President, American Mathematical Association of Two-Year Colleges (AMATYC), 2017-2018. Associate Professor of Mathematics, Delta College, University Center, MI.

The pathways initiative is the most significant trend in undergraduate mathematics education over the last decade. Its goal has been to streamline the developmental mathematics sequence, thereby reducing the time and credit hours necessary for students to attain proficiency in college level mathematics coursework. To achieve this, mathematics departments have created accelerated and corequisite curricular models, changed the initial placement procedures, and altered the developmental mathematics curriculum to align better with each pathway. To date, more than half of the mathematics departments at two-year colleges in the US have implemented pathways-recommended changes.

Jane D. Tanner, President, AMATYC, 2016-2017. Professor Emerita of Mathematics, Onondaga Community College, Syracuse, NY.

In my opinion the most significant development in undergraduate mathematics of the last decade is the realization that every student does not need to take college

${ }^{2}$ DUE-1820830: "Scaling Up through Networked Improvement (SUNI): Testing a practical theory about improving math outcomes for developmental students at scale" and DUE-1322844: "Transforming Students' Mathematical Experiences: Advancing Quality Teaching with Reliability at Scale." 
algebra in order to be successful in their degree programs. The creation of multiple pathways has enabled non-STEM students to avoid the stumbling block of algebra and to complete their degree programs. Our students who are working on STEM degrees now have appropriate content that allows them to be better prepared for the calculus sequence. Increased collaboration among the professional organizations has allowed these pathways to be implemented at all levels of post-secondary mathematics education.

\section{EXPANDING CAREER OPTIONS}

Rachel Levy, Vice President for Education, SIAM, 2015-2018; Professor of Mathematics \& Associate Dean for Faculty Development, Harvey Mudd College, Pomona, CA; Deputy Executive Director, Mathematical Association of America (MAA).

In the past decade there have been significant developments with respect to the ways that undergraduate mathematical sciences education can lead to BIG mathematics jobs (in business, industry, and government). Internships and computer science are both playing a larger role in the undergraduate experience and recruiters are becoming more aware of the value of a degree in the mathematical sciences. Sometimes programming is required for mathematical sciences majors, other times students elect supplementary courses, participate in activities such as hackathons, or learn to program as part of an additional major/minor. Classroom education is being enhanced by workplace and online learning experiences. Students are developing stronger communication skills, and bringing them to bear in mathematical presentations and work environments. Mathematical modeling is growing in $\mathrm{K}-12$. However, the access to each of these opportunities is not yet well-distributed. This presents a challenge for us all.

Padmanabhan Seshaiyer, Associate Dean for Academic Affairs \& Professor of Mathematical Sciences, George Mason University, Fairfax, VA; NSF Program Officer (Division of Mathematical Sciences), 2014-2016.

One of the significant paradigm shifts in undergraduate mathematics education in the last decade has been the willingness of faculty and students to engage in multidisciplinary research moving away from the traditional philosophy of "here is the mathematics, go solve the problem" to a more applied philosophy of "here is the problem, let us find the mathematics to do it." While the intellectual merit has continued to be on developing the fastest, cheapest, or the most accurate method for solving complex systems, there has been an increasing focus on the broader impact of the method to real-world applications. Some examples include probabilistic and stochastic approaches to solve the problem of poaching to the solution of systems of differential equations to study the spread of infectious diseases such as Zika to the use of machine learning in predictive data analytics. This has also helped major organizations to rethink their priorities such as the ten Big Ideas from the National Science Foundation, the fourteen Grand Challenges from the National Academy of Engineering, and the seventeen goals from the United Nations 2030 Sustainable Development Goals. To solve these global challenges there has been a significant focus in undergraduate mathematics education in training the next generation workforce on mathematical modeling, analysis, and simulation. Another major development has been new initiatives and programs to promote the much-needed awareness of celebrating [demographic] diversity in mathematical sciences through social media, movies, and conferences.

Suzanne Weekes, Professor of Mathematical Sciences, Worcester Polytechnic Institute, Worcester, MA; Chair, Committee on Education, SIAM.

I have seen a growing focus on getting students prepared for the non-academic workforce both at the undergraduate and graduate levels. There is certainly a demand in the job market for well-trained problem solvers who can already communicate effectively, who know how to learn new things, and are comfortable working on complex problems especially those involving data and those requiring solid quantitative skills. The way a lot of us are bringing things together for our students is by having our students work on real-world problems coming directly from industry. We have done this with our students at WPI through our senior research projects and in our Research Experiences for Undergraduates (REU) Program in Industrial Mathematics and Statistics for around two decades. Now, in the last five years, mathematics faculty all over the US have been able to participate in the NSF-funded PIC Math program ${ }^{3}$ to help them prepare students at their own universities for industrial careers by engaging their students on research problems that come directly from industry. So far, PIC Math has worked with faculty from over 100 US institutions who have collectively engaged more than 1400 students on industrial mathematics research problems.

William Yslas Vélez, Professor Emeritus, Department of Mathematics, University of Arizona; NSF Program Director (Division of Mathematical Sciences), 19921993.

What do mathematics majors need to be able to compete in today's marketplace? To me it is clear that "one size fits all" is not the answer. Insisting on one program of study for all mathematics majors does not serve the needs of our students. In many departments the traditional course of study in linear and abstract algebra, real analysis, and complex variables needs modification to entice students into the mathematics major and to prepare them to meet the many exciting applications of mathematics. Students

${ }^{3}$ DMS-1722275: "Preparation for Industrial Careers in Mathematical Sciences" and DMS-1345499: "Preparing Mathematical Sciences Students for Business, Industry, and Government Careers (Pre-BIG)." 
majoring in $\mathrm{X}$ find that adding mathematics as another major makes $\mathrm{X}$ more competitive.

\section{PEDAGOGICAL INNOVATIONS}

Benjamin Braun, Associate Professor of Mathematics, University of Kentucky, Lexington, KY; founding editor, AMS On Teaching and Learning Mathematics blog.

Three recent developments stand out in my mind. First, the MAA's NSF-funded National Studies of College Calculus program $^{4}$ has identified characteristics of successful college calculus programs that provide critical information to faculty seeking to improve calculus instruction. Second, the research on inquiry-based learning in college mathematics by Sandra Laursen and her associates [in Ethnography \& Evaluation Research at the University of Colorado Boulder] has solidly established the positive impact that active and inquiry-based learning has on college students. Third, the increasing level of collaboration among professional societies in mathematics over the past decade, most clearly demonstrated in the Common Vision report, has led to a greater degree of coherence in national-level initiatives and support for mathematics education.

Chris Rasmussen, Professor of Mathematics and Statistics, San Diego State University, San Diego, CA; coeditor, International Journal on Research in Undergraduate Mathematics Education.

One major shift in undergraduate mathematics education is the focus on active learning, both from the broader mathematical community and as a specific research focus from the Research in Undergraduate Mathematics (RUME) community. As we found out in the census survey that we did as part of the Progress through Calculus grant, ${ }^{5} 44 \%$ of mathematics departments report that active learning is very important for the precalculus to calculus 2 sequence. I find this remarkable. A decade ago I suspect the percentage of departments reporting this would have been much, much less. Professional societies are stepping up and doing their part, NSF has stepped up and made this a focus, and the RUME community is right there with folks, investigating what this looks like in classrooms, the benefits for learners, the knowledge and dispositions instructors need to do this well, the design of curriculum to support active learning, etc.

Edray Goins, Professor of Mathematics, Pomona College, Claremont, CA; President, National Association of Mathematicians (NAM), 2015 to present.

One of the most significant events in undergraduate mathematics education in the last decade was the adoption of the Active Learning Statement by the CBMS Council in 2016. The Council of the Conference Board of Mathemat-

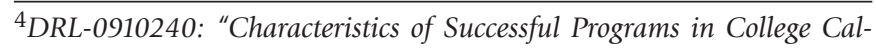
culus."

${ }^{5}$ DUE-1430540: "Progress through Calculus."
}

ical Sciences (CBMS) consists of nearly all the professional organizations in the mathematical sciences, such as the American Mathematical Society (AMS) and the National Association of Mathematicians (NAM). We realized that we needed to make a strong statement about the future of our profession. On July 15, 2016, the CBMS Council unanimously adopted a lengthy position statement on Active Learning. To quote from this 11-page document: "[W]e call on institutions of higher education, mathematics departments and the mathematics faculty, public policy-makers, and funding agencies to invest time and resources to ensure that effective active learning is incorporated into post-secondary mathematics classrooms."

John Haddock, Professor of Mathematics, University of Memphis, Memphis, TN; NSF Program Officer (Division of Undergraduate Education), 2004-2006, 2013-2017.

It is well recognized that Mathematical Sciences departments in the United States often rely heavily on graduate teaching assistants (GTAs) for teaching undergraduate students, and many of these same graduate students enter the workforce with teaching and mentoring undergraduates as an important component of their professional responsibilities. In line with this, there has been an increased interest by departments and professional organizations over the past few years to provide better preparation for GTAs in this realm (e.g. Transforming Post-Secondary Mathematics Education at https://www.tpsemath.org and College Mathematics Instructor Development Source at cominds.maa.org). At the same time, there have been several research efforts regarding how to train these GTAs, leading to the incorporation of education research findings such as understanding more about progressive stages of GTA teaching ${ }^{6}$ and how to moderate them; development, vetting, and utilization of teaching resources ${ }^{7}$; and affirming that GTA training programs certainly can have positive impact in GTAs' students' successes, to cite just a few cases. For a variety of reasons, not the least of which is faculty fear that such programs will have negative effects on graduate student research, many departments have shied away from adopting these new GTA programs. Thus, of particular significance is the research finding that graduate preparation programs for teaching and mentoring undergraduates can be implemented without significantly increasing graduate students' time to graduation as shown in results from another NSF funded project. ${ }^{8}$ In summary, much has been done, but it's just a drop in the bucket of what needs to

\footnotetext{
${ }^{6}$ DUE-1744139: "EAGER: Exploring Mathematics Graduate Teaching Assistants' Developmental Stages for Teaching."

${ }^{7}$ DUE-1432381: "Improving the Preparation of Graduate Students to Teach Undergraduate Mathematics."

${ }^{8}$ DUE-0817537: "Impact of Professional Development Programs on Future STEM Faculty: A Mixed-Methods Longitudinal Study."
} 
be done to improve undergraduate student success in the mathematical sciences.

David Bressoud, DeWitt Wallace Professor of Mathematics, Macalester College, Minneapolis, MN; Director, Conference Board of the Mathematical Sciences (2017 to present); President, Mathematical Association of America (2009-2010).

In 2012, the President's Council of Advisors on Science and Technology (PCAST) castigated the mathematics community for instruction that was "dull and unimaginative," recommending that introductory courses be turned over to "faculty from mathematics-intensive disciplines other than mathematics." Later that year, the National Research Council published its report on the state of Discipline-Based Educational Research. Efforts by the mathematics community were notably absent. It was not the case the mathematics community had been doing nothing to improve instruction, but its efforts were not particularly visible, especially at the research universities that prepare the majority of our science and engineering majors. Research universities are increasingly aware of this need. In 2015 , over $40 \%$ of PhD-granting departments of mathematics acknowledged the use of active learning practices as "very important"; 35\% of PhD-granting departments were at least experimenting with the use of active learning practices in some sections of calculus.

\section{DEMOGRAPHIC CHANGES}

\section{Linda Braddy, Vice President for Academic Affairs,} Tarrant County College; Deputy Executive Director, Mathematical Association of America, 2012-2016.

The increasing awareness of issues related to diversity and inclusion. More and more research is being done on the ways in which our traditional methods of teaching and interacting with students hinder their success in mathematics, particularly students from underrepresented populations. We are far from where we need to be with regards to the use of inclusive pedagogies in our classrooms. Progress is predicated on our willingness to acknowledge and ability to overcome our implicit biases and mistaken notions about student ability and to instead base our perceptions and actions on evidence from current research in the field.

Chris Rasmussen, Professor of Mathematics and Statistics, San Diego State University, San Diego, CA; co-editor, International Journal on Research in Undergraduate Mathematics Education.

Another more recent shift in undergraduate mathematics education is a focus on equity, diversity, and inclusive practices. A few years ago, you would not have found many talks on this subject at the annual Research in Undergraduate Mathematics (RUME) conference, but nowadays these are prominent and very well attended. Consistent with the first theme that is going beyond individual accounts (which often take on a deficit stance), research is examining the gendered and racialized practices across levels (classrooms, departments, institutions, society). I feel very optimistic that this new emphasis in RUME will continue and do some good. The most recent Inquiry-Based Learning (IBL) conference in summer 2018, which was previously associated with the legacy of R. L. Moore, was abound with excellent talks on equity. This is a good sign.

I end this article with a few caveats and observations. First, the responses included here reflect the views of those who submitted them and do not reflect the views of my employers (National Science Foundation or Occidental College). Second, these responses are obviously not from a randomly selected, representative sample of the mathematics community, so there really is no question of whether they span the basis of the entirety of mathematics opinion. Third, the inclusion or exclusion of particular projects or programs does not constitute an endorsement or disparagement by the author. Overall, I believe that the responses included above, taken as a whole, provide some insight into the most important developments in undergraduate mathematics education over the past decade. I hope that readers will use this information to promote excellence in undergraduate mathematics for all students in the next decade and beyond.

References

1. Conference Board of the Mathematical Sciences. (2016). "Activelearningin post-secondary mathematicseducation." Retrieved from https://www.cbmsweb.org/2016 /07/active-1earning-in-post-secondary -mathematics-education.

2. Richelle Blair, Ellen E Kirkman, James W Maxwell. (2018) Statistical abstract undergraduate programs in the mathematical sciences in the United States: 2015 CBMS Survey. American Mathematical Society: Providence, RI.

3. Bressoud, David (2018), "Explosive growth in undergraduate statistics." Retrieved from 1aunchings.blogspot . com/2018/06/explosive-growth-of-advanced . htm 1 .

4. Karen Saxe, Linda Braddy (2016), A Common Vision for Undergraduate Mathematical Science Programs in 2025. Mathematical Association of America: Washington, DC Retrieved from https://www.maa.org/sites /default/files/pdf/CommonVisionfinal.pdf.

5. President's Council of Advisors on Science and Technology (2012), Engage to excel: Producing one million additional college graduates with degrees in science, technology, engineering, and mathematics. Washington, DC: The White House. Retrieved from https://obamawhitehouse.archives.gov/sites/default/files /microsites/ostp/pcast-engage-to-excel -final_2-25-12.pdf.

6. Martha Abell, Linda Braddy, Doug Ensley, Lew Ludwig, Hortensia Soto-Johnson, MAA Instructional Practices Guide. (2018). Mathematical Association of America: Washington, DC Retrieved from https://www.dropbox .com/s/420iptp46iog2w2/MAA_IP_Guide_V1-2 


\section{EDUCATION}

. $\mathrm{ddf} ? \mathrm{~d} 1=0$.

7. GAIMME: Guidelines for Assessment and Instruction in Mathematical Modeling Education, Sol Garfunkel and Michelle Montgomery, editors (2016), Consortium of Mathematics and its Applications (COMAP) and Society for Industrial and Applied Mathematics (SIAM): Philadelphia, PA. Retrieved from https://siam.org/Publications /Reports/Detai1/Guidelines-for-Assessment -and-Instruction-in-Mathematical-Modeling -Education.

8. Sandra L Laursen, Marja-Liisa Hassi, Marina Kogan, Timothy J Weston (2014), "Benefits for women and men of inquiry-based learning in college mathematics: A multi-institution study." Journal for Research in Mathematics Education, 45(4), 406-418.

9. Discipline-Based Education Research: Understanding and Improving Learning in Undergraduate Science and Engineering. Susan R Singer, Natalie R Nielsen, Heidi A Schweingruber, Editors (2012), National Academies Press: Washington, DC.

10. Mark R Connolly, Julia N Savoy, You-Geon Lee, Lucas B Hill (2016), Building a Better Future STEM Faculty: How Teaching Development Programs Can Improve Undergraduate Education. Wisconsin Center for Education Research: Madison, WI. Retrieved from http://1sfss.wceruw.org /finalreport/index.htm7.

11. Thomas Bailey, Dong Wook Jeong, Sung-Woo Cho (2010), "Referral, Enrollment, and Completion in Developmental Education Sequences in Community Colleges." Community College Research Center. Retrieved from https://ccrc.tc.columbia.edu/publications Vreferral-enrol1ment-completion -developmental-education.html.

\section{Credits}

Author photo is courtesy of Ron Buckmire.

FEATURED TITLE FROM 圆圆 HINDUSTAN
BOOK AGENCY

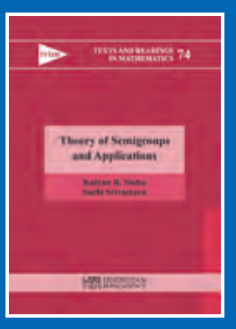

Theory of Semigroups and Applications

Kalyan B. Sinha, Jawaharlal Nehru Centre for Advanced Scientific

Research, Bangladore, India, and Sachi Srivastava, University of Delhi South Campus, New Delhi, India

This book combines the spirit of a textbook with that of a monograph on the topic of semigroups and their applications. It is expected to have potential readers across a broad spectrum that includes operator theory, partial differential equations, harmonic analysis, probability and statistics, and classical and quantum mechanics.

A reasonable amount of familiarity with real analysis, including the Lebesgue-integration theory, basic functional analysis, and bounded linear operators is assumed. However, any discourse on a theory of semigroups needs an introduction to unbounded linear operators, some elements of which have been included in the Appendix, along with the basic ideas of the Fourier transform and of Sobolev spaces. Chapters 4-6 contain advanced material not often found in textbooks but which have many interesting applications, such as the Feynman-Kac formula, the central limit theorem, and the construction of Markov semigroups. The exercises are given in the text as the topics are developed so that interested readers can solve these while learning that topic.

Hindustan Book Agency; 2017; 180 pages; Hardcover; ISBN: 978-93-86279-63-7; List US $\$ 38$; AMS members US $\$ 30.40$; Order code HIN/73

Titles published by the Hindustan Book Agency (New Delhi, India) include studies in advanced mathematics, monographs, lecture notes, and/or conference proceedings on current topics of interest.

\section{Discover more books at bookstore.ams.org/hin.}

Publications of Hindustan Book Agency are distributed within the Americas by the American Mathematical Society. Maximum discount of $20 \%$ for commercial channels.

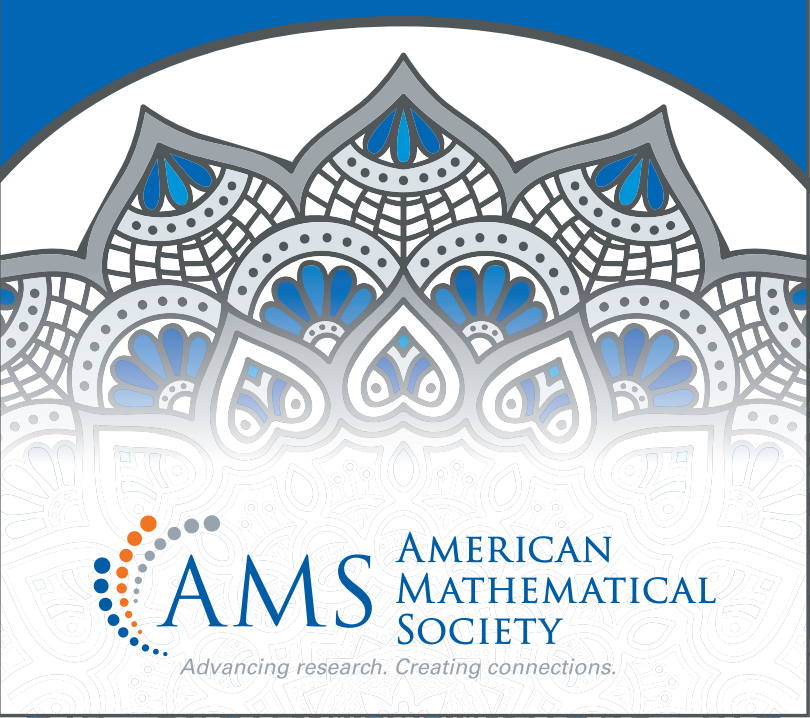




\section{AMERICAN MATHEMATICAL SOCIETY CURRENT EVENTS BULLETIN}

Friday, January 18, 2019, 1:00 PM to 4:45 PM

Room 307, Baltimore Convention Center

Joint Mathematics Meetings, Baltimore, MD

\section{1:00 PM | Bhargav Bhatt University of Michigan}

Perfectoid Geometry and its Applications

Hear about a great new tool for number theory that allows you to treat a prime number like a variable.

\section{2:00 PM \\ Thomas Vidick \\ California Institute of Technology}

Verifying Quantum Computations at Scale:

a Cryptographic Leash on Quantum Devices

Quantum computers would be amazing-but could you trust one?

\section{3:00 PM \\ Stephanie van Willigenburg \\ University of British Columbia}

\section{The Shuffle Conjecture}

Dyck paths, symmetric functions-what's it all about?

\section{4:00 PM \\ Robert Lazarsfeld \\ Stony Brook University}

Tangent Developable Surfaces and the

Equations Defining Algebraic Curves

Some lovely old geometry and an algebraic idea inspired by topology have simplified the proof of a central conjecture about generic Riemann surfaces.

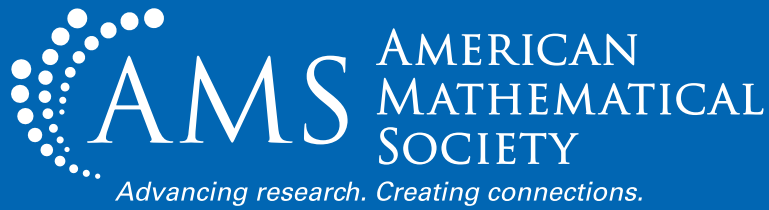

Organized by

David Eisenbud, University of California,
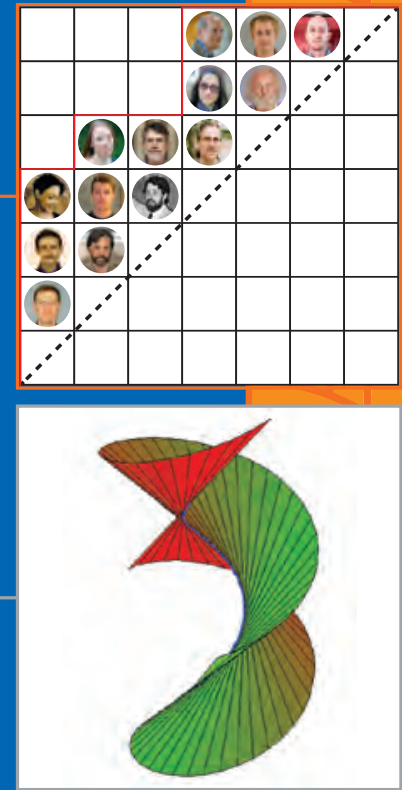


\section{OPINION}

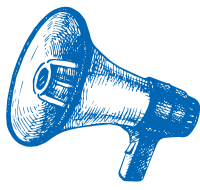

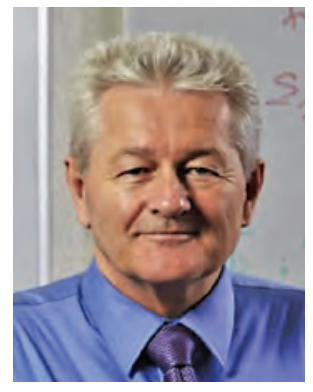

Reinhard Laubenbacher

\section{Mathematics}

Needs AAAS

\section{Reinhard Laubenbacher}

Note: The opinions expressed here are not necessarily those of Notices.

All of us in the mathematical sciences community are aware that the deep connection of mathematics to the sciences has always been a major positive influence in its development. This has never been more true than today, as we are entering another "golden age" of mathematics, driven by the ubiquity of data and the need for new mathematical discoveries that will propel forward both pure and applied mathematics. And most of us agree likewise that it has never been more important than today to communicate the power and beauty of mathematics to non-mathematicians, whether scientists or the general public. Many members of the mathematical sciences community are already engaged with practitioners from other fields or in outreach to the general public, including the American Mathematical Society (AMS) ${ }^{1}$.

The American Association for the Advancement of Science (AAAS) is an organization one of whose broad goals is to "enhance communication among scientists, engineers, and the public." It provides a tremendously useful and greatly underutilized resource for the mathematical sciences community in pursuing this very same goal. The

Reinhard Laubenbacher is the director of the Center for Quantitative Medicine in the UConn School of Medicine; Professor of Computational Biology at the Jackson Laboratory for Genomic Medicine; and AAAS MathSection Secretary. His email address is 1 aubenbacher@uchc. edu.

${ }^{1}$ The AMS Fellowship Program https: //www . ams . org/programs /ams-fe11owships/ams-fellowships.

For permission to reprint this article, please contact: reprint -permission@ams.org.

DOI: http://dx.doi.org/10.1090/noti1751 annual AAAS meeting ${ }^{2}$ provides a ready-made forum for mathematicians to present their work to researchers, and to learn about new developments in other fields. For instance, one participant of this year's annual meeting in Austin, TX, who is head of a science department, told me that she is using scientific symposia at the AAAS annual meeting to identify good speakers from other fields to invite for lectures, as her department is developing a strategic plan to create an interdisciplinary research and graduate program.

The accompanying Opinion piece by H. G. Kaper and H. Engler, reprinted from SIAM News, provides a more detailed view of the opportunties at the annual meeting. In this article, I will provide an overview of other opportunities AAAS presents for mathematicians.

AAAS is an international organization with approximately 130,000 members, focused on the advancement of science, as the name says, through various activities, including interdisciplinary scientific meetings, education and training in outreach and communication, advocacy with government offices, and resources, such as a collection of scientists who will provide timely expertise to journalists who are writing about science. Many K-12 students attend special science programs at the annual meeting every year. Outreach and advocacy for science has never been more important than now, as science and its value to society are increasingly questioned.

AAAS provides resources that can be immensely helpful in accomplishing goals that the professional mathematics societies are also pursuing. For example, besides providing opportunities for interdisciplinary interactions and col-

\footnotetext{
${ }^{2}$ The annual AAAS meeting meetings . aaas .org/program.
} 
laborations, AAAS has a well-recognized and prestigious fellows program that can draw attention to mathematicians' accomplishments $^{3}$. In order to make the most of AAAS as a resource for mathematicians, we need to engage and "provide content."

A large part of success is "just showing up," whether as a member, a conference participant or speaker, or as a member of the governance body of Section A, the mathematics section. Each of the twenty-four AAAS sections receives funds based on membership numbers, and is allotted a certain number of annual fellows the same way. Currently, the mathematics section of the AAAS has slightly more than 2,000 members. It is governed by an eight-member steering group, with Dr. Deborah Lockhart, NSF, as the 2018 section chair. The two most important activities of the steering group are to nominate fellows and to solicit proposals for scientific symposia at the annual AAAS meeting that focus on mathematics in a substantial way. Through generous support from the AMS, as well as AAAS funds, the section can support some or all of the symposium speakers' expenses. Successful symposia are aimed at attracting a broad cross-section of the membership. While this typically favors applied topics, a well-done program on a topic in foundational mathematics could also be very successful. I hear repeatedly from scientists in other fields that they are very curious about research in foundational, "pure" mathematics.

The 2019 AAAS meeting will take place in Washington, DC, February 14-17, and promises to once again give a view of cutting-edge advances across all fields and a vigorous discussion of the place of science in society (see $\mathrm{p} x \mathrm{xx}$ of this issue). We are planning several activities at the meeting, such as presentations by the newly elected AAAS Fellows, a meeting with the editorial staff of Science Magazine, and participation from local and regional undergraduate and graduate mathematics students. There is no better time than now to join AAAS and participate.

\section{Credits}

Author photo by Lanny Nagler for UConn Health.

\section{How to Become Involved}

- Become an AAAS member -you cannot become a fellow without being a member.

- Attend the annual meeting and attend the Section A business meeting to contribute your ideas of how to prominently feature mathematics through AAAS venues.

- Propose a symposium for the next annual meeting (typically three speakers, thirty minutes each).

- Become involved in Section A and AAAS governance activities.

- Use AAAS resources in building your interdisciplinary research or outreach program.

- Attend the AMS-AAAS Liaison Committee meeting at the Joint Mathematics Meetings.

4https://www.aaas.org/membercentral

${ }^{3}$ AAAS Fellowship Programs https://www. aaas.org/genera] -process. 


\section{OPINION}

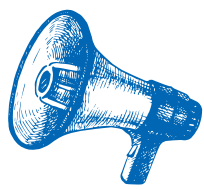

\section{Venturing Outside the Silo}

\section{Hans G. Kaper and Hans Engler}

\section{Note: The opinions expressed here are not necessarily those of Notices.}

Pushing the boundaries of our discipline ensures that mathematics has an impact across fields. As mathematical scientists, we continue to make significant advances and expose new areas of application. But how good are we at communicating the results of our efforts outside our silo? Some scientists are well aware of the contributions we can offer to their discipline; however, too many are not, and consequently invent their own mathematical approaches. Decision-makers rarely recognize mathematics' significant contributions to the "real world." Even well-educated members of the general public believe that most-if not all-mathematical problems were solved a long time ago. Our profession clearly has a communication problem.

We recently organized a scientific session on the "Mathematics of Planet Earth" (MPE) at the 2018 American Association for the Advancement of Science (AAAS) Annual Meeting, held in Austin, Texas, in February 2018. Entitled "MPE: Superbugs, Storm Surges, and Ecosystem Change," our session featured the following three applications of mathematics: "Unseen Enemies: Surveilling, Predicting, and Controlling Epidemic Outbreaks" (Glenn Webb, Vanderbilt University), "Spatial Self-Organization and Its Implications for Ecosystems" (Corina Tarnita, Princeton

Hans Kaper is an affiliate professor of mathematics at Georgetown University. His email address is hgk5@georgetown. edu. Hans Engler is a professor of mathematics at Georgetown University. His email address is eng1er@georgetown.edu.

For permission to reprint this article, please contact: reprint -permission@ams.org.

DOI: http://dx.doi.org/10.1090/noti1752
University), and "Resilient and Sustainable Coasts: How Mathematics and Simulations Play a Role" (Clint Dawson, University of Texas). The meeting allowed us to experience first-hand how other scientists view mathematics and how we can communicate science.

The AAAS is the world's largest general scientific society. It fulfills its dual mission "to advance science and serve society" through many initiatives in science policy, education, and science communication. Thousands of scientists attend AAAS annual meetings, which feature prominent non-scientist speakers, such as the keynote presentation by former vice president Joe Biden at the 2018 meeting. Many science journalists and writers from around the world frequent the meetings, which leads to wide media coverage.

"Mathematics" is Section A among a total of 24 AAAS Sections-recognition of its integral and enabling role in the larger scientific enterprise. Despite this status, very few mathematicians regularly attend the meetings, and mathematics rarely features prominently in the program lineup.

We left Austin with the impression that mathematicians had missed another opportunity to engage with other scientists and the general public. We also witnessed other scientists' increased proficiency in communicating the importance and relevance of their work. Not engaging sufficiently with our peers and failing to regularly and skillfully share our work with a broader audience can only hurt our profession in the long run.

Why does our community not engage more actively with the world outside our silo? Some of the reasons are apparent. We often find it difficult to determine the ques- 
tions that drive projects in other disciplines, or see little opportunity for interesting mathematics in these projects, so we tend to stay within the comfort zone of our own discipline and its reward system. Our training compels us to emphasize correctness and generality over accessibility and illustrative examples, even more so than other sciences. We are used to the inevitable trade-off between accuracy and mathematical tractability of our models, but the price is that our results are harder to communicate. Additionally, mathematicians tend to use their own jargon. Many of us believe that our work is too complicated to explain in terms that are accessible to a general audience. Too often we communicate to address a few dozen peers or perhaps a special community of other scientists, when in fact we could be reaching thousands. Unfortunately, many of us have experienced that those more extensive audiences are not surprised by the narrowness of our message.

\section{Engaging AAAS Offerings}

- In a session titled "Universal and Industrial Quantum Computing," speakers from Google, IBM Research, and TU Delft presented different strategies to increase quantum computation's error resistance and broad availability, and develop new hardware approaches for this task.

- In a flash talk session titled "Developing Robotics to Assist Humans," speakers employed videos and animations to generate a lively discussion about the technical and societal challenges that result from robotic advancement.

- In a plenary address titled "When Facts are Not Enough," Katharine Hayhoe, an atmospheric scientist at Texas Tech University, offered suggestions for connecting with climate skeptics, including bonding over a shared love of gardening or concern for national security.

Effective communication should not be left to professional societies, and we as mathematicians can change our habits. The 2018 AAAS Annual Meeting featured many engaging presentations on a variety of topics, including "What Citizens Think About Science: Survey Data and Implications for Science Communicators," "Science for All: Using Social Media to Take Your Research Around the World," and "Advancing Artificial Intelligence: From the Lab to the Street." See the accompanying inset for additional examples.

Persuasive science communication is a major challenge, especially for our discipline, and requires a shift in our collective thinking. We must recognize that it is not enough to be heard by a gathering of peers; there are broader audiences worth reaching. And we should find ways to prepare our students for this challenge, perhaps by encouraging them to obtain scientific training beyond their narrow specialty or by rewarding them for improved communication skills.

To get you started, consider the following questions. Can you summarize the impact of your work in 200 words, or even a few tweets? Could you explain your research in a 30-second elevator pitch to a dean or provost? Would you be able to get a three-minute video about your work produced and published? By venturing outside our silo, we learn how to strengthen our discipline's communication efforts and spread awareness of mathematics' relevance and value.

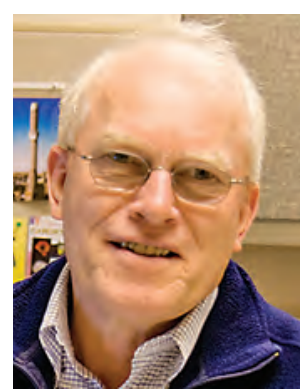

Hans G. Kaper

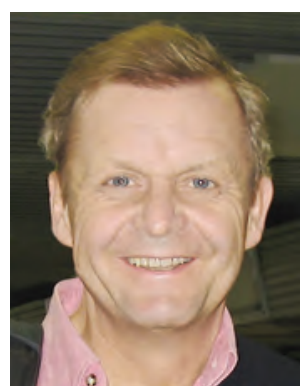

Hans Engler
ACKNOWLEDGMENTS. The authors thank Section A (Mathematics) of the AAAS and the AMS for supporting their participation in the 2018 AAAS Annual Meeting. An earlier version of this article appeared in SIAM News (May 2018, see: https://sinews.siam. org/Details-Page/venturing-outside-the-silo). The authors thank two anonymous reviewers for their insightful comments.

\section{Credits}

Author photos courtesy of the authors. 


\section{AMS Purchase of MAA Press: The View From Acquisitions}

\section{Sergei Gelfand and Stephen Kennedy}

On September 29, 2017, the AMS and the MAA announced an agreement to merge the book programs of the two societies. AMS purchased the book program from the MAA, retaining an ongoing collaborative partnership for the development of content for the book program. In many ways this marriage is a very natural arrangement; AMS Books and MAA Press were, before the partnership, two of the most prominent publishers of English-language mathematics books by and for mathematics professionals. Traditionally, of course, the two presses existed to serve the separate needs of research mathematicians and teaching mathematicians. If that divide ever really made sense, it has grown less significant over recent decades; AMS members have always been, and are increasingly, vitally involved in the teaching and learning of undergraduate mathematics, and MAA members have always had vigorous research careers and have in recent decades increasingly involved their undergraduate students in research. Our missions form a coherent whole and our joint books program reflects that-explain mathematics from the point of view of the professionals who know and love it.

Sergei Gelfand is the Publisher of the AMS and leads the Society's acquisitions efforts. His email address is sxg@ams. org.

Stephen Kennedy was the Senior Acquisitions Editor for MAA Press and now works for AMS as acquisitions specialist for MAA Press titles. His email address is kennedy.maapress@ams.org.

For permission to reprint this article, please contact: reprint -permission@ams.org.

DOI: http://dx.doi.org/10.1090/noti1781
Both societies have, in recent years, been engaged in deliberate efforts to expand and diversify the types of books each produced. The MAA, leveraging its expertise in extraordinary exposition and pedagogy, has made a concerted effort to produce a full catalogue of widely adoptable undergraduate textbooks. The AMS, as a natural outgrowth of its long history of publishing excellent graduate textbooks (and up-to-the-minute monographs), has initiated an undergraduate textbook line and the expository Student Mathematics Library to bolster its catalogue of titles aimed at undergraduates. Simultaneously, AMS has begun an initiative to produce titles for the general public, including a remarkable collection of children's books and several volumes of Math Circle activities. With this growth came an increasing overlap of mission and a realization within the governance structures of both societies that we could be more effective working together by amplifying resonances and reducing redundancies. The marketing capabilities and the world-wide reach and reputation of the AMS give the MAA an extraordinary opportunity to put MAA books in front of an enormously larger market; the AMS gains increased visibility in the North American undergraduate mathematics world and a collection of nearly 300 titles in a market into which the Society hopes to expand.

Both the AMS and the MAA have long relied on volunteer editorial boards to ensure the quality of our books. That will not change with this new arrangement. AMS and MAA book series will continue to have editorial boards appointed by their respective governance structure. And those boards continue to be involved in evaluating and editing their respective manuscripts. The AMS book program at approximately 75 titles per annum pre-merger 
was significantly larger than the MAA's (12-15 books per year). Given the AMS's larger infrastructure, all business functions are now handled by AMS. The MAA Press imprint will be retained, and all MAA titles will bear both societies' imprints. The AMS will preserve the identities of the two presses, while seeking ways to grow both programs symbiotically. AMS employs an acquisitions specialist to take primary responsibility for MAA Press titles.

The publication of undergraduate textbooks is one of the most significant services to mathematics the AMS-MAA book program can provide. Both societies (and within AMS, the acquisitions team in particular) take that responsibility to the community extremely seriously. The commercial textbook market too often lets the professoriate down: with distressing frequency, prices are too high, quality control is slipshod, and editions are renewed with bewildering speed. Market forces seem to dictate what's available instead of the mathematical and pedagogical needs of students. To ameliorate these problems, each society publishes an undergraduate textbook series. We have each made a rigorous effort to maintain high quality of exposition and production while, simultaneously, trying to keep costs to students reasonable. The newly constituted AMS-MAA acquisitions team is engaged in an ongoing effort to obtain the highest quality textbooks possible for our textbook series. We are also attempting to preserve the distinct identities of the existing series: the AMS Pure and Applied Undergraduate Texts series will focus on upper-division courses for students primarily at research universities preparing for a professional career involving mathematics; the MAA Textbooks series will aim at the four-year college market and will include books for non-standard courses and books featuring innovative pedagogical strategies.

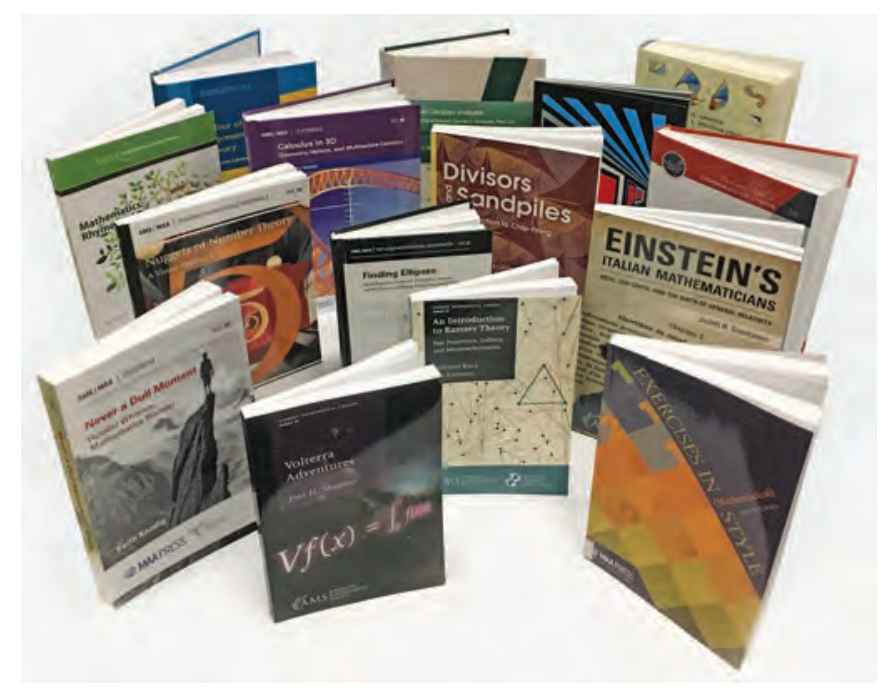

We'd like to take this opportunity to familiarize Notices readers with the MAA Press book catalogue. Besides the Textbooks series, there are six other MAA book series now published by AMS.

The MAA's three expository book series reflect the Association's historical commitment to accessible communication of mathematics. The Anneli Lax New Mathematical Library (NML) produces books primarily targeted at high school students and their teachers. Oleg Ivanov's Portal through Mathematics and The Riemann Hypothesis by Roland van der Veen and Jan van de Craats are typical volumes. The former is a collection of more than 200 puzzles and problems chosen for their capacity to surprise and delight; the latter was written to accompany a summer-enrichment course for advanced high school students. In spite of the intended target, it is often the case that NML volumes can be enjoyed by professionals; it is worth highlighting John McCleary's Exercises in (Mathematical) Style in this context: an homage to Raymond Queneau's Exercises in Style, it tells 99 stories of the binomial coefficients in a variety of voices, styles, and literary forms.

The Dolciani Mathematical Expositions aim to exposit areas of undergraduate mathematics in a way that can be appreciated by both professionals and students. Some typical titles are Larry Lehman's Quadratic Number Theory, Andy Simoson's Exploring Continued Fractions, and Ray Rosentrater's Varieties of Integration. Dolcianis can be used as textbooks; more often they provide enrichment reading for undergraduates or for faculty desiring to expand their understanding.

The Carus Mathematical Monographs is the MAA's Cadillac series featuring exquisite exposition for professionals. The most recent Carus is a beautiful example: Finding Ellipses by Ulrich Daepp, Pamela Gorkin, Andrew Shaffer, and Karl Voss is a surprising mélange of complex analysis, projective geometry, and linear algebra. The typical Carus is written at a level accessible to a reader with some exposure to graduate-level mathematics and is designed to beguile and induce that reader to further study.

The MAA's Classroom Resources Materials (CRM) series provides undergraduate faculty with a wide variety of materials to enrich their pedagogy. Some typical recent titles are Tactile Learning Activities in Mathematics by Julie Barnes and Jessica Libertini and the forthcoming Mathematics for Social Justice by Gizem Karaali and Lily Khadjavi. Both volumes contain a number of classroom-tested modules and activities that, in the former case, feature a physical component and, in the latter case, address an issue of social justice. Included in CRM are a number of possible textbooks featuring unusual content or pedagogy: Discovering Discrete Dynamical Systems by Johnson, Madden, and Sahin is a complete Inquiry-Based Learning course on discrete dynamics, while Introduction to the Mathematics of Computer 


\section{AMS COMMUNICATION}

Graphics by Nathan Carter is designed to accompany a firstyear college course on that topic.

MAA Problem Books are collections of contest problems and solutions usually accompanied by tips on succeeding in competitions. Typical volumes collect Putnam and American Mathematics Competitions problems. (Both competitions are organized by the MAA.) Notable in this series are James Tanton's Clever Study Guides, volumes on secondary topics for teachers and students organized around AMC problems.

Finally, Spectrum is the MAA series of volumes for recreational reading by professionals; it includes memoirs, history, biography, and the occasional piece of fiction. David Zitarelli's forthcoming history of mathematics in North America and Keith Kendig's biography of Hassler Whitney are representative.

The united presses of the MAA and the AMS, with our mutual ethic of service to the profession, intend to jointly continue our long histories of producing high quality mathematical monographs, textbooks, and expositions. As the world's largest mathematics publisher run completely by mathematicians we take very seriously our mission of protecting, preserving, and extending our subject. We aspire to publish the most significant works of mathematics, of mathematics pedagogy, and, more generally, of mathematical culture. The new joint acquisitions team of AMS and MAA consisting of the authors and our colleagues Eriko Hironaka and Ina Mette looks forward with excitement and enthusiasm to taking advantage of the resources and reputations of our two organizations to build the most prestigious and significant catalogue of mathematics books in the world. We invite you to participate in this effort by sending us your manuscripts, by adopting our textbooks, and by enjoying our books.

\section{Credits}

Photo of Sergei Gelfand is by Eric Buck.

Photo of Stephen Kennedy is courtesy of Stephen Kennedy. Photo of books in the AMS, AMS-MAA book programs is by Courtney Rose.

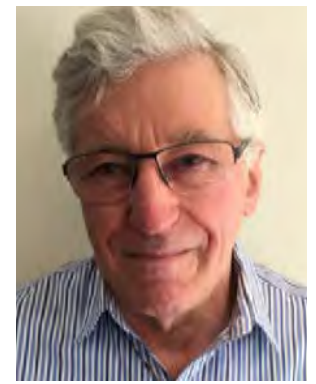

Sergei Gelfand

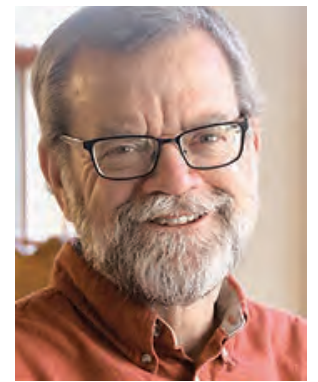

Stephen Kennedy

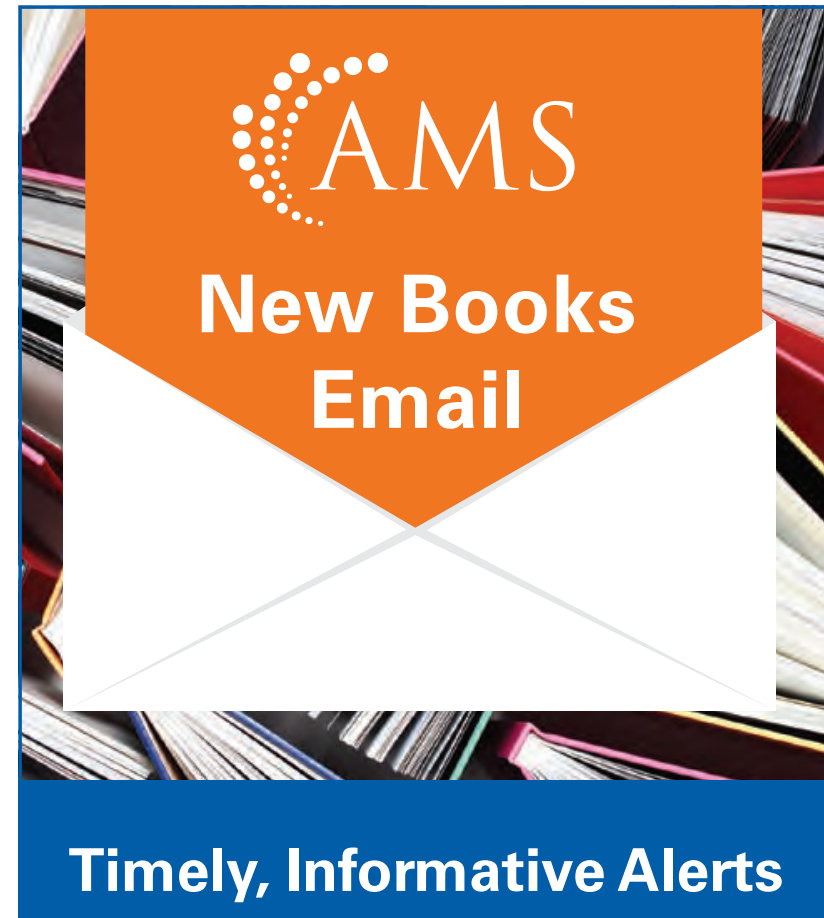

The AMS Bookstore New Books email alert is the best way to keep current with new developments in your field and learn about forthcoming and recently published titles. These monthly mailings allow you to:

- Access each book's abstract page on the AMS Bookstore

- Preview chapter samples, Tables of Contents, Indexes, and author supplemental materials

- Learn about Bookstore sales, special discounts, publishing highlights, and more

It's convenient, it's free, and you can unsubscribe at any time. Sign up today!

WWW.ams.org/ bookstore-email-list 


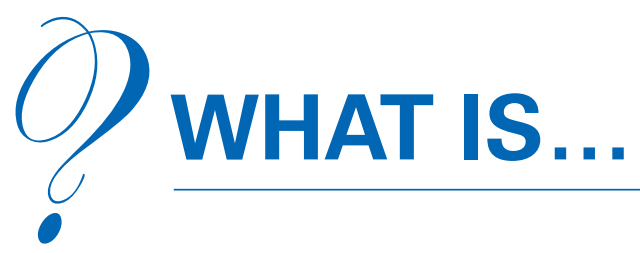

\section{a cryptographic Boolean function?}

\section{Lilya Budaghyan and Pantelimon Stănică}

As one of the most fundamental objects investigated in pure and applied mathematics and computer science, the notion of a Boolean function was introduced about 150 years ago in the context of fundamental mathematics and mathematical logic by an English mathematician George Boole (1815-1864). Boole's treatment of algebra of logic (now known as Boolean algebra) in his The laws of thought [3] laid the foundation for the design of modern digital computer circuits. For positive integers $n$ and $m$, a vectorial, or $(n, m)$ - Boolean function is a map from the finite field $\mathbb{F}_{2^{n}}$ (or the vector space $\mathbb{F}_{2}^{n}$ ) to $\mathbb{F}_{2^{m}}$ (or $\left.\mathbb{F}_{2}^{m}\right)(m=1$ corresponds to a Boolean function). Since the middle of the twentieth century, with the rapid development of information and communication technology, Boolean function theory has become an important tool for solving problems of analysis and synthesis of discrete devices which transform and process information, in particular, in cryptography. In this article, we give an overview of the main concepts and problems in the area of cryptographic Boolean functions from the last 40 years.

To respond to a need for ensuring security of electronic data, in 1973 the US National Bureau of Standards (now,

Lilya Budaghyan is a research director of the project "Optimal Boolean Functions" and a head of Selmer Center-Reliable Communication Group at the University of Bergen, Norway.

Her email address is Li 1ya. Budaghyan@uib. no.

Pante Stănică is professor of Applied Mathematics and Associate Chair for Research at Naval Postgraduate School in Monterey, California, USA. His email address is pstanica@nps. edu.

Communicated by Notices Associate Editor Emilie Purvine and Cesar E. Silva.

For permission to reprint this article, please contact:

reprint-permission@ams.org.
National Institute of Standards and Technology - NIST) issued a call for strong encryption primitives that would become a government-wide standard. Since all submissions were unsuitable that year, NBS re-issued the call and in 1974 a team from IBM submitted a modified version of their cipher Lucifer (designed in 1971). After a year of intense collaboration between IBM and NSA, Data Encryption Standard (DES) was approved, and then published in 1975 in the Federal Register [20]. The call, submission and collaborative effort to approve and publish DES marks the start of intense work on cryptography in academia.

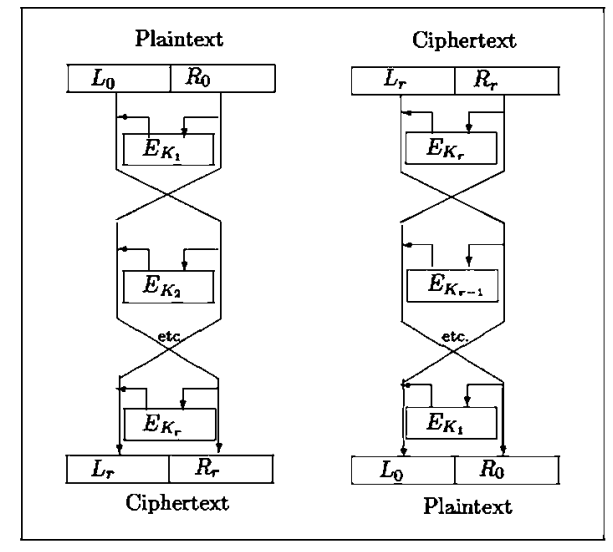

Feistel Cipher with $r$ rounds; Reprinted from [10].

In modern society, exchange and storage of information in an efficient, reliable and secure manner is of fundamental importance. Cryptographic primitives are used to protect information against eavesdropping, unauthorized changes and other misuse. The security of any symmetric cipher relies on components like the substitution $S$-boxes. 
As an example, let the Feistel scheme (one of the two major schemes in addition to Substitution-Permutation Networks) [10] for DES depicted in Fig. 1: we split the input block into two halves $L_{i-1}$ and $R_{i-1}, 1 \leq i \leq r$, and go through a sequence of "rounds" with $L_{i}=R_{i-1}$, $R_{i}=L_{i-1} \oplus E_{K_{i}}\left(R_{i-1}\right)=R_{i-2} \oplus E_{K_{i}}\left(R_{i-1}\right)$, where $\oplus$ is the binary addition and $E_{K}(R)$ is an encryption function using a key $K$ on the block $R$ ).

A substitution or an $S$-box (part of the $E_{K}$ 's above) is nothing else than a cryptographic Boolean function (CBF), that is, a Boolean function possessing some optimal (or near optimal) cryptographic properties. The $S$-boxes introduce confusion (as defined by Shannon in 1949 in his seminal "Communication Theory of Secrecy Systems" [21], where he also considered diffusion - "mixing" the properties of the plaintext into the ciphertext) in the system. Among the most important classes of CBF are the so-called bent, almost perfect nonlinear, almost bent, crooked, correlation immune and resilient functions $[4,7,8,10,16,23]$. Defined for cryptographic purposes starting in the 70's, these turned out to be known for many decades in the context of other domains of mathematics and information theory such as coding theory, sequence design, commutative algebra, combinatorics and finite geometry. During recent years more applications of these beautiful objects have been found, and, we believe, many more are still to be discovered. In spite of the universality of these functions and long history of study not much is known about some of these classes (the case of almost perfect nonlinear and almost bent functions, for instance) and just a few families of such functions have been constructed.

For most cryptographic attacks on block ciphers (like DES, or the current standard, Advanced Encryption Standard - AES) there are certain properties of functions which measure the resistance of the $S$-box to these attacks. One of the most efficient cryptanalysis tools for block ciphers, the differential attack introduced by Biham and Shamir [2], is based on the study of how differences in an input can affect the resulting differences in the output. An $(n, m)$ function $F$ is called differentially $\delta$-uniform if the derivative equation $D_{a} F(x)=F(x+a)-F(x)=b$ has at most $\delta$ solutions for every $a \in \mathbb{F}_{2^{n}}^{*}$ and $b \in \mathbb{F}_{2^{m}}$. Functions with the smallest possible differential uniformity contribute an optimal resistance to the differential attack. In this sense, differentially $2^{n-m}$-uniform functions, called perfect nonlinear (PN), are optimal. However, PN functions exist only for $n$ even and $m \leq n / 2$. For the important case of $n=$ $m$, differentially 2-uniform functions, called almost perfect nonlinear (APN), have the smallest possible differential uniformity. Another powerful attack on block ciphers is the linear cryptanalysis proposed by Matsui [15], which is based on finding affine approximations to the action of a cipher. The nonlinearity of an $(n, m)$-function $F$ is the minimum Hamming distance between all components of
$F$ and all affine Boolean functions in $n$ variables. The nonlinearity quantifies the level of resistance of the function to the linear attack: the higher the nonlinearity, the better the resistance of $F$. Functions whose nonlinearity reaches the universal upper bound are called bent. All bent functions are also PN and vice versa, that is, these functions have optimal resistance against both linear and differential attacks. If $m=n$, PN (or bent) functions do not exist, but if $n$ is also odd, functions with the best possible nonlinearity are called almost bent (AB). When $n$ is even the tight upper bound on nonlinearity is still to be determined. All $A B$ functions are APN, but the converse is not true in general.

There are very few known classes of bent functions in the almost 40 years they have been studied (or more, as difference sets in elementary Abelian 2-groups), and we mention here the constructions of Rothaus, Maiorana, McFarland, Dillon and Carlet (see the excellent survey [9]). The actual count is known for all even dimensions $\leq 8$. For example, for $n=8$, there are approximately $2^{106.2}$ bent functions (in the class of all $2^{2^{8}}=2^{256}$ Boolean functions), but the known bent constructions account only for about $2^{85}$ of those, so there are many more constructions we have not been able to find yet. In spite of not being balanced, there are many properties on bent functions that make them so desirable. For example, the derivative of a bent function is always balanced in any direction, which is quite important for differential cryptanalysis; they have excellent propagation characteristics, since if one changes any nonzero number of bits in the input of a bent function, the output changes with probability $1 / 2$, a desirable property for (fast) correlation attacks. These are just a handful of cryptographic properties connected to bentness, but bent functions are also useful for coding theory. Since they generate difference sets, they can be used to construct a symmetric design. They also give rise to the Kerdock codes, and some other robust error detecting codes. For even dimension, bent functions attain the covering radius of the first-order Reed-Muller code. They have flat absolute values with respect to the Walsh-Hadamard transform (a discrete Fourier transform). One could consider different transforms, and impose flatness of the corresponding values. For example, the choice of the nega-Hadamard transform is motivated by local unitary transforms that play an important role in the structural analysis of pure $n$-qubit stabilizer quantum states, as argued by Riera and Parker [18] in 2006 .

The nonlinearity and the differential uniformity (and, therefore, bentness, PNness, APNness and ABness), are invariant under affine, extended affine and CCZ-equivalences (in increasing order of generality). Two functions $F$ and $F^{\prime}$ are affine equivalent (EA), if $F^{\prime}=B \circ F \circ A(A, B$ are affine permutations) and extended affine equivalent (EAequivalent $)$, if $F^{\prime}=B \circ F \circ A+C$ ( $C$ is just affine). They are 
called CCZ-equivalent if their graphs are affine equivalent. CCZ-equivalence is the most general known equivalence relation of functions which preserves the nonlinearity and differential uniformity. Although CCZ-equivalence is a very powerful method for constructing functions, very little is currently known about CCZ-equivalence classes of the known APN functions (in particular, of all power APN functions except Gold case) whether they are larger than EA-equivalence classes (and EA-equivalence classes of their inverses when a function is a permutation). In general, identifying situations in which CCZ-equivalence reduces to EA-equivalence is useful. It is already known that for all Boolean functions and for all bent functions CCZ-equivalence and EAequivalence coincide, while for quadratic power APN and $\mathrm{AB}$ functions CCZ-equivalence is strictly more general.

The classification of bent, $\mathrm{APN}$ and $\mathrm{AB}$ functions is a hard open problem. Complete classification for APN and $\mathrm{AB}$ functions over $\mathbb{F}_{2^{n}}$ is known only for $n=5$. For bent functions the classification is done nowadays up to $n=8$. The reason the computation is difficult is because the space of all Boolean functions in $n$ variables is doubly exponential, that is, its cardinality is $2^{2^{n}}$. There are only a few infinite classes of $\mathrm{APN}$ and $\mathrm{AB}$ functions known (e.g., four classes of $A B$ power functions, six classes of $A P N$ power functions, eleven classes of quadratic $\mathrm{APN}$ and $\mathrm{AB}$ functions constructed recently; for $6 \leq n \leq 9$ there is a large list of quadratic $\mathrm{APN}$ and $\mathrm{AB}$ functions, but infinite classes are still to be determined). It was conjectured by Dobbertin in 1999 that classification of APN and AB power functions is complete, but the proof is yet to be determined.

Recent advances in APN functions have made a prominent impact on the theory of commutative semifields. In spite of considerable work, only two previously known infinite families of commutative semifields (that are not finite fields) of order $p^{n}, p$ an odd prime, were constructed by Dickson [11] and Albert [1]. A few new such infinite families have been constructed using families of quadratic APN functions (see for instance [6]). Hence, results on the classification of APN and PN functions have important consequences on our understanding of commutative semifields and projective planes.

Quadratic $\mathrm{AB}$ permutations are of interest for combinatorial analysis. A mapping $F$ from $\mathbb{F}_{2^{n}}$ to itself is called crooked if $\left\{D_{a} F(x): x \in \mathbb{F}_{2^{n}}\right\}$ is the complement of a hyperplane, for all $a \in \mathbb{F}_{2^{n}}^{*}$. Every crooked function gives rise to a distance regular rectagraph (a graph without triangles in which every pair of vertices at distance 2 lies in a unique 4-cycle) of diameter 3 , and every quadratic $A B$ permutation (taking 0 value at 0 ) is crooked. The converse is not known, that is, whether a crooked function is necessarily a quadratic $\mathrm{AB}$ permutation. There are not too many constructions of rectagraphs known, especially rectagraphs of small diameter. Hence such functions provide not only interesting building blocks for symmetric cryptosystems but also provide new distance regular rectagraphs. Nowadays only two families of crooked functions are known. One constructed in 1968 by Gold [13] in his investigation of sequence designs and rediscovered in 1993 by Nyberg [17] in the context of cryptography, giving rise to Preparata graphs. The other one is the family of binomials constructed in 2008 by Budaghyan, Carlet and Leander [5].

For $n$ odd all power APN functions are permutations. Certainly, one wonders whether there exist APN permutations over $\mathbb{F}_{2^{n}}$ in the case when $n$ is even, and in [12] Dillon et al. constructed an APN permutation for $n=6$ applying CCZ-equivalence to a quadratic APN function. It is a surprising result since quadratic APN functions themselves cannot be permutations, and it is one of the few results showing that CCZ-equivalence changes properties of functions considerably. Now it is a big challenge to construct APN permutations for larger $n$, or better yet, construct infinite families of such functions.

We end our Boolean functions snapshot with an extension that has gained increasing attention in the last few years, stepping off outside of the binary world. We mention here the generalized Boolean functions, defined on $\mathbb{F}_{2^{n}}\left(\right.$ or $\mathbb{F}_{2}^{n}$ ) with values in integers modulo $q \geq 2$, or $p$-ary Boolean functions, that is, functions defined on $\mathbb{F}_{p^{n}}$ with values in $\mathbb{F}_{p^{m}}$, where $p$ is a prime number. An important topic here is the study of generalized bent functions, which are those whose generalized (using powers of a complex root of 1) Walsh-Hadamard transform absolute values are constant. Multicarrier communications and modulation schemes is a major research topic that has been around for slightly over two decades, rooted in the explosive growth of the Internet, which in turn has increased the demand for wired and wireless high data rates. A major problem with the multicarrier modulation in general and the OFDM (Orthogonal Frequency Division Multiplexing) system in particular is the high peak-to-average power ratio (PAPR) that is inherent in the transmitted signal. Schmidt in 2009 [19] showed that one can achieve a perfect modulation (with respect to the PAPR) if one uses the sequence associated to a generalized bent Boolean function. Researchers' attempts in constructing or describing these generalized bent functions culminated in 2017 with their complete characterization in terms of classical bent Boolean functions in two independent works $[14,22]$.

In this article we gave a brief overview of some of the main concepts, results and the problems we face in cryptographic Boolean functions' research. It is by no means all inclusive, rather it is an attempt to whet the appetite of the mathematician as well as the practitioner to delve more in this area. 


\section{References}

[1] A. A. Albert, On nonassociative division algebras, Trans. Amer. Math. Soc. 72 (1952), 296-309; MR0047027.

[2] E. Biham, A. Shamir, Differential Cryptanalysis of the Data Encryption Standard, Springer-Verlag, 1993.

[3] G. Boole, An Investigation of the Laws of Thought on Which are Founded the Mathematical Theories of Logic and Probabilities, Macmillan, 1854; Reprinted with corrections, Dover Publications, New York, NY, 1958.

[4] L. Budaghyan, Construction and Analysis of Cryptographic Functions, Springer-Verlag, 2014; MR3290040.

[5] L. Budaghyan, C. Carlet, G. Leander, Two classes of quadratic APN binomials inequivalent to power functions, IEEE Trans. Inf. Theory 54:9 (2008), 4218-4229; MR2450779.

[6] L. Budaghyan and T. Helleseth, New perfect nonlinear multinomials over $\mathbb{F}_{p}^{2 k}$ for any odd prime $p$, Proc. Int. Conf. on Sequences and Their Applic. SETA 2008, LNCS 5203, pp. 403-414, 2008; MR2646419.

[7] C. Carlet, Boolean functions for cryptography and error correcting codes, In: Y. Crama, P. Hammer (eds.), Boolean Methods and Models, Cambridge Univ. Press, Cambridge, pp. 257-397, 2010.

[8] C. Carlet, Vectorial Boolean Functions for Cryptography, In: Y. Crama, P. Hammer (eds.), Boolean Methods and Models, Cambridge Univ. Press, Cambridge, pp. 398-472, 2010.

[9] C. Carlet, S. Mesnager, Four decades of research on bent functions, Designs, Codes \& Cryptogr. 78 (2017), 5-50; MR3440222.

[10] T. W. Cusick, P. Stănică, Cryptographic Boolean Functions and Applications (Ed. 2), Academic Press, San Diego, CA, 2017; MR3644644.

[11] L. E. Dickson, On commutative linear algebras in which division is always uniquely possible, Trans. Amer. Math. Soc. 7 (1906), 514-522; MR1500764.

[12] K. A. Browning, J. F. Dillon, M. T. McQuistan, A. J. Wolfe, An APN Permutation in Dimension Six, Post-proc. 9-th Int. Conf. Finite Fields and Their Applic. Fq'09, Contemporary Math., AMS, v. 518, pp. 33-42, 2010; MR2648537.

[13] R. Gold, Maximal recursive sequences with 3-valued recursive crosscorrelation functions, IEEE Trans. Inf. Theory 14 (1968), 154-156.

[14] T. Martinsen, W. Meidl, S. Mesnager, P. Stănică, Decomposing generalized bent and hyperbent functions, IEEE Trans. Inform. Theory 63:12 (2017), 7804-7812; MR3734198.

[15] M. Matsui, Linear cryptanalysis method for DES cipher, Adv. Crypt. - EUROCRYPT'93, 386-397.

[16] S. Mesnager, Bent functions. Fundamentals and Results, Springe-Verlag, 2016; MR3526041.

[17] K. Nyberg, Differentially uniform mappings for cryptography, Adv. Crypt. - EUROCRYPT'93, LNCS 765 (1994), 55-64; MR1290329.

[18] C. Riera and M. G. Parker, Generalized bent criteria for Boolean functions, IEEE Trans. Inf. Theory 52:9 (2006), 4142-4159; MR2298538.

[19] K.-U. Schmidt, Quaternary Constant-Amplitude Codes for Multicode CDMA, IEEE Trans. Inf. Theory 55:4 (2009), 1824-1832; MR2582768.
[20] B. Schneier, Applied Cryptography: Protocols, Algorithms and Source Code in C, John Wiley \& Sons, Inc., 2015; MR3587912.

[21] C. Shannon, Communication Theory of Secrecy Systems, Bell System Technical Journal 28:4, 656-715, 1949.

[22] C. Tang, C. Xiang, Y. Qi, K. Feng, Complete characterization of generalized bent and $2^{k}$-bent Boolean functions, IEEE Trans. Inform. Theory 63:7 (2017), 4668-4674; MR3666982.

[23] N. Tokareva, Bent Functions. Results and Applications to Cryptography, Academic Press, San Diego, CA, 2015; MR3362707.

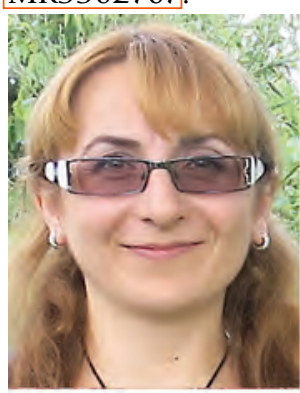

Lilya Budaghyan

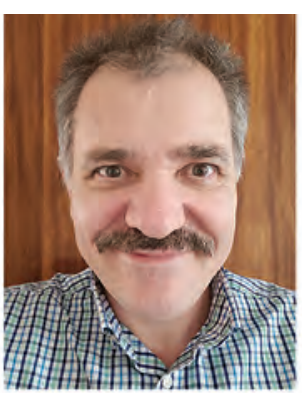

Pantelimon Stănică

\section{Credits}

Figure 1 was reprinted from [10] with permission.

Photos of Lilya Budaghyan and Pantelimon Stănică are courtesy of the authors. 


\section{The SASTRA Ramanujan Prize: Its Origins and Its Winners}

\section{Krishnaswami Alladi}

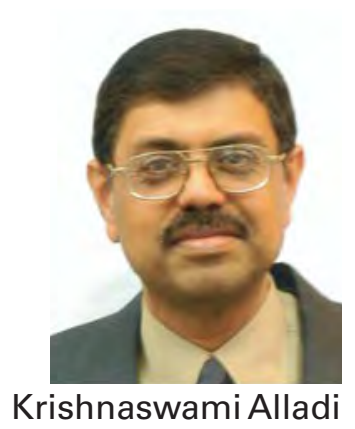

The SASTRA Ramanujan Prize is a $\$ 10,000$ annual award given to mathematicians not exceeding the age of 32 for path-breaking contributions in areas influenced by the genius Srinivasa Ramanujan. The prize has been unusually effective in recognizing at an early stage in their careers extremely gifted mathematicians who have gone on to accomplish even greater things in mathematics and be awarded prizes with a hallowed tradition such as the Fields Medal. This is due to the enthusiastic support from leading mathematicians around the world and the caliber of the winners. The age limit of 32 is because Ramanujan lived only for 32 years, and in that brief lifespan made revolutionary contributions; so the challenge for the prize candidates is to show what they have achieved in that same time frame! The way the prize was conceived and launched is an incredible story, which I will relate here. I will then briefly describe some major aspects of the work of the winners.

\section{The Origins}

The district of Tanjore (=Tanjavur) in the state of Tamil Nadu in South India has been a seat of culture for several centuries. Tanjore has produced some of greatest composers and performers of South Indian classical music. Tanjore is well known for art in various forms and has a great concentration of Hindu temples. It is in this region steeped in culture that Ramanujan was born on December 22, 1887, in the town of Kumbakonam, where he lived until he completed high school.

Krishnaswami Alladi is professor of mathematics at the University of Florida, Gainesville. His email address is a11adi k@uf1.edu.

For permission to reprint this article, please contact: reprint -permission@ams.org.

DOI: http://dx.doi.org/10.1090/noti1765
During the second half of the twentieth century, Ramanujan's humble home in Kumbakonam, from where a thousand theorems emerged, was in a dilapidated condition. Even though in India many events and programs were held regularly in Ramanujan's memory, including the grand Ramanujan Centenary celebrations in December 1987, nothing was done for the renovation of this historic home. One of the most significant developments in the worldwide effort to preserve and honor the legacy of Ramanujan is the purchase in 2003 of Ramanujan's home in Kumbakonam by SASTRA University to maintain it as a museum. This purchase had far-reaching consequences because it led to the involvement of a university in the preservation of Ramanujan's legacy for posterity.

The Shanmugha Arts, Science, Technology and Research Academy (SASTRA), is a private university in the town of Tanjore after which the district is named. SASTRA was founded in 1984 and has grown by leaps and bounds. Admission is very competitive, and SASTRA has succeeded in attracting some of the brightest students in India.

SASTRA has renovated Ramanujan's home beautifully without altering its structure or design. The only modification was to add a bust of Ramanujan in the living room. In connection with the purchase of Ramanujan's home, SASTRA University opened a branch campus in Kumbakonam in 2003, called the Srinivasa Ramanujan Centre. SASTRA has a museum there in which several important letters, photographs, and documents related to Ramanujan are displayed. A visit to Kumbakonam just to see Ramanujan's home and this museum will be worthwhile and inspiring.

One afternoon in September 2003, I received a phone call from S. Swaminathan, then a graduate student at the University of Virginia (now a dean at SASTRA). He introduced himself as the son of Vice-Chancellor R. Sethur- 
aman of SASTRA University, gave me some background about SASTRA, and said that to mark the occasion of the purchase of Ramanujan's home, SASTRA would be conducting an international conference that year in December at the Kumbakonam campus. The President of India, Dr. Abdul Kalam, had agreed to inaugurate this conference on December 20, 2003 and declare Ramanujan's home as a national treasure. Swaminathan invited me to bring a team of mathematicians from abroad to the conference, and said that SASTRA would cover all their expenses including international travel. I felt that what I heard over the phone was incredible because international airfare is rarely paid for mathematical conferences in India. But on checking I realized that something important was taking place. So I called George Andrews of Pennsylvania State University, the world's foremost authority in the theory of partitions and on Ramanujan's work, told him about SASTRA, and requested that he come to the conference. He said that, based on my assurance, he would come. In fact Andrews gave the opening lecture of the conference as well as the concluding Ramanujan Commemoration Lecture on December 22, Ramanujan's birthday. At the valedictory function, the participants suggested that SASTRA should conduct conferences annually around Ramanujan's birthday on different areas of mathematics influenced by Ramanujan. Dean Vaidhyasubramaniam of SASTRA agreed to this suggestion and invited me to help organize these annual conferences. I have done this annually since 2003, and so have also had the pleasure of being in Ramanujan's hometown each year in December thus making it an annual pilgrimage for me (see article 25 in [1], pp. 153-160).

For the second SASTRA Ramanujan Conference in 2004, the Vice-Chancellor invited me to inaugurate it and also deliver the concluding Ramanujan Commemoration lec-

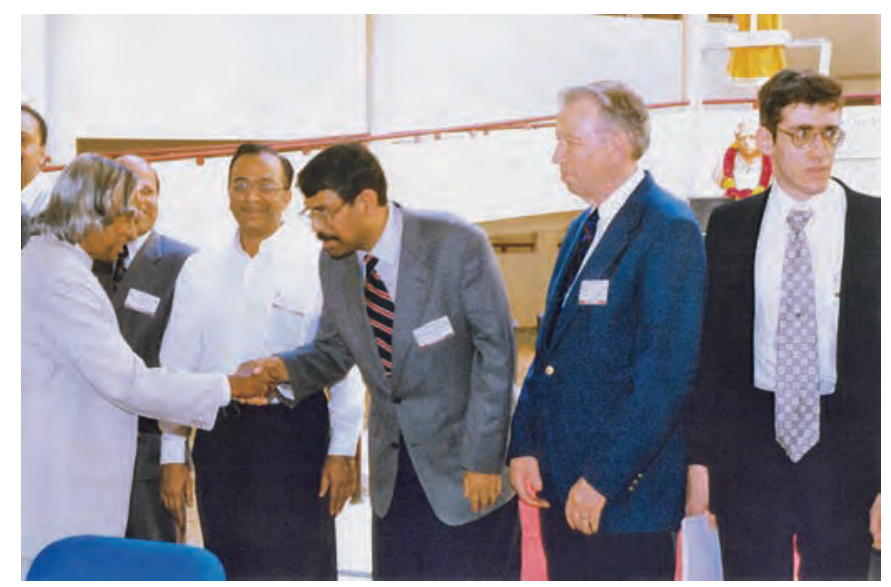

Figure 1. Krishna Alladi being introduced to the President of India, Dr. Abdul Kalam, during the First Ramanujan Conference at SASTRA University on December 20, 2003. To the left of Krishna are George Andrews (Penn State) and Noam Elkies (Harvard). ture. I was honored to get such an invitation, and I accepted it happily.

Conference inaugurations in India are usually elaborate and grand ceremonies starting with the traditional "lighting of the lamp" by the Chief Guest, with the lamp representing enlightenment through knowledge. There was a large gathering in the auditorium, and as we walked up to the stage to be seated before the official start of the ceremony, the Vice-Chancellor whispered to me and said, "I wish to give $\$ 10,000$ annually for a worthy cause in the name of Ramanujan. You please decide how it should be used and announce it in your inaugural speech." I was pleasantly shocked by this and said, "This is very generous of you, but are you asking me to announce this in the next few minutes without consulting anybody?" He said, "Yes, consulting people, or going through a Committee, will cause unnecessary delays, and so I want you to come to decision right now and announce it." Fortunately, my inaugural speech was preceded by the lamp lighting ceremony and the customary elaborate welcome of the audience and the introduction of the Chief Guest (me) as is common in India, and so I had at least fifteen minutes to think about it as all this was going on! When I got up to speak, I mentioned this very generous offer from the Vice-Chancellor, and suggested that a SASTRA Ramanujan Prize be created and that $\$ 10,000$ be given to a mathematician not exceeding the age of 32 for outstanding contributions to areas influenced by Ramanujan. I pointed out that the Fields Medal is given only to those under 40 years of age, and the SASTRA Prize would be given to even younger mathematicians, some of whom may later win the Fields Medal or other well-established major prizes. The Vice-Chancellor liked this suggestion very much, and at the conclusion of the inaugural ceremony, in thanking me, he confirmed that the prize would be launched the next year. He then turned towards me and said, "I request you to be the Chair of the Prize Committee." So that is how the SASTRA Ramanujan Prize was conceived and launched, and how I got involved with the Prize. All this happened within the span of an hour during the inauguration.

Upon return to the United States, I called George Andrews and told him this incredible story of the launch of the prize and said that for this to be a success, I would need the support of the mathematical community. In particular, I wanted his presence on the Committee for the first year. He was surprised and happy to hear this and agreed to serve on the Prize Committee. Over the years, eminent mathematicians have supported the prize by either serving on the Prize Committee, making nominations, or writing letters evaluating the work of the nominees. The prize is given annually at SASTRA University, Kumbakonam, during an international conference held around Ramanujan's birthday. 


\section{The winners}

The 2005 Prizes: Manjul Bhargava of Princeton University and Kannan Soundararajan of the University of Michigan came out on top as the strongest young mathematicians in the areas of algebraic number theory and analytic number theory, respectively, and were equally ranked 1 by the Committee.

One of the pioneering discoveries of Gauss was the composition law for binary quadratic forms. Introducing several new and unexpected ideas, Bhargava broke an impasse since the time of Gauss, and established in his $2001 \mathrm{PhD}$ thesis at Princeton University composition laws for higher degree forms. Bhargava applied these to solve new cases of one of the fundamental questions of number theory, that of the asymptotic enumeration of number fields of a given degree. He published the results in his thesis in a series of papers in the Annals of Mathematics. Bhargava's lecture at SASTRA University upon accepting the prize was on a different topic; he announced his joint work with Jonathan Hanke on the solution of the problem of determining all universal quadratic forms-a problem whose origin can be traced back to Ramanujan.

Kannan Soundararajan had made spectacular contributions to analytic number theory, most notably pertaining to the Riemann zeta function and Dirichlet $L$-functionsespecially on the distribution and location of their zeros. He also established deep results in random matrix theory, which has fundamental connections with prime number theory. In his PhD thesis at Princeton University, he showed that $7 / 8$-ths of the quadratic $L$-functions have no zeros at

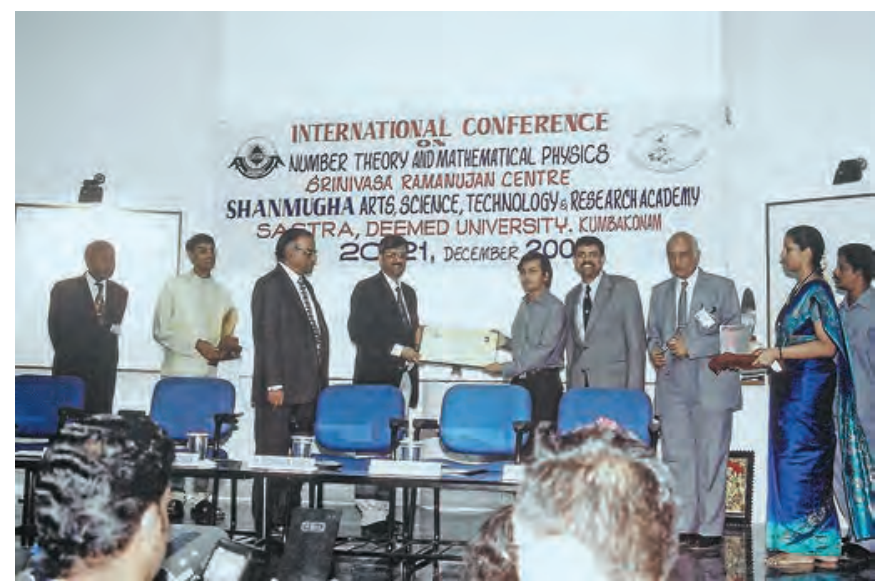

Figure 2. Kannan Soundararajan (then at Michigan) receiving the first SASTRA Ramanujan Prize on December 20, 2005 from Dr. Arabinda Mitra, Director, Department of Science andTechnology, India. (Second from Left) Manjul Bhargava (Princeton) who had received the same prize a few minutes earlier, looks on. Next to Mitra is SASTRA Vice-Chancellor R. Sethuraman, and next to Soundararajan is Krishna Alladi. the critical point $s=1 / 2$, which provided strong evidence for a certain conjecture of Chowla. Along with Brian Conrey, he had shown that a positive proportion of Dirichlet $L$-functions have no zeros on the real axis within the critical strip.

The Prize Committee felt that both candidates deserved the full award. The SASTRA Vice-Chancellor generously agreed to the recommendation of the Committee that Bhargava and Soundararajan be awarded two full prizes, and that it should not be split. Thus the SASTRA Ramanujan Prize could not have had a better start (see Ken Ono's article [3] on the award of the first SASTRA Ramanujan Prizes, and mine ([1], p. 161-166) as well).

Bhargava continued producing fundamental work after receiving the SASTRA Ramanujan Prize, especially related to the average rank of elliptic curves. He was recognized with the AMS Cole Prize in 2008 and the Fields Medal in 2014.

When Soundararajan received the SASTRA Prize, he was a tenured faculty member at Michigan, and in the next year, was appointed full professor at Stanford. Subsequently, he was recognized with the Infosys Prize and the Ostrowski Prize, both in 2011.

The 2006 Prize: Thirty-one-year-old Terence Tao of UCLA had made far-reaching contributions to diverse areas of mathematics such as number theory, harmonic analysis, partial differential equations, and ergodic theory. He was widely regarded as one of the most influential mathematicians of our time.

One of Tao's most notable contributions was for the Kakeya Problem in higher dimensions. One aspect of the problem is to determine the fractal dimension of a set obtained by rotating a needle in $n$-dimensional space. In joint work with Nets Katz, Isabella Laba, and others, he improved all previously known estimates for the fractal dimension with ingenious combinatorial ideas. Another of Tao's seminal contributions was his joint work with Ben Green on long arithmetic progressions of prime numbers. One of the deepest results in this area is due to the Hungarian mathematician Szemerédi who showed that any set of positive density will have arbitrarily long arithmetic progressions. Subsequently Tim Gowers of Cambridge University gave a very different proof of Szemerédi's theorem. This result of Szemerédi does not apply to the set of primes, which is of zero density. By combining the ideas of Gowers along with tools from ergodic theory, Green and Tao proved the sensational result that there are arbitrarily long arithmetic progressions of primes.

Yet another fundamental contribution of Tao concerns the sum-product problem due to Paul Erdős and Szemerédi. Roughly speaking, this problem states that either the sum set or the product set of a set of $N$ numbers must be large. Tao was the first to recognize the significance of this problem in combinatorial number theory and harmonic analysis. In collaboration with Nets Katz and Jean 


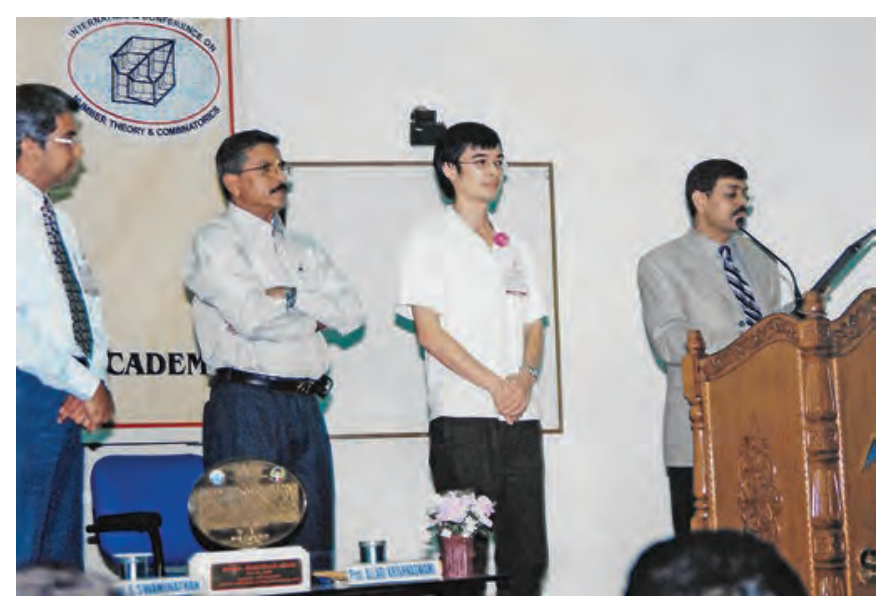

Figure 3. Krishna Alladi reads the citation before the 2006 SASTRA Ramanujan Prize is presented to TerenceTao (standing, middle) of UCLA.

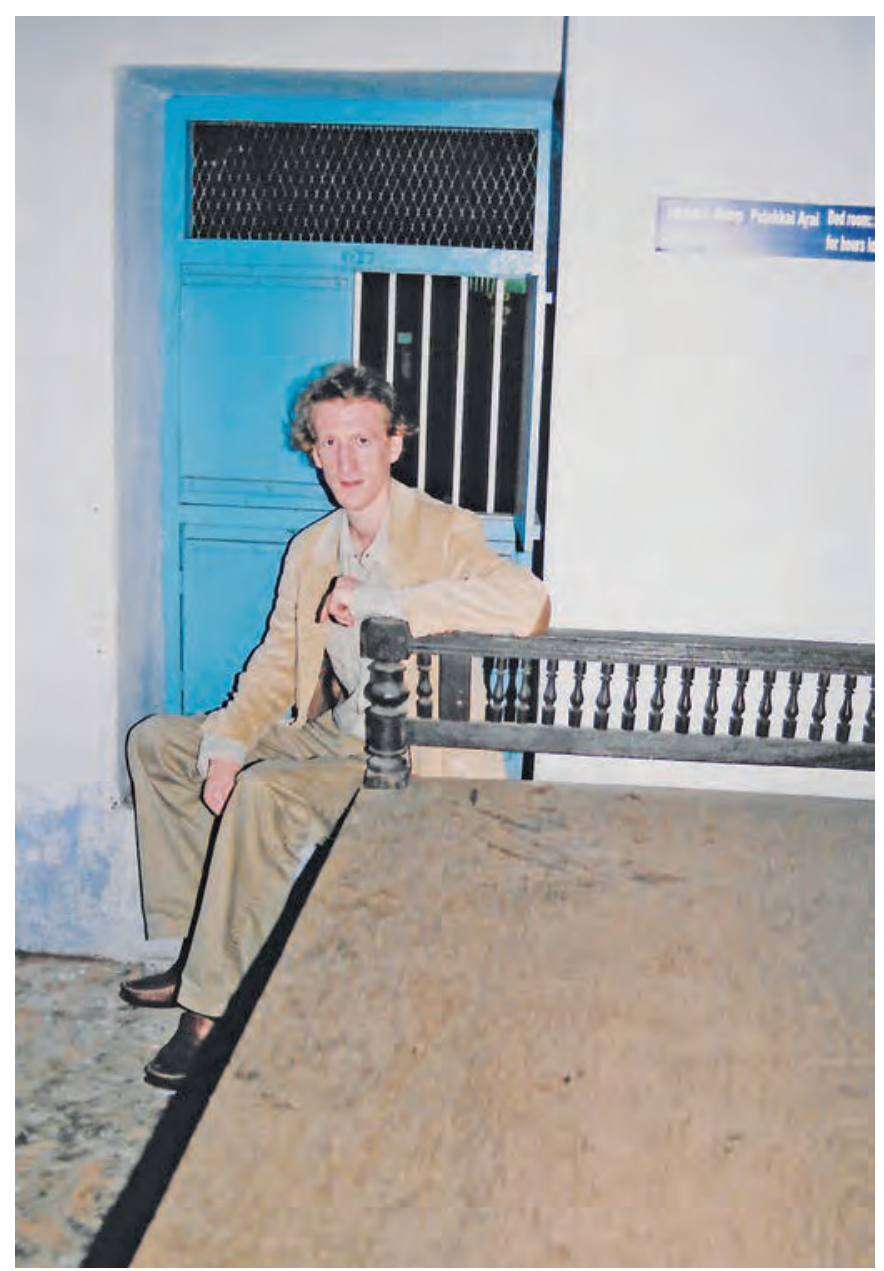

Figure 4. 2007 SASTRA Prize Winner Ben Green (then at Cambridge University) seated on the windowsill of the bedroom at Ramanujan's home. The cot there was the only one for Ramanujan's family. As a boy, Ramanujan used to sit on the windowsill and do his "sums" watching the passers-by on the street.
Bourgain, Tao made important generalizations that led to breakthroughs in harmonic analysis and number theory.

Honors have come to him in a steady stream. In 2006 Tao was awarded both the Fields Medal and the SASTRA Ramanujan Prize. More recently, he received the 2014 Breakthrough Prize. He currently holds the James and Carol Collins Chair at UCLA.

The 2007 Prize: Ben Green of Cambridge University had made phenomenal contributions to several fundamental problems in number theory by himself and in collaboration with Terence Tao. Green's PhD thesis at Cambridge is a collection of several outstanding papers. In one of these papers he solved the Cameron- Erdős conjecture, which is a bound on the number of sum-free sets of positive integers up to a given number $N$. Green had also established an important result that any set of primes with relative positive density would contain infinitely many arithmetic progessions of length 3. It was this paper of Green in the 2005 Annals of Mathematics that caught the attention of Tao and which led to their collaborative and definitive result on arbitrarily long arithmetic progressions among the primes. Subsequently Green and Tao also collaborated in extending the Hardy-Ramanujan-Littlewood Circle Method by bringing in methods from ergodic theory.

In 2005 Green was appointed Hershel Smith Professor at Cambridge University. He was elected Fellow of the Royal Society in 2010 and was awarded the Sylvester Medal in 2014. He is currently Waynflete Professor of Pure Mathematics at Oxford University.

The 2008 Prize: Akshay Venkatesh of Stanford University was creating waves in the mathematical world by making powerful contributions to diverse areas, by himself and with a host of collaborators. His 2006 paper with H. Helfgott contained striking and original ideas, and provided the first non-trivial upper bound for the 3-torsion in class groups of quadratic fields. His joint work with Jordan Ellen-

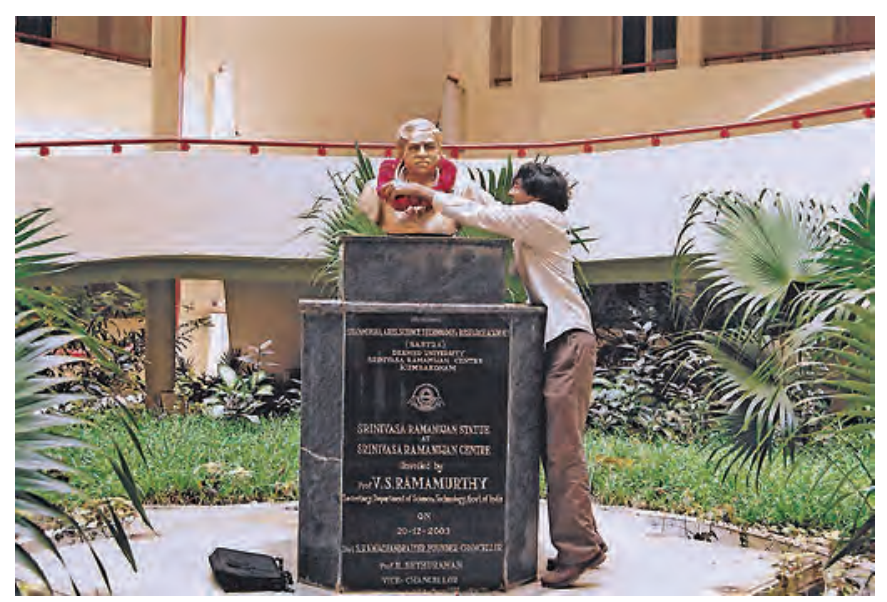

Figure 5. Akshay Venkatesh (Stanford), 2008 SASTRA PrizeWinner, garlanding the statue of Ramanujan at SASTRA University. 
berg on representing integral quadratic forms by quadratic forms had its roots in the work of Ramanujan.

An important and difficult problem in number theory is to asymptotically count number fields according to their discriminant. The case up to degree 5 had been solved by Manjul Bhargava. For large degrees, Ellenberg and Venkatesh provided the first major improvement over bounds in earlier work of Wolfgang Schmidt and thus broke an impasse of thirty years. Also of great importance was Venkatesh's work on sub-convexity of automorphic $L$-functions, and his joint work with E. Lindenstrauss, which settled a famous conjecture of Peter Sarnak concerning locally symmetric spaces.

After the SASTRA Ramanujan Prize, he has been awarded the Infosys Prize in 2016 and the Ostrowski Prize in 2017. In 2018 he was awarded the Fields Medal and appointed to the permanent faculty at the Institute for Advanced Study, Princeton.

The 2009 Prize: The 2009 Prize was awarded to Kathrin Bringmann for work related to Ramanujan's mock theta functions. A good portion of Ramanujan's Lost Notebook, namely, the loose sheets of paper on which he wrote hundreds of formulas shortly before he died in 1920, is devoted to mock theta functions. These are objects that are like the classical theta functions in their shape, but are not modular forms, yet their coefficients can be calculated with a degree of precision comparable to what can be done for functions that can be expressed in terms of theta functions. No one knew the exact relationship between theta functions and mock theta functions, and indeed determining this relationship was one of the tantalizing puzzles of mathematics. Following a lead provided by Sander Zwegers, Kathrin Bringmann and her post-doctoral mentor Ken Ono provided the key to unlock this mystery by showing how mock theta functions are intimately connected with harmonic Maass forms.

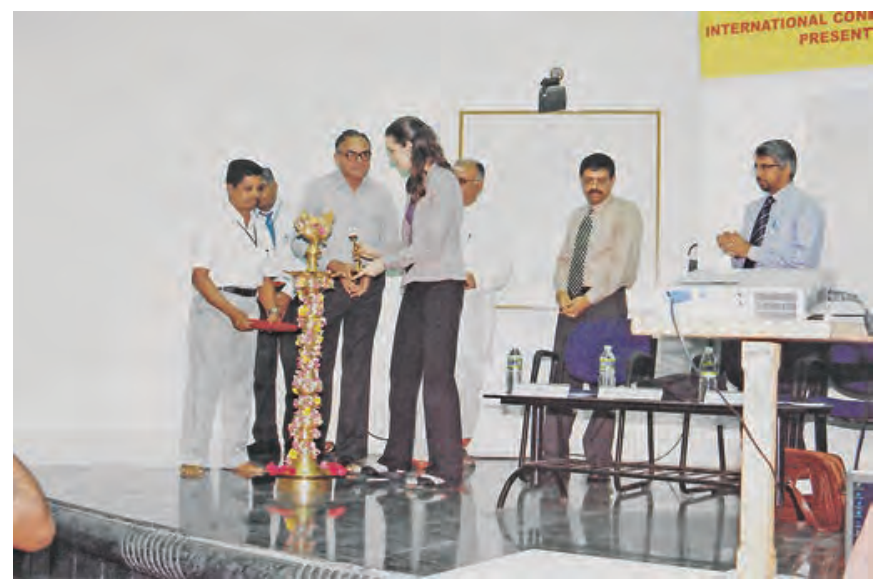

Figure 6. 2009 SASTRA Prize Winner Kathrin Bringmann (University of Cologne) lighting the ceremonial lamp to mark the opening of the Ramanujan Conference.
The 2010 Prize: As a PhD student at Columbia University, Wei Zhang had made seminal contributions by himself and in collaboration with others to a broad range of areas in mathematics including number theory, automorphic forms, trace formulas, $L$-functions, representation theory, and algebraic geometry.

In 1997 Steve Kudla constructed a family of cycles on Shimura varieties, and conjectured that their generating functions are actually Siegel modular forms. The proof of the Kudla conjecture for cycles of codimension 1 is a major theorem of Fields Medalist Richard Borcherds. In his PhD thesis, Zhang established conditionally, among other

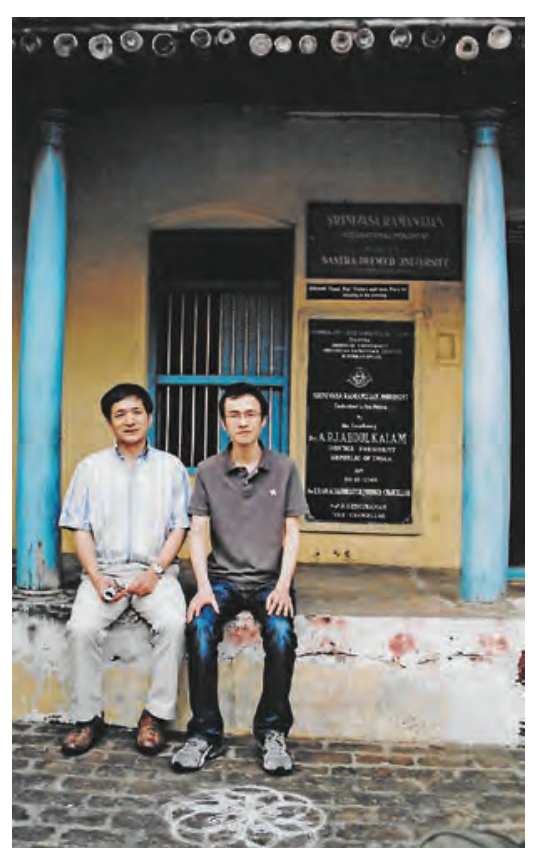

Figure 7. 2010 SASTRA Prize

WinnerWei Zhang (then at Harvard) seated (on the right) along with his former PhD advisor Shouwu Zhang (Columbia) at the entrance to Ramanujan's home in Kumbakonam. things, a generalization of Borcherds' result for higher dimensions and in that process essentially settled the Kudla conjecture. Zhang's thesis opened up major lines of research and led to significant collaboration with Xinyi Yuan and Shouwu Zhang. The three established an arithmetic analogue of a theorem of Waldspurger that connects integral periods to values of $L$-functions. In addition, Wei Zhang by himself had done outstanding work on relative trace formulas, and on Shimura varieties.

When Zhang received the SASTRA Prize, he was Benjamin Pierce Instructor at Harvard. In 2017 he was awarded the New Horizons Prize in Mathematics. He is currently a full professor at Columbia University.

The 2011 Prize: Roman Holowinsky of Ohio State University had made significant contributions at the interface of analytic number theory and the theory of modular forms. Along with Kannan Soundararajan he solved an important case of the famous Quantum Unique Ergodicity (QUE) Conjecture. This was a spectacular achievement.

In 1991, Zeev Rudnick and Peter Sarnak formulated the QUE Conjecture, which in its general form concerns the correspondence principle for quantizations of chaotic systems. One aspect of the problem is to understand how 
waves are influenced by the geometry of their enclosure. Rudnick and Sarnak conjectured that for sufficiently chaotic systems, if the surface has negative curvature, then the high frequency quantum wave functions are uniformly distributed within the domain. The modular domain in number theory is one of the most important examples, and for this case, Holowinsky and Soundararajan solved the holomorphic QUE conjecture.

The manner in which this solution came about is amazing. By a study of Hecke eigenvalues and an ingenious application of the sieve, Holowinsky obtained critical estimates for shifted convolution sums and this almost settled the holomorphic QUE conjecture for the modular domain except in certain cases where the corresponding $L$-functions behave abnormally. Simultaneously, Soundararajan approached the problem from an entirely different direction, was able to confirm the conjecture in several cases, and noticed that the exceptional cases not fitting Holowinsky's approach were covered by his techniques. Thus by combining the approaches of Holowinsky and Soundararajan, the holomorphic QUE Conjecture was fully resolved in the modular case.

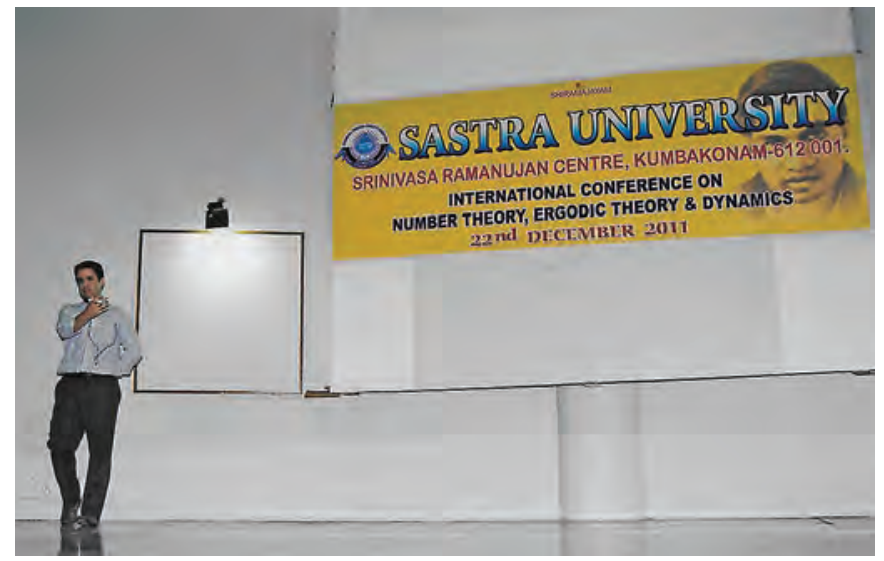

Figure 8. 2011 SASTRA Prize Winner Roman

Holowinsky (Ohio State) delivering the Ramanujan

Commemoration Lecture on December 22,

Ramanujan's birthday.

The 2012 Prize: Zhiwei Yun of Stanford University had made fundamental contributions to areas that lie at the interface of representation theory, algebraic geometry, and number theory. Yun's PhD thesis on global Springer theory at Princeton University opened up new vistas in the Langlands program. Springer theory is the study of Weyl group actions on the cohomology of certain subvarieties of the flag manifold called Springer fibers. Yun's global Springer theory deals with Hitchin fibers instead of Springer fibers, which he used to determine the actions of affine Weyl groups on cohomology.

Bao-Châu Ngô was awarded the 2010 Fields Medal for his proof of the Fundamental Lemma in the Langlands
Program. Yun made a breakthrough in the study of the Fundamental Lemma formulated by Jacquet and Rallis in their program of proving the Gross-Prasad conjecture on relative trace formulas. Yun's understanding of Hitchin fibrations enabled him to reduce the Jacquet-Rallis fundamental lemma to a cohomological property of the Hitchin fibration. In addition, Yun's work on the uniform construction of motives with exceptional Galois groups is considered to be very fundamental.

Since 2012 was the 125th Birth Anniversary of Ramanujan, Yun was awarded the SASTRA Prize in India's capital New Delhi, at a conference organized by the National Board for Higher Mathematics (of India), and co-sponsored by SASTRA University and Delhi University. That was the only year when this prize was not awarded in Kumbakonam.

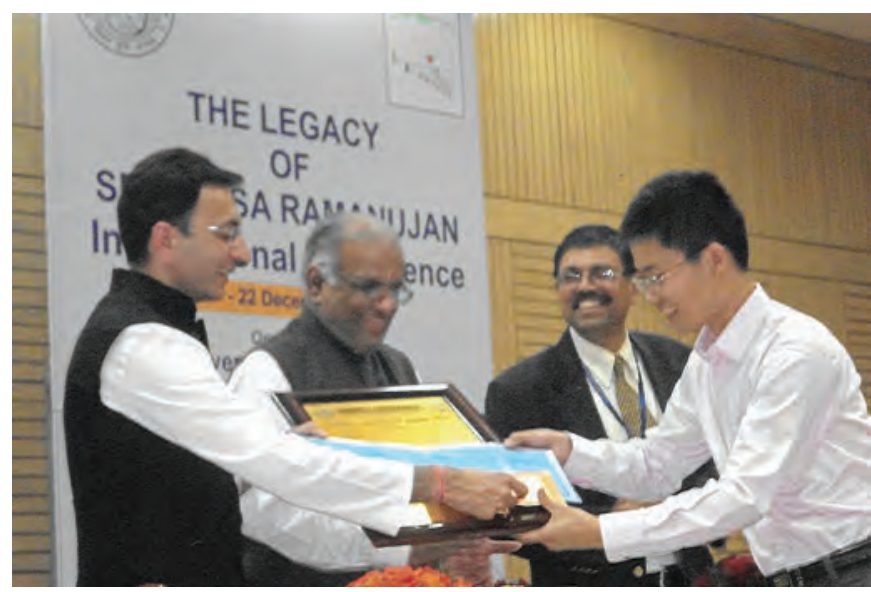

Figure 9. Zhiwei Yun (MIT and Stanford) receiving the 2012 SASTRA Ramanujan Prize from Minister of State Jitin Prasada at the University of Delhi on December 22. SASTRA Vice-Chancellor Sethuraman and Krishna Alladi are looking on.

When Yun received the SASTRA Prize, he had just been appointed to the permanent faculty at Stanford University after having completed his term as Moore Instructor at MIT. In 2018 he was a recipient of the New Horizons in Mathematics Prize.

The 2013 Prize: Peter Scholze of the University of Bonn had made revolutionary contributions to arithmetic algebraic geometry and the theory of automorphic forms. Already in his Masters thesis at Bonn, Scholze gave a new proof of the Local Langlands Conjecture for general linear groups based on a novel approach to calculate the zeta function of certain algebraic varieties. While this was groundbreaking, his $\mathrm{PhD}$ thesis at Bonn was a much bigger breakthrough: he developed a new $p$-adic machine called perfectoid spaces and used it brilliantly to prove a significant part of the weighted monodromy conjecture due to Deligne, thereby breaking an impasse of more than 30 years. Scholze extended his theory of perfectoid spaces 


\section{COMMUNICATION}

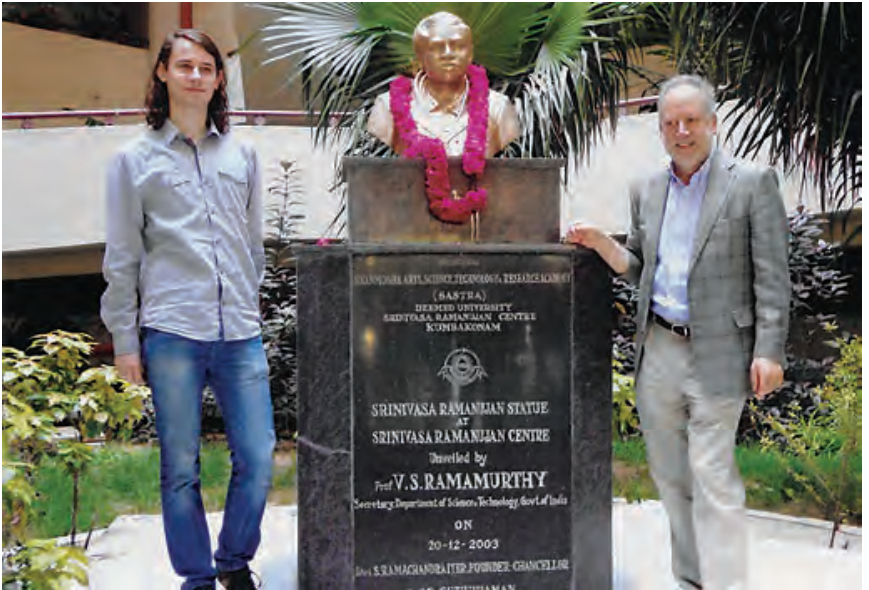

Figure 10. ( $L$ to R) 2013 SASTRA Prize Winner Peter Scholze (University of Bonn) and his former PhD Advisor Michael Rappoport (Bonn) standing next to the statue of Ramanujan at SASTRA University.

to develop a Hodge theory for rigid analytic spaces over $p$-adic ground fields, generalizing a theory due to Faltings for algebraic varieties.

Scholze was the youngest winner of the SASTRA Prize at the age of 25. Following that, he has been recognized with the AMS Cole Prize (2015), the Fermat Prize (2015), the Ostrowski Prize (2015), the Leibniz Prize (2016), and the Fields Medal (2018).

The 2014 Prize: James Maynard of Oxford University, England, and of the University of Montreal, had made outstanding contributions to some of the most famous problems on prime numbers. He obtained the strongest results at the time on the celebrated prime twins conjecture by showing that the gap between consecutive primes does not exceed 600 infinitely often. Not only did he significantly improve the path-breaking work of Goldston, Pintz, Ylldırım, and Zhang, but he achieved it with ingenious methods that were simpler than those used by others.

A generalization of the prime twins conjecture is the prime $k$-tuples conjecture, which states that an admissible collection of $k$ linear functions will simultaneously take $k$ prime values infinitely often. In the last one hundred years, several partial results towards the $k$-tuples conjecture were obtained by replacing prime values with "almost primes," namely numbers with a bounded number of prime factors. Another major achievement of Maynard was to significantly improve on the work of earlier researchers on $k$-tuples of almost primes.

Just before receiving the SASTRA Prize, Maynard announced the solution to the famous $\$ 10,000$ problem of Erdős on large gaps between primes. This was simultaneously announced by Kevin Ford, Ben Green, Sergei Konyagin, and Terence Tao, but Maynard's method was different and simpler. Maynard's results and methods have led to a resurgence of activity worldwide in prime number theory.

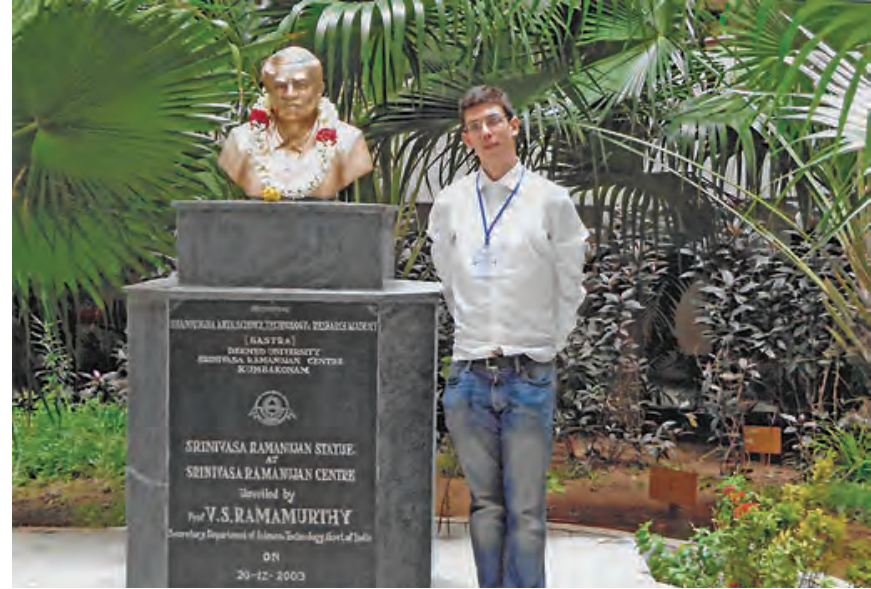

Figure 11. James Maynard (Oxford University), the 2014 SASTRA Prize Winner, standing next to the Ramanujan statue at SASTRA University.

After the SASTRA Prize, he received the Whitehead Prize in 2015 and the EMS Prize in 2016. He is currently a professor at Magdalen College, Oxford University.

The 2015 Prize: Jacob Tsimerman of the University of Toronto had made deep and original contributions to diverse parts of number theory, most notably to the André-Oort Conjecture, which states that special subsets of Shimura varieties that are obtained as Zariski closures of special points are finite unions of Shimura varieties. Shimura varieties are special algebraic varieties (such as moduli spaces of abelian varieties) that arise as quotients of suitable complex domains by arithmetic groups. Yves André initially stated this conjecture for one-dimensional sub-varieties, and subsequently Frans Oort proposed that

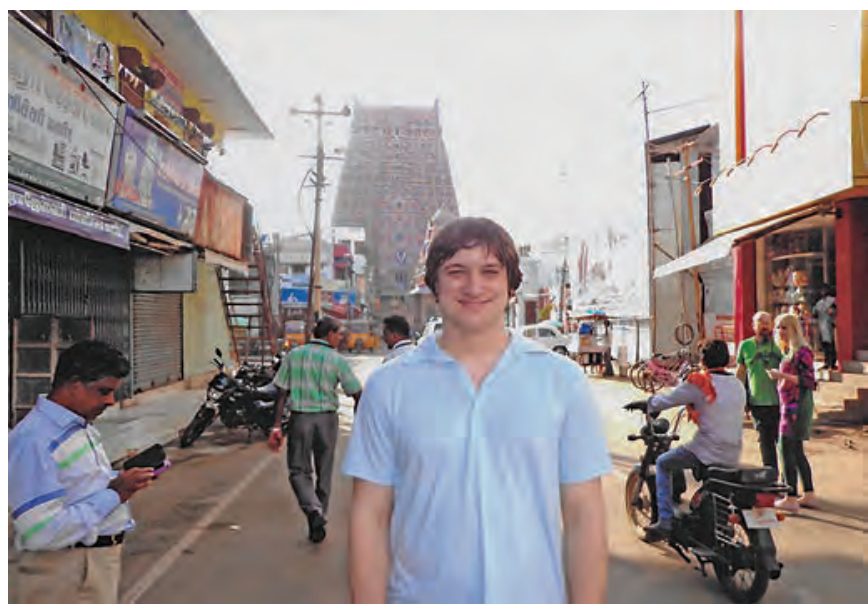

Figure 12. 2015 SASTRA Prize Winner Jacob

Tsimerman (University of Toronto) standing outside Ramanujan's home. The majestic Gopuram (entrance tower) of the Sarangapani Temple, where Ramanujan and family regularly worshipped, is seen in the background. 
it should hold more generally. A major achievement of Tsimerman in his Princeton PhD thesis of 2011 was to obtain certain unconditional bounds up to dimension 6 . Another important result in his thesis was to answer in the affirmative a question of Nick Katz and Oort whether there exists an abelian variety over the set of all algebraic numbers that is not isogenous to the Jacobian of a stable algebraic curve over the algebraic numbers.

Just before receiving the SASTRA Prize, Tsimerman gave a proof of the André-Oort Conjecture for the moduli spaces of principally polarized abelian varieties of any dimension-a result that was sought for a long time.

The 2016 Prize: This was shared by Kaisa Matomäki of the University of Turku, Finland, and Maksym Radziwill of McGill University, Canada, and Rutgers University. Their revolutionary collaborative work on multiplicative functions in short intervals shocked the mathematical community by going well beyond what could be proved

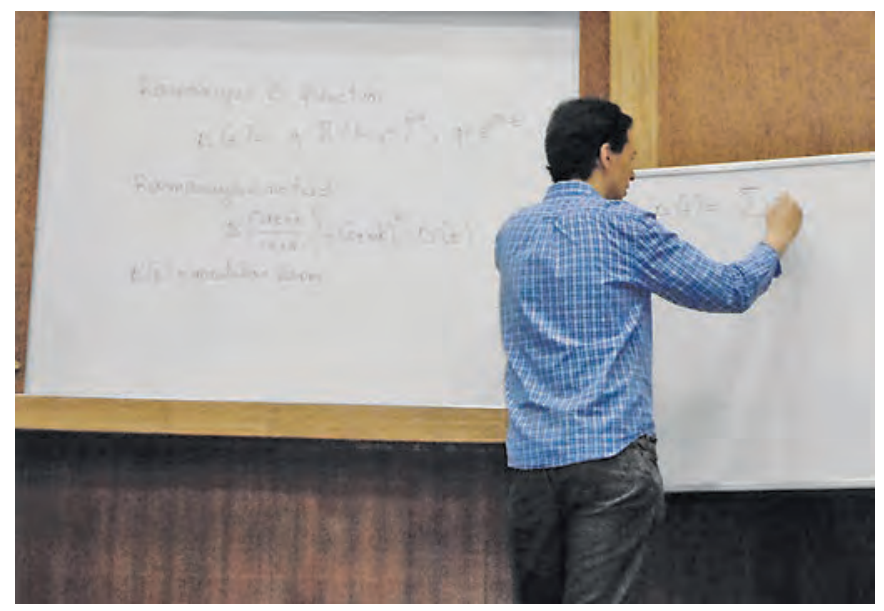

Figure 13. Maksym Radziwill (McGill University) giving the Ramanujan Commemmoration Lecture on his joint work with Kaisa Matomäki with whom he shared the 2016 SASTRA Prize.

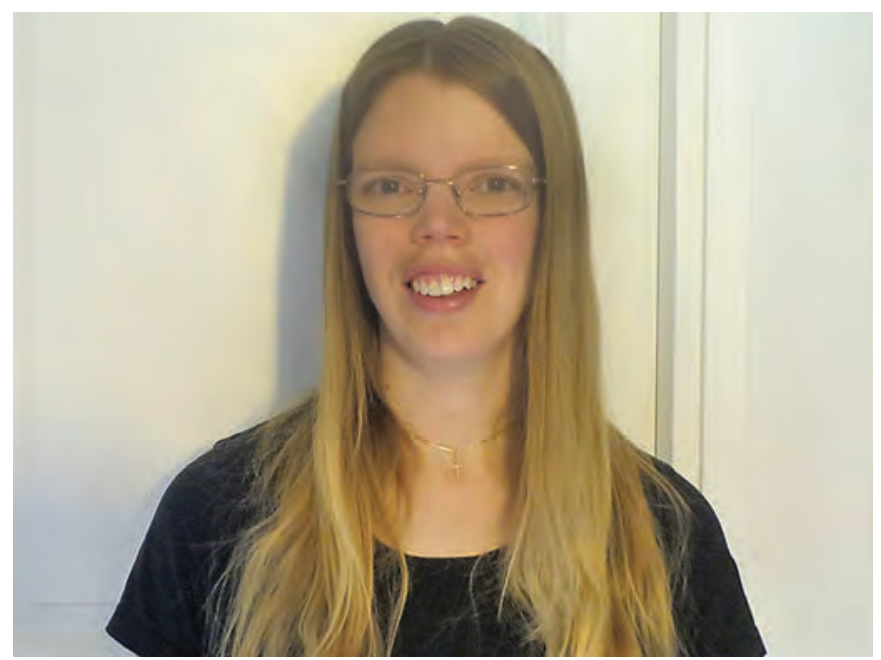

Figure 14. Kaisa Matomäki. previously even assuming the Riemann Hypothesis, and opened the door for a series of breakthroughs on some notoriously difficult questions such as the Erdős discrepancy problem and Chowla's conjecture, previously believed to be well beyond reach. Their stunning work on multiplicative functions in short intervals is best illustrated in terms of the Liouville lambda function, which takes value 1 when an integer has an even number of prime factors (counted with multiplicity), and value -1 at an integer with an odd number of prime factors. The statement that the lambda function takes values 1 and - 1 with asymptotically equal frequency is equivalent to the Prime Number Theorem; more refined statements on the relative error in this equal frequency are related to the Riemann Hypothesis. Such equal distribution results were known also for short intervals, namely intervals $[x, x+h]$, where $h$ is a fractional power $(<1)$ of $x$. The Riemann Hypothesis implies that powers of $h$ larger than $1 / 2$ will work. Instead of every interval of length $h$, if we require only "almost all" intervals of length $h$, it was known that $h$ could be made as small as the $1 / 6$ th power of $x$, and as small as a power of $\log x$ by assuming the Riemann Hypothesis. Matomäki and Radziwill shocked the world by showing unconditionally that equal frequency holds almost always as long as $h$ tends to infinity with $x$.

Sarvadaman Chowla conjectured that if any $k$ collection of values of 1 and -1 are given in any order, then the lambda function will take that sequence of values at $k$ consecutive integers with asymptotic frequency $1 / 2^{k}$. This conjecture is yet unsolved. In 2016, Matomäki, Radziwill, and Terence Tao proved that when $k=3$, each of the eight sign choices occur with positive proportion.

The 2017 Prize: Maryna Viazovska of the Swiss Federal Institute of Technology, Lausanne, was awarded the 2017 SASTRA Prize for her stunning solution in dimension 8 of the sphere-packing problem, and for her equally impressive joint work with Henry Cohn, Abhinav Kumar, Stephen D. Miller, and Danylo Radchenko, resolving the sphere-packing problem in dimension 24 , by building upon her fundamental ideas in dimension 8.

The sphere-packing problem has a long and illustrious history. Johannes Kepler asked for the optimal way to stack cannon balls (of uniform radius) and conjectured a configuration, but could not prove it. This Kepler Conjecture was resolved by Thomas Hales in 1998 by combining ingenious geometric optimization arguments with machine calculations. The sphere-packing problem in higher dimensions remained open.

In dimension 8 there is $E_{8}$, an exceptional Lie group with a root lattice of rank 8 , and in dimension 24 there is the Leech lattice. This gave some hope that the sphere-packing problem could be solved in dimensions 8 and 24. Noam Elkies and Henry Cohn made significant progress and conjectured the existence of certain magic auxiliary functions in dimensions 8 and 16, which, if determined, would resolve 
the conjecture in these dimensions. Viazovska produced these functions in dimension 8 by ingenious use of modular forms. Her proof is remarkably simple. Within a span of week, working at a furious pace, by extending the ideas in dimension 8, the sphere-packing problem in dimension 24 was resolved by Cohn, Kumar, Miller, Radchenko, and Viazovska.

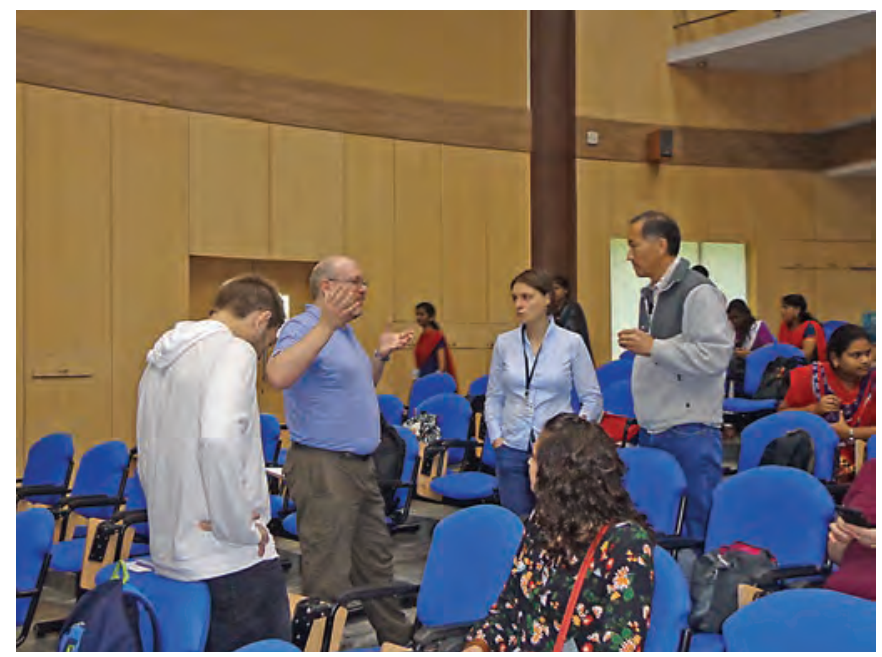

Figure 15. (L to R) Henry Cohn (Microsoft Research) discussing with 2017 SASTRA PrizeWinner Maryna Viazovska (EPF Lausanne) and Ken Ono (Emory)

In 2018 Viazovska was also awarded the New Horizons in Mathematics Prize.

Shaping the development of mathematics: The Fields Medals, with an age limit of 40 for the winners, were instituted with two lofty goals in mind: (i) to recognize pioneering work by brilliant young mathematicians, and (ii) to encourage these young researchers to continue to influence the growth of mathematics. The Fields Medalists have lived up to these great expectations. The SASTRA Ramanujan Prize has a more stringent age limit of 32, and so has recognized brilliant mathematicians even earlier in their careers. It is no exaggeration to say that the winners of the SASTRA Ramanujan Prizes have also shaped the development of mainstream mathematics and will continue to do so in the years ahead; the fact that the SASTRA laureates have subsequently won major prizes is a testimony to this. Indeed, two of the four 2018 Fields Medalists are former SASTRA Ramanujan Prize Winners: Akshay Venkatesh and Peter Scholze! Similarly, three of the four 2018 New Horizons in Mathematics Prize winners are former SASTRA awardees: Wei Zhang, Zhiwei Yun, and Maryna Viazovska.

\section{References}

1) Alladi K. Ramanujan's place in the world of mathematics essays providing a comparative study, Springer, New Delhi (2012), 177 pp.

2) Alladi K, "Ramanujan's thriving legacy," in Srinivasa Ramanujan going strong at 125, Parts I and II, Notices Amer. Math. Soc., I: 59 (2012), 1522-1537; II: 60 (2013), 10-22. MR3027107 and MR3052461

3) Ono K, Honoring a gift from Kumbakonam, Notices Amer. Math. Soc., June-July 2006, 641-651. MR2235326

\section{Credits}

Figure 9 is courtesy of SASTRA.

Figure 14 is by Pekka Matomäki.

All other article photos and author photo are courtesy of the Krishnaswami Alladi.

The 2018 SASTRA Ramanujan Prize is shared by Yifeng Liu (Yale University) and Jack Thorne (Cambridge University) for spectacular contributions to algebraic geometry, automorphic representations, and number theory.

See p.113 of this issue for the Math People announcement about the 2018 SASTRA Prize to Liu and Thorne. 


\section{FAN CHINA
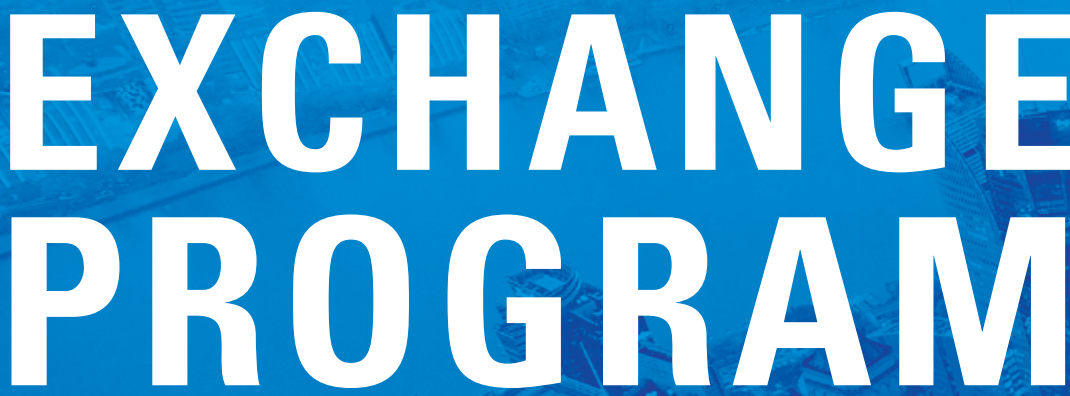

- Gives eminent mathematicians from the US and Canada an opportunity to travel to China and interact with fellow researchers in the mathematical sciences community.

- Allows Chinese scientists in the early stages of their careers to come to the US and Canada for collaborative opportunities.

Applications received before March 15 will be considered for the following academic year.

For more information on the Fan China Exchange Program and application process see www.ams.org/china-exchange or contact the AMS Professional Programs Department:

TELEPHONE: 800.321.4267, ext. 4105 (US \& Canada) 401.455 .4105 (worldwide)

EMAIL: chinaexchange@ams.org.
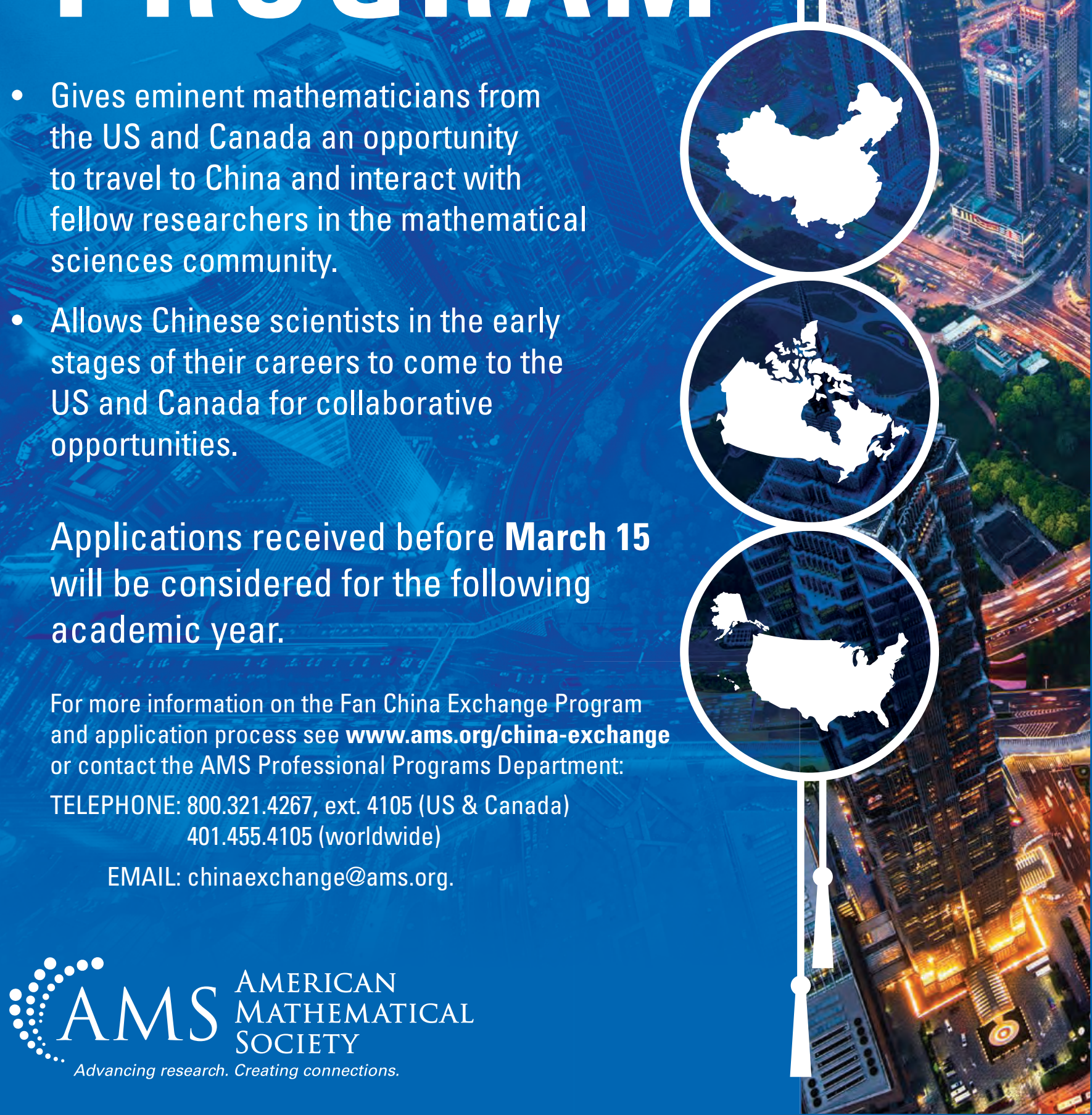


\section{BOOK REVIEW}

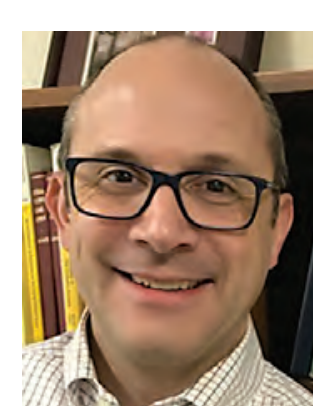

\section{A Comprehensive Course in Analysis}

\section{A review by William T. Ross}

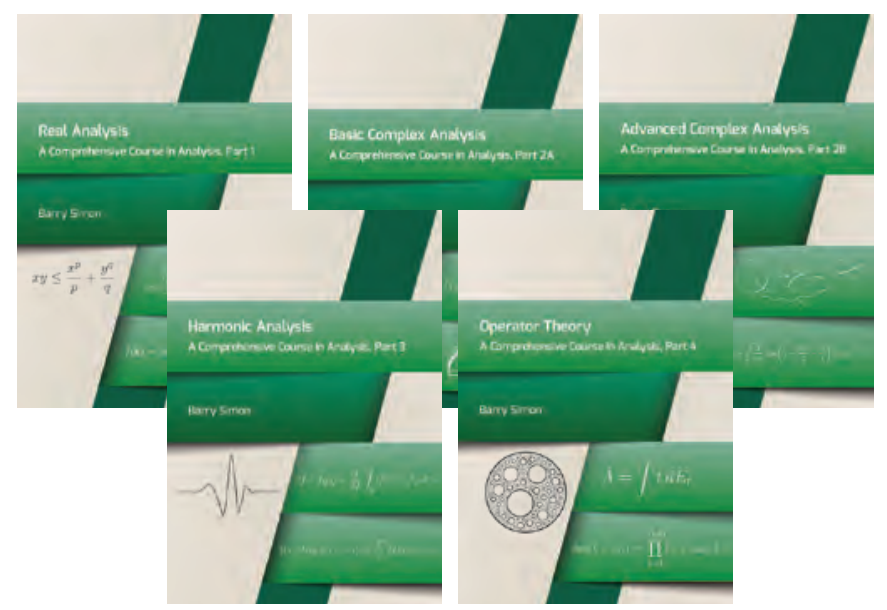

A Comprehensive Course in Analysis (5-volume set)

Barry Simon

AMS, 2015

ISBN: 978-1470410988

On my top bookshelf is a set of analysis books I often reference to make sure I'm using a theorem correctly or perhaps to review a long-forgotten fact. I have my favorite books for real analysis (Royden, Rudin), functional analysis (Conway, Rudin), operator theory (Akhiezer and Glazman), complex analysis (Alhfors, Rudin, Markushevich), harmonic analysis and function spaces (Duren, Garnett, Koosis), and, of

William T. Ross is the Roger Francis and Mary Saunders Richardson Chair in mathematics at the University of Richmond. His email address is wross@richmond.edu.

Communicated by Notices Book Review Editor Stephan Ramon Garcia. For permission to reprint this article, please contact: reprint -permission@ams.org.

DOI: http://dx.doi.org/10.1090/noti1769 course, Kelly's book to shore up my set theory and topology. All of these authors have different writing styles and levels of details for their proofs. When I need to focus further, I refer to my second shelf of books to dig a bit deeper on more specialized knowledge such as Bergman spaces, Dirichlet spaces, Toeplitz operators, Hankel operators, and composition operators.

To help us trim down our burgeoning book collections, there is a comprehensive collection of all things analysis with Barry Simon's A Comprehensive Course in Analysis. This course is a five-volume tour de force by a preeminent mathematical physicist and educator who cares deeply about how analysis is presented to graduate students and working analysts. This series of books covers the major parts of analysis and can be selectively used as parts of courses as well as a reference-both mathematical and historical. In fact, this undertaking is so enormous, that Simon includes a separate 68-page guide (The Ultimate Companion....) for the volumes.

The analysis community is at times so specialized, and maybe too focused on their own work, that at conferences, we often talk past each other rather than to each other. We often lack a set of common ideas and knowledge base. So, in a way, a set of books like Simon's reminds us of what should be the corpus of analysis that we all should know. These books also allow more experienced researchers who are perhaps focused on the minutia of their own work, to expand their analysis foundations so they can, in the future, appreciate a department colloquium talk not directly in their line of work.

Along the way through the pillars and gems of analysis we all should know, Simon gives us plenty of historical vignettes but wisely refrains from getting into the nomenclature wars of who should get proper credit for what. The past is the past. Renaming theorems just to set the record 
straight only serves to confuse the reader who probably already knows "X's Theorem" whether or not X was the first to prove it-or prove it at all. For example, Pythagoras probably did not prove the "Pythagorean theorem."

As someone who teaches at a liberal arts college, where we present mathematics as part of a balanced holistic undergraduate education, I certainly value these historical references since they show the development of analysis beyond just the names attached to the theorems. This series of books emphasizes that analysis developed over a long period of time with stumbles and false starts along the way. Simon also points out the historical figures, often with very brief, but well-resourced biographies, to show their influence in the development of analysis. Some, such as Euler and von Neumann, were great. Others, such as Blaschke, were questionable. Bieberbach was just plain rotten.

Simon's presentation sorts it all out and, as stated early on in his first volume, he aims for the best and most insightful proofs that he has gathered up throughout the years. He claims early on that he is not a historian. However, from the careful scholarship and the numerous references to original sources, he sure acts like one and the reader should certainly appreciate his doggedness to chase this all down for us. Indeed, there are 3,701 references in these five volumes, many of them dating back to the beginnings of analysis.

Simon's aim in this project is twofold. First, these books are to be used by instructors of graduate analysis classes such as real analysis, functional analysis, complex analysis, harmonic analysis, and operator theory. With the volume of material, an instructor needs to be selective about what they choose, but Simon gives sage guidance on what needs to be covered and what can be used as bonus material to suit an instructor's particular interests. Second, these books are to be used as references for the researcher who needs to re-heat a forgotten topic (Riemann-Stieltjes integration, self-adjoint extensions of operators) or perhaps fill in a gap in their analysis education (tensor products of Hilbert spaces, Brownian motion, gap series). As Simon is keenly aware of the volume of material he has produced, he helps the reader ferret out what is truly important with his "Big notions and theorems" section at the beginning of every chapter. His "Bonus sections" and "Bonus chapters" indicate that although the reader might not need this material at first, it would be very wise to learn it...eventually.

Since these books are for graduate courses in analysis, Simon assumes a certain amount of mathematical analysis maturity. Simon provides a review of some basics, not so much as to teach or even re-teach this material, but to fill in any gaps and to initiate the reader to both the level of difficulty and to the notation he will use throughout these volumes. Though the material is presented in a thoughtful and friendly way, there is a certain amount of seriousness and erudition to the presentation and the reader, both student and researcher, will definitely need to pay close attention to details and have pencil and paper at the ready.
Simon has a mission to present the best quality versions of theorems (e.g., the broadest version of the Cauchy integral formula)-often at the cost of some extra legwork on the part of the reader who needs to work through some advanced background material.

As part of Simon's dedication to being an analysis educator, there is a very generous helping of problems at the end of each section that serve several purposes. First, they point out the necessity of certain hypotheses of the theorems since often something goes terribly wrong when they are left out. Furthermore, these exercises force the student reader to work through some of the technical details of the proofs. Indeed, there is so much covered in this five-volume set that Simon can't chase down every last detail. Instead, he puts some of these details, or extensions of these details, as exercises with gentle nudges to help the student along. Still further, Simon uses the exercises to give the reader a chance to learn some interesting tidbits that expand what is covered in the chapter. For example, in the complex analysis part of the series (Part II A), there is a wonderful selection of power series problems that display various aspects of the issue of a "natural boundary" via the series of Lambert, Hadamard, and Weierstrass. Simon helps the reader through these examples by giving them bite-size chunks to work through and then assemble as a bona fide theorem at the end. As mentioned earlier, all of this extra material in the exercises is meticulously referenced, often back to its original sources. Of course, there are plenty of exercise (drill) type problems that give the student an opportunity to work through some standard gems.

This course, in full or in parts, was beta tested by a halfdozen or so institutions, and there was plenty of input on these volumes from more than thirty of some of the best names in analysis. The analysis community has spoken. This is the core of analysis. Learn it!

I feel obligated as part of this review to not just talk about Simon's series in general, as I have done thus far. But, if the reader is going to invest in this series, they should know what they are getting. Even though surveying some of the topics covered in these volumes might not be the best way to spend valuable space in a book review, here is some of what you are getting.

Part 1 (Real Analysis) begins with some preliminaries (set theory, topology, linear algebra) and continues with topological spaces, measure theory, Lebesgue theory, Hilbert spaces, convexity in Banach spaces, distributions, with "bonus" material on topics that include Haar measure, probability, fixed point theorems, Brownian motion, and Banach space valued functions.

Parts 2 and 2 A (Basic Complex Analysis, Advanced Complex Analysis). The first of these two volumes cover the Cauchy theory (Cauchy integral theorem and formula in various stages of generality) and its consequences (argument principle, Rouchés theorem, open mapping theorem, maximum modulus, singularities, harmonic functions) along with 
approximation theorems, Riemann mapping theorem, uniformization theorem, and the Paley-Weiner theory. The second covers more specialized, but still important, topics such as the Poincaré metric, applications to number theory, asymptotic methods, univalent functions, and the Nevanlinna theory.

Part 3 (Harmonic analysis) is the second largest of these volumes (Part 1 is the largest-but only by a bit). Here, after some calculus, complex analysis, and real analysis reminders ( $L^{p}$ spaces, Fourier analysis, probability, Green's theorem, Blaschke products), Simon proceeds to maximal functions, harmonic and subharmonic functions, classical Hardy space theory, with bonus chapters on wavelets, Calderón-Zygmund theory, Sobolev spaces, and Tauberian theorems.

Part 4 (Operator Theory) takes the reader from linear algebra to self-adjoint extensions of operators. As Simon points out in the introduction to this volume, there is some controversy here. His focus is on "mainly self-adjoint and/ or compact operators on Hilbert space" and hence he omits, for example, the beautiful model theory of Sz.-Nagy and Foias, and several other topics that concern non-normal operators. Simon's operator theory volume covers the basics of bounded operators on Hilbert/Banach spaces, including the development of compact operators. Included in this discussion is the Ringrose-West decomposition theorem for compact operators on a Hilbert space, a beautiful result not always covered in Hilbert space books. This volume also includes a chapter on orthogonal polynomials (Simon also has several imposing books on that topic) and a very thorough treatment of the spectral theorem, served three ways, for self-adjoint operators. Along the way, there is a discussion of perturbation theory for self-adjoint operators, where again, this important material is not always presented in well-known texts. Finally, as befitting a mathematical physicist, there is an excellent treatment of unbounded self-adjoint operators as well as operators that have self-adjoint extensions. The Schrödinger and Sturm-Liouville theory plays an important role here.

Though the title of these volumes is A Comprehensive Course in Analysis, Simon had to make some difficult choices of what to leave out. Some of your favorite topics, and maybe even your research area, might be missing from the main texts or perhaps relegated to the exercises-or maybe not appear at all. There are many reasons why topics do not appear. Maybe there are already some excellent and thorough texts of these subjects. Why try to re-write a perfectly good text when directing the reader to this text, via the references, will do? I couldn't resist and looked up several of my favorite operator theory topics to see if they "made the cut" and were mentioned in either the historical notes or the references. Some did while others did not. Maybe some of these excluded fields had their moment in the sun and Simon feels the need to focus elsewhere. Maybe some of these fields are struggling and need to re- prove their worth to be reconsidered for readmission to the corpus of analysis. Either way, Simon maintains a Facebook page for A Comprehensive Course in Analysis to keep us up to date with revisions or updates.

Finally, perhaps I have been somewhat breezy and informal in this review and my language was a bit too friendly. However, I think this reflects the type of language Simon uses in his series. There are plenty of contractions ("we'll") and exclamation points to express excitement for analysis ("...this was proved by de Branges about 70 years after the conjecture was made!"). There are even catchy titles of sections ("The magic of maximal functions," "A warmup: the Euler product formula"). Indeed, Simon even admits "I have relied at times-horrors!- on information from the Internet." There is a certain amount of honesty, humility, genuineness, and dare I say, playfulness, in Simon's writing that draws readers in and welcomes them into the wonderful world of analysis.

\section{Credits}

Author photo is courtesy of the author. 


\section{WHAT'S HAPPENING AT THE AMS BOOTH?}

$\% \quad 40 \%$ off the list price of AMS titles for AMS \& MAA members

$25 \%$ discount for all other JMM attendees

Free shipping on all book purchases

2 Complimentary access to MathSciNet ${ }^{\circledR}$ for the duration of the meeting: Visit mathscinet.ams.org while connected to the conference Wi-Fi network or from the Wi-Fi network at any of the conference hotels.

\section{EVENTS YOU WON'T WANT TO MISS:}

9:30 am-5:00 pm | Thank Your Mentor: Visit the Campaign for The Next Generation and thank someone who helped you get started in mathematics; then enter to win a mini printer!

12:15-5:30 pm | Mathematics Research Communities (MRC) Alumni, stop by the booth and pick up your ribbon for your MRC year!

2:15 pm MathSciNet Demonstration: Learn how MathSciNet can help you with your research.

4:00 pm | Meet Erica Flapan, the new Editor in Chief of the Notices of the AMS.

5:00 pm | Grand Opening Raffle winner announcement: Stop by and enter!

\section{9:30 am-5:00 pm | Thank Your Mentor}

9:30 am-4:25 pm | Professional Portrait Sessions for AMS Members: Have your professional portrait taken and emailed to you in just a few minutes. Schedule your appointment at amermathsoc.simplybook.me.

2:00-4:00 pm | What do you think about ams.org? Receive a free AMS gift when you stop by to tell us what you like or dislike about our website.

2:15 pm | MathSciNet Demonstration

\section{9:30 am-5:00 pm | Thank Your Mentor}

9:30 am-4:25 pm | Professional Portrait Sessions for AMS Members

2:00-4:00 pm | What do you think about ams.org?

2:15 pm | MathSciNet Demonstration

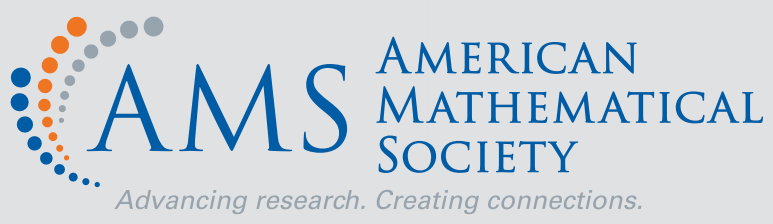




\section{BOOKSHELF}

\section{New and noteworthy titles on our bookshelf January 2019}

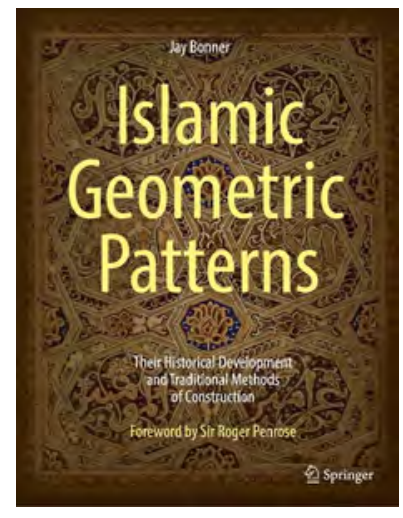

Islamic Geometric Patterns:

Their Historical Developments

and Traditional Methods of

Construction

by Jay Bonner, with a

Foreword by Roger Penrose

(Springer, 2017, 595 pages).

Islamic artwork is renowned for its intricate and awe-inspiring use of complex geometric patterns. As Roger Penrose states in the foreword, "there is a distinctive beauty in these designs, most of these patterns being of a particular geometrical character, demonstrating a keen and subtle knowledge and interest in geometry and a profound skill in using geometrical motifs to produce some incredibly intricate patterns."

This monumental work contains a staggering 540 figures, almost all of which are in color. Islamic Geometric Patterns could certainly function as a "mathematics coffee-table book" since one cannot help browsing through its pages to ponder the many colorful designs carefully described therein.

The book is divided into four major sections. The first chapter, which is approximately 150 pages long, concerns the historical development of the art form. It features many full-color photographs of the original artwork. In the second portion (about seventy pages), the author provides a geometric classification of the mind-boggling array of patterns featured throughout the Islamic world. The third and longest chapter (over three hundred pages) describes the geometrical techniques that underpin the art form. Everything here is explained in great detail, and one feels that motivated readers might even be able to (with great patience and practice) generate such patterns on their own. The final chapter, written by computer scientist Craig Kaplan, is the shortest (a little over twenty pages). It focuses on computer algorithms for the production of geometric patterns of the type described in the book.

The Bookshelf is prepared monthly by Notices Associate Editor Stephan Ramon Garcia.

Appearance of a book in the Notices BookShelf does not represent an endorsement by the Notices or by the AMS.

Suggestions for the BookShelf can be sent to notices-book1ist @ams.org.

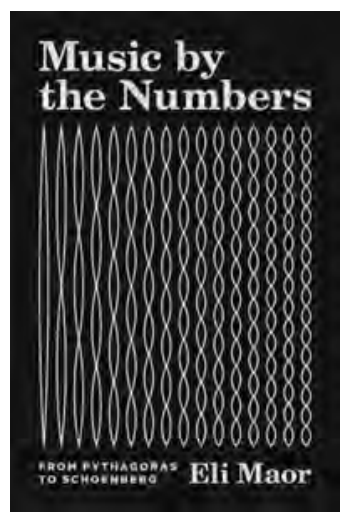

Music by the Numbers: from Pythagoras to Schoenberg by Eli Maor (Princeton, 2018, 176 pages).

This book concerns the long-running relationship between math and music. It should be appealing to any mathematically oriented reader with some background in music. Familiarity with musical terminology and notation would be a plus.

The first chapter sets the stage and provides a whirlwind tour of physics, mathematics, and western music at the dawn of the twentieth century. The second chapter reaches back to the Pythagoreans and the problem of the vibrating string. From this point the approach is largely chronological, except for five "Sidebars" that are between two and four pages each. These concern related topics, such as using a Slinky to provide a slow-motion model for the vibrating string.

The math involved is not deep and should be accessible to an attentive calculus student. On the other hand, the treatment of music requires a degree of familiarity. For example, the mathematical approaches to composition embraced by Bach and Schoenberg can be explained in words, but only the ear can truly understand the difference.

The reader should be aware that the music discussed belongs almost exclusively to the western tradition. More specifically, the book concerns not only old favorites such as Bach, Beethoven, and Mozart, but also more modern composers, such as Bernstein, Cage, and Schoenberg. Schoenberg's mathematical approach to atonal composition plays a central role in the later chapters.

The discussion touches on physics early and often. Sometimes the link is direct, such as the study of the vibrating string and the development of Fourier series. At other points, the connections are more abstract. For example, Einstein's theory of relativity is discussed in parallel with Schoenberg's development of twelve-tone music. Einstein's theory tells us that there is no universal, privileged frame of reference. As opposed to most Western music, Schoenberg's approach is more "relativistic," in the sense that all twelve tones are utilized with somewhat equal importance. 


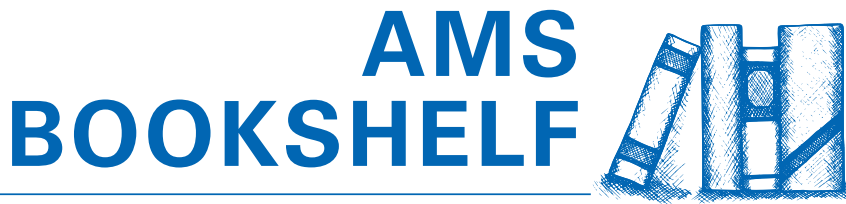

The AMS Book Program serves the mathematical community by publishing books that further mathematical research, awareness, education, and the profession while generating resources that support other Society programs and activities. As a professional society of mathematicians and one of the world's leading publishers of mathematical literature, we publish books that meet the highest standards for their content and production. Visit bookstore.ams.org to explore the entire collection of AMS titles.

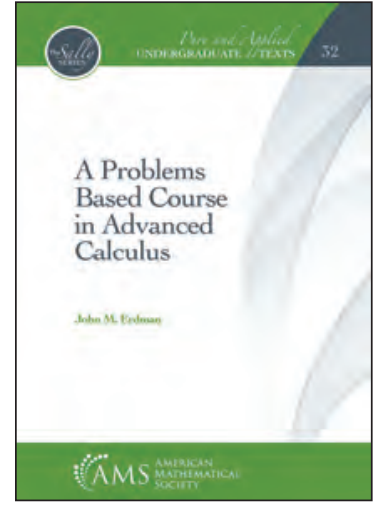

A Problems Based Course in Advanced Calculus (AMSTEXT/32, 2018) By John M. Erdman

As math courses in many colleges are transitioning to less lecturing and more interactive problem-driven formats, many instructors have gone away from traditional textbooks and have developed and honed their own course notes as they adapt their teaching methods to the needs of their students.

John Erdman's A Problems Based Course in Advanced Calculus is a successful example of the kind of book that can emerge from such a program. The book provides not only a useful list of problems, but also a compelling overall narrative, helpful words of guidance, and of course an emphasis on exercises.

The tone of the book reflects the author's years of experience balancing the need to give students helpful guidance while maintaining the principle that less teaching leads to more learning. All this culminates in prose that is conversational and inviting yet efficient and economical, allowing plenty of room for the reader to discover for themselves.

This book is aimed at students who have taken calculus and linear algebra but have little or no background in proof-writing. Using an appendix that introduces proof-writing basics, and three well-crafted narrative arcs, the author leads students from one-variable calculus with proofs, including notions like open and closed intervals and topological definitions of continuity and limits, to increasingly abstract notions including metric topology and normed linear spaces; and finally applies these ideas to multi-variable differential calculus.

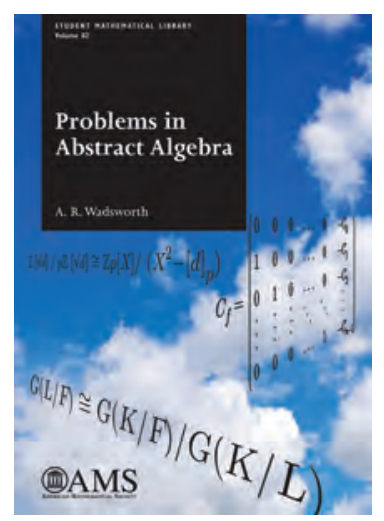

Problems in Abstract Algebra (STML/82, 2018) by A. R. Wadsworth

I have heard multiple calls, from students and instructors, for a massive database of exercises available on the web. But, as with collections of poetry, or paintings, there are clear advantages to a thoughtfully curated list. Wadsworth's book has the individual mark of a selection informed by the author's experience both as a mathematician who regularly applies the ideas and their offshoots in his research, and as a longtime teacher of the subject.

Problems in Abstract Algebra is self-contained, and runs through the usual topics of undergraduate (and beginning graduate) abstract algebra: group and rings, linear algebra, and Galois Theory. The main difference between this book and a standard textbook is that instead of complete proofs of standard results, the author only briefly sketches the ideas, and moves quickly from set up to exercises. In this way, students can actively experience for themselves the process of bringing the ideas to light.

For students, working through the exercises in this book is a great way to supplement an abstract algebra course. The book would also be ideal for a student preparing for graduate school, and wanting to review and test their knowledge. From the point of view of a teacher, the organization of topics provides a useful guide to pacing a course; and the selection of reliably good examples and exercises is a resource, not only for composing homework and examinations, but also for providing ideas for extra topics and commentary.

The AMS Bookshelf is prepared monthly by AMS Senior Editor Eriko Hironaka. Heremail address is exh@ams.org. 


\section{Fifty Years of CBMS
Regional Conferences}

\section{David M. Bressoud}

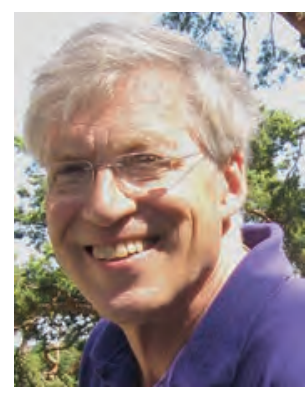

David M. Bressoud
The year 2019 marks the 50th anniversary of the CBMS/ NSF Regional Conference Series ${ }^{1}$ and the accompanying CBMS monographs https: //bit . 1y/2nZHqdN, one of the hallmark efforts of the Conference Board of the Mathematical Sciences. Since its inception in 1969, 372 of these conferences have been run, leading to the publication of more than 230 monographs. This is an opportune moment to look back on the success of this program and to look forward. While the essence of these conferences will not change, CBMS and NSF have discussed modifications that will be in place by 2020 .

\section{Origins}

CBMS was incorporated in 1960 as a forum for bringing together the presidents of the professional societies in the mathematical sciences to coordinate their efforts. Toward the end of that first decade it formulated two projects that spanned the interests of multiple societies: the departmental survey, which has been conducted every five years, and the Regional Conference Series, originally run under the auspices of both AMS and the Society for Industrial and Applied Mathematics (SIAM).

To distinguish them from the many other mathematical conferences run each year, the Regional Conferences were conceived with three distinctive features:

1. They would be regional, spread across the United States and favoring institutions that would normally not host major research conferences. The intent was to facilitate

David Bressoud is DeWitt Wallace Professor of Mathematics at Macalester College and Director of the Conference Board of the Mathematical Sciences. His email address is Bressoud@macalester. edu.

${ }^{1}$ https://www.cbmsweb.org/regional-conferences/. The work of CBMS on the Regional Conference Series is supported by NSF grants \#1709265 and $\# 1804259$. The opinions expressed in this article do not necessarily reflect those of the National Science Foundation.

For permission to reprint this article, please contact: reprint -permission@ams .org.

DOI: http://dx.doi.org/10.1090/noti1754 access to at least one of these conferences for all mathematical scientists and to promote the formation of local research groups.

2. They would feature one leading researcher who would give a series of ten lectures over five days, leading the participants from a basic introduction in an important area of current research to the point where they could begin to work in this field.

3. The conference would be followed by the production of a monograph, based on the ten lectures, that would serve the broader population of mathematical scientists as an introduction to an exciting realm of contemporary mathematical research.

The first two monographs, both products of 1969 conferences, were Irving Kaplansky's Algebraic and Analytic Aspects of Operator Algebras [8], published by AMS, and Garett Birkhoff's The Numerical Solution of Elliptic Equations [1], published by SIAM. In the early years, SIAM published the monographs in statistics, but starting with Morris Eaton's Group Invariance Applications in Statistics [3], based on a 1987 conference, the American Statistical Association and the Institute of Mathematical Statistics have jointly published the monographs in statistics. There are now three monograph series: the CBMS Regional Conference Series in Mathematics (AMS), the CBMS-NSF Regional Conference Series in Applied Mathematics (SIAM), and the CBMS-NSF Regional Conference Series in Probability and Statistics (IMS \& ASA).

\section{Current Status}

The program has been an enormous success. Total sales of the monographs have far exceeded a quarter of a million copies. Five have gone into second editions $[5,6,10,11,14]$, and three have sold over 10,000 copies each $[2,4,13]$. One of these, Ingrid Daubechies' Ten Lectures on Wavelets, based on her 1990 CBMS Conference at the University of Lowell, has sold over 16,000 copies, been cited almost 30,000 
times, and, in 1994, received the AMS Steele Prize for Excellence in Expository Writing.

CBMS has always managed the infrastructure for these seminars: encouraging applications to NSF to run one of these conferences and disseminating information on the application process, publicizing the conferences, handling the honoraria, encouraging speakers to turn in the monographs, and approving the monographs for publication.

The CBMS lecturers have included four Fields Medalists. Paul Cohen at Carleton College in 1972 and William Thurston at the University of Minnesota in 1983 gave their lectures after receiving the medal. Vaughan Jones at the U.S. Naval Academy in 1988 and Terrence Tao at New Mexico State University in 2005 lectured before they received the medal. Women have been underrepresented as conference lecturers, but the past five years have included Wen-Ching Winnie Li [9], Nalini Joshi, Alice Guionnet, and Anna C. Gilbert among the CBMS lecturers.

Geographic, institutional, and subject diversity continue to be distinguishing features of the conference series. In the past five years regional conferences have been held in Alabama, California, Illinois, Iowa, Maryland, Minnesota, Montana, New Hampshire, New Mexico, New York, North Carolina, North Dakota, Ohio, Oklahoma, South Carolina, Texas, Utah, Washington DC, and Wyoming. They have been run at Historically Black Colleges and Universities and Hispanic Serving Institutions, at liberal arts colleges, at regional comprehensive universities, and at research universities. Topics have ranged from combinatorics, number theory, geometry/topology, algebra, and analysis to PDEs, data analysis, statistical analysis, computational methods, and quantum field theory, often focusing on exciting new developments where these subjects overlap.

In the early days, CBMS and its advisory board received a block grant from NSF, with total control over which con-

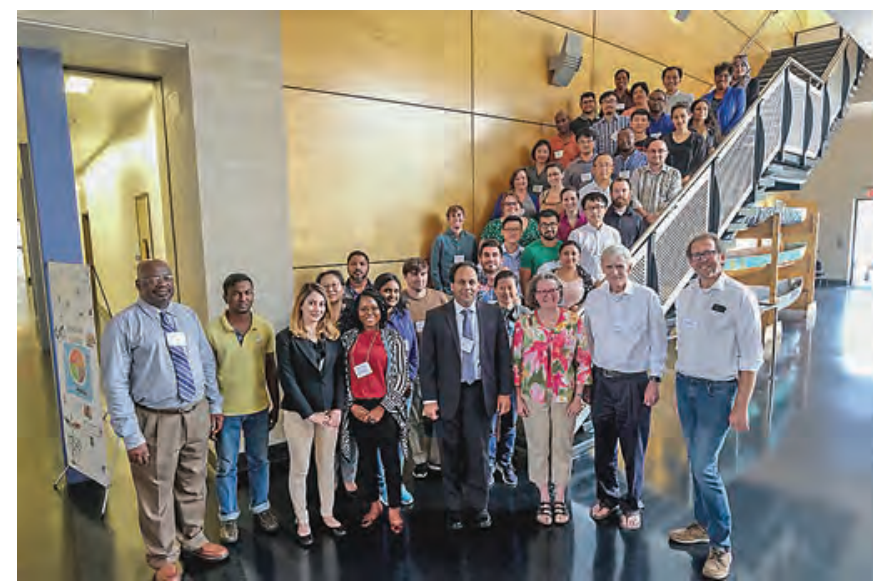

Participants at the CBMS-NSF Conference on Computational Methods in Optimal Control by William Hager at Jackson State University, July 23-27, 2018, funded by NSF grant \#1743826. ferences would be supported and at what level of funding. Over the decades, the funding model has changed. Applications to organize a CBMS conference are now made directly to NSF, usually with an April deadline to run a conference in the summer of the following year. Until recently, CBMS played a role in deciding which conferences would be funded. Moving forward, CBMS will play a more active role in soliciting applications and proposal development, and will not participate in funding decisions.

\section{Evaluating the regional conference series}

Early in 2017, in response to concerns that the number of proposals to run one of these conferences had been in decline in recent years, that monographs were coming from an ever smaller fraction of conference speakers, and that the citation numbers for recently produced monographs were also down, CBMS undertook a thorough re-evaluation of the program.

In spring and summer 2017 the board held focus groups and sent questionnaires to many of those who had been involved with CBMS conferences over the past fifteen years. The issues targeted for discussion included whether CBMS should continue to build the conferences around a single speaker giving ten lectures, whether to continue to produce monographs, and how to take advantage of the opportunities afforded by the internet. While responses varied greatly, there were three clear messages that came through:

1. A single lecturer is neither necessary nor always desirable. However, there was consistent support for the rationale behind the requirement of a single speaker: to provide a well-connected and tightly focused flow that moves from a basic introduction to the topic up to the edge of current research, giving participants an intensive experience that effectively introduces them to the important tools and problems. Many mathematicians felt that this could be accomplished more effectively with more than one voice, provided there was tight coordination in linking the talks.

2. There was almost universal support for continuation of the monographs. This came from senior mathematicians who treasure their dog-eared copies of past monographs, but also from current graduate students who continue to find them to be a useful guide into a field they wish to study. It was gratifying to see that print still has an important role to play in disseminating mathematical knowledge.

3. There also was strong support for insisting that all conferences produce a body of timely online material, from background material that should be posted before the conference begins to videos of the conference talks and detailed notes that expand on the actual talks.

We also heard of the importance of the networking that happens at these conferences and the need for opportunities to work collectively on problems and to gain experience with the software that is often an integral part of particular research streams. 
Ultimately, it was clear that CBMS, through its regional conferences, is uniquely situated to facilitate the placement of research conferences at institutions and in locations where they would not normally be found, thus helping to build the community of those actively engaged in mathematical research.

\section{Changes to the regional conference series}

Going forward, in response to community and stakeholder feedback, CBMS has implemented some changes in the conference series:

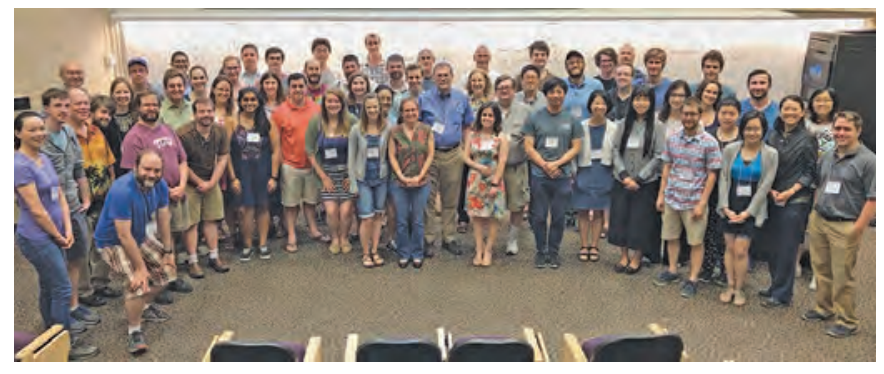

Participants at the CBMS-NSF Conference on Applications of Polynomial Systems by David Cox at Texas Christian University, June 4-8, 2018, funded by NSF grant \#1741730.

- CBMS will take a more active role in identifying both potential lecturers and potential conference sites. To assist in this effort, an Advisory Board has been constituted whose initial members are Susmita Datta, University of Florida; David Donoho, Stanford University; Bengt Fornberg, University of Colorado, Boulder; Ken Ono, Emory University; and Mary Lou Zeeman, Bowdoin College. In addition, CBMS will offer assistance in crafting strong proposals.

- The format of ten lectures over five days will be maintained, but these could be given by as many as three people provided there is only one "principal lecturer" who plans and coordinates the talks so that they stay tightly focused and build from a basic introduction to questions of current research.

- In addition, while lectures provide an efficient means of communicating a lot of information in a short space of time, by themselves they are not sufficient to engender the kind of research activity this series intends to promote. Participants, especially those new to the field, must be nurtured through interaction with others at the conference. Facilitating this interaction is encouraged, whether through problem sessions, laboratory experiences, small group Q\&A and discussions, or other means.

- There will be new expectations for materials to be posted on the CBMS website:
- The principal lecturer will provide preliminary materials that will include an introduction to the problems to be discussed. This will supply some historical context, a reading list, basic definitions and results relevant to the upcoming conference, as well as an outline of the ten talks to be given.

- The principal lectures will use prepared slides (e.g. PDF, PPT, or Beamer), which will be submitted to CBMS by the end of the conference.

- The conference organizers will video record at least the ten principal lectures, which CBMS will offer on Vimeo or a comparable site.

- The principal lecturer will post a fleshed out version of the outline of the ten talks within 45 days of the conclusion of the conference.

- A polished monograph is still expected. It is hoped that the extended outline of the talks will be a significant step toward its production.

- The hallmark emphasis on institutional and geographic diversity will be expanded to include diversity of lecturers and participants, with an emphasis on broadening participation by members of groups underrepresented in the mathematical sciences.

When, in 2017, CBMS contacted those who had recently been involved with this series, we were heartened by the outpouring of support that we received. While not everyone agreed on the way forward, everyone communicated the value of this series, both in the past and for the future. We are grateful for the legacy of the past fifty years and for this opportunity to re-imagine this series as we move into the next decades.

\section{References}

[1] Birkhoff G. The numerical solution of elliptic equations, CBMS-NSF Regional Conference Series in Applied Mathematics, 1, Published for the Conference Board of the Mathematical Sciences, Washington, DC; by the Society for Industrial and Applied Mathematics, Philadelphia, PA, 1972, MR0311125

[2] Daubechies I. Ten lectures on wavelets, CBMS-NSF Regional Conference Series in Applied Mathematics, 61, Published for the Conference Board of the Mathematical Sciences, Washington, DC; by the Society for Industrial and Applied Mathematics, Philadelphia, PA, 1992, MR1162107

[3] Eaton ML. Group Invariance Applications in Statistics, CBMS-NSF Regional Conference Series in Probability and Statistics, 1, Published for the Conference Board of the Mathematical Sciences, Washington, DC; by the Institute of Mathematical Statistics, Hayward, CA and the American Statistical Association, Alexandria, VA, 1989

[4] Efron B. The jackknife, the bootstrap, and other resampling plans, CBMS-NSF Regional Conference Series in Applied Mathematics, 38, Published for the Conference Board of the Mathematical Sciences, Washington, DC; by the Society for Industrial and Applied Mathematics, Philadelphia, PA, 1982, MR0659849

[5] Graham R, Butler S. Rudiments of Ramsey theory: Second edition, CBMS Regional Conference Series in Mathematics, 
123, Published for the Conference Board of the Mathematical Sciences, Washington, DC; by the American Mathematical Society, Providence, RI, 2015. MR340921

[6] Huber PJ. Robust Statistical Procedures: Second edition, CBMS-NSF Regional Conference Series in Applied Mathematics, 68, Published for the Conference Board of the Mathematical Sciences, Washington, DC; by the Society for Industrial and Applied Mathematics, Philadelphia, PA, 1996, MR1420192

[7] Jones VFR. Subfactors and Knots, CBMS Regional Conference Series in Mathematics, 80, Published for the Conference Board of the Mathematical Sciences, Washington, DC; by the American Mathematical Society, Providence, RI, 1991. MR1134131

[8] Kaplansky I. Algebraic and Analytic Aspects of Operator Algebras, CBMS Regional Conference Series in Mathematics, 1, Published for the Conference Board of the Mathematical Sciences, Washington, DC; by the American Mathematical Society, Providence, RI, 1970. MR0312283

[9] Li W. Zeta and L-functions in number theory and combinatorics, CBMS Regional Conference Series in Mathematics, Published for the Conference Board of the Mathematical Sciences, Saint Paul, MN; by the American Mathematical Society, Providence, RI, In Press.

[10] Rheinboldt WC. Methods for solving systems of nonlinear equations: Second edition, CBMS-NSF Regional Conference Series in Applied Mathematics, 70, Published for the Conference Board of the Mathematical Sciences, Washington, DC; by the Society for Industrial and Applied Mathematics, Philadelphia, PA, 1998. MR1645489

[11] Spencer J. Ten lectures on the probabilistic method: Second edition, CBMS-NSF Regional Conference Series in Applied Mathematics, 64, Published for the Conference Board of the Mathematical Sciences, Washington, DC; by the Society for Industrial and Applied Mathematics, Philadelphia, PA, 1993, MR1249485

[12] Tao T. Nonlinear dispersive equations: Local and global analysis, CBMS Regional Conference Series in Mathematics, 106, Published for the Conference Board of the Mathematical Sciences, Washington, DC; by the American Mathematical Society, Providence, RI, 2006. MR2233925

[13] Tarjan RE. Data structures and network algorithms, CBMSNSF Regional Conference Series in Applied Mathematics, 44, Published for the Conference Board of the Mathematical Sciences, Washington, DC; by the Society for Industrial and Applied Mathematics, Philadelphia, PA, 1983, MR0826534

[14] Temam R. Navier-Stokes equations and nonlinear functional analysis: Second edition, CBMS-NSF Regional Conference Series in Applied Mathematics, 66, Published for the Conference Board of the Mathematical Sciences, Washington, DC; by the Society for Industrial and Applied Mathematics, Philadelphia, PA, 1995, MR1318914

\section{Credits}

Author photo is courtesy of Jan Bressoud.

Photo of participants at the Cox conference is courtesy of Derek Isensee.

Photo of participants at the Hager conference is courtesy of Tor A. Kwenbe.

\section{Recent volumes from MSJ}

Advanced Studies in Pure Mathematics http://mathsoc.jp/publication/ASPM/

Volume 78

Singularities in Generic Geometry

Edited by S. Izumiya (Hokkaido),

G. Ishikawa (Hokkaido),

M. Yamamoto (Hirosaki), K. Saji (Kobe),

T. Yamamoto (Tokyo Gakugei),

M. Takahashi (Muroran Inst Tech)

ISBN 978-4-86497-055-6

Volume 77

The 5oth Anniversary of Gröbner Bases Edited by $\mathrm{T}$. Hibi (Osaka)

ISBN 978-4-86497-052-5

Volume 76

Representation Theory, Special Functions and Painlevé Equations

RIMS 2015

Edited by H. Konno (Tokyo Marine Sci. \& Tech.),

H. Sakai (Tokyo), J. Shiraishi (Tokyo), T. Suzuki (Kindai),

Y. Yamada (Kobe)

ISBN 978-4-86497-050-1

MSJ Memoirs

http://mathsoc.jp/publication/memoir/memoirs-e.html

Volume 36

Global solutions and

the asymptotic

behavior for nonlinear

wave equations with

small initial data

Soichiro Katayama

ISBN 978-4-86497-054-9

Volume 35

Foundations of

the minimal model program

Osamu Fujino

ISBN 978-4-86497-045-7

$\nabla \nabla \nabla$ For purchase, visit $\nabla \nabla \nabla$

http://www.ams.org/bookstore/aspmseries

http://www.worldscientific.com/series/aspm

http://www.worldscibooks.com/series/msjm

The Mathematical Society of Japan 34-8, Taito 1-chome, Taito-ku

Tokyo, JAPAN

http://mathsoc.jp/en/ 


\section{JMM 2019 LECTURE SAMPLER}

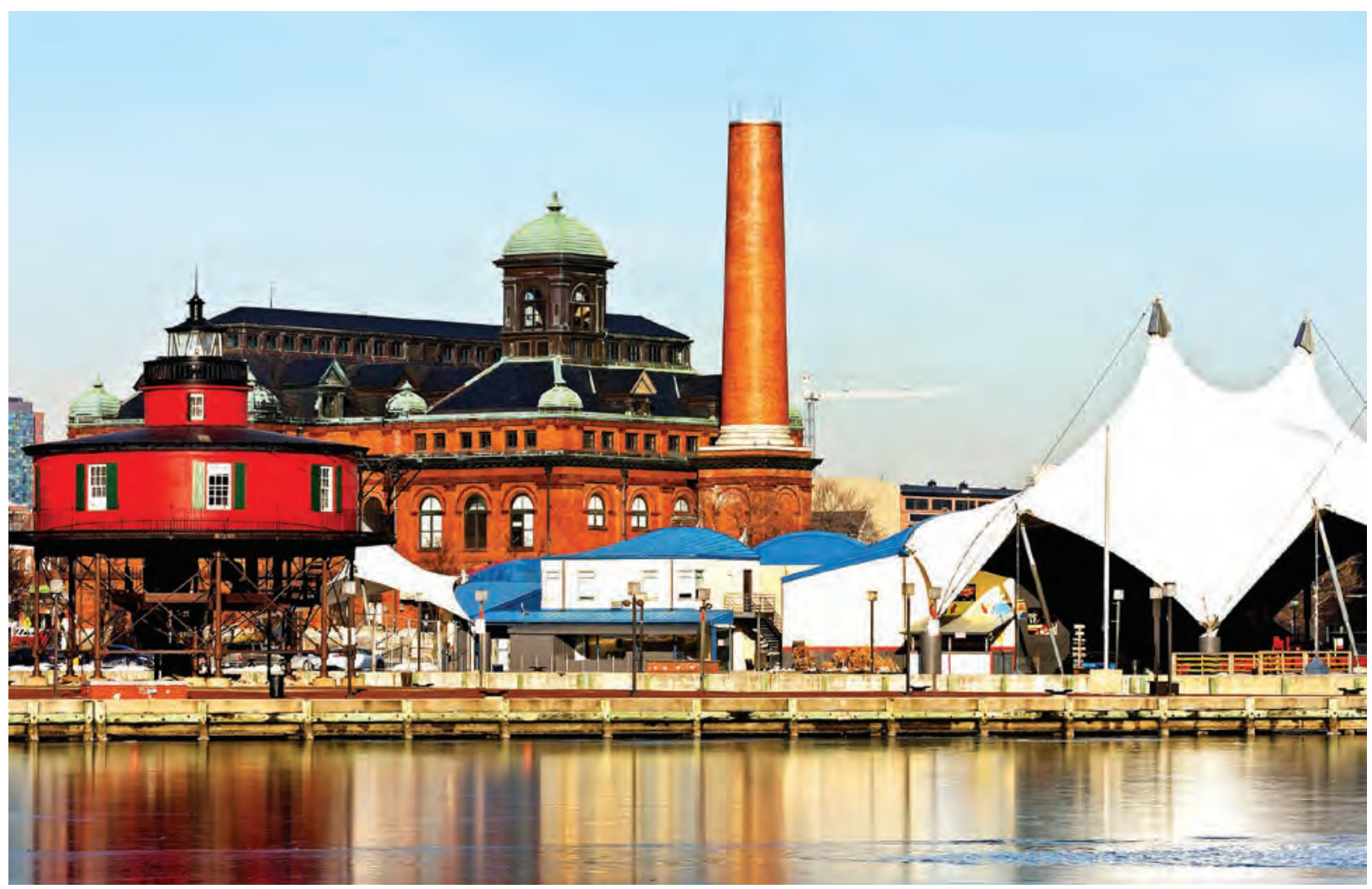

Baltimore Harbor.

Page 85 Jesús A. De Loera, "Algebraic, Geometric, and Topological Methods in Optimization" (AMS Invited Address)

10:05 am-10:55 am, Wednesday, January 16

Page 87 Amanda Folsom, "Symmetry, Almost"

(MAA Invited Address)

2:15 pm-3:05 pm, Wednesday, January 16

Page 89 Emmanuel Candes, "Sailing through Data: Discoveries and Mirages"

(MAA Invited Address)

3:20 pm-4:10 pm, Wednesday, January 16

Page 90 Edray Herber Goins, "A Dream Deferred:

50 Years of Blacks in Mathematics"

(MAA Invited Address)

9:00 am-9:50 am, Thursday, January 17

Page 92 Bryna Kra, "Dynamics of Systems with Low Complexity"

(AWM-AMS Noether Lecture)

10:05 am-10:55 am, Thursday, January 17
Page 94 Pamela E. Harris, "A Mathematical Journey of Culture, Community, and Collaboration" (MAA Invited Address) 9:00 am-9:50 am, Friday, January 18

Page 95 Karen Hunger Parshall, "The Roaring Twenties in American Mathematics" 10:05 am-10:55 am, Friday, January 18

Page 96 Talithia Williams, "A Seat at the Table: Equity and Social Justice in Mathematics Education" (NAM Cox-Talbot Address) 7:45 pm-8:35 pm, Friday, January 18

Page 97 Lillian B. Pierce, "On Torsion Subgroups in Class Groups of Number Fields" (AMS Invited Address) 9:00 am-9:50 am, Saturday, January 19

Page 99 Henok Mawi, "On Mathematical Problems in Geometric Optics" (NAM Claytor-Woodard Lecture) 1:00 pm-1:50 pm, Saturday, January 19

For permission to reprint this article, please contact: reprint - permission@ams.org.

DOI: http://dx.doi.org/10.1090/noti1783 


\section{JMM 2019 LECTURE SAMPLER}

\section{Algebraic, Geometric, and Topological Methods in Optimization}

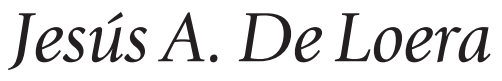

The great mathematician Lobachevsky is attributed with saying "there is no branch of mathematics, however abstract, which may not some day be applied to phenomena of the real world." There are plenty of examples of how this is evidently true for algebra (broadly including number theory too) and geometry, and topology. In my talk, accessible to students and non-experts, I will stress this point further by showing how algebraic and geometric thinking strongly influences the vibrant field of optimization. Algebraic, geometric, and topological methods permeate every subfield of optimization and have a history (see e.g., [2-4]), but let me pique your curiosity with one recent exciting example:

The linear optimization problem, often called the linear programming problem, seeks to maximize a linear functional under linear inequality and equation constraints. In matrix form we can write it as

$$
\text { Maximize } \mathbf{c}^{T} \mathbf{x} \text { subject to } A \mathbf{x}=\mathbf{b} \text { and } \mathbf{x} \geq 0 \text {. }
$$

Here $A$ is a real $m \times n$ matrix, $\mathbf{c}, \mathbf{b}$ are $n$ and $m$ vectors respectively. For a short introduction to linear optimization, see [6]. Linear optimization is a workhorse of optimization.
Without reliable fast algorithms for linear optimization, other more sophisticated optimization challenges would simply not be possible, for instance, linear optimization is essential to the branch-and-bound methods necessary to solve non-linear mixed-integer optimization problems. Linear programming is crucial in several external scholarly domains, e.g., data science, combinatorics and graph theory, classical geometry, and others. Here is one way in which algebraic geometry has helped us understand linear optimization.

Interior point methods have had a profound impact in modern optimization, and in applications in engineering and science. They are among the most computationally successful algorithms for linear optimization. In practical computations, interior point methods follow a piecewise-linear approximation to the central path, using Newton methods steps (see e.g., [7]). But in reality, the central path is the quintessential algebraic-geometric object; the central path is the algebraic curve given exactly by the following system of quadratic and linear polynomial equations

$$
A \mathbf{x}=\mathbf{b}, A^{T} \mathbf{y}-\mathbf{s}=\mathbf{c} \text { and } x_{i} s_{i}=\lambda \text { for } i=1,2, \ldots, n .
$$

Until recently optimizers had mostly looked at the central path as a discretized numeric approximation, but understanding the exact central path is important (after all, we are following it closely). For example, how "curvy" can the central path really be? The intuition is that curves with small curvature are easier to approximate with fewer line segments, with fewer Newton steps. Recently, using exciting new methods from tropical algebraic geometry [5], X. Allamigeon, P. Benchimol, S. Gaubert, and M. Joswig showed that the total curvature of the central path can grow expo-
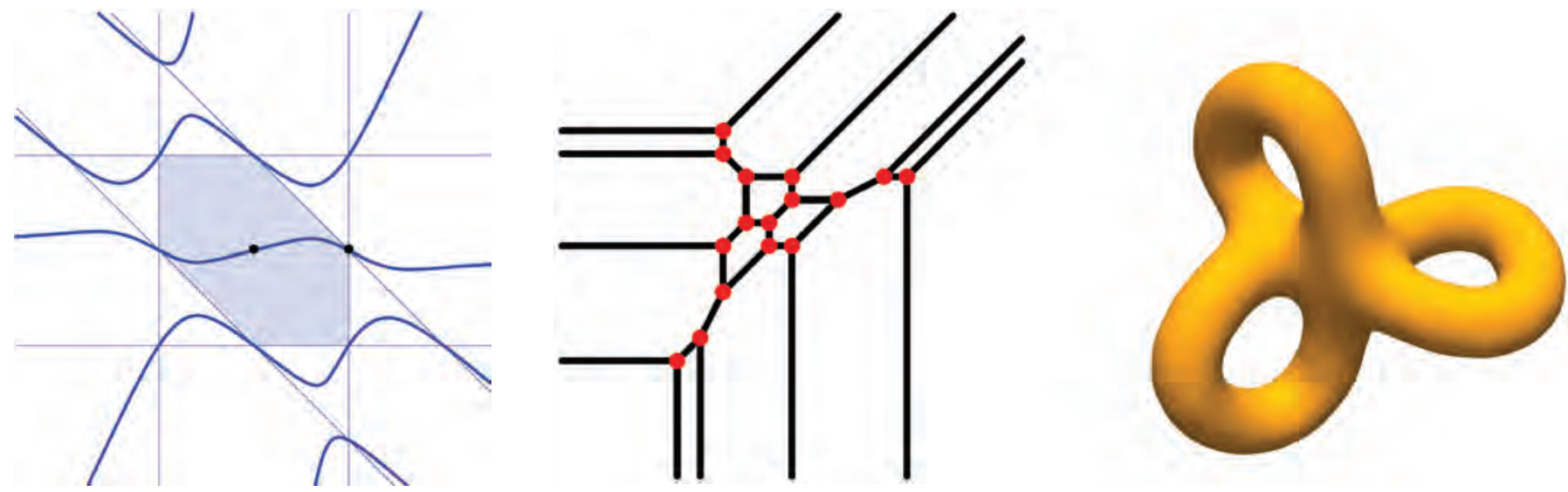

Figure 1. On the left a view of the entire central curve of a linear program projected to primal variable space. On the middle and right, a cartoon of the tropicalization of a genus three Riemann surface.

Jesús A. De Loera is a professor of mathematics at the University of California, Davis. His email is deloera@math. ucdavis. edu. He was partially supported by NSF grants DMS-1522158 and DMS-1818969.

For permission to reprint this article, please contact: reprint -permission@ams.org.

DOI: http://dx.doi.org/10.1090/noti1776 
nentially on the input data (see [1]). This implies that some interior-point methods cannot be strongly polynomial!

These techniques are fresh and unexpected, but there are many other examples like this with novel applications of algebraic and geometric techniques in optimization. I hope I tempted the reader to come and hear about opportunities for algebraists, topologists, and geometers to contribute to computational optimization. We will also organize a session on this topic. Join us in Baltimore! I promise my talk should be accessible to the non-expert and all students!

\section{References}

1. X Allamigeon, P Benchimol, S Gaubert, M Joswig. Log-Barrier Interior Point Methods Are Not Strongly Polynomial, SIAM Journal on Applied Algebra and Geometry 2(1): 140178, 2018. DOI 10.1137/17M1142132. URL https:// doi .org/10.1137/17M1142132

2. G Blekherman, P Parrilo, R Thomas, Semidefinite Optimization and Convex Algebraic Geometry, Society for Industrial and Applied Mathematics, 2012. MR3075433

3. J De Loera, R Hemmecke, M Köppe. Algebraic and Geometric Ideas in the Theory of Discrete Optimization, Society for Industrial and Applied Mathematics, 2012. MR3024570

4. M Grötschel, L Lovász, A Schrijver. Geometric Algorithms and Combinatorial Optimization, $2^{\text {nd }}$ ed. Algorithms and Combinatorics. Springer-Verlag, 1993. MR1261419

5. D Maclagan, B Sturmfels. Introduction to Tropical Geometry, Graduate Studies in Mathematics, vol.161. American Mathematical Society, 2015. MR3287221

6. J Matoušek, B Gärtner. Understanding and Using Linear Programming, Springer-Verlag, 2007.

7. J Nocedal, S Wright. Numerical Optimization, $2^{\text {nd }}$ ed. Springer Series in Operations Research and Financial Engineering. Springer, 2006. MR2244940

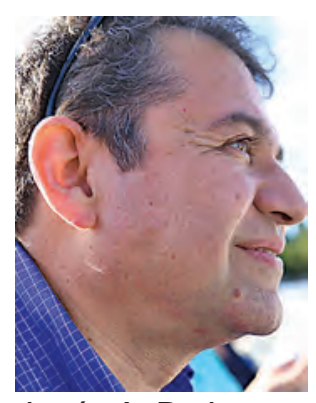

Jesús A. De Loera

\section{Credits}

Figure 1, left is courtesy of C. Vinzant; Figure 1, center and right are courtesy of $\mathrm{M}$. Joswig.

Author photo is by Antonio de Loera Brust. 


\section{Symmetry, Almost}

\section{Amanda Folsom}

Some definitions of the word symmetry include "correct or pleasing proportion of the parts of a thing," "balanced proportions," and "the property of remaining invariant under certain changes, as of orientation in space." One might think of snowflakes, butterflies, and our own faces as naturally symmetric objects-or at least close to it.
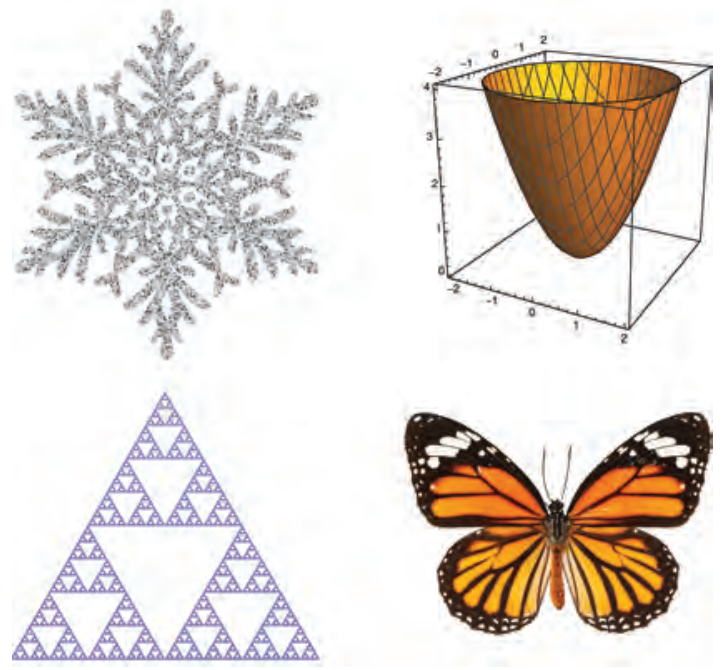

Figure 1. Natural and mathematical symmetries

Mathematically one can also conjure up many symmetric objects: even and odd functions, fractals, certain matrices, and modular forms, a type of symmetric complex function.

In more detail, modular forms, defined on the upper half of the complex plane $\mathbb{H}$, are (among other things) essentially symmetric with respect to the action of $\mathrm{SL}_{2}(\mathbb{Z})$ (the set of $2 \times 2$ integer matrices with determinant 1 ) on $\mathbb{M}$. A matrix $\left(\begin{array}{ll}a & b \\ c & d\end{array}\right)$ in the group $\mathrm{SL}_{2}(\mathbb{Z})$ acts on $\tau$ in $\mathbb{U}$ by fractional linear (a.k.a. Möbius) transformation, $\gamma \cdot \tau=$ $(a \tau+b) /(c \tau+d)$, and this action yields beautiful symmetries in $\mathbb{W}$.

Namely, in Figure 2, we see the upper half-plane divided into fundamental domains-loosely speaking, each region (including the gray shaded region, a standard fundamental domain) is a set of representatives for the orbits of the group action described above (where we must more precisely consider which boundary points to include). Due

Amanda Folsom is associate professor of mathematics at Amherst College. Her email address is afolsom@amherst.edu.

For permission to reprint this article, please contact:

reprint-permission@ams .org.

DOI: https://doi.org/10.1090/noti/1775

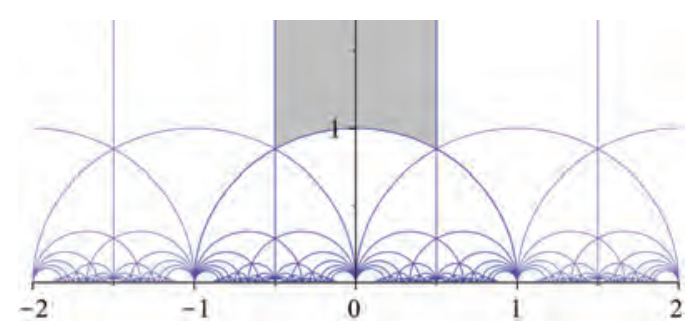

Figure 2. Fundamental domains

to the symmetries satisfied by a modular form, we need only to understand it on a fundamental domain in order to understand it everywhere in $\mathbb{H}$. For example, if $f$ is a modular function (a weight 0 modular form), it satisfies $f(\gamma \cdot \tau)=f(\tau)$ for all $\gamma \in \mathrm{SL}_{2}(\mathbb{Z})$. "Modular forms are everywhere," as the title of Don Zagier's 2017 birthday conference boasted, and their properties surpass aesthetics; e.g., modular forms are known to be connected to a number of well-known problems, including Fermat's Last Theorem, Monstrous Moonshine, the Birch and SwinnertonDyer Conjecture, and more.

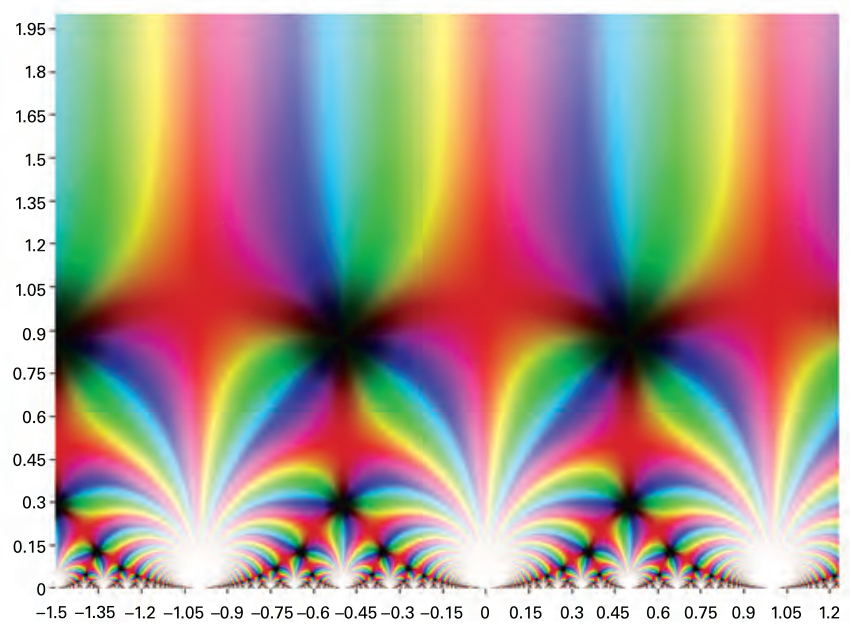

Figure 3. The modular $j$-function

In Figure 3, we see one interpretation of a graph of the modular function denoted by $j$, a hauptmodul which generates the field of modular functions. There, we see the upper half-plane, with the argument of a complex value of $j$ encoded by the color shown; red corresponds to the positive real axis, and movement in the counterclockwise direction runs through the colors of the rainbow. The magnitude of a complex value is represented by the darkness of the color. (For example, the figure suggests $j(0)$ is infinite, $j(i)$ (at center) is fairly large and real, and $j\left(e^{2 \pi i / 3}\right)$ is zero, all of which are true.) The symmetries of $j$ with respect to the tiling of $\mathbb{U}$ by fundamental domains suggested in Figure 3 are apparent.

All of the things discussed above, whether naturally symmetric or mathematically symmetric, exhibit a kind of beauty, so would they lose some of their innate beauty if their symmetries were altered? Alternatively, what could possibly be 


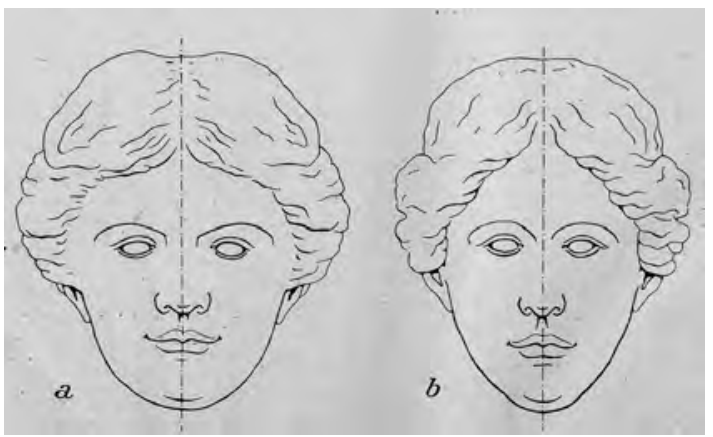

Figure 4. Facial symmetries

gained with slight symmetric imperfections?

Consider Figure 4, in which the images shown are rendered by reflecting one half of a drawing of a face over a central axis: what to make of any "biological preference" towards symmetric human faces? We now also have various notions of perturbed modular forms: as their names may suggest, quasimodular forms, false or partial theta functions, and mock modular forms are all not-quite-but-close to being modular in the sense of the symmetric characterization given above. For example, (with notation as above) a mock modular form $m$ satisfies $m(\gamma \cdot \tau)=\rho_{\gamma, \tau} m(\tau)+$ $h_{m}(\tau)$, for some (nontrivially produced) "error function" $h_{m}$ and explicit factor $\rho_{\gamma, \tau}$. Even more, we can get rid of the error to modularity $h_{m}$ attached to a mock modular form $m$. That is, by (nontrivially) adding to $m$ a suitable function $m^{-}$, the sum $\widehat{m}:=m+m^{-}$becomes more or less as symmetric as $j$ : we have $\widehat{m}(\gamma \cdot \tau)=\rho_{\gamma, \tau} \widehat{m}(\tau)$. But at what expense does $m$ gain symmetry by this addition of $m^{-}$?

If modular forms are everywhere, then perhaps mock modular forms are almost everywhere. Over the course of the last 10-15 years, a more general theory of harmonic Maass forms has developed, however, earlier footprints appear in Maass' work from the 1950s and, as we have more recently discovered, in Ramanujan's mock theta functions from 1920. The theory of mock modular forms has also seen applications in a number of subjects including combinatorics, mathematical physics, elliptic curves, quantum modular forms, and more.

This last subject, quantum modular forms, initially developed by Zagier in 2010, has been of particular interest lately. Like mock modular forms, quantum modular forms exhibit symmetry properties up to an error function as explained in the preceding paragraph, however, the domains of a quantum modular form and a mock modular form are notably different: quantum modular forms are defined in $\mathbb{Q}$, the set of rational numbers, as opposed to $\mathbb{U}$, the upper half of the complex plane. Under the mapping $\tau \mapsto e^{2 \pi i \tau}$, mock modular forms are defined inside the unit disk, and rational numbers correspond to roots of unity on the boundary. Despite their differing domains,

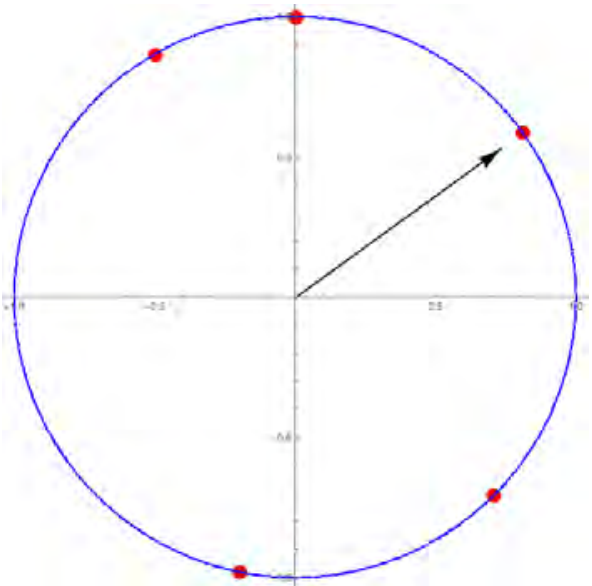

Figure 5. A radial limit

it has been a question of interest to understand the relationships between these almost symmetric functions and to farm the fruits of such relationships. See Figure 5, where a radial limit from inside the unit disk to a root of unity on the boundary is suggestively drawn.

Please join me at the 2019 AMS-MAA Joint Meetings for an accessible discussion of these and other questions surrounding symmetry, almost, guided by the topic of modular forms. The origins of these questions are rooted in the past, while some fascinating and surprising answers come from just the last $10-15$ years.

\section{Credits}

Figure 1 "Snowflake" and "Butterfly" images are courtesy of Getty Images; "Sierpinski triangle image" is by Beojan Stanislaus, distributed under a Creative Commons AttributionShare Alike 3.0 Unported license; the remaining image is courtesy of the author.

Figure 2 image is courtesy of Alexander Hulpke, distributed under a Creative Commons Attribution-Share Alike 4.0 International license.

Figure 3 image is courtesy of Jan Homann, released to the public domain.

Figure 4 is by Hermann Braus, from Anatomie des Menschen: ein Lehrbuch für Studierende und Ärzte 1921, in the public domain.

Figure 5 image is courtesy of the author.

Author photo ${ }^{\oplus}$ Amherst College.

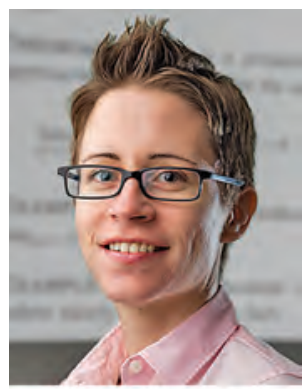

Amanda Folsom 


\section{Sailing through Data: Discoveries and Mirages}

\section{Emmanuel Candes}

NOTE. An earlier version of this text appeared in the IMS Bulletin. Seebu17etin. imstat.org/2017/05 /wa7d-1ectures-emmanuel-candes.

For a long time, science has operated as follows: a scientific theory can only be tested empirically, and only after it has been advanced. Predictions are deduced from the theory and compared with the results of decisive experiments so that they can be falsified or corroborated. This principle, formulated independently by Karl Popper and by Ronald Fisher, has guided the development of scientific research and statistics for nearly a century. We have, however, entered a new world where large data sets are available prior to the formulation of scientific theories. Researchers mine these data relentlessly in search of new discoveries, and it has been observed that we have run into the problem of irreproducibility. Consider the April 23, 2013 Nature editorial: "Over the past year, Nature has published a string of articles that highlight failures in the reliability and reproducibility of published research." The field of statistics needs to re-invent itself and adapt to this new reality in which scientific hypotheses/theories are generated by data snooping. In my lecture, I will make the case that statistical science is taking on this great challenge and discuss exciting achievements.

An example of how these dramatic changes in data acquisition have informed a new way of carrying out scientific investigation is provided by genome-wide association studies (GWAS). Nowadays we routinely collect information on an exhaustive collection of possible explanatory variables to predict an outcome or understand what determines an outcome. For instance, certain diseases have a genetic basis and an important biological problem is to find which genetic features (e.g., gene expressions or single nucleotide polymorphisms) are important for determining a given disease. Even though we believe that a disease status depends on a comparably small set of genetic variations, we have a priori no idea about which ones are relevant and therefore must include them all in our search. In statistical terms, we have an outcome variable and a potentially gigantic collec-

Emmanuel Candes holds the Barnum-Simons Chair in Mathematics and Statistics at Stanford University. His email address is candes@stanford . edu.

For permission to reprint this article, please contact: reprint -permission@ams.org.

DOI: http://dx.doi.org/10.1090/noti1770 tion of explanatory variables, and we would like to know which of the many variables the response depends on. In fact, we would like to do this while controlling the false discovery rate (FDR) or other error measures so that the results of our investigation do not run into the problem of irreproducibility. The lecture will discuss problems of this kind. We introduce "knockoffs," an entirely new framework for finding dependent variables while provably controlling the FDR in finite samples and complicated models. The key idea is to make up fake variables-knockoffs-that are created from the knowledge of the dependent variables alone (not requiring new data or knowledge of the response variable) and can be used as a kind of negative control to estimate the FDR (or any other error of type 1 ). We explain how one can leverage haplotype models and genotype imputation strategies about the distribution of alleles at consecutive markers to design a full multivariate knockoff processing pipeline for GWAS!

Some of the work I will be presenting is joint with many great young researchers including Rina Foygel Barber, Lucas Janson, Jinchi Lv, Yingying Fan, Matteo Sesia, as well as many other graduate students and post-docs, and also with Professor Chiara Sabatti, who played an important role in educating me about pressing contemporary problems in genetics. I am especially grateful to Yoav Benjamini: Yoav visited Stanford in the winter of 2011 and taught a course titled "Simultaneous and Selective Inference." These lectures inspired me to contribute to the enormously important enterprise of developing statistical theory and tools adapted to the new scientific paradigm-collect data first, ask questions later.

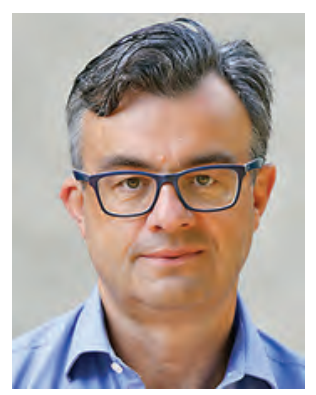

Emmanuel Candes

Credits

Author photo is courtesy of the John D. and Catherine T. MacArthur Foundation. 


\section{JMM 2019 LECTURE SAMPLER}

\section{A Dream Deferred: 50 Years of Blacks in Mathematics}

\section{Edray Herber Goins}

In January 1969, the American Mathematical Society hosted the annual Joint Mathematics Meetings in New Orleans, Louisiana. As Everett Pitcher [2, pg. 37] writes: "[T]here were 4,811 registrants, including 3,084 members of the Society. This was the time when there was a sudden increase in the number of new PhD's and the foment in the employment market as well as the pleasant location contributed to the size of this meeting." By comparison, the 2018 annual meeting in San Diego, California saw 5,572 registrants-and probably for the same two reasons.

This meeting began at the end of a tumultuous year in the United States. In December 1968, the Apollo 8 orbited the Moon and photographed "Earthrise." In November 1968, Nixon was elected President. In June 1968, Robert Kennedy was assassinated. This year was particularly poignant for African Americans: In October 1968, African American sprinters Tommie Smith and John Carlos raised black-gloved fists in solidarity with the Civil Rights Movement as they received their medals at the Summer Olympics. In April 1968, Lyndon Johnson signed the Civil Rights Act-but Martin Luther King was assassinated just the week before, sparking riots throughout the country. It should come as no surprise that African Americans felt it was time to empower Black mathematicians traveling to the 1969 Joint Math Meetings.

Just getting to the Joint Math Meetings as an African American was an adventure in itself. It was notoriously difficult for Black Americans to travel throughout the United States at the time: Jim Crow Laws made it legal for Blacks to be discriminated against for staying at hotels, using restrooms, and obtaining repairs on vehicles. So much so, that in 1936, New York City mailman Victor Green published a guidebook for African American drivers to let them know which lodgings, businesses, and gas stations would serve them along the drive. The Negro Motorist Green Book, published for 30 years, was in wide circulation through 1966. I remember growing up hearing stories from my parents about how they moved across the country from Texas

Edray Herber Goins is a professor of mathematics at Pomona College. His email address is edray.goins@pomona. edu.

For permission to reprint this article, please contact: reprint - permission@ams.org.

DOI: http://dx.doi.org/10.1090/noti1771 to California by car in the 1940s, and flipping through the Green Book when I was younger.

Several African Americans came together at the Joint Math Meetings in New Orleans to do something about the plight of Black mathematicians. Encouraged by the Civil Rights Movement and the strides gained in 1968, seventeen individuals met on January 26, 1969: James Ashley Donaldson, Samuel Horace Douglas, Henry Madison Eldridge, Thyrsa Anne Frazier Svager, Richard J. Griego, Johnny L. Houston, Curtis Jefferson, Vivienne Malone-Mayes, Theodore Portis, Arbeligic Rodriquez, Charles Smith, Robert S. Smith, Beauregard Stubblefield, Henry Taggert, Walter Talbot, Harriet R. Junior Walton, and Scott Warner Williams. These individuals formed the National Association of Mathematicians, with the charge "to promote excellence in the mathematical sciences and to promote the mathematical development of under-represented American minorities." More information can be found in Johnny Houston's book [1].

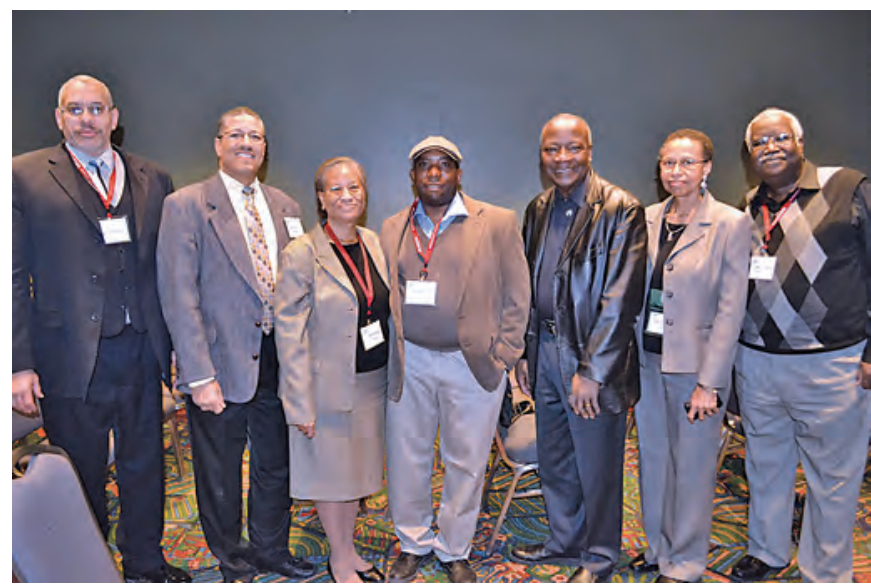

Left to Right: Leon Woodson, Duane Cooper, Roselyn Williams, Edray Goins, Johnny Houston, Sylvia Bozeman, and Robert Bozeman at the Joint Math Meetings in 2015.

Since the founding of NAM 50 years ago, there have been many changes. The National Science Foundation (NSF) states [3] that in 1968 there were 22,937 doctorates awarded in the United States across all disciplines, with 971 in mathematics and computer science. Just 9 of those went to African American mathematicians. In 2016, there were 3,957 doctorates granted in mathematics and computer science, but just 78 went to African American mathematicians. While the percentage of Blacks receiving doctorates in the mathematical sciences has doubled in the past 50 years-from $0.93 \%$ to $1.97 \%$ - this is a far cry from $12 \%$, the percentage of Blacks living in the United States.

At the 2019 Joint Mathematics Meetings, I will expand more on these stories and more. We take a tour of the mathematics done by Africans and African Americans over 
the past 50 years since the founding of NAM, weaving in personal stories and questions for reflection for the next 50 years.

\section{References}

[1] Johnny L Houston, The History of the National Association of Mathematicians (NAM): The first thirty (30) years, 19691999 (2000). MR1424534

[2] Everett Pitcher, American Mathematical Society Centennial Publications Volume I: A History of the Second Fifty Years, 1939-1988. American Mathematical Society, Providence, RI, 1988. MR1002190

[3] National Science Foundation, Science and Engineering Degrees: 1966-2010. https://nsf.gov/statistics. nsf13327/pdf/nsf13327.pdf.

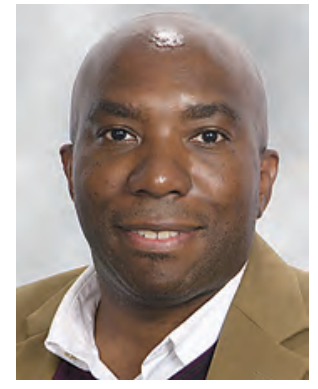

Edray Herber Goins

\section{Credits}

All article photos are courtesy of the author. 


\section{Dynamics of Systems with Low Complexity}

\section{Bryna Kra}

The simplest bi-infinite sequences in $\{0,1\}^{\mathbb{Z}}$ are the periodic sequences, where a single pattern is concatenated with itself infinitely often. At the opposite extreme are biinfinite sequences containing every possible configuration of 0 's and 1's. For periodic sequences, the number of substrings of length $n$ is bounded, while in the second case, there are $2^{n}$ substrings of length $n$. The growth rate of the possible patterns is a measurement of the complexity of the sequence, and can be used both to capture information about the sequence itself and to describe objects encoded by the sequence. Symbolic dynamics is the study of such sequences, associated dynamical systems, and their properties.

There is a simple relation between a local constraint on complexity and the global property of periodicity. If $x \in \mathcal{A}^{\mathbb{Z}}$ is a bi-infinite sequence over a finite alphabet $\mathcal{A}$, define the complexity $P_{X}(n)$ to be the number of words of length $n$ in $x$, meaning the number of different substrings of $n$ consecutive symbols appearing somewhere in $x$. A classical theorem of Morse and Hedlund [12] states that the sequence $x \in \mathcal{A}^{\mathbb{Z}}$ is periodic if and only if there exists an integer $n \geq 1$ such that $P_{X}(n) \leq n$. In other words, any sequence whose complexity grows very slowly actually has bounded complexity and is periodic.

A bi-infinite sequence naturally gives rise to an associated dynamical system. For $x=\left(x_{n}\right)_{n \in \mathbb{Z}} \in \mathcal{A}^{Z}$, define the (left) shift $\sigma: \mathcal{A}^{\mathbb{Z}} \rightarrow \mathcal{A}^{\mathbb{Z}}$ to be the map given by $(\sigma x)_{n}=x_{n+1}$. Endowing $\mathcal{A}$ with the discrete topology and $\mathcal{A}^{\mathbb{Z}}$ with the product topology, $\sigma$ is a homeomorphism from $\mathcal{A}^{\mathbb{Z}}$ to itself. If $X \subseteq \mathcal{A}^{\mathbb{Z}}$ is a closed and shift invariant set, then the resulting dynamical system $(X, \sigma)$ is a subshift. In particular, given some fixed $x \in \mathcal{A}^{\mathbb{Z}}$, by taking the closure $X$ of its orbit under the shift $\sigma$, we obtain a subshift $(X, \sigma)$. If we start with some periodic sequence $x \in \mathcal{A}^{\mathbb{Z}}$, then the resulting subshift is a periodic system. Generalizing the definition of complexity from a sequence to a system, we define the complexity $P_{X}(n)$ of the system $(X, \sigma)$ to be the number of words of length $n$ for any $x \in X$. The Morse-Hedlund Theorem can be reinterpreted in this context: if the complexity of the system

Bryna Kra is Sarah Rebecca Roland Professor of Mathematics at Northwestern University. Her email address is kra@math. northwestern. edu.

For permission to reprint this article, please contact:

reprint-permission@ams . org.

DOI: https://doi.org/10.1090/noti/1779
$(X, \sigma)$ satisfies $P_{X}(n) \leq n$ for some integer $n \geq 1$, then $(X, \sigma)$ is a periodic system. At the opposite extreme, if we start with a bi-infinite sequence $X$ that contains all words of all lengths and form the naturally associated dynamical system $(X, \sigma)$, we obtain the full shift, meaning that $X=\mathcal{A}^{\mathbb{Z}}$ and $P_{X}(n)=|\mathcal{A}|^{n}$ for all $n \geq 1$.

The exponential growth rate of the complexity function $P_{X}(n)$ is one way to define the entropy of the system. This leads to a natural dichotomy between positive entropy, meaning exponential growth rate of the complexity, and zero entropy, meaning subexponential growth. Symbolic systems with positive entropy are often viewed as more random, typically exhibiting some sort of hyperbolic behavior, many invariant measures, and large automorphism groups (see for example $[1,11]$ ). On the other hand, symbolic systems with zero entropy are viewed as deterministic and so simpler in some way. In spite of the restrictions imposed by zero entropy, many basic questions about such systems remain open. Even when placing strong constraints on the complexity of the system, easily formulated questions remain intractable.

It follows from the Morse-Hedlund Theorem that the first interesting case is a subshift $(X, \sigma)$ satisfying

\section{$P_{X}(n) \geq n+1$ for all $n \geq 1$.}

If the complexity of the subshift has linear growth, we have a fairly complete picture of the constraints that this assumption places on the system. For example, consider the automorphism group $\operatorname{Aut}(X, \sigma)$, meaning the collection of all homeomorphisms $\phi: X \rightarrow X$ that commute with $\sigma$. If $(X, \sigma)$ has linear complexity, then its automorphism group has a simple structure: every finitely generated subgroup of $\operatorname{Aut}(X, \sigma)$ is virtually $\mathbb{Z}^{d}$ for some $d$ that depends on the linear growth rate of the complexity, and further constraints hold with stronger dynamical assumptions on the system (see $[3,10])$. Linear growth also places constraints on the number of ergodic nonatomic measures that can be supported on a subshift, and in [8] we show that there can be at most finitely many such measures. These results have various applications outside of dynamics, leading to combinatorial and number theoretic results related to the complexity of particular systems.

However, by even raising the complexity to quadratic growth, the situation becomes more complicated, with only partial descriptions $[2,6,7,9]$ of the group of automorphisms, incomplete descriptions of the collection of invariant measures, and difficulties in applying the results. In an interesting twist, it turns out that some of these results can be approached by going beyond the one dimensional setting and considering $\mathcal{A}^{\mathbb{Z}^{d}}$ for some integer $d \geq 2$. Again, given a multi-dimensional configuration, there is a naturally associated dynamical system, but with $d$ commuting shifts instead of a single one. The definitions of complexity and periodicity can be extended, but there are 
numerous ways in which one can do so, introducing new complications. As an example, it is not clear how to generalize the basic result of Morse and Hedlund to give a relation between complexity and periodicity. Partial results are known $[4,5,13,14]$, and while some of these can be used to further our understanding of one dimensional subshifts, we are far from having a complete understanding of these settings. We focus on the many open questions in this area, both within dynamics and in terms of applications.

\section{References}

[1] Boyle $M$, Lind $D$, Rudolph $D$, The automorphism group of a shift of finite type. Trans. Amer. Math. Soc. 306(1) (1988), 71-114. MR927684

[2] Cyr V, Franks J, Kra B, Petite S, Distortion and the automorphism group of a shift. To appear, J. Mod. Dyn.

[3] Cyr V, Kra B, The automorphism group of a shift of linear growth: beyond transitivity. Forum Math. Sigma 3 (2015), e5, 27 pp. MR3324942

[4] Cyr V, Kra B, Nonexpansive $\mathbb{Z}^{2}$-subdynamics and $\mathrm{Ni}$ vat's conjecture. Trans. Amer. Math. Soc. 367 (2015), no. 9, 6487-6537. MR3356945

[5] Cyr V, Kra B, Complexity of short rectangles and periodicity. European J. Combin. 52 (2016), part A, 146-173. MR3425972

[6] Cyr V, Kra B, The automorphism group of a shift of subquadratic growth. Proc. Amer. Math. Soc. 144 (2016), no. 2, 613-621. MR3430839

[7] Cyr V, Kra B, The automorphism group of a minimal shift of stretched exponential growth. J. Mod. Dyn. 10 (2016), 483-495. MR3565928

[8] Cyr V, Kra B, Counting generic measures for a subshift of linear growth. J. Eur. Math. Soc. Electronically published on October 12, 2018. doi: 10.4171/JEMS/838. To appear in print.

[9] Cyr V, Kra B, The automorphism group of a shift of slow growth is amenable. To appear, Ergodic Theor Dynam. Systems

[10] Donoso S, Durand F, Maass A, Petite S, On automorphism groups of low complexity subshifts. Ergodic Theory Dynam. Systems 36 (2016), no. 1, 64-95. MR3436754

[11] Hedlund, GA, Endomorphisms and automorphisms of the shift dynamical system. Math. Systems Theory. 3 (1969), 320-375. MR0259881

[12] Morse M, Hedlund GA, Symbolic dynamics II. Sturmian trajectories. Amer. J. Math. 62 (1940) 1-42. MR0000745

[13] Quas A, Zamboni L, Periodicity and local complexity. Theoret. Comput. Sci. 319 (2004), no. 1-3, 229-240. MR2074955

[14] Sander, JW, Tijdeman R, The complexity of functions on lattices. Theoret. Comput. Sci. 246 (2000), no. 1-2, 195-225. MR1780238

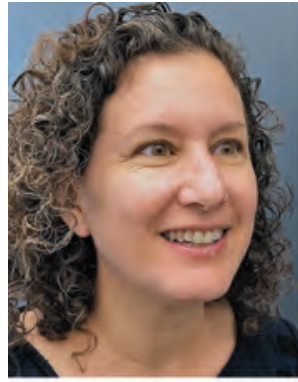

Bryna Kra

Credits

Author photo is by Jared Wunsch. 


\section{A Mathematical Journey of Culture, Community, and Collaboration}

\section{Pamela E. Harris}

It wasn't until the last year of my graduate program that I met another Latina PhD mathematician. Before this I thought that I may be the only Latina working on a PhD in the mathematical sciences. Of course this was silly, as I could have simply searched the words "Latinas in math" to discover Ruth Gonzalez, the first US born Hispanic woman who earned a PhD in mathematics. The year? 1986-during my lifetime.

As a first generation college student and a dreamer, the experience of not knowing people of similar cultural and socioeconomic backgrounds working in academia affected my confidence and belief that I could become a mathematician. Not feeling part of the mathematical community left me isolated and unsure of my abilities to succeed in this field. However, this isolation dissipated through my participation in conferences and programs designed to establish research collaborations, especially for scholars of color. Moreover, these experiences positively impacted my goals as an educator and have shaped my desire to build collaborative learning communities within my classroom.

In this talk I will share some of my mathematical journey and discuss programs and opportunities that helped my development as a mathematician and educator, while presenting ideas for how we can duplicate these experiences in smaller scales. I will also discuss ways to establish and maintain active collaborations that are not only mathematically productive, but are firmly based on respect, appreciation, and support. Lastly, I will share pedagogical techniques aimed to instill mathematical confidence in all students, as well as how to build learning and research communities to help develop a student culture of continuous improvement and collective responsibility.

Pamela E. Harris is assistant professor of mathematics at Williams College. Her email address is peh2@wi 11 iams. edu.

For permission to reprint this article, please contact: reprint -permission@ams.org.

DOI: http://dx.doi.org/10.1090/noti1772

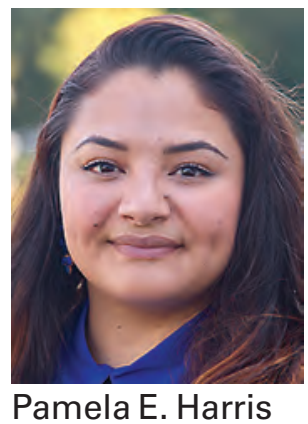

Credits

Author photo is courtesy of Cesar E. Silva. 


\section{The Roaring Twenties in American Mathematics}

\section{Karen Hunger Parshall}

In December 1918, Edward Van Vleck was "crazy to get back into real scientific work." The University of Wisconsin mathematician had turned fifty-four just months after the United States had entered World War I in 1917 and had engaged in the war effort as an instructor for the Student Army Training Corps (SATC) on his home campus in Madison. With his usual nine hours of teaching a week augmented by two additional four-hour classes of freshman algebra targeted at SATC students, his "war work," not surprisingly, had "absorbed all of [his] spare time and energy." He had been completely diverted from the analytical research that he had been faithfully pursuing since his days in Göttingen as a doctoral student of Felix Klein.

Van Vleck was, in some sense, a member of the "first generation" of research mathematicians in the United States. Although he had done graduate work at the Johns Hopkins University before earning his Göttingen PhD, he, like so many other American mathematical aspirants born in the 1860s, had recognized that the kind of training he sought was largely unavailable in the United States in the early 1890s. He thus went abroad and returned with a personal mathematical research agenda as well as a dual sense of his academic mission. He was a teacher of undergraduate as well as graduate students, but he was also an active researcher. After 1904 and thanks to its then president, the geologist Charles Van Hise, the University of Wisconsin to which Van Vleck had moved in 1906 was also coming to share this ethos. It was one of the state universities that had begun to respond to changes in American higher education under way at least since 1876 with the founding of Hopkins in Baltimore. In fits and starts, other institutions followed suit into the opening decades of the twentieth century.

In many ways, World War I had served as a wake-up call to those in academe but, perhaps more importantly, to others in newly created philanthropies as well as to some within the federal government. They had begun to recognize the value of original research for the welfare of the nation; they increasingly saw the need to support research financially. Savvy university administrators witnessed and steadily responded to this trend over the course of the 1920s and 1930s. They followed the money. Maybe the philanthropies were on to something. Maybe research should be more vigorously encouraged within the universities. Maybe faculties should be formed and sustained on the basis of research productivity and graduate training, first, and undergraduate teaching, second.

The war had also served as a break in business as usual. In its aftermath, there was a sense within the scientific community more broadly, but within the mathematical community, in particular, of entering into "a new era in the development of our science." "Every nerve should be strained to get our research back on its feet," in the view of Brown University's Roland Richardson. He was apparently not alone in this conviction. He and other American mathematicians poured themselves into their work in the 1920s, but what did that mean? What were their main research interests? Where were those interests fostered? What, in short, was the lay of the American mathematical research landscape in the 1920s? These are the questions that this talk will explore.

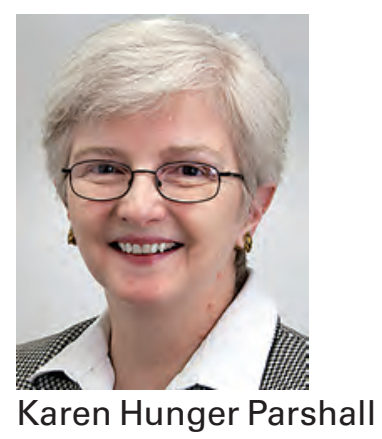

Credits

Author photo courtesy of Bryan Parsons.

Karen Hunger Parshall is a professor of history and mathematics at the University of Virginia. Her email address is khp3k@vi rginia. edu.

For permission to reprint this article, please contact: reprint -permission@ams.org.

DOI: http://dx.doi.org/10.1090/noti1773 


\section{JMM 2019 LECTURE SAMPLER}

\section{A Seat at the Table: Equity and Social Justice in Mathematics Education}

\section{Talithia Williams}

As mathematics educators, we often pride ourselves in our ability to communicate the joy and beauty of mathematics to our students. To watch a student's face light up over a clever proof or robust statistical model still gives me goose bumps. And it's often those students-the ones that are bright eyed and eager-that I invite to the metaphorical mathematical table: sending them REU announcements, financial analyst summer opportunities, or graduate school suggestions. Over time, I realized that I wasn't allowing my students to engage in mathematics in ways that were meaningful to them. Although well intentioned, my classrooms were far from "mathematically inclusive."

In recent years, I've learned to build upon my students' identity and lived experiences by incorporating rigorous, relevant mathematical experiences into the classroom environment. These experiences not only provide deep mathematical learning, but also give students the mathematical knowledge to advocate for justice in our communities. By creating inclusive classrooms and promoting classroom experiences that are equitable, we can change the culture of mathematics. In this talk, I'll share the tangible ways in which we can invite our students to have a seat at the mathematical table and in doing so, work to broaden participation in mathematics. I'll also highlight the work I've been doing in our community with underrepresented girls and parents to promote mathematical learning and achievement.

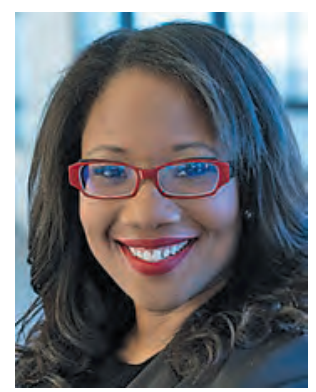

Talithia Williams

Talithia Williams is associate dean for research and experiential learning and an associate professor of mathematics at Harvey Mudd College. Her email address is twi11iams@g.hmc.edu.

For permission to reprint this article, please contact: reprint -permission@ams.org.

DOI: http://dx.doi.org/10.1090/noti1774

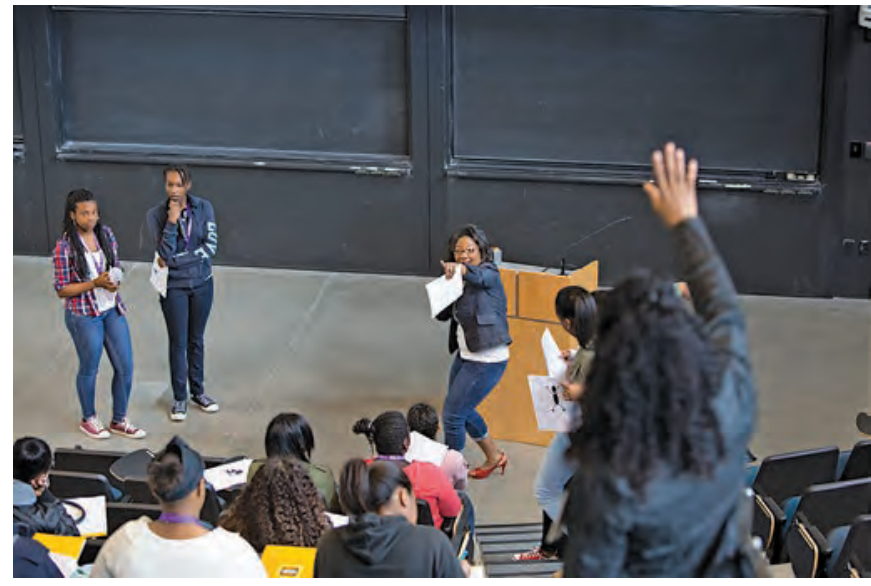

Williams with local middle and high school girls at a Harvey Mudd College mathematics conference.

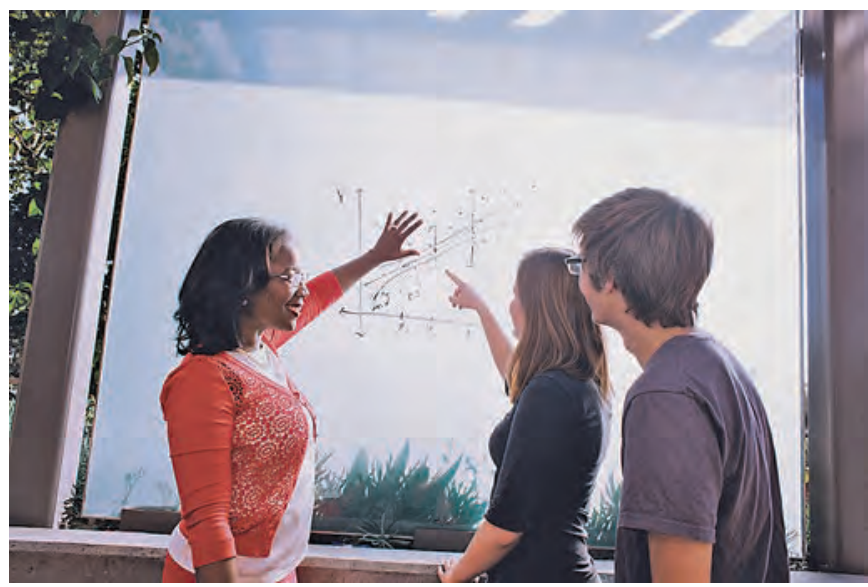

Williams working with Harvey Mudd College students.

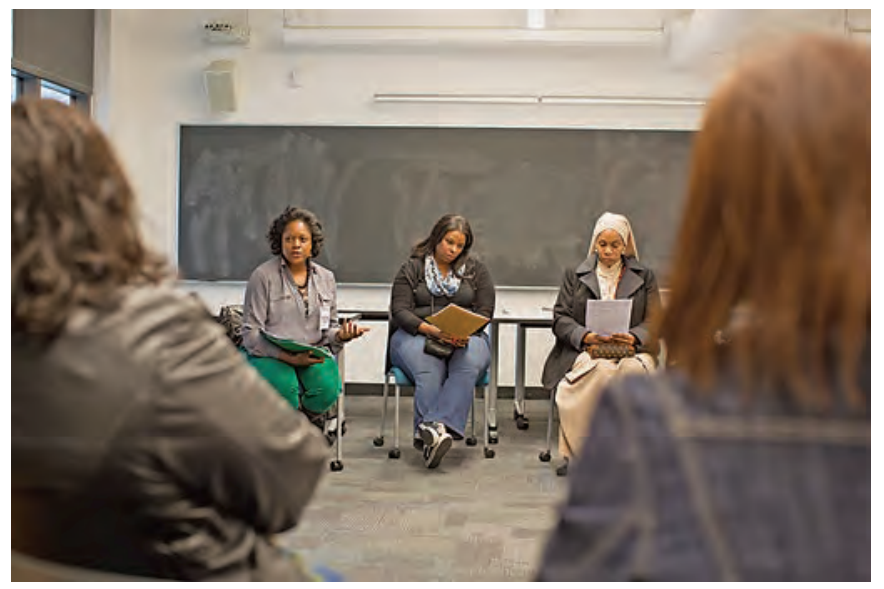

A conference session conducted for parents to provide resources and support for mathematics success.

\section{Credits}

Author photo is courtesy of Harlem Logan.

All other article photos are courtesy of Harvey Mudd College. 


\section{On Torsion Subgroups in Class Groups of Number Fields}

\section{Lillian B. Pierce}

At the turn of the 19th century, Gauss was interested in the problem of representation by quadratic forms. The germ of this problem can be traced back as far as Diophantus, nearly 2000 years ago. But let us start for concreteness with a statement by Fermat around the middle of the 17th century that an odd prime can be written as a sum of two squares if and only if it is congruent to 1 modulo 4 . Since the square of an integer is either congruent to 0 or 1 modulo 4, the "only if" part of this claim is simple to prove, but the "if" part is more tricky. It was one hundred years after Fermat's statement that Euler provided a proof, and the problem then came to be situated within a broader setting: studying when an integer $m$ is represented by a binary quadratic form $Q(x, y)=a x^{2}+b x y+c y^{2}$, that is, whether there exist integer solutions $x, y$ to the equation $Q(x, y)=m$.

In fact, it is natural to sort binary quadratic forms by discriminant $\left(b^{2}-4 a c\right.$ in this notation). Then one can ask: given an integer $m$, is there a form of discriminant $D$ that represents $m$ ? If so, which forms of discriminant $D$ represent $m$ ? Twenty-five years after Euler, Lagrange gave an answer to the first, simpler question: one simply needs to test whether $D$ is a quadratic residue modulo $4 m$, that is, whether there exists an integer $x$ such that $D \equiv x^{2}$ modulo $4 \mathrm{~m}$. But this left open the question of which forms of discriminant $D$ represent $m$, and here is where Gauss took up the problem.

Gauss clarified that you can dissect the set of forms of discriminant $D$ into equivalence classes under $\mathrm{SL}_{2}(\mathbb{Z})$ changes of variable; forms in the same equivalence class represent the same integers. Moreover, for each $D$ there are finitely many such equivalence classes; the number of such classes is the class number associated to $D$. The question is now which (if any) equivalence classes of forms of discriminant $D$ represent $m$ ?

Given $D<0$, Gauss constructed a finite set of test functions (real characters with moduli determined by the prime divisors of $D$ ), and showed how to use these test functions

Lillian B. Pierce is an associate professor of mathematics at Duke University. Her email address is pi erce@math. duke. edu.

For permission to reprint this article, please contact:

reprint-permission@ams .org.

DOI: https://doi.org/10.1090/noti/1777 to create a "fingerprint" for each quadratic form of discriminant $D$ (by evaluating the characters at an integer represented by the form). If there are $t$ test functions, this fingerprint is a sequence of $t$ values of $+1,-1$ whose product is +1 . Forms in the same equivalence class have the same fingerprint. But it can also happen that different equivalence classes may have the same fingerprint.

Gauss called these new coarser clusters, comprised of forms sharing a fingerprint, the genera of the discriminant $D$. He showed that there are $2^{t-1}$ distinct genera, and each genus contains the same number of equivalence classes; hence we arrive at the remarkable fact that $2^{t-1}$ must divide the class number. How big is $t$ ? It turns out that $t$ is the number of distinct prime divisors of $D$ (here for simplicity we assume $D$ is a fundamental discriminant, which means it is square-free, up to certain powers of 2). Gauss had revealed (one might say accidentally) a remarkably simple criterion for when the class number of $D$ is divisible by powers of 2 . (The case $D>0$ is similar.)

In terms of studying the divisibility properties of class numbers, this is the worm that opened the can. (Although it left open the representation question-we know how to test which genera represent a given integer $m$, but still not which equivalence class or classes within a genus represent $m$. If we think about the collection of genera as a nest of eggs, and each equivalence class as a yolk, the ambiguity arises when each of the eggs has more than one yolk.)

What about divisibility of the class number by 3 ? by 5 ? We still don't understand these questions nearly as well. Even questions about the size of class numbers remain difficult. Gauss did a remarkable number of computations by hand (for example computing the class number for each discriminant between $-9,000$ and $-10,000$ ), which led to beautiful predictions, such as that there should only be finitely many $D<0$ with a given class number, while there should be infinitely many $D>0$ with class number 1. Alongside his predictions, he remarked, "Demonstrationes autem rigorosae harum observationum perdifficiles esse videntur." ${ }^{\prime \prime}$ Among the many things that Gauss was right about, he seems to have been right about this; celebrated work has shown the first statement to be true, while the second remains a mystery.

In modern terms, Gauss was studying the class group (with cardinality being the class number) of quadratic fields $\mathbb{Q}(\sqrt{D})$, for a positive or negative integer $D$. More generally, given any finite degree field extension $K$ of $\mathbb{Q}$, we can study the class group $\mathrm{Cl}_{K}$, which is defined to be the quotient group of the fractional ideals of the ring of integers of $K$ by the principal ideals. In each case, the class group $\mathrm{Cl}_{K}$ is a finite abelian group, whose cardinality is called the class number. Class groups are central objects in number

\footnotetext{
${ }^{1}$ Translated in [GSS07, p.13] as "However, rigorous proofs of these observations appear to be difficult."
} 
theory, and questions about the divisibility properties of class numbers fit into a broader theme of questions about the structure of class groups of fields as the fields vary over a family of interest.

This talk will focus on the size of torsion subgroups in class groups. If we fix a prime $\ell$, how many elements of order $\ell$ ( $\ell$-torsion elements) can exist in a class group $\mathrm{Cl}_{K}$ for a particular field $K$ ? Can we learn more if we work on average as $K$ varies over an appropriately defined family of fields? This talk will survey recent progress on these questions, and how they relate to other well-known conjectures in number theory, such as fundamental questions on counting number fields.

\section{References}

[GSS07] Catherine Goldstein, Norbert Schappacher, and Joachim Schwermer, The Shaping of Arithmetic after C. F. Gauss's Disquisitiones Arithmeticae. Springer-Verlag, 2007. MR2308276

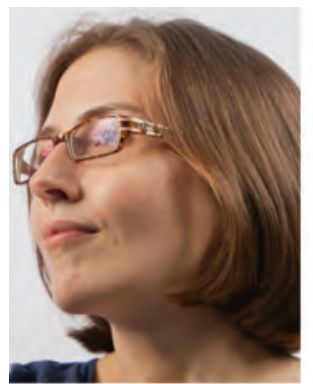

Lillian B. Pierce

\section{Credits}

Author photo is courtesy of Lillian B. Pierce. 


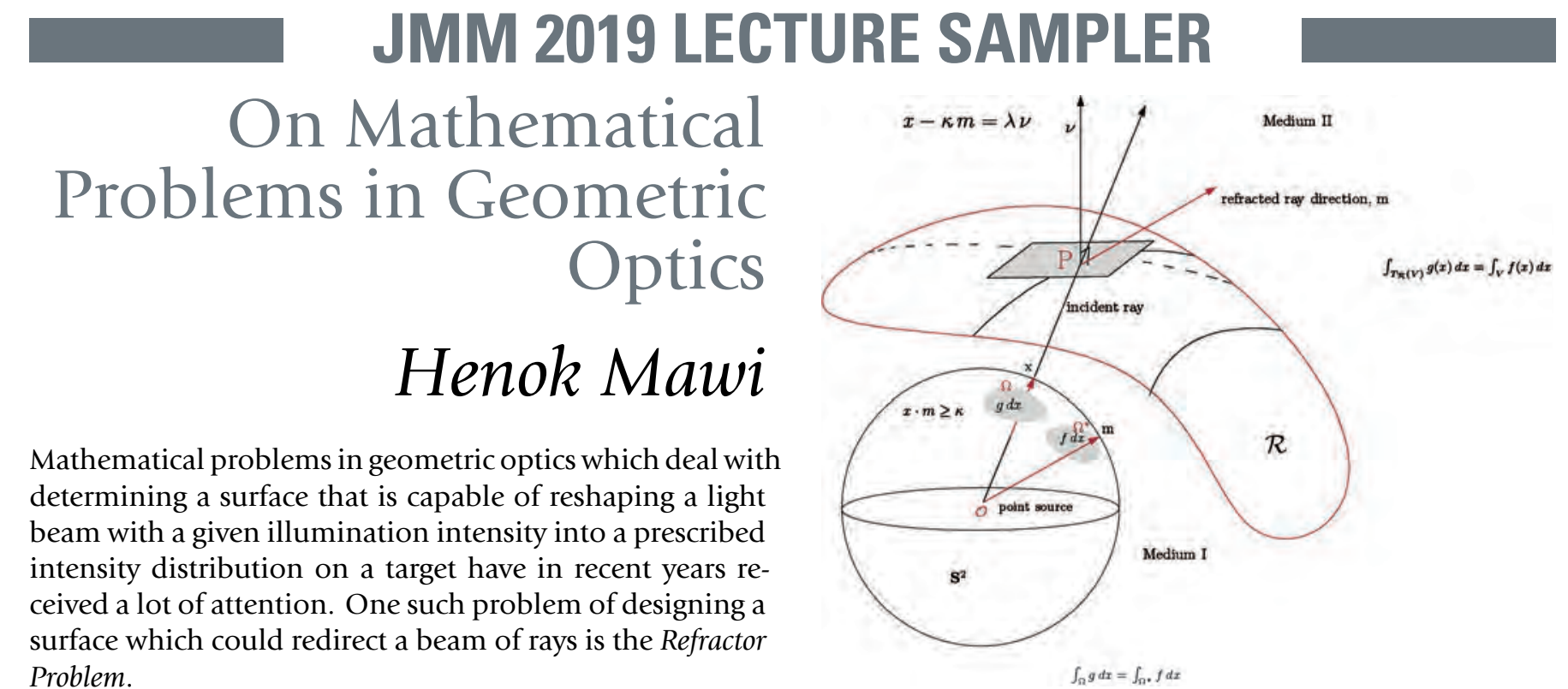

In a version of this problem, we are given two media of propagation of light; medium I and medium II and a monochromatic light emanating from a point source located at some point, say the origin, $O \in \mathbb{R}^{3}$ in medium I, see Figure 1. The two media being considered have different optical properties that force the given light to travel with different velocities. As a result, bending of light rays, also known as refraction happens as a ray of light traverses an interface between medium I and medium II. From the point source, the light beam shines through an aperture $\Omega \subset S^{2}$, the unit sphere in $\mathbb{R}^{3}$, with input intensity given by a positive density function $g$ which is integrable on $\Omega$. We are also given $\Omega^{*} \subset S^{2}$ and positive density function $f \in L^{1}\left(\Omega^{*}\right)$ which models the output intensity.

The objective is then to find an interface (lens) $\mathcal{R}$ given by the graph of a radial function

$\rho$ as $\mathcal{R}=\{\rho(x) x: x \in \Omega\}$

between media I and II, such that a ray emitted from the point $O$ with a direction $x \in \Omega$ and input density $g$ is refracted by the surface $\mathcal{R}$ into a direction $m \in \Omega^{*}$ as it proceeds into media II and the illumination intensity received is given by the density function $f$. It is natural to assume that $g$ and $f$ satisfy the energy conservation condition

$$
\int_{\Omega} g d x=\int_{\Omega^{*}} f d x
$$

with $d x$ being the surface measure on $S^{2}$.

The input and output intensities and directions of radiation are prescribed a priori and so the problem of recovering the surface $\mathcal{R}$ is an inverse problem. The mathematical

Henok Mawi is an assistant professor of mathematics at Howard University. His email address is henok. mawi@howard.edu.

For permission to reprint this article, please contact: reprint-permission@ams.org.

DOI: https://doi.org/10.1090/noti/1778

Figure 1. Figure 1. Far Field Refractor Problem

formulation of this problem is based on a systematic application of the laws of geometrical optics and laws of energy conservation.

Indeed, if a ray of light traveling through medium I in the direction $x \in S^{2}$ traverses an interface $\mathcal{R}$ at point $P$ and continues to propagate in medium II in the direction $m \in S^{2}$ (see Figure 1) then from the vector form of the law of refraction (Snell's law) we obtain

$$
x-\kappa m=\lambda v
$$

where $v$ is the normal to the surface $\mathcal{R}$ at $P$ pointing into medium II, $\kappa$ is the refractive index and $\lambda=F(x, v, \kappa)$. By this relation, if appropriate geometric conditions are imposed on $\Omega$ and $\Omega^{*}$, the surface $\mathcal{R}$ generates a set valued map called the tracing mapping, $T_{\mathcal{R}}: \Omega^{*} \rightarrow \Omega$ which assigns to $m \in \Omega^{*}$ the set $T_{\mathcal{R}}(m)$ of directions $x \in \Omega$ which refract off $\mathcal{R}$ in the direction of $m$.

A solution $\mathcal{R}$ to the refractor design problem not only sends $\Omega$ to $\Omega^{*}$ via refraction, but also fulfills the energy redistribution condition. More precisely, we define a surface $\mathcal{R}$ to be a weak solution to the refractor problem with emitting illumination intensity $g$ on $\Omega$ and prescribed refracted illumination intensity $f$ on $\Omega^{*}$ if for any Borel set $V \subset \Omega^{*}$,

$$
\left\{\begin{array}{l}
\int_{T_{\mathcal{R}}(V)} g(x) d x=\int_{V} f(x) d x \\
T_{\mathcal{R}}\left(\Omega^{*}\right)=\Omega .
\end{array}\right.
$$

Interest to investigate several theoretical (existence and regularity of $\mathcal{R}$ ) and computational aspects of the above or similar problems in geometric optics has risen mainly because the techniques used in their analysis interweave 


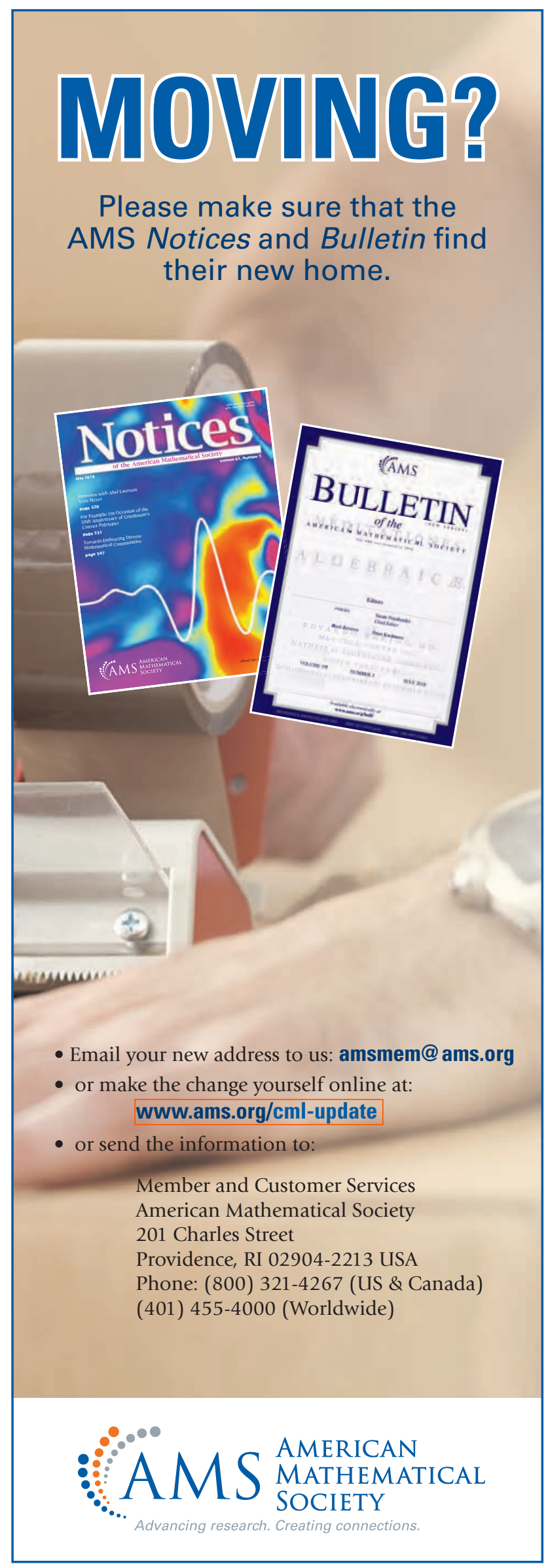

ideas from the mathematics of optimal transportation theory, calculus of variations, and nonlinear partial differential equations of Monge-Ampère type.

In this talk I hope to present an overview of these problems and describe some recent results regarding the refractor problem.

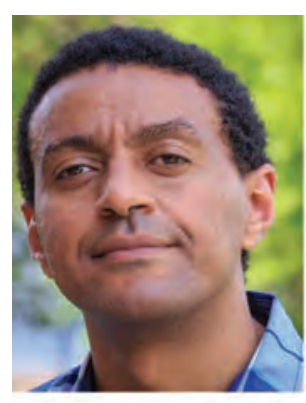

Henok Mawi

\section{Credits}

Figure 1 is courtesy of Henok Mawi.

Author photo is courtesy of Elena Maximova. 


\section{OPEN MATH NOTES}

A repository of freely downloadable mathematical works in progress hosted by the American Mathematical Society as a service to researchers, teachers, and students.

These draft works include course notes, textbooks, and research expositions in progress. Visitors are encouraged to download and use these materials as teaching and research aids and to send constructive comments and suggestions to the authors.

Some popular notes include:

- Linear Algebra by Terence Tao

- Introduction to Complex Analysis by Michael Taylor

- Topology of Numbers by Allen Hatcher

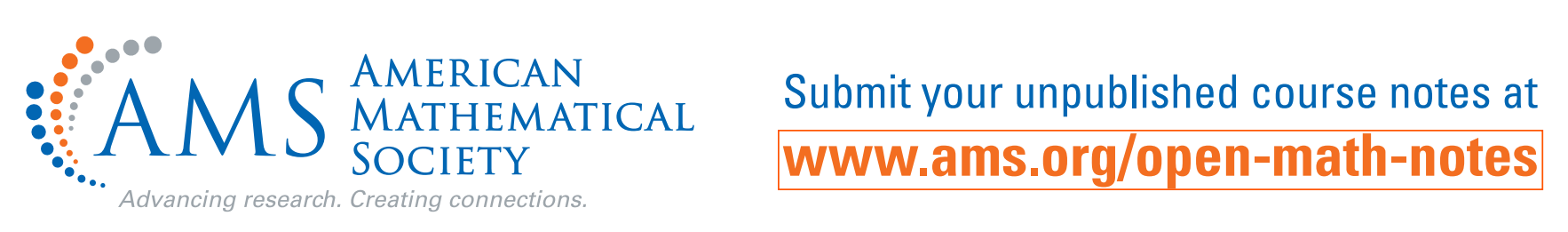




\section{CONGRESSIONAL BRIEFINGS}

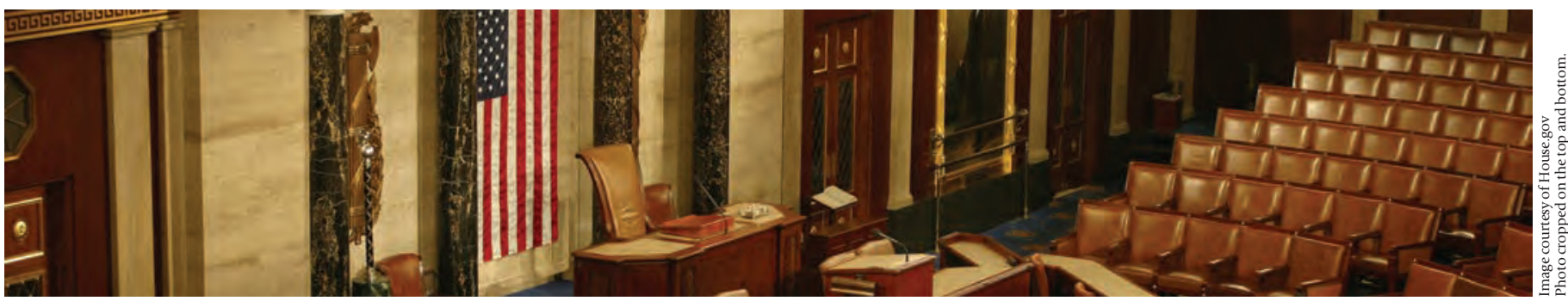

\section{Math Societies to Lawmakers: This Is Why You Should Fund Basic Research}

\section{Sophia D. Merow}

\section{Biannual Congressional Briefings}

The American Mathematical Society (AMS)_-jointly with the Mathematical Sciences Research Institute (MSRI) - sponsors two congressional briefings per calendar year. These briefings provide an opportunity for the mathematics community to impart information to policymakers and, in particular, to tell compelling stories of how federal investment in basic research in mathematics yields rich dividends.

May 22, 2018 found the far-flung AMS leadership gathered on Capitol Hill in a room secured for their use by the office of Senator Chuck Schumer (D-NY). President Kenneth A. Ribet had traveled to DC from California, President Elect Jill C. Pipher from Rhode Island, Vice President Ken Ono from Georgia. Associate Executive Director Karen Saxe, head of the Society's Office of Government Relations, had had a shorter commute, from the division's digs on Eighteenth Street.

Erik Demaine, professor of computer science and engineering at MIT, was there in the Russell Senate Office Building, too. He wore a suit and had scissors.

By noon, when Saxe stepped to the lectern to kick off a briefing titled "Origami Meets Math, Science, and Engineering," more than 50 people had claimed boxed lunches

Sophia D. Merow is special projects editor and Notices assistant. Her email address is merow. notices@gmai 1 . com. For permission to reprint this article, please contact: reprint-permission@ams .org. DOI: http://dx.doi.org/10.1090/noti1767 and arrayed themselves around the room's circular tables. Besides AMS brass, MSRI's Outreach Director Kirsten Bohl and Director David Eisenbud, and math enthusiasts young and old, the audience included staffers from the offices of Senators Amy Klobuchar (D-MN), Bill Nelson (D-FL), and Chuck Schumer, and Representatives Michael Capuano (D-MA), Mike Kelly (R-PA), Jerry McNerney (D-CA), Grace Meng (D-NY), Nancy Pelosi (D-CA), and Jacky Rosen (DNV). The US Senate Committee on Energy and Natural Resources, the Senate Democratic Policy Committee, the House Appropriations Committee, the National Science Foundation, and the Simons Foundation also had affiliates on hand.

"You're in for a real treat today," Saxe told the crowd.

Taking the mic after Saxe and California congressman Jerry McNerney (see sidebar, p. 104), MSRI director David Eisenbud explained the purpose of the briefing. He underscored the importance of basic research—and thus funding 
for it-and the impossibility of predicting which lines of inquiry will lead to future breakthroughs.

"Who knew," he asked rhetorically, "that the ancient art in Japan of folding cranes would turn out to be something that's important in engineering?"

In the presentation that followed, Demaine regaled his audience with example after example of origami-related work with real-world relevance.

Consider, for instance, Origamizer, the freeware collaboration of Demaine and Tomohiro Tachi that generates a crease pattern to reproduce any $3 \mathrm{D}$ shape in origami. It seems like a boon only to those few keen to craft from a single square of paper a Jedi or a samurai helmet beetle, but the Origamizer algorithm in fact invites far broader application: It suggests a new approach to manufacturing. Sheets of steel or cardboard or plastic are easier and cheaper to ship than hollow 3D forms, after all, and with creases laser-scored pre-shipment...

"I imagine a future where, when you go to buy a chair, it comes flat and you pop it out and fold it," Demaine said. "It's sort of an extreme form of IKEA ${ }^{\circledR}$."

And deployable origami structures have uses in environments more extreme than a family room or den. Telescope lenses can be folded to fit into space vehicles for transport, arterial stents inserted compactly then expanded once placed. A team at Brigham Young University incorporated origami's Yoshimura crease pattern into its design for a portable ballistic barrier to shield emergency personnel from gunfire (see bit.7y/2LOheN6).

Demaine's discussion of linkages, which he called a "sort of a one-dimensional type of folding," highlighted another potentially life-saving application. A protein is essentially a complicated linkage, and a paper co-authored by Demaine [1] leveraged this similarity to hypothesize the algorithm biology follows to fold proteins. Such insights into protein production could inform the development of new drugs, Demaine explained, speeding the eradication of disease.

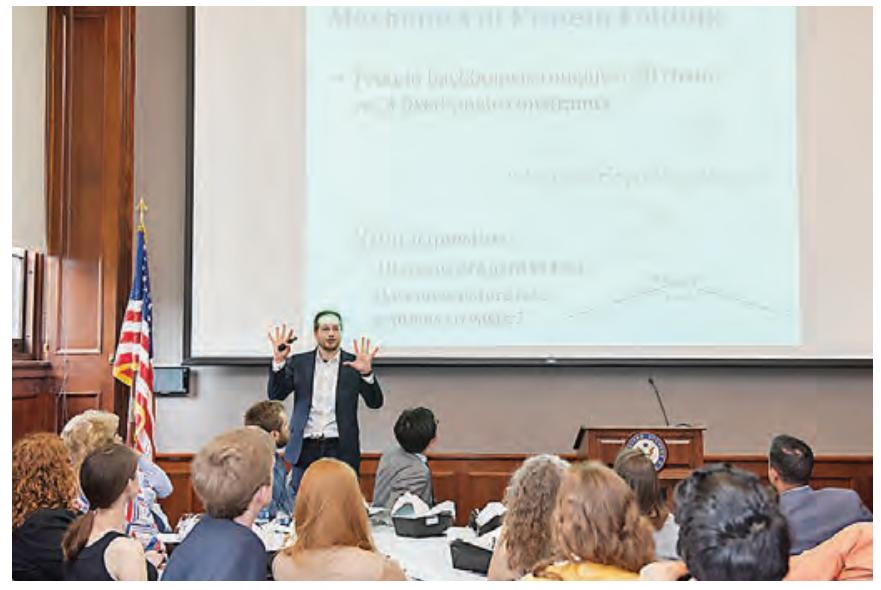

Erik Demaine explains that solving problems about linkages could help unravel the mystery of how proteins fold.
"This is why you should fund basic research," he said, "so that we can understand the mathematics and the geometry of how linkages fold. The hope is we can then solve how proteins fold and solve world health."

Demaine not only repeatedly plugged the importance of basic research, but refrained (per instructions from his AMS/MSRI coaches) from delving deeply into the mathematics underlying the wonders-the folding microscopes and microscale ingestible robots and programmable matter-he described. When he did mention a theoremthe "Fold \& One Cut Theorem"- - he used his scissors to illustrate.

Demaine flourished a piece of paper, folded it flat, and-with a single straight cut-made the (polygonal) outline of a swan.

"Okay, you're not impressed," he deadpanned. "I'll do another one. Here we have a-one straight cut, and when we unfold-we get a little angelfish."

Houdini did this trick, Demaine said, and a perhaps apocryphal story has Betsy Ross speedily populating star-spangled banners, one snip per five-pointed star. It turns out that every pattern (plane graph) of straight-line cuts can be produced in this manner, by folding flat and making one complete straight cut (see erikdemaine.org/ foldcut).

"This is a problem motivated by magic, essentially," Demaine said. "This is a result we thought would be useless. But it turns out to save lives. It gives you a way to fold an airbag flat." 1

In the Q\&A that followed Demaine's prepared remarks, he fielded questions about the potentially fruitful intersection of origami and materials science, about the elaborate corrugations of poppy flowers. A Congressional staffer wondered whether Demaine has considered possible negative outgrowths of the work he surveyed, self-deploying weapons, perhaps, or manufacturing jobs dwindling in the face of fully automated assembly lines.

"I think almost any technology can be used for positive and negative applications," Demaine answered. "My hope is that the positive applications will dwarf the negative ones. It's hard to prevent negative applications, but you do the best you can."

\footnotetext{
${ }^{1}$ The airbag simulation Demaine showed during his presentation is actually not an implementation of his fold-and-one-cut work; German firm EASi Engineering (now carhs) in fact used Robert Lang's TreeMaker, (see bit. $1 \mathrm{y} / 2 \mathrm{mG} 6 \mathrm{rHX}$ ), to devise a systematic way to flatten an airbag. Lang notes that TreeMaker's algorithm and Demaine's One Cut algorithm "solve very similar problems, of folding a shape so that all of its edges lie on a straight line, and for certain classes of polygon, they give exactly the same answer." But neither algorithm enables the accident protection airbags provide. "The method never made its way into the car development as today real airbags are folded and mostly tucked by machine, and those machines don't care about a mathematically correct folding," says carhs Managing Director Rainer Hoffmann. "The textile nature of the material allows enough crumpling to get the material into the box."
} 


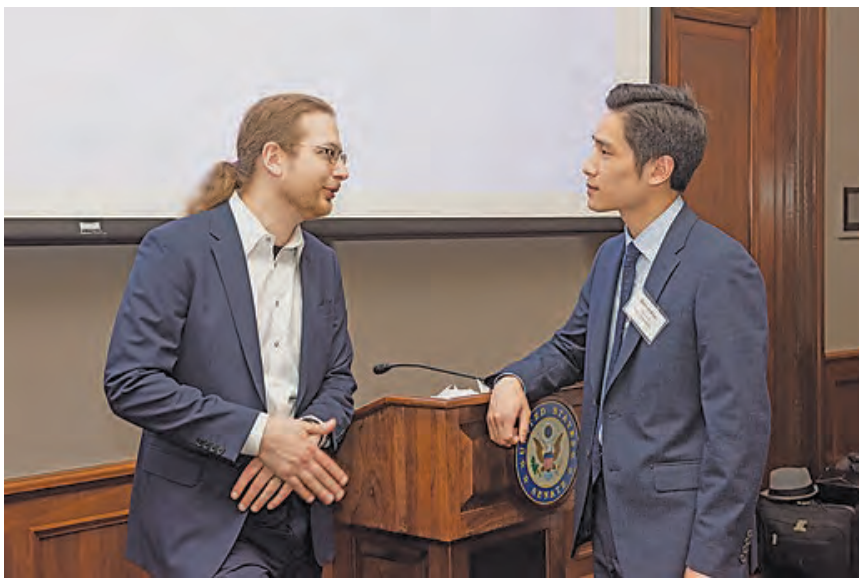

Erik Demaine talks after the briefing to Daniel Kim from the office of Representative Mike Kelly (R-PA).

When a self-described "old budget officer" asked about the current state of funding for work like Demaine's, Demaine replied that, in these early days of origami technology, most of his projects fall under the 'basic research' umbrella.

"It's hard to go to the more applied funding sources and say, 'We're going to solve cancer,'" he explained. "We don't know what we're going to solve."

The briefing's final word went to the Honorable Bart Gordon, a long-time supporter of science who represented Tennessee's $6^{\text {th }}$ District in Congress for more than a quarter of a century.

"The first person who can make their lunchbox into a robot will be able to take the scissors home," he joked, but then grew serious.

The private sector is unlikely to fund basic research, Gordon observed, given the uncertainty of it generating near-term profits. "Let the private sector do a lot of the applied research," he said. "But the federal government has got to do the basic research. So please carry that message."

Help spread the word about the importance of funding basic research:

- Write a letter to your senator or representative (to get started, see https://bit.7y/2G1Vav1).

- Write an op-ed for a local publication.

- Organize a talk at your institution about the unexpected utility of basic math. Invite your representative and work with your institution's government relations office.

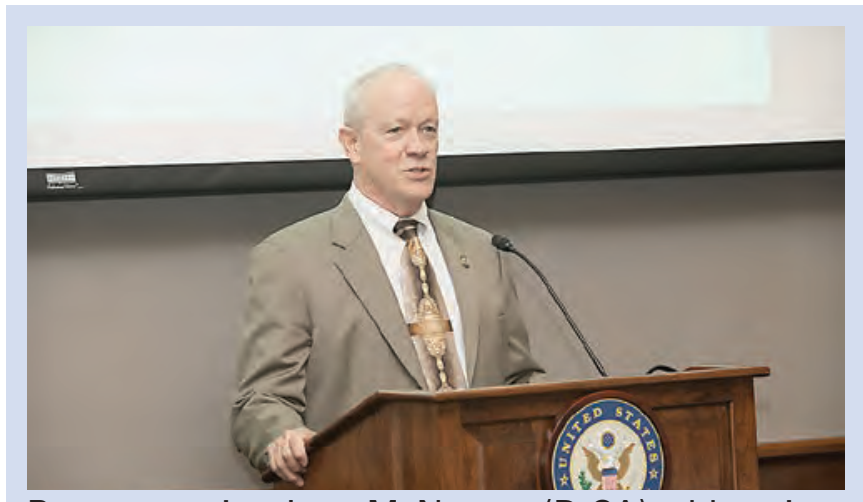

Representative Jerry McNerney (D-CA) addressing the room

Votes on the House floor prevented Nancy Pelosi and other legislators from attending the briefing, but Jerry McNerney (D-CA), the only math PhD in Congress, caught the beginning of the event and offered some remarks. An excerpt:

"I think as mathematicians you want to stick to reality, to facts, and to truth, and nowadays that's even more important than ever. We need people that are really evidence-based, people that want to get things done for the good of the country and not so much for the political gain that we seem to think is good. And so I encourage you to get involved in the process a little bit. There is a lot at stake now in Washington, and it's good to see people engaged at some level."

\section{References}

[1] Demaine Erik D, Langerman S, and O'Rourke J, Geometric Restrictions on Producible Polygonal Protein Chains, Algorithmica, volume 44, number 2, February 2006, pages 167-181. Special issue of selected papers from the 14th Annual International Symposium on Algorithms and Computation, 2003. MR2194474

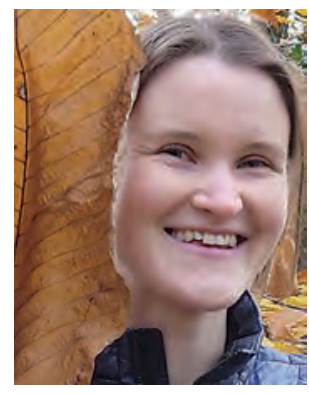

Sophia D. Merow

\section{Credits}

Author photo is by Craig Merow.

All other article photos are by Scavone Photography. 


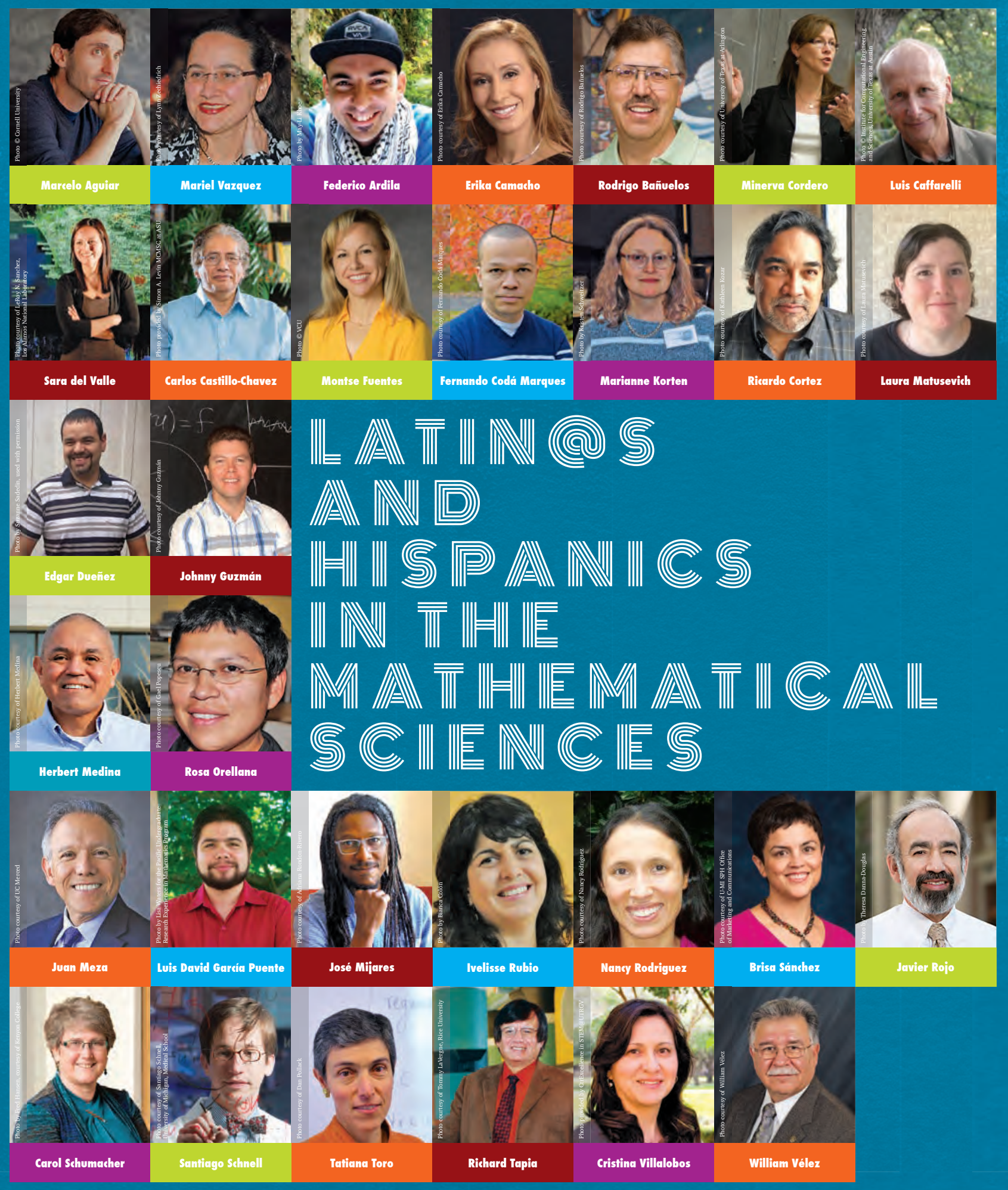

Explore the research, inspiring lives and mentoring contributions of these Latin@s and Hispanics in different areas of the mathematical sciences.

Lathisms.org 


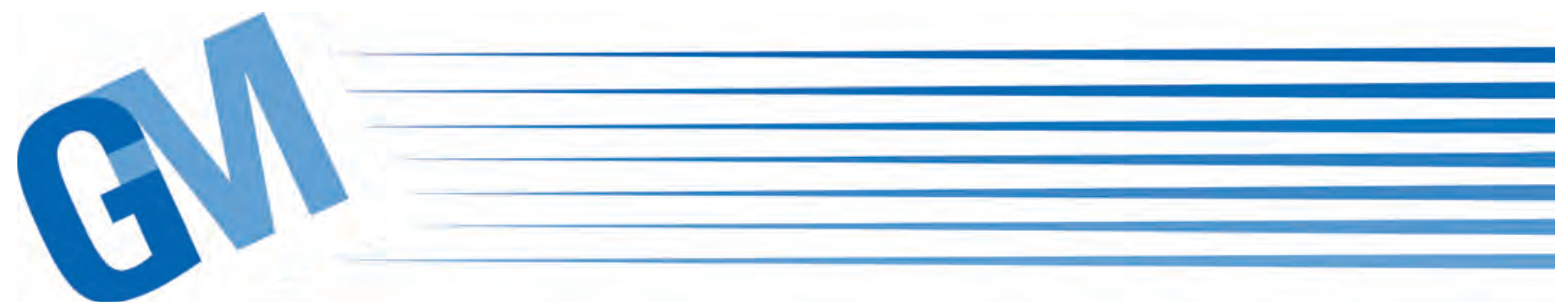

National laboratories are interdisciplinary institutions that tackle important problems from energy and infrastructure to national security. In addition to current discoveries in these technical domains, national labs have a long history of highly impactful breakthroughs. In this column we learn about a current application of differential equations at Pacific Northwest National Laboratory to understand correlated noise that occurs in power systems. We also hear about how the Monte Carlo method for solving complex problems in a variety of disciplines, introduced by Los Alamos National Laboratory, is still being used and advanced there today.

\section{Stochastic Resonance When Uncertainty Meets Dynamics}

\section{David Barajas-Solano and Zhenyu Huang}

Due to the increase in penetration of renewable energy sources and intelligent load devices, power systems are subject to significantly larger uncertainties in power generation and consumption stemming from fluctuating, difficult-to-predict weather conditions and modern demand profiles. Such power fluctuations are characterized by multiple, non-trivial correlation timescales ranging from seconds to minutes. Therefore, the question arises in terms

David Barajas-Solano is a mathematician in the Computational Mathematics Group within the Physical and Computational Sciences Directorate at Pacific Northwest National Laboratory. His email address is David. Barajas-Solano@pnn1.gov.

Zhenyu Huang is an electrical engineer in the Electricity Infrastructure and Buildings Group within the Energy and Environment Directorate at Pacific Northwest National Laboratory. His email address is Zhenyu. Huang@pnn 1.gov.

Communicated by Notices Associate Editor Emilie Purvine.

For permission to reprint this article, please contact: reprint - permission@ams.org.

DOI: http://dx.doi.org/10.1090/noti1766 of how such fluctuations interact with electromechanical timescales such as inter-area low-frequency oscillation periods that are in the order of seconds [1], and what the effect is of such interactions on the dynamics and stability of power systems. This becomes a fundamental challenge in assessing and optimizing power system operations under uncertainties to ensure reliability, resilience, and security of the critical electric infrastructure. At Pacific Northwest National Laboratory, we are tackling this challenge by combining our unique power system domain expertise and computational mathematics capabilities.

The so-called Probability Density Function (PDF) method is a tool for analyzing the one-point PDF, i.e., the probability of being at a certain configuration at a certain time, of the state of general dynamical systems driven by autocorrelated noises [2]. We have successfully adapted and applied the PDF method to the stochastic differential equations governing power system dynamics. Such PDFbased analysis reveals that power generation fluctuations with a specific correlation time magnify the amplitude of the fluctuations around certain operating conditions [3]. Furthermore, this analysis shows that the resonance correlation time is related to the electromechanical timescales of the power system [4]. This "stochastic resonance" phenomenon illustrates the importance of quantifying and accounting for the effect of the correlation time of stochastic drivers on dynamical systems. This is a foundational discovery for large-scale power system uncertainty assessment. It offers a whole new insight in how to assess and mitigate the impact of uncertainties on power systems and potentially help to answer fundamental questions such as how much renewable energy a power system can 
accommodate without losing dynamic stability and how to improve power system controls to enable more renewable energy to be integrated.

\section{The Monte Carlo Method: From Then to Now}

\section{J. E. Gubernatis}

The work of Ulam and von Neumann shortly after World War II on radiation transport and the additional work of Metropolis et al. [5] a few years later on the melting of solids laid the foundations for most of the Monte Carlo methods used today and give the Los Alamos National Laboratory the honor of being the birthplace of the Monte Carlo method. The Monte Carlo method refers to the use of stochastic procedures to simulate or otherwise solve problems that are too complex to solve deterministically. In the first publication to use the rubric "the Monte Carlo method," Metropolis and Ulam [6] in fact saw the approach as something scientists would from time to time use to overcome what we today call the "curse of dimensionality," the often exponential increase in difficulty in analyzing problems in high dimensional spaces.

At Los Alamos, the original work of Ulam and von Neumann grew into the development and maintenance of the Monte Carlo N-Particle (MCNP) simulation code, the international "gold standard" for simulation radiation transport not only for national nuclear security programs but also for nuclear reactor and waste storage applications. The seminal work of Metropolis et al. proved to be exceptionally application neutral so its importance expanded at Los Alamos and elsewhere to hosts of complex problems across all the physical, mathematical, medical, information, financial, computer, and social sciences. Of specific current interest is the application of the Monte Carlo method to large-scale optimization problems in machine learning. A relatively recent growth area is the extension of the method to problems obeying the laws of quantum mechanics. In fact, Metropolis and Ulam credit the Nobel laureate E. Fermi for first noting this possibility.

Today, at Los Alamos, quantum Monte Carlo is being used as a research tool in quantum chemistry, nuclear physics, high-energy physics, quantum field theory, and condensed matter physics.

\section{References}

[1] Zhenyu Huang, Ning Zhou, Francis Tuffner, Yousu Chen, Daniel Trudnowski, William Mittelstadt, John Hauer, Jeffery Dagle. Improving Small Signal Stability through Operating Point Adjustment, Proceedings of the IEEE Power and Energy Society General Meeting 2010, Minneapolis, MN, July 25-29, 2010.

[2] David A Barajas-Solano, Alexandre M Tartakovsky. Probabilistic density function method for nonlinear dynamical systems driven by colored noise, Physics Review E, 93(5), 052121, 2016. MR3709429

[3] W Steven Rosenthal, Alexandre M Tartakovsky, Zhenyu Huang. Ensemble Kalman Filter for Dynamic State Estimation of Power Grids Stochastically Driven by Time-correlated Mechanical Input Power, IEEE Transactions on Power Systems, 33(4), 3701-3710, 2018.

[4] Peng Wang, Alexandre Tartakovsky, Zhenyu Huang, Barry Smith, Shri Abhyankar. Probabilistic Density Function Method for Stochastic ODEs of Power Systems with Uncertain Power Input, SIAM/ASA Journal on Uncertainty Quantification, 3(1), 873-896, 2015. MR3400032

[5] N Metropolis, A W Rosenbluth, M N Rosenbluth, A H Teller, E Teller. Equations of state by fast computing machines, Journal of Chemical Physics, (21): 1087-1092, 1953.

[6] N Metropolis, S Ulam. The Monte Carlo Method, Journal of the American Statistical Association, (44): 335-341, 1949. MR0031341

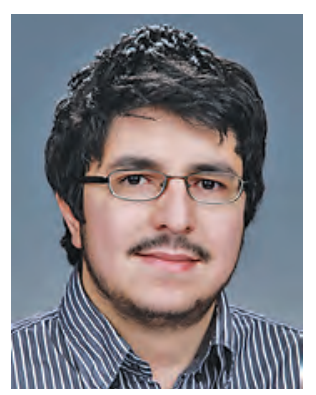

David Barajas-Solano

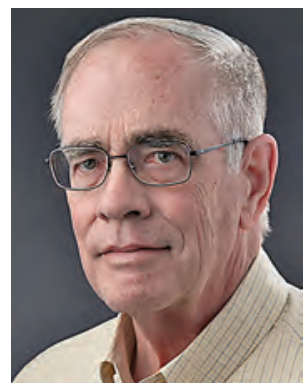

J. E. Gubernatis

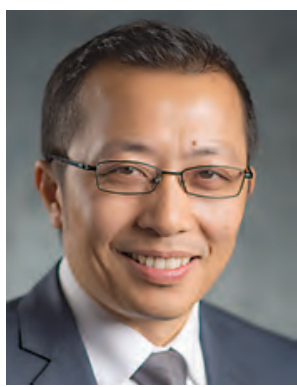

Zhenyu Huang

\section{Credits}

Photos of Barajas-Solano and Huang are courtesy of Pacific Northwest National Laboratory.

Photo of Gubernatis is courtesy of Michael Pierce/Los Alamos National Laboratory. 


\section{Science for Policy: My Year on Capitol Hill}

\section{Margaret D. Callahan}

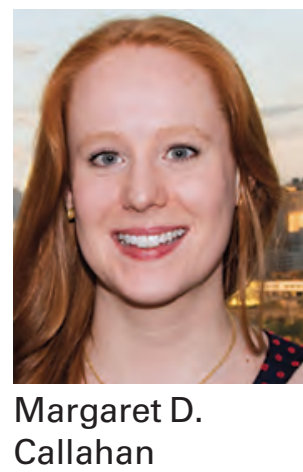

This past year, I had the incredible privilege of serving as the AMS Congressional Fellow. I was one of thirty-six $\mathrm{PhD}$ scientists with a wide range of scientific backgrounds serving in Congress-in personal offices and on committee staff, in both chambers, and on both sides of the aisle. Although the American Association for the Advancement of Science (AAAS) organizes the Congressional Science and Engineering Fellowship program, nearly all of the Fellows in my cohort were sponsored by scientific associations like AMS. The Congressional Fellows joined over 200 Executive Branch Fellows and a single Judicial Branch Fellow to form the 2017-2018 Fellowship class. The goal of the Fellowship is to bring scientific perspective and technical expertise to the legislative process while affording scientists a voice in the conversations that shape our nation's public policy. Given that less than three percent of Congress has a background in science or engineering and the rapidly increasing rate at which our lawmakers are forced to make decisions within highly technical and scientific frameworks, it is more important than ever that scientists are part of the legislative process.

To varying degrees, Congressional Fellows engage in both "science for policy" and "policy for science." Science for policy can be viewed as the use of scientific knowledge or processes to assist in or improve decision-making, whereas policy for science refers to how the federal government funds or structures science research. During my Fellowship year, which I spent working in the office of Senator Amy Klobuchar of Minnesota on issues pertaining to education and workforce development, I primarily

Margaret D. Callahan works as a foreign affairs officer with the US State Department.

For permission to reprint this article, please contact: reprint -permission@ams.org.

DOI: http://dx.doi.org/10.1090/noti1784 engaged in science for policy. I had the opportunity to become knowledgeable about a wide range of timely issues, including protections for student loan borrowers, access to apprenticeships and career training programs, innovations in STEM education, the rights of labor unions, child care shortages, and so much more.

Over the course of the year, I participated in many different aspects of the legislative process, and no two days were alike. I attended hearings and briefings on topics of interest and relevance to the senator and drafted meeting memos, legislative background materials, letters, and talking points. I researched and analyzed bills and letters in order to help advise the senator on whether or not she should lend her support (typically in the form of a cosponsorship or signature, respectively). I worked with Minnesota experts and stakeholders, colleagues on both sides of the aisle, and legislative counsel to draft legislation, and strategized with senior staff on how to get new bills introduced and passed. I took meetings with constituent, advocacy, and industry groups, learning about important issues affecting Minnesotans and building helpful connections with people on the ground.

While I did not have the opportunity to leverage my specific training as an applied mathematician to further my boss' policy priorities, I did use the skills I obtained over the course of my doctoral studies and in doing mathematical research. My research skills and analytical mindset were useful in the vetting of new legislation that other senators had asked Senator Klobuchar to cosponsor. Problem solving, critical thinking, and creativity lent themselves to the development of new ideas-legislative and otherwise-to address problems facing people in Minnesota and across the country. In researching and drafting new legislation, I was able to bring my own sense of scientific rigor to the process, whether by ensuring that data supports the foundation of a particular bill or by demanding clarity and logical flow in the language. A Congressional Fellow has 


\section{COMMUNICATION}

the opportunity to be more than a voice for science and for evidence-based policy-making in their host office-they can bring elements of the scientific process into their work every day.

In addition to the personal and professional satisfaction of contributing in a tangible way to the formulation of policy solutions to the problems facing our nation, $\mathrm{PhD}$ scientists have a great deal to gain from an experience working on Capitol Hill. Over the course of my Fellowship year, I established a broad and diverse professional network consisting of fellow AAAS Fellows, colleagues from my Senate office, and science policy professionals across the DC area. I developed a comprehensive understanding of the policy-making process and sense of how scientists fit into that process. I gained the ability to multitask in an intense and fast-paced work environment and further strengthened my skills in communication to a wide variety of audiences. The Fellowship gave me the opportunity to contribute my skills and experiences as a mathematician-quite unique to Capitol Hill-to the policy-making process, and it helped me see how much the work and perspectives of scientists are valued by lawmakers.

I had a truly fantastic experience as the AMS Congressional Fellow. Before starting the Fellowship, in part because of the current political climate, many people expressed skepticism about the experience I was likely to have and what I would be able to realistically contribute. However, through this experience-in particular, in working with some of the smartest, most dedicated and hardworking people in my Senate office-I have become, if anything, less cynical about the work that is done on Capitol Hill. Most people I have met here are motivated by an honest desire to serve their country and to improve peoples' lives. The experience has been humbling and inspiring and I have learned more than I ever dared hope. I am immensely grateful to AMS for sponsoring this Fellowship and for affording me the honor of serving as its Fellow.

The AMS sponsors one fellowship placement in Congress each year. Application deadline for the AMS Congressional Fellowship is February 15, 2019. Learn more and apply at http://bit. 7y/2LaVL1B.

There will be an information session about this Fellowship at the Joint Mathematics Meetings in Baltimore, MD on Friday, January 18, 2019, at 4:30 pm in Room 316 of the Baltimore Convention Center. Come and find out more about this unique opportunity!

\section{Credits}

Author headshot is courtesy of the author. 


\section{Community Updates}

\section{Trjitzinsky Awards}

The AMS has made awards to eight undergraduate students through the Waldemar J. Trjitzinsky Memorial Fund. The fund is made possible by a bequest from the estate of Waldemar J., Barbara G., and Juliette Trjitzinsky. The will of Barbara Trjitzinsky stipulates that the income from the bequest should be used to establish a fund in honor of the memory of her husband to assist needy students in mathematics.

For the 2018 awards, the AMS chose seven geographically distributed schools to receive one-time awards of US $\$ 3,000$ each. The mathematics departments at those schools then chose students to receive the funds to assist them in pursuit of careers in mathematics. The schools are selected in a random drawing from the pool of AMS institutional members.

Waldemar J. Trjitzinsky was born in Russia in 1901 and received his doctorate from the University of California Berkeley in 1926. He taught at a number of institutions before taking a position at the University of Illinois, Urbana-Champaign, where he remained for the rest of his professional life. He showed particular concern for students of mathematics and in some cases made personal efforts to ensure that financial considerations would not hinder their studies. Trjitzinsky was the author of about sixty mathematics papers, primarily on quasi-analytic functions and partial differential equations. A member of the AMS for forty-six years, he died in 1973.

Following are the names of the selected schools for 2018, the names of the students receiving the awards, and brief biographical sketches of the students.

Trinity College: Coawardees are AASHWIN BASNET and Daniel Melesse. Aashwin Basnet, born in a small village in Kathmandu, Nepal, is a senior at Trinity College with a double major in mathematics and theoretical physics and a minor in religious studies. His love of mathematics has been especially inspired by calculus, linear algebra, and the applicability of mathematics to quantum mechanics. Following a year of study at the University of Edinburgh, Aashwin is now working on a project to make a handheld, efficient, and inexpensive coincidence counting module (CCM) device to be used for experiments such as quantum entanglement and radioactive decay. In his senior year, he will be serving a third term as a calculus teaching assistant while studying Lie algebras and PDEs. He will also be applying to graduate programs with an aim to pursue a doctoral degree in theoretical particle physics. Aashwin is one of Trinity's leading citizens with his constantly cheerful presence as a regular TA and is a role model for STEM double majors.

Daniel Melesse, from Bahir Dar, Ethiopia, is a member of the class of 2020. He is double majoring in mathematics and electrical engineering and minoring in models and data. In his freshman year, Daniel conducted research in the math department on synchronization in nonlinear harmonic oscillators. Since then, he has been working on a research project titled "Data Driven Approach for Automatic Gaze Tracking" in Trinity's robotics lab. This collaborative project involves the use of advanced mathematics to understand and apply different machine-learning algorithms and has resulted in the acceptance of a research paper at the Fourteenth IEEE International Conference in Signal Processing in China. Outside of academics, Daniel is a tutor at the Aetna Quantitative Center at Trinity on topics ranging from college algebra to differential equations. He is a Deans' Scholar for the class of 2020, which recognizes first-year students with the twenty-five highest GPAs at the end of the first year, and he was awarded a Phi Gamma Delta Prize in Mathematics for First-Year Students. His future plans include graduate school to pursue a $\mathrm{PhD}$ in signal processing/machine learning.

University of Kentucky: Claire BARRERA grew up in the suburbs of Chicago, where she lived with her parents and older brother. In high school she was a member of the Cares Club, where the focus was on helping underprivileged children in the community by providing materials to aid in their academic success. After high school Claire elected to attend the University of Kentucky to pursue a bachelor of science degree in mathematics. She chose mathematics because she enjoys problem solving and it was always a subject she excelled in. In addition, she is a member of the Center for Academic Resource and Enrichment Services (CARES) program, where they help to provide academic support to students who are underrepresented at the University of Kentucky. She began tutoring many friends in a variety of math classes and, given the good results of her tutoring, she decided to work on a regular basis as an undergraduate assistant in the Mathskeller, the learning 
center maintained by the Department of Mathematics at Kentucky. Claire is also minoring in economics and is interested in combining her two passions by pursuing a career in actuarial science or in the business/finance field.

Hobart and William Smith Colleges: HADLEY DEBRINE is a double mathematics and art major in the class of 2020 at Hobart and William Smith Colleges. She has a deep appreciation for mathematics and loves applying mathematical concepts to all aspects of her life, such as in her art classes. Although Hadley has been regularly challenged by nonacademic obstacles that can distract from scholarly activities, she has remained dedicated to exploring mathematics. Her professors have been impressed with her ability to understand the subtleties of proof and believe she has a knack for unpacking abstract ideas.

California State University, Los Angeles: LuIs Ruiz earned his GED at the age of twenty after dropping out of the South Los Angeles school system in ninth grade. Shortly after, he enrolled in a local community college, where he began his mathematical journey with an arithmetic class. His instructor saw something special in Luis and encouraged him to pursue a degree in mathematics. No one he knew had ever attended college, let alone graduated. He felt he was wasting his time going to school. That view quickly changed as he continued through the community college, earning the Walter $\mathrm{O}^{\prime}$ Connell Endowment in 2016 and being selected by the National Science Foundation as a STEM Scholar. He was recruited to help student retention at the college's math tutoring center and was hired two consecutive years as a supplemental instruction mentor (SI) for the math department. Currently, Luis is a senior at California State University, Los Angeles, pursuing a bachelor of science in mathematics. He works as a high school math tutor. Luis plans to pursue a $\mathrm{PhD}$ in pure mathematics. His dream is to become a mathematics professor and instill his passion for mathematics in others.

University of Dayton: MARY GHILONI is a junior who is pursuing degrees in mathematics and in adolescent to young adult education at the University of Dayton. She is a member of the Ohio Zeta chapter of Pi Mu Epsilon and the secretary of the Math Club. In the summer of 2018, she received a Dean's Summer Research Scholarship. She researched the curricula of Finland, Singapore, and the United States and looked in depth into how these countries develop the concepts of addition and subtraction through their textbooks. Mary is passionate about both education and mathematics and hopes to become not only a teacher but also a mentor to her students.

Western Washington University: STACY STONES is a junior math major who is also interested in computer science and has completed a minor in French. She has been fascinated with mathematics all her life and enjoys solving challenging problems. A first-generation college student who worked for a few years prior to starting college, Stacy at first found it difficult to navigate college and to understand what was necessary for success. She feels that hard work, genuine interest, and the support of faculty and classmates are all essential to succeed. She began her mathematical studies at Western Washington with precalculus, was interviewed and selected for the honors calculus track the following year, and has continued to grow in her upper-division coursework. While she does not know exactly what she would like to do after graduation, she is excited about exploring career opportunities and is considering graduate study in the field. No matter where life takes her, she knows that math will be an important part of it.

South Dakota State University: YIRONG WANG was born and raised in Liaoning, China, and moved to the United States when she was seventeen. Throughout high school, she worked hard on studying English and discovered her strong interest in mathematics. She graduated from Brookings High School in South Dakota with GPA of 4.0 in 2017 and decided to study mathematics at South Dakota State University. The study of math is important to Yirong because she feels mathematicians have an opportunity to make a lasting contribution to society by helping to solve problems in many different fields, such as medicine, the economy, health systems, biology, and finance, among others. Her goal is to become a data scientist. Yirong worked at the Bioinformatics and Mathematical Biosciences Lab of South Dakota State University this past summer. This lab uses efficient data mining and modeling skills to solve problems and develop bioinformatics tools. She feels she learned a lot from this experience. Yirong is grateful to be a recipient of the Waldemar J. Trjitzinsky Scholarship, as it will help her to support her college life and to pursue her academic goals. 


\section{NEWS}

\section{Erdős Memorial Lecture}

The Erdős Memorial Lecture is an annual invited address named for the prolific mathematician Paul Erdős (19131996). The lectures are supported by a fund created by Andrew Beal, a Dallas banker and mathematics enthusiast. The Beal Prize Fund is being held by the AMS until it is awarded for a correct solution to the Beal Conjecture (see www. math. unt. edu/ mauldin/bea1.htm7). At Mr. Beal's request, the interest from the fund is used to support the Erdős Memorial Lecture.

LaUREN K. Williams of the University of California Berkeley will present the 2019 Erdős Memorial Lecture during the Fall 2019 Central Sectional Meeting at the University of Wisconsin-Madison, September 14-15, 2019. See https://www.ams.org/meetings/lectures/ meet-erdos-1ect.

-AMS announcement

\section{From the AMS Public}

\section{Awareness Office}

AMS Blogs, written by mathematicians, cover a range of topics of interest to mathematicians at all stages of their careers. Recent posts include "Math Games That Make You Think," "Good News! Great Reports Now Available to All!" (on the release of Congressional Research Service reports), "Requesting Letters of Recommendation," "Converging on a Solution: A Playwright's Path," "Illuminating Skills Learned from Teaching," "\#thestruggleisREAL: Reflection in a Real Analysis Class," and "Giving Bad Colloquia." Browse the collection of AMS Blogs at https://blogs. ams . org, share feedback in the comments, and subscribe to receive email notifications of new posts.

-Annette Emerson and Mike Breen AMS Public Awareness Officers paoffice@ams.org

\section{Introducing the Next Generation Fund}

The Next Generation Fund is a new endowment at the AMS that exclusively supports programs for doctoral and postdoctoral scholars. It will assist rising mathematicians each year at modest but impactful levels, with funding for travel grants, collaboration support, mentoring, and more!

To learn more or to make a gift, go to www . ams . org/ giving. 


\section{Mathematics People}

\section{Liu and Thorne Awarded SASTRA Ramanujan Prize}

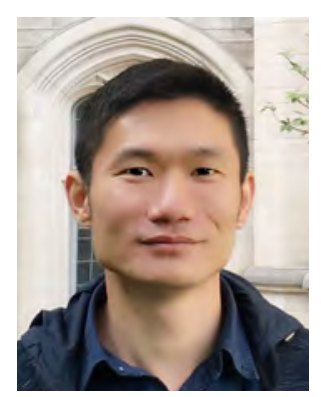

Yifeng Liu

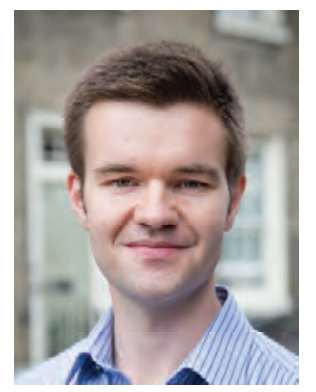

Jack Thorne

YIFENG LIU of Yale University and JACK THORNE of Cambridge University have been named the recipients of the 2018 SASTRA Ramanujan Prize. The prize citation for Liu reads as follows: "Yifeng Liu is awarded the 2018 SASTRA Ramanujan Prize for his many spectacular contributions to arithmetic geometry and number theory. The prize recognizes his marvelous $2012 \mathrm{PhD}$ thesis at Columbia University entitled 'Arithmetic Inner Product Formula for Unitary Groups,' which includes his fundamental work on arithmetic theta lifting and $L$-derivatives that appeared in two substantial papers in Algebra and Number Theory in 2011. The prize also recognizes his subsequent three papers pertaining to Bessel and Fourier-Jacobi models that appeared in Journal of Functional Analysis in 2013 (coauthored with Binyong Sun), Manuscripta Mathematica in 2014, and Crelle's Journal [Journal for Pure and Applied Mathematics] in 2016, in which he made major progress on the Gan-Gross-Prasad conjectures in the representation theory of classical groups. The prize also notes that in his 2018 paper in the Duke Mathematics Journal (coauthored with Shouwu Zhang and Wei Zhang), important $p$-adic versions of theorems of Waldspurger and Gross-Zagier are established, generalizing earlier fundamental work of Bertolini, Darmon, and Prasanna. In the '80s, Gross-Zagier and Kolyvagin proved some amazing theorems which implied the celebrated Birch-Swinnerton-Dyer conjecture for certain elliptic curves. The prize recognizes that in his 2016 paper in Inventiones Mathematicae, as well as in a paper to appear in the Journal of the EMS, and in subsequent joint work, Liu has extended Kolyvagin type results to higher ranks in the general framework of the Beilinson-Bloch-Kato conjecture. In addition, the prize notes that he has made inroads also into non-Archimedean geometry, as evidenced by his 2011 paper in the Journal of Differential Geometry, where he has established a non-Archimedean analogue of the famous Calabi Conjecture for abelian varities over $p$-adic fields with complete degeneration. Thus he has established himself as a leading and influential figure in arithmetic geometry, automorphic representations and number theory, and his work is expected to have major impact in these areas in the future."

The citation for Thorne reads: "Jack Thorne is awarded the 2018 SASTRA Ramanujan Prize for his far-reaching contributions to number theory, representation theory and arithmetic geometry, especially to the modularity of Galois representations and arithmetic invariant theory. The prize recognizes his outstanding $2012 \mathrm{PhD}$ thesis at Harvard University entitled 'The Arithmetic of Simple Singularities'; one outcome of this was his 2013 paper on arithmetic invariant theory that appeared in Algebra and Number Theory, which leads to new bounds on the sizes of certain Selmer groups, and on the number of rational and integral points on various classes of algebraic curves. The prize notes that concerning modularity of Galois representations, Thorne has been a central force in eliminating restrictions on the Taylor-Wiles method, as evidenced in his three seminal papers with Laurent Clozel on level raising and symmetric power functoriality in Compositio Mathematica in 2014, the Annals of Mathematics in 2015, and the Duke Journal in 2017. Of note in his joint work with Clozel is Thorne's discovery and use of a surprising automorphy lifting theorem that was established in his 2015 paper in the Journal of the American Mathematical Society. The works of Thorne and of Clozel-Thorne are expected to greatly extend the scope of the Taylor-Wiles method. The prize recognizes that Thorne's 2015 joint work with Chandrashekhar Khare on potential automorphy and the Leopoldt conjecture, to appear in the American Journal of Mathematics, has led to a proof of a potential version of the Shimura-Taniyama conjecture for elliptic curves over imaginary quadratic fields, in a major project involving several researchers, including Thorne. Finally, the prize notes that Thorne's recent paper to appear in the European Journal of Mathematics establishing that all elliptic curves over $Q_{\infty}$ are modular is another major breakthrough. With his outstanding contributions to 


\section{NEWS}

two distinct areas of number theory/arithmetic geometry, and his ability to overcome technical obstacles, Thorne has become one his generation's leaders in the field of algebraic number theory."

The prize committee for the 2018 SASTRA Ramanujan

Prize consisted of:

- Krishnaswami Alladi, Chair, University of Florida

- David Bressoud, Macalester College

- Gerhard Frey, University of Essen

- Andrew Granville, University of Montreal; University College, London

- Alex Lubotzky, Hebrew University

- Philippe Michel, Ecole Polytechnique, Lausanne

- Gisbert Wustholz, ETH Zurich

- Previous winners of the SASTRA Ramanujan Prize are:

- Manjul Bhargava and Kannan Soundararajan (two full prizes), 2005

- Terence Tao, 2006

- Ben Green, 2007

- Akshay Venkatesh, 2008

- Kathrin Bringmann, 2009

- Wei Zhang, 2010

- Roman Holowinsky, 2011

- Zhiwei Yun, 2012

- Peter Scholze, 2013

- James Maynard, 2014

- Jacob Tsimerman, 2015

- Kaisa Matomaki and Maksym Radziwill (shared), 2016

- Maryna Viazovska, 2017

—Krishnaswami Alladi, University of Florida

\section{Sly Awarded MacArthur Fellowship}

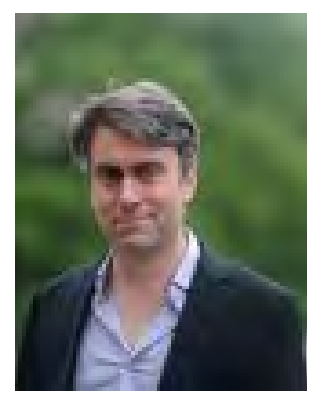

Allan Sly
ALLAN SLY of Princeton University has been awarded a MacArthur Fellowship, popularly known as a "genius grant," for 2018.

According to the prize citation, "Allan Sly is a mathematician and probability theorist resolving long-standing open problems in statistical physics and theoretical computer science.

"Sly's accomplishments include important findings pertaining to the threshold for recovering clusters in the sparse stochastic block model; pathbreaking work on cutoff in Markov chains; and the discovery of a key to constructing embeddings of random sequences into random sequences. He has also determined a proof of the satisfiability conjecture for large $k$, linking a

fundamental problem in theoretical computer science to one in statistical physics. To solve these problems that have confounded many strong mathematicians before him, Sly develops novel tools with broad applicability. For example, he has introduced an innovative strategy called "information percolation" to analyze the cutoff phenomenon in Ising-Glauber models (the existence or not of a sharp transition within a short time window from an unmixed state to the mixed equilibrium state), new graphical methods for the proof of the satisfiability conjecture, and a new geometric approach to the slow bond problem.

"Through conceptual breakthroughs in methodology, Sly is making fundamental progress on important and difficult problems that are of central interest to mathematics and have important applications in many other fields."

Allan Sly received his PhD in statistics from the University of California at Berkeley in 2009 under the direction of Elchanan Mossel. He was a postdoctoral fellow at Microsoft Research (2009-2011) and a member of the Department of Statistics at the University of California at Berkeley (2011-2016) before joining the faculty at Princeton University, where he is currently a professor in the Department of Mathematics.

_From a MacArthur Foundation announcement

\section{Logunov Awarded 2018 Salem Prize}

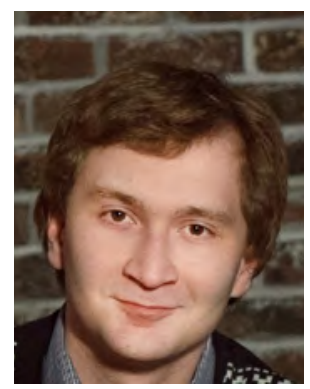

Alexander Logunov
AlEXANDER LOGUNOV of the Institute for Advanced Study and Princeton University has been awarded the 2018 Salem Prize for his work on the conjectures of Yau and Nadirashvili on the volumes of the zero sets of Laplacian eigenfunctions. His work centers on harmonic analysis, potential theory, and geometric analysis. He received his PhD from St. Petersburg State University in 2015 under the supervision of Viktor Havin. He spent two years as a postdoctoral fellow at Tel Aviv University before moving to Princeton. In 2017 he received the Clay Research Award jointly with Eugenia Malinnikova for their introduction of novel geometric-combinatorial methods for the study of elliptic eigenvalue problems, and he has been appointed as a Clay Research Fellow for a two-year term beginning in July 2018.The prize, in memory of Raphael Salem, is awarded yearly to young researchers for outstanding contributions to the field of analysis. 


\section{Håstad Awarded Knuth Prize}

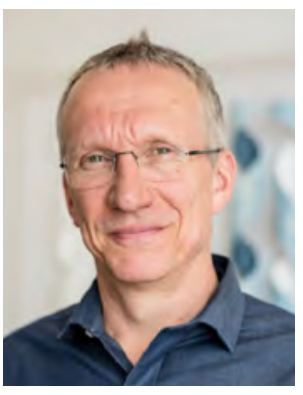

Johan Håstad

JOHAN HÅSTAD of KTH Royal Institute of Technology, Stockholm, Sweden, E. Knuth Prize "for his long and sustained record of milestone breakthroughs at the foundations of computer science, with huge impact on many areas including optimization, cryptography, parallel computing, and complexity theory." The prize is has been awarded the 2018 Donald sponsored jointly by ACM SIGACT and IEEE TCMF. According to the prize citation, his "multiple seminal works have not only resolved longstanding deepest problems central to circuit lower bounds, pseudorandom generation, and approximability, but also introduced transformative techniques that have fundamentally influenced much of the subsequent work in these areas." Håstad received his $\mathrm{PhD}$ from the Massachusetts Institute of Technology in 1986. In his spare time, Håstad enjoys a glass of good wine, picking wild mushrooms, and skating on the frozen lakes of Sweden in the winter.

-From an ACM announcement

\section{AWM Fellows Chosen}

The Executive Committee of the Association for Women in Mathematics (AWM) established the AWM Fellows Program to recognize individuals who have demonstrated a sustained commitment to the support and advancement of women in the mathematical sciences, consistent with the AWM mission: "to encourage women and girls to study and to have active careers in the mathematical sciences, and to promote equal opportunity and the equal treatment of women and girls in the mathematical sciences."

The 2019 class of AWM Fellows are researchers, mentors, and educators who are recognized by their peers and students for their commitment to supporting women in the mathematical sciences.

Following are the names and institutions of the 2019 AWM Fellows.

- Hélène Barcelo, Mathematical Sciences Research Institute

- Lida Kittrell BarRett

- Sun-Yung Alice Chang, Princeton University

- Amy Cohen, Rutgers University

- Ingrid Daubechies, Duke University

- Chandler Davis, University of Toronto

- JaCQueline Dewar, Loyola Marymount University

- Edray Herber Goins, Pomona College

- Judy Green, Marymount University
- PaO-sheng Hsu, Independent

- Ellen E. KiRKMan, Wake Forest University

- Maria M. Klawe, Harvey Mudd College

- Anne M. LeggetT, Loyola University Chicago

- Magnhild Lien, California State University, Northridge

- Maeve Lewis McCarthy, Murray State University

- Dusa McDuff, Barnard College, Columbia University

- Irina Mitrea, Temple University

- Alice Silverberg, University of California Irvine

- Audrey Terras, University of California San Diego

- Marie A. Vitulli, University of Oregon

- Judy LeavitT Walker, University of Nebraska-Lincoln

- LesLey WARD, University of South Australia

- Ulrica Wilson, Morehouse College

_From an AWM announcement

\section{ICIAM Prizes for 2019}

\section{Announced}

The International Council for Industrial and Applied Mathematics (ICIAM) has announced several major prizes to be awarded at its 2019 Congress in Valencia, Spain, in July 2019.

SidDhARTHA Mishra of ETH Zurich receives the Collatz Prize "for his breakthrough contributions that skillfully combine modeling of real-world problems and rigorous mathematical analysis with the development of efficient and accurate numerical schemes and high-performance computing." The prize recognizes scientists under forty-two years of age for outstanding work in industrial and applied mathematics.

GEORGE PAPANICOLAOU of Stanford University was named the recipient of the Lagrange Prize "for his brilliant use of mathematics to solve important problems in science and engineering; in particular, problems involving inhomogeneity, wave propagation, random media, diffusion, scattering, focusing, imaging, and finance." The prize recognizes mathematicians who have made exceptional contributions to applied mathematics throughout their careers.

Claude Bardos of Université Paris Denis Diderot (Paris 7) is recognized with the Maxwell Prize "for his seminal contributions to nonlinear partial differential equations, kinetic theory, and mathematical fluid mechanics." The prize honors a mathematician who has demonstrated originality in applied mathematics.

YvON MADAY of the Sorbonne and the Universite Pierre et Marie Curie has been selected to receive the Pioneer Prize "in recognition of his leading role in the introduction of powerful methods for numerical simulation, such as spectral methods, reduced order modeling, domain decomposition, models and simulation in medical sciences, 


\section{NEWS}

fluid-structure interaction, and ab-initio chemistry." The prize is awarded for pioneering work introducing applied mathematical methods and scientific computing techniques to an industrial problem area or a new scientific field of applications.

Giulia Di NunNo of the University of Oslo receives the Su Buchin Prize "for her long-lasting record of actively and efficiently encouraging top-level mathematical research and education in developing African countries." The prize recognizes outstanding contributions by individuals in the application of mathematics to emerging economies and human development, in particular at the economic and cultural levels in developing countries.

_From an ICIAM announcement

\section{Photo credits}

Photo of Yifeng Liu credit Shiyue Li.

Photo of Jack Thorne, no credit.

Photo of Allan Sly courtesy of John D. and Catherine T. MacArthur Foundation

Photo of Alexander Logunov, no credit.

Photo of Johan Håstad Courtesy of Andreas Bergsten and KTH Royal Institute of Technology

\section{LEARN ABOUT}

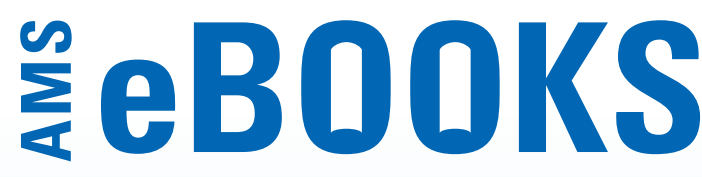

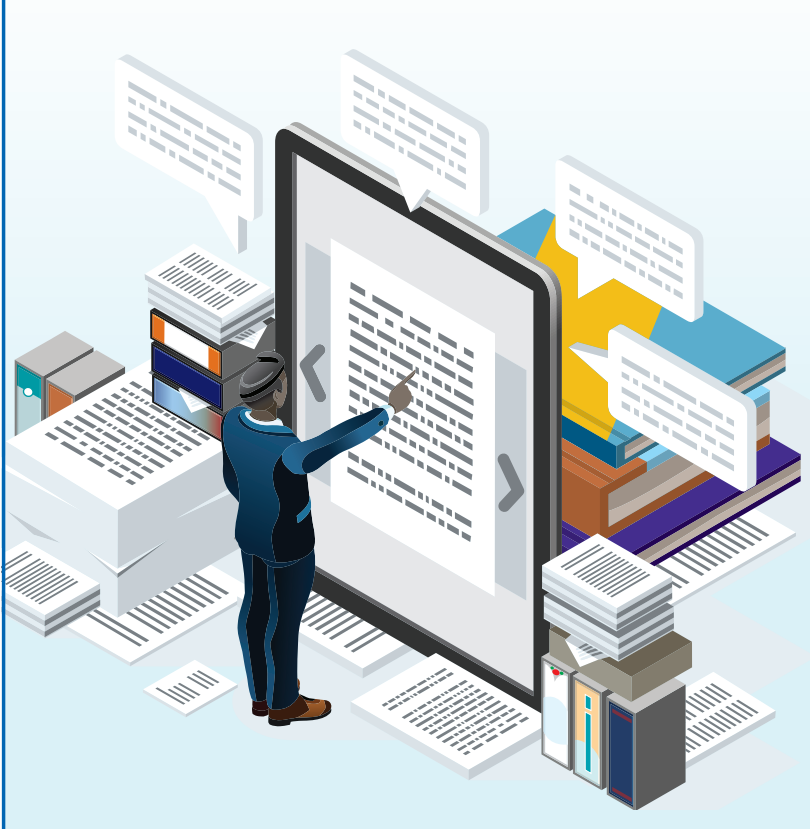

Did you know that most of our titles are now available in eBook form?

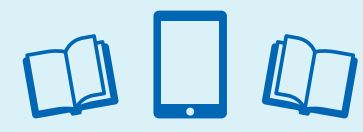

\section{Browse both our print and electronic titles at bookstore.ams.org.}

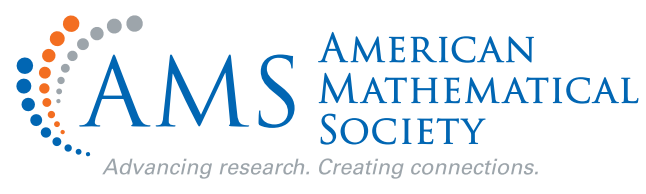




\section{Mathematics Opportunities}

Listings for upcoming mathematics opportunities to appear in Notices may be submitted to noti ces@ams . org.

\section{NSF Algorithms for Threat Detection Program}

The Algorithms for Threat Detection (ATD) program will support research projects to develop the next generation of mathematical and statistical algorithms for analysis of large spatiotemporal datasets with application to quantitative models of human dynamics. The program is a partnership between the Division of Mathematical Sciences at the National Science Foundation and the National Geospatial Intelligence Agency. The deadline for full proposals is February 19,2019 . See https: //www.nsf.gov/funding/ pgm_summ.jsp?pims_id=503427\&org=DMS\&se1_ org=DMS\& from=fund.

-From an NSF announcement

\section{NSF Major Research Instrumentation Program}

The National Science Foundation Major Research Instrumentation program helps institutions of higher education or nonprofit organizations increase access to shared scientific and engineering instruments for research and research training. The deadline is January 22, 2019; see www.nsf.gov/funding/pgm_summ.jsp?pims_ $i d=5260 \&$ org=MPS\&se1_org=MPS\&from=fund

\section{-From an NSF announcement}

\section{Simons Foundation Collaboration Grants for Mathematicians}

The Simons Foundation invites applications for Collaboration Grants for Mathematicians (US $\$ 8,400$ per year for five years). The application deadline is January 31, 2019. See tinyur 1 . com/zvoqm5t.

\section{_From a Simons Foundation announcement}

\section{PIMS Education Prize}

The Pacific Institute for the Mathematical Sciences (PIMS) annually awards a prize to a member of the PIMS community who has made a significant contribution to education in the mathematical sciences. The deadline for nominations is March 15, 2019. See www.pims.math.ca/pimsglance/prizes-awards.

The most up-to-date listing of NSF funding opportunities from the Division of Mathematical Sciences can be found online at: www.nsf.gov/dms and for the Directorate of Education and Human Resources at www.nsf.gov/dir/index.jsp?org=ehr. To receive periodic updates, subscribe to the DMSNEWS listserv by following the directions at www. nsf.gov/mps/dms/about. jsp. 
NEWS

\section{Early Career Opportuntities}

\section{CAIMS/PIMS Early Career Award}

The Canadian Applied and Industrial Mathematics Society (CAIMS) and the Pacific Institute for Mathematical Sciences (PIMS) sponsor the Early Career Award in Applied Mathematics to recognize exceptional research in applied mathematics, interpreted broadly, by a researcher fewer than ten years past earning a $\mathrm{PhD}$. The nominee's research should have been conducted primarily in Canada or in affiliation with a Canadian university. The deadline for nominations is January 31, 2019. See www . pims . math. ca/pims-glance/prizes-awards.

—From a PIMS announcement

\section{CMI-HIMR Summer School in Computational Number Theory}

The Clay Mathematics Institute (CMI) and the Heilbronn Institute for Mathematical Research (HIMR) announce funding for a two-week postgraduate mathematics summer school to be held June 17-28, 2019, at the University of Bristol. Organizers are Jennifer Balakrishnan (Boston University) and Tim Dokchister (University of Bristol). The deadline for applications is March 1, 2019. See www. claymath.org/events/cmi-himrsummer-school-computational-number-theory.

- From a CMI annoucement

\section{Visit us at B00TH 413}

\section{Cambridge University Press at JMM 2019}

\section{Special show discounts | Spin-the-wheel for prizes}
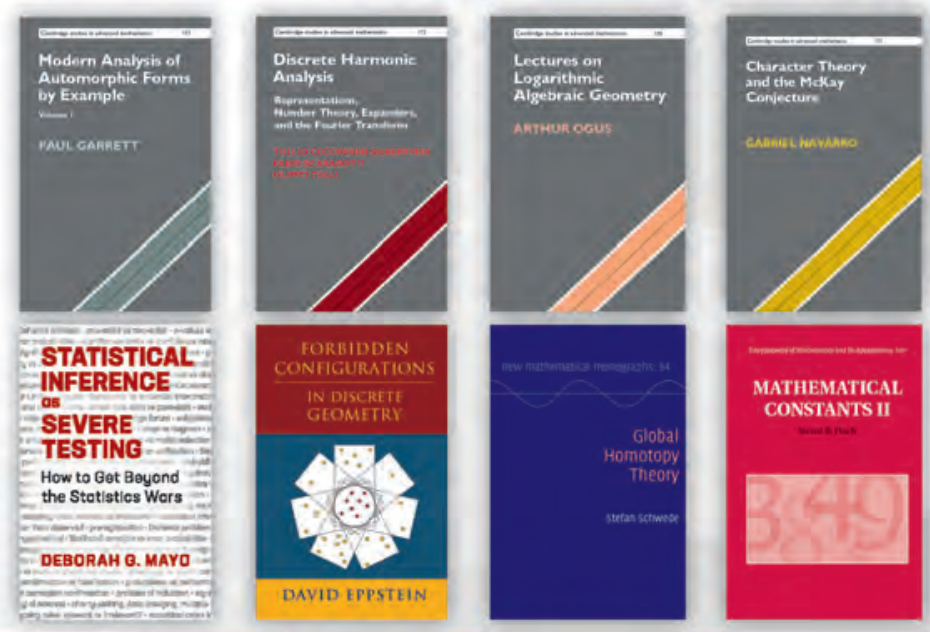

Browse the titles on display online at www.cambridge.org/JMM2019 


\section{Classified Advertising Employment Opportunities}

\section{MISSOURI}

\section{University of Central Missouri School of Computer Science and Mathematics Tenure-Track Position in Actuarial Science \#998534}

The School of Computer Science and Mathematics at the University of Central Missouri (UCM) invites applications for a tenure track assistant professor position in Actuarial Science to begin in August 2019.

The Position: The primary responsibilities of the position are to teach undergraduate and graduate courses in actuarial science, mathematics, and statistics; participate in research and other scholarly activities; engage in student/ faculty collaboration; advise students; and guide students in their professional development. Other responsibilities include service to the department, the university, and the profession. The typical teaching load is 12 credit hours per semester. A reduced teaching load for research and other professional responsibilities is possible.

Qualifications: Applicants must have a PhD degree in actuarial science, mathematics, or statistics by August 2019. Successful candidates should be able to teach a variety of undergraduate and master's level courses. Preference will be given to candidates who are in the process of, or interested in, achieving an Associate or Fellow designation in the Society of Actuaries and can contribute to UCM earning the Centers of Actuarial Excellence designation. The Associate or Fellow designation in the Society of Actuaries must be obtained before promotion and tenure consideration. A strong commitment to excellence in teaching, research, and continued professional growth is essential.

The Application Process: To apply online, go to https: //jobs . ucmo . edu. Apply to position \#998534.

\section{TENNESSEE}

\section{University of Memphis \\ Department of Mathematical Sciences}

The Department of Mathematical Sciences at the University of Memphis is recruiting for a tenure- track Assistant Professor in Statistics to begin in August 2019. Qualifications include a PhD in Statistics Biostatistics or closely related fields, with research interests in Data Science and Bayesian Inference. Details are available at www . memphis . edu/msci/news /positions. php. Application should be completed on-line at https://workforum.memphis.edu /postings/. Review begins on January 2019. Email: eogeorge@memphis.edu in the Fall Semester and $1 i$ hdeng@memphis . edu in the Spring semester. for further questions. EOE. 


\section{CANADA \\ University of Waterloo \\ Executive Director, Institute for Quantum Computing (IOC)}

The Institute for Quantum Computing (IQC) seeks to appoint a new Executive Director. This is a unique opportunity to shape the global future of quantum information science and engineering. Quantum information is an established strategic priority of both the University of Waterloo and the government of Canada. The Executive Director of IQC plays a critical role in the leadership of the Institute by setting its overall strategic direction, cultivating key external relationships, and ensuring that it delivers against its mandate. The Executive Director of IQC reports to the Vice President, University Research.

IQC is a multidisciplinary research center at the University of Waterloo, dedicated to the development and advancement of quantum information science and technology. Established in 2002, it spans the Faculties of Mathematics, Science, and Engineering, and today has an annual budget of approximately $\$ 25$ million and includes 30 faculty members and 40 postdoctoral fellows. IQC is exceptionally well outfitted for advancing quantum science and as a welcoming meeting place for researchers. It has 58,000 square feet of laboratory space in three facilities, including purpose- built laboratories for nano-fabrication, metrology, and ultralow vibration experiments. IQC's headquarters, the Lazaridis Quantum-Nano Centre, was designed to facilitate interactions and includes ample space for discussions and collaborative research. IQC offers a unique interdisciplinary graduate program in Quantum Information to approximately 150 students. It also runs outreach programs, including the USEQIP summer school for undergraduates and the Quantum Innovators workshop for aspiring faculty candidates.

IQC sits within the Quantum Valley ecosystem, with partners including the Perimeter Institute for Theoretical Physics and Quantum Valley Ideas Laboratory; more than 250 researchers work within $1 \mathrm{~km}$ of IQC to advance the science, technology and commercial impact of quantum information. Investment in the Quantum Valley (governmental, industrial, philanthropic, and academic) now totals $\$ 1.8$ billion CAD, including the recent $\$ 76$ million CAD award by the Canada First Research Excellence Fund.

Attractive factors include stable funding, including an endowment of more than $\$ 100$ million CAD, the potential for significant faculty hiring (growth to 39 faculty members), and the established, internationally recognized excellence of IQC's comprehensive and multidisciplinary approach to quantum information. In addition, the Executive Director will have significant start-up funding to establish their research program.

\section{Exceptional candidates}

The successful candidate will share the Institute's core values for excellence in teaching and research; will have a bold strategic vision for the future of quantum information science and engineering; and will continue to build IQC through shared enthusiasm with faculty, staff, students, government, and industrial partners. The successful candidate will be able to recognize excellence broadly across the disciplines that comprise quantum information, and will lead the Institute's academic program, maintain a vision for research, strengthen the Institute's research infrastructure, and lead its hiring of exceptional new faculty. In addition, the successful candidate will promote collaborative efforts, support faculty led initiatives, and advance the Institute's commitment to faculty, student, and staff member inclusion, openness of processes, and equitable hiring and research practices.

Candidates for the position of Executive Director of IQC are $\mathrm{PhD}$ - level senior scholars in quantum information with leadership practice and at least 10 years' experience in an academic setting. They must be a leading authority in quantum information broadly, and have a demonstrated ability to maintain a research program of the highest calibre. IQC offers an internationally competitive salary.

The term of the appointment is five years, with the potential for one-time renewal. The successful candidate will hold a professorial appointment in an appropriate discipline within the Faculty of Mathematics, Science, or Engineering and will retain that position at the conclusion of their directorship. It is anticipated that the Executive Director will also be appointed to a position of Associate Faculty at the Perimeter Institute for Theoretical Physics.

Three reasons to apply: https://uwater10o.ca /faculty-association/why-waterloo.

The review of applications will begin November 15, 2018 and will continue until the position is filled.

The University of Waterloo regards diversity as an integral part of academic excellence and is committed to employment equity and accessibility for all employees. As such, we encourage applications from women, Indigenous (First Nations,Métis and Inuit) peoples, persons with disabilities, members of diverse gender identities, and others who may contribute to the further diversification of ideas. At Waterloo, you will have the opportunity to work across disciplines and collaborate with an international community of scholars and a diverse student body, situated in a rapidly growing community that has been termed a "hub of innovation". All qualified candidates are encouraged to apply; however, Canadians and permanent residents will receive priority in the recruitment process.

To Apply: Please send a curriculum vitae and a statement of interest in the position, in confidence, to Dr. Charmaine Dean, Vice-President University Research, at IQC .Search@uwater1oo.ca. 
If you have any questions regarding the position, the application process, assessment process, eligibility, or a request for accommodation during the hiring process, please contact Dr. Charmaine Dean, Vice-President University Research, at IQC.Search@uwater1oo.ca.

\section{CHINA}

\section{Tianjin University, China \\ Tenured/Tenure-Track/Postdoctoral Positions at the Center for Applied Mathematics}

Dozens of positions at all levels are available at the recently founded Center for Applied Mathematics, Tianjin University, China. We welcome applicants with backgrounds in pure mathematics, applied mathematics, statistics, computer science, bioinformatics, and other related fields. We also welcome applicants who are interested in practical projects with industries. Despite its name attached with an accent of applied mathematics, we also aim to create a strong presence of pure mathematics. Chinese citizenship is not required.

Light or no teaching load, adequate facilities, spacious office environment and strong research support. We are prepared to make quick and competitive offers to self-motivated hard workers, and to potential stars, rising stars, as well as shining stars.

The Center for Applied Mathematics, also known as the Tianjin Center for Applied Mathematics (TCAM), located by a lake in the central campus in a building protected as historical architecture, is jointly sponsored by the Tianjin municipal government and the university. The initiative to establish this center was taken by Professor S. S. Chern. Professor Molin Ge is the Honorary Director, Professor Zhiming Ma is the Director of the Advisory Board. Professor William Y. C. Chen serves as the Director.

TCAM plans to fill in fifty or more permanent faculty positions in the next few years. In addition, there are a number of temporary and visiting positions. We look forward to receiving your application or inquiry at any time. There are no deadlines.

Please send your resume to mathjobs@tju.edu.cn.

For more information, please visit cam.tju.edu .cn or contact Ms. Erica Liu at mathjobs@tju . edu .cn, telephone: 86-22-2740-6039.
When was the las time you visited the

AMS Bookstore?

\section{Spend smart.}

As an AMS member, enjoy free shipping, a $20 \%$ discount on most AMS titles, and a $25 \%$ discount on titles from MAA Press, an imprint of the American Mathematical Society. MAA members will enjoy a $25 \%$ discount on all AMS/MAA Press titles and $10 \%$ on all other AMS titles. Visit the Bookstore regularly for special monthly sales and discounts.

\section{Search better.}

Our full listing of books is conveniently organized by multiple criteria and searchable by various advanced options to help you find exactly what you need.

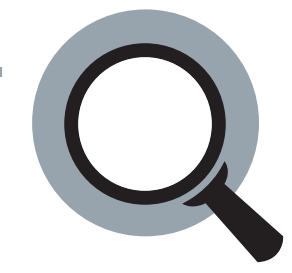

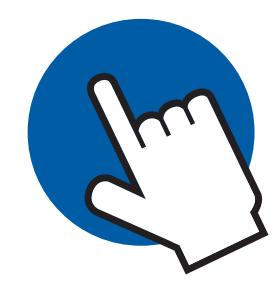

\section{Stay informed.}

The monthly AMS Bookstore New Releases email offers many benefits to subscribers. Stay up-to-date on all AMS publications and sales. Sign up

today at: ams.org/bookstore/keepinformed

\section{bookstore.ams.org}

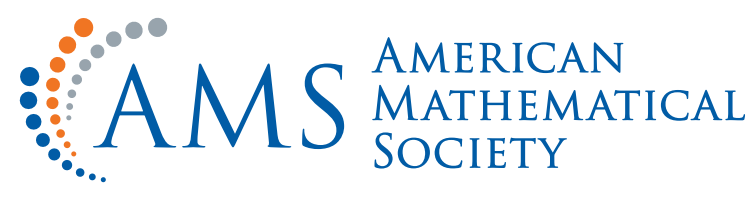




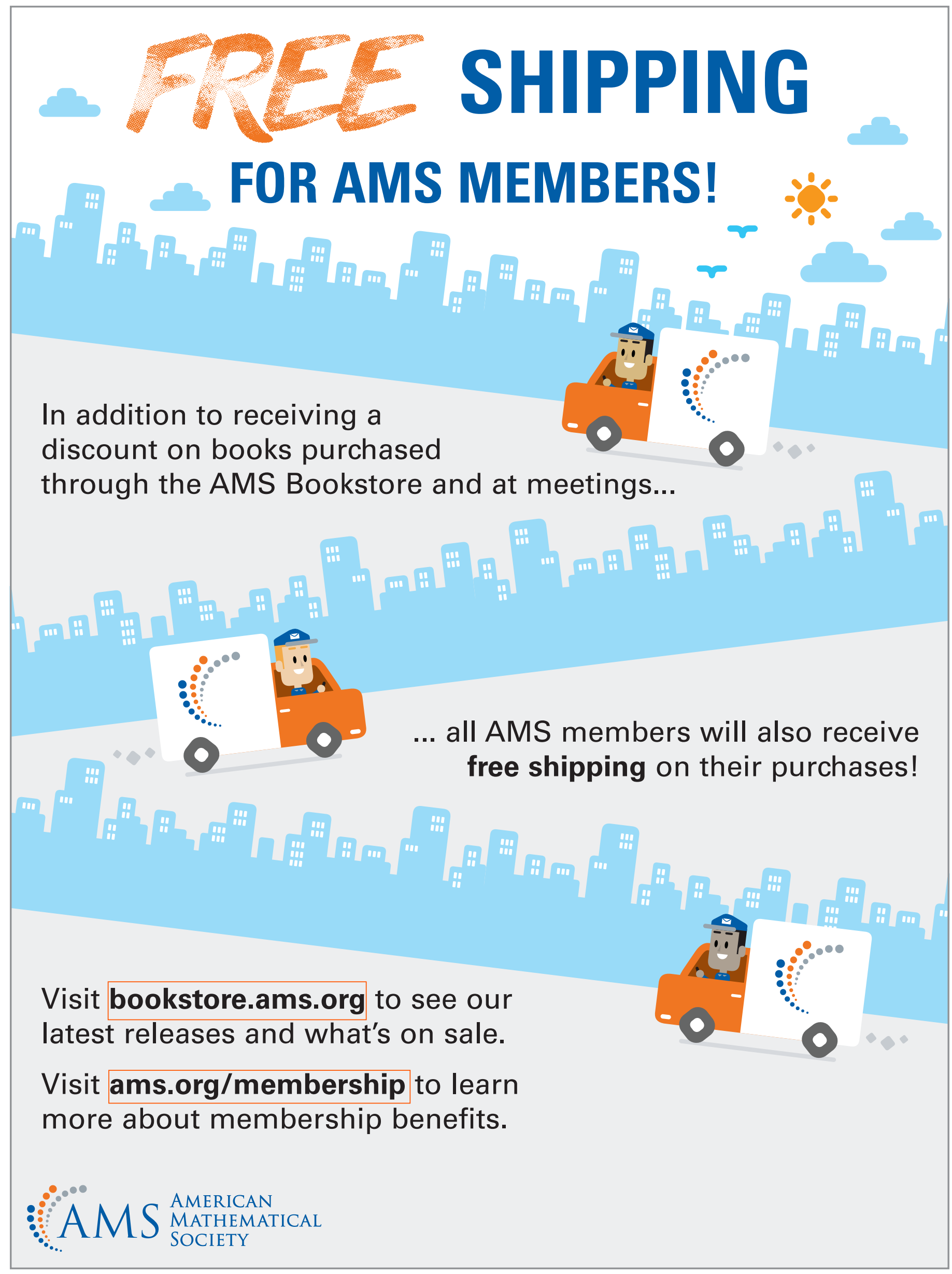




\section{New Books Offered by the AMS}

\section{Analysis}

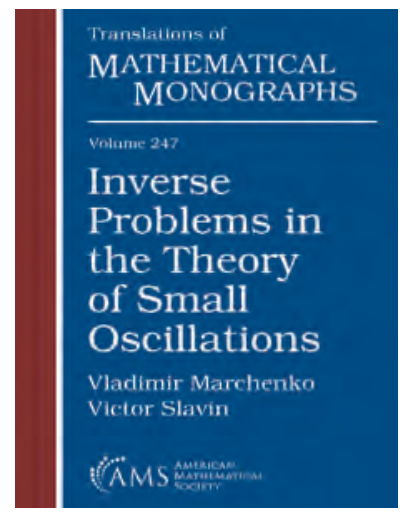

Inverse Problems in theTheory of Small Oscillations

Vladimir Marchenko, National Academy of Sciences of Ukraine, Kharkiv, Ukraine, and Victor Slavin, National Academy of Sciences of Ukraine, Kharkiv, Ukraine

This book treats inverse problems in the theory of small oscillations of systems with finitely many degrees of freedom, which requires finding the potential energy of a system from the observations of its oscillations. Since oscillations are small, the potential energy is given by a positive definite quadratic form whose matrix is called the matrix of potential energy. Hence, the problem is to find a matrix belonging to the class of all positive definite matrices. This is the main difference between inverse problems studied in this book and the inverse problems for discrete analogues of the Schrödinger operators, where only the class of tridiagonal Hermitian matrices are considered.

This item will also be of interest to those working in differential equations.

Translations of Mathematical Monographs, Volume 247 December 2018, 176 pages, Hardcover, ISBN: 978-1-47044890-5, LC 2018037179, 2010 Mathematics Subject Classification: 70F17, 65N21, 35P25, 35Q70, 65R32, List US\$126, AMS members US\$100.80, MAA members US\$113.40, Order code MMONO/247

bookstore. ams .org/mmono-247

\section{Applications}

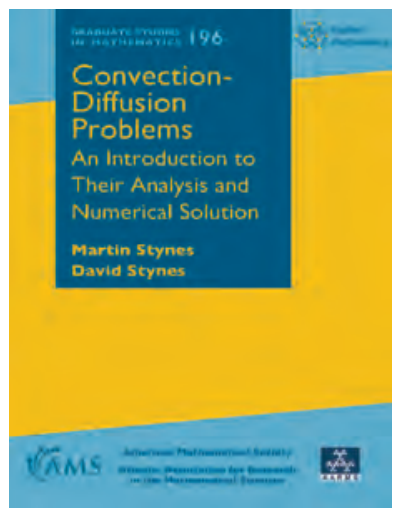

\section{Convection-Diffusion} Problems

An Introduction toTheir Analysis and Numerical Solution

Martin Stynes, Beijing Computational Science Research Center, China, and David Stynes, Cork Institute of Technology, Ireland

This lucid yet thorough account of convection-dominated, convection-diffusion problems and how to solve them numerically is meant for beginning graduate students and includes a large number of exercises and up-to-date bibliography that provides the reader with further reading.

Graduate Studies in Mathematics, Volume 196

December 2018, 156 pages, Hardcover, ISBN: 978-1-47044868-4, LC 2018035503, 2010 Mathematics Subject Classification: 65L11; 34B05, 34D15, 35B25, List US\$83, AMS members US\$66.40, MAA members US\$74.70, Order code GSM/196

bookstore.ams.org/gsm-196 


\section{New in Contemporary Mathematics}

\section{Analysis}

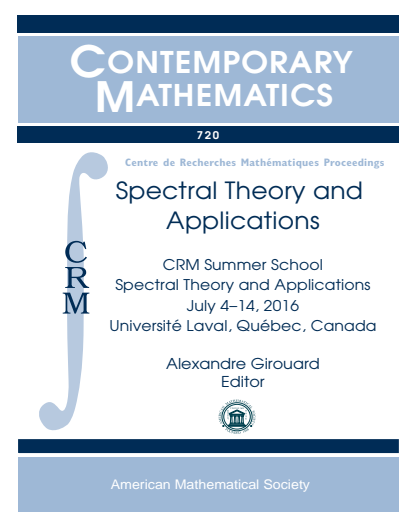

\section{SpectralTheory and} Applications

Alexandre Girouard, Université Laval, Québec, Canada, Editor

This book is a collection of lecture notes and survey papers based on the minicourses given by leading experts at the 2016 CRM Summer School on Spectral Theory and Applications held from July 4-14, 2016. The papers contained in the volume cover a broad variety of topics in spectral theory, starting from the fundamentals and highlighting its connections to PDEs, geometry, physics, and numerical analysis.

This item will also be of interest to those working in mathematical physics.

Contemporary Mathematics, Volume 720

November 2018, 212 pages, Softcover, ISBN: 978-14704-3556-1, LC 2018032989, 2010 Mathematics Subject Classification: 58Jxx, 35Pxx, 65Nxx, List US\$117, AMS members US\$93.60, MAA members US\$105.30, Order code CONM/720

bookstore. ams.org/conm-720

\section{Probability and Statistics}

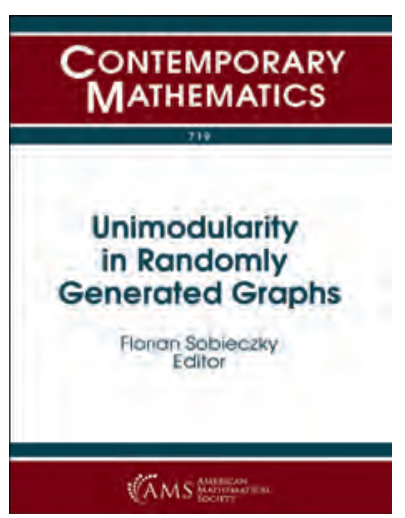

Unimodularity in Randomly Generated Graphs

Florian Sobieczky, Software Competence Center Hagenberg, Hagenberg in Mühlkreis, Austria, Editor

This volume contains the proceedings of the AMS Special Session on Unimodularity in Randomly Generated Graphs, held from October 8-9, 2016. This text tries to give an impression of the various fields in which the notion currently finds strong development and application: percolation theory, point processes, ergodic theory, and dynamical systems.

This item will also be of interest to those working in discrete mathematics and combinatorics.

Contemporary Mathematics, Volume 719

November 2018, 211 pages, Softcover, ISBN: 978-1-47043914-9, LC 2018027509, 2010 Mathematics Subject Classification: 60K35, 60K37, 60J35, 60C05, 60G55, 37A05, 37A35, List US\$117, AMS members US\$93.60, MAA members US\$105.30, Order code CONM/719

bookstore. ams.org/conm-719 


\section{Lisa Gaal Marcel Berger Cédric Villani}

Luis A. Caffarelli Tatiana Toro Dusa McDuff Shlomo Sternberg Terence Tao John Milnor Jean-Pierre Serre Victor Guillemin Karen Vogtmann Ingrid Daubechies Sigurdur Helgason

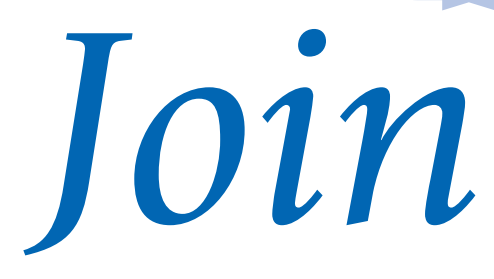

\section{the company} of great mathematicians

\section{BECOME AN AMS AUTHOR WHY PUBLISH WITH THE AMS?}

We are mathematicians. The AMS is one of the world's leading publishers of mathematical literature. As a professional society of mathematicians, we publish books and journals for the advancement of science and mathematics. Consequently, our publications meet the highest professional standards for their content and production.

Expertise. Our editorial boards consist of experienced mathematicians. The AMS production staff is talented and experienced at producing high-quality books and journals. The author support group consists of experts in TeX, graphics, and other aspects of the production of mathematical content.

Supporting mathematics. The AMS publication program is a part of our broader activities. The revenue it generates helps support our other professional activities. Thus, publishing with the AMS benefits the mathematical community.

Learn more at: www.ams.org/becomeanauthor

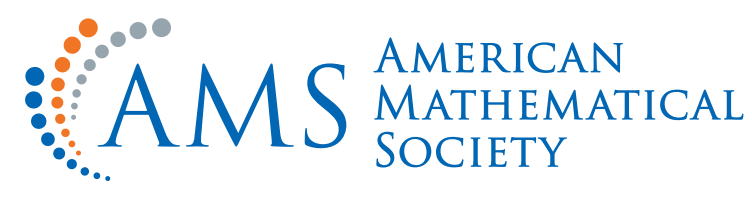




\section{American Associations for the Advancement of Science}

\section{Reinhard Laubenbacher, Secretary, Section A (Mathematics)}

The 2019 annual meeting of the American Association for the Advancement of Science (AAAS) will take place in Washington, DC, February 14-17, 2019. The 2019 theme for the meeting is Science Transcending Boundaries. AAAS is an organization one of whose broad goals is to enhance communication among scientists, engineers, and the public. It provides a tremendously useful resource for the mathematical sciences community, and the annual meeting is always packed with scientific symposia and keynote speakers that are of potential interest to mathematical scientists. The program for the upcoming meeting is particularly relevant to the mathematical sciences. A sampling of scientific symposia with mathematical and computational content include: Access to private and proprietary data: theorems and working prototypes; Mathematical modeling of diseases: translational approaches; Practical reproducible research: replicating scientific discoveries; Machine learning and statistics: applications in genomics and computer vision; Building quantum computers: why and how; Diversity + STEM $=$ X: Solving the equation for higher education and the workforce; AI and machine learning: industrial applications and social implications; Big data: overcoming challenges to facilitate data re-use; Machine learning and human language;
The science of quantum information: disentangling opportunities from the hype; Computational social science: the next decade; Fighting fake news: Views from social and computational science; How AI and knowledge centers are changing societal views of critical earth resources; Women in science: understanding what works; Refugees and immigrants: historic contributions to STEM. The full program, with speakers and abstracts, can be found at https://aaas.confex.com/aaas/2019/meetingapp.cgi/ModuleSessionsByDay/0.

In addition, there will be short presentations of the 2019 Class of AAAS fellows in the mathematical sciences, as part of the Section A (Mathematics) business meeting. This meeting, open to everybody, is a great opportunity to propose topics for scientific symposia for the 2020 meeting. 


\section{General Information Regarding Meetings \& Conferences of the AMS}

Speakers and Organizers: The Council has decreed that no paper, whether invited or contributed, may be listed in the program of a meeting of the Society unless an abstract of the paper has been received in Providence prior to the deadline.

Special Sessions: The number of Special Sessions at AMS meetings is limited. Special Sessions at annual meetings are held under the supervision of the Program Committee for National Meetings and, for sectional meetings, under the supervision of each Section Program Committee. They are administered by the Associate Secretary in charge of that meeting with staff assistance from the Meetings and Conferences Department in Providence. (See the list of Associate Secretaries on page 129 of this issue.)

Each person selected to give an Invited Address is also invited to generate a Special Session, either by personally organizing one or by having it organized by others. Proposals to organize a Special Session are sometimes solicited either by a program committee or by the Associate Secretary. Other proposals should be submitted to the associate secretary in charge of that meeting (who is an ex officio member of the program committee) at the address listed on page 90 of this issue. These proposals must be in the hands of the Associate Secretary at least seven months (for sectional meetings) or nine months (for national meetings) prior to the meeting at which the Special Session is to be held in order that the committee may consider all the proposals for Special Sessions simultaneously. Special Sessions must be announced in the Notices in a timely fashion so that any Society member who so wishes may submit an abstract for consideration for presentation in the Special Session.

Talks in Special Sessions are usually limited to twenty minutes; however, organizers who wish to allocate more time to individual speakers may do so within certain limits. A great many of the papers presented in Special Sessions at meetings of the Society are invited papers, but any member of the Society who wishes to do so may submit an abstract for consideration for presentation in a Special Session. Contributors should know that there is a limit to the size of a single Special Session, so sometimes all places are filled by invitation. An author may speak in more than one Special Session at the same meeting. However, multiple talks given by the same author at a meeting must be distinct and have distinct abstracts. Papers submitted for consideration for inclusion in Special Sessions but not accepted will receive consideration for a Contributed Paper Session, unless specific instructions to the contrary are given.

The Society reserves the right of first refusal for the publication of proceedings of any Special Session. If published by the AMS, these proceedings appear in the book series Contemporary Mathematics. For more detailed information on organizing a Special Session, see WwW . ams.org /meetings/meet-specialsessionmanual.htm1.

Contributed Papers: The Society also accepts abstracts for ten-minute contributed papers. These abstracts will be grouped by related Mathematical Reviews subject classifications into sessions to the extent possible. The title and author of each paper accepted and the time of presentation will be listed in the program of the meeting. Although an individual may present only one ten-minute contributed paper at a meeting, any combination of joint authorship may be accepted, provided no individual speaks more than once.

Other Sessions: In accordance with policy established by the AMS Committee on Meetings and Conferences, mathematicians interested in organizing a session (for either an annual or a sectional meeting) on employment opportunities inside or outside academia for young mathematicians should contact the Associate Secretary for the meeting with a proposal by the stated deadline. Also, potential organizers for Poster Sessions (for an annual meeting) on a topic of choice should contact the associate secretary before the deadline. 


\section{MEETINGS \& CONFERENCES}

Abstracts: Abstracts for all papers must be received by the meeting coordinator in Providence by the stated deadline. Unfortunately, late papers cannot be accommodated.

Submission Procedures: Visit the Meetings and Conferences homepage on the Web at www.ams.org /meetings and select "Submit Abstracts."

\section{Site Selection for Sectional Meetings}

Sectional meeting sites are recommended by the Associate Secretary for the section and approved by the Secretariat. Recommendations are usually made eighteen to twenty-four months in advance. Host departments supply local information,twenty-five to thirty rooms equipped with computer projection systems and chalkboards or whiteboards for Contributed Paper Sessions and Special Sessions, a large auditorium equipped with a computer projection system and mikes for the Invited Addresses, space for registration activities and an AMS book exhibit, and registration clerks. The Society will partially reimburse for expenses needed to run these meetings successfully.

For more information, contact the Associate Secretary for the section. 


\section{Meetings \& Conferences of the AMS January Table of Contents}

The Meetings and Conferences section of the Notices gives information on all AMS meetings and conferences approved by press time for this issue. Please refer to the page numbers cited on this page for more detailed information on each event.

Invited Speakers and Special Sessions are listed as soon as they are approved by the cognizant program committee; the codes listed are needed for electronic abstract submission. For some meetings the list may be incomplete. Information in this issue may be dated.

The most up-to-date meeting and conference information can be found online at: www . ams . org/meetings.

Important Information About AMS Meetings: Potential organizers, speakers, and hosts should refer to page 127 in the January 2019 issue of the Notices for general information regarding participation in AMS meetings and conferences.

Abstracts: Speakers should submit abstracts on the easy-to-use interactive Web form. No knowledge of LATEX is necessary to submit an electronic form, although those who use LATEX may submit abstracts with such coding, and all math displays and similarily coded material (such as accent marks in text) must be typeset in LTEX. Visit WwW . ams. org/cgi-bin/abstracts/abstract.p1. Questions about abstracts may be sent to abs-info@ams . org. Close attention should be paid to specified deadlines in this issue. Unfortunately, late abstracts cannot be accommodated.

\section{Associate Secretaries of the AMS}

Central Section: Georgia Benkart, University of Wisconsin-Madison, Department of Mathematics, 480 Lincoln Drive, Madison, WI 53706-1388; email: benkart@math . wi sc . edu; telephone: 608-263-4283.

Eastern Section: Steven H. Weintraub, Department of Mathematics, Lehigh University, Bethlehem, PA 180153174; email: steve.weintraub@1ehigh. edu; telephone: 610-758-3717.

Southeastern Section: Brian D. Boe, Department of Mathematics, University of Georgia, 220 D W Brooks Drive, Athens, GA 30602-7403, email: brian@math . uga . edu; telephone: 706-542-2547.

Western Section: Michel L. Lapidus, Department of Mathematics, University of California, Surge Bldg., Riverside, CA 92521-0135; email: 1apidus@math. ucr . edu; telephone: 951-827-5910.

\section{Meetings in this Issue}

\begin{tabular}{|c|c|c|}
\hline January 16-19 & Baltimore, Maryland & p. 130 \\
\hline March 15-17 & Auburn, Alabama & p. 134 \\
\hline March 22-24 & Honolulu, Hawai'i & p. 140 \\
\hline April 13-14 & Hartford, Connecticut & p. 148 \\
\hline June $10-13$ & Quy Nhon City, Vietnam & p. 150 \\
\hline September 14-15 & Madison, Wisconsin & p. 150 \\
\hline October $12-13$ & Binghamton, New York & p. 151 \\
\hline November 2-3 & Gainesville, Florida & p. 151 \\
\hline November 9-10 & Riverside, California & p. 152 \\
\hline
\end{tabular}
2020

January $15-18$

Denver, Colorado

p. 152

March 13-15

Charlottesville, Virginia

p. 152

March 21-22

Medford, Massachusetts

p. 153

May 2-3

Fresno, California

p. 153

September 12-13 El Paso, Texas

p. 153

October 3-4

State College, Pennsylvania p. 153

October 24-25

Salt Lake City, Utah

p. 154

2021

$\begin{array}{lll}\text { January 6-9 } & \text { Washington, DC } & \text { p. } 154 \\ \text { July 5-9 } & \text { Grenoble, France } & \text { p. } 154 \\ \text { July 19-23 } & \text { Buenos Aires, Argentina } & \text { p. } 154 \\ \text { October 9-10 } & \text { Omaha, Nebraska } & \text { p. } 155\end{array}$

2022

January 5-8 Seattle, Washington

p. 155

\section{3}

January 4-7 Boston, Massachusetts

p. 155

See https://www. ams.org/meetings for the most up-to-date information on the meetings and conferences that we offer. 


\section{Meetings \& Conferences of the AMS}

IMPORTANT INFORMATION REGARDING MEETINGS PROGRAMS: AMS Sectional Meeting programs do not appear in the print version of the Notices. However, comprehensive and continually updated meeting and program information with links to the abstract for each talk can be found on the AMS website. See www . ams .org/meetings/.

\section{Baltimore, Maryland}

Baltimore Convention Center, Hilton Baltimore, and Baltimore Marriott Inner Harbor Hotel

\section{January 16-19, 2019}

Wednesday - Saturday

\section{Meeting \#1145}

Joint Mathematics Meetings, including the 125th Annual Meeting of the AMS, 102nd Annual Meeting of the Mathematical Association of America (MAA), annual meetings of the Association for Women in Mathematics (AWM) and the National Association of Mathematicians (NAM), and the winter meeting of the Association for Symbolic Logic (ASL), with sessions contributed by the Society for Industrial and Applied Mathematics (SIAM).
Associate Secretary: Steven H. Weintraub

Announcement issue of Notices: October 2018

Program first available on AMS website: November 1, 2018 Issue of Abstracts: Volume 40, Issue 1

\section{Deadlines}

For organizers: Expired

For abstracts: Expired

The scientific information listed below may be dated. For the latest information, see www . ams . org/amsmtgs/nationa1 . htm 1 .

\section{Joint Invited Addresses}

Sarah Koch, University of Michigan, What is the shape of a rational map? (AMS-MAA Invited Address).

Bryna Kra, Northwestern University, Dynamics of systems with low complexity (AWM-AMS Noether Lecture).

Cathy O'Neil, ORCAA, Big data, inequality, and democracy (MAA-AMS-SIAM Gerald and Judith Porter Public Lecture).

Daniel A Spielman, Yale University, Miracles of Algebraic Graph Theory (AMS-MAA Invited Address).

\section{AMS Invited Addresses}

Jesús A. De Loera, University of California, Davis, Algebraic, Geometric, and Topological Methods in Optimization.

Benedict H. Gross, University of California San Diego, Complex multiplication: past, present, future (AMS Colloquium Lectures: Lecture I).

Benedict H. Gross, University of California San Diego, Complex multiplication: past, present, future (AMS Colloquium Lectures: Lecture II).

Benedict H. Gross, University of California San Diego, Complex multiplication: past, present, future (AMS Colloquium Lectures: Lecture III).

Peter Ozsvath, Princeton University, From knots to symplectic geometry and algebra. 
Lior Pachter, California Institute of Technology, A mathematical introduction to the molecular biology of the cell. Karen Hunger Parshall, University of Virginia, The roaring twenties in American mathematics.

Alan S. Perelson, Los Alamos National Laboratory, Immunology for mathematicians (AMS Josiah Willard Gibbs Lecture). Lillian B. Pierce, Duke University, On torsion subgroups in class groups of number fields.

\section{AMS Special Sessions}

Some sessions are cosponsored with other organizations. These are noted within the parenthesis at the end of each listing, where applicable.

25 years of Conferences for African-American Researchers in the Mathematical Sciences (CAARMS times 25), William A. Massey, Princeton University.

A Showcase of Number Theory at Undergraduate Institutions, Adriana Salerno, Bates College, and Lola Thompson, Oberlin College.

Advances and Applications in Integral and Differential Equations, Jeffrey T. Neugebauer, Eastern Kentucky University, and Min Wang, Kennesaw State University.

Advances by Early Career Women in Discrete Mathematics, Jessalyn Bolkema, State University of New York at Oswego, and Jessica De Silva, California State University, Stanislaus.

Advances in Operator Theory, Operator Algebras, and Operator Semigroups, Joseph Ball, Virginia Tech, Marat Markin, California State University, Fresno, Igor Nikolaev, St. John's University, and Ilya Spitkovsky, New York University, Abu Dhabi.

Advances in Quantum Walks, Quantum Simulations, and Related Quantum Theory, Radhakrishnan Balu, US Army Research Lab, Chaobin Liu, Bowie State University, and Takuya Machida, Nihon University, Japan.

Agent-based Modeling in Biological and Social Systems (a Mathematics Research Communities Session), Maryann Hohn, University of California Santa Barbara, Angelika Manhart, Imperial College, London, Christopher Miles, Courant Institute, New York University, and Cole Zmurchok, Vanderbilt University.

Algebraic Structures Motivated by Knot Theory, Mikhail Khovanov, Columbia University, and Jozef H. Przytycki and Alexander Shumakovitch, George Washington University.

Algebraic and Geometric Methods in Discrete Optimization, Amitabh Basu, Johns Hopkins University, and Jesus De Loera, University of California, Davis.

Algebraic, Discrete, Topological and Stochastic Approaches to Modeling in Mathematical Biology, Olcay Akman, Illinois State University, Timothy D. Comar, Benedictine University, Daniel Hrozencik, Chicago State University, and Raina Robeva, Sweet Briar College.

Algorithmic Dimensions and Fractal Geometry, Jack H. Lutz, Iowa State University, and Elvira Mayordomo, University of Zaragoza, Spain (AMS-ASL).

Analysis and Geometry of Nonlinear Evolution Equations, Marius Beceanu, University at Albany, State University of New York, and Dan-Andrei Geba, University of Rochester.

Analysis of Fractional, Stochastic, and Hybrid Dynamic Systems with Applications, John R. Graef, University of Tennessee at Chattanooga, G. S. Ladde, University of South Florida, and A. S. Vatsala, University of Louisiana at Lafayette.

Analytic Number Theory, Thomas A. Hulse, Boston College, Angel V. Kumchev and Nathan McNew, Towson University, and John Miller, The Johns Hopkins University.

Arithmetic Statistics, Michael Chou and Robert Lemke Oliver, Tufts University, and Ari Shnidman, Center for Communications Research-Princeton.

Bifurcations of Difference Equations and Discrete Dynamical Systems with Applications, Arzu Bilgin, Recep Tayyip Erdogan University, Turkey, and Toufik Khyat, Trinity College.

Commutative Ring Theory: Research for Undergraduate and Early Graduate Students, Nicholas Baeth, Franklin and Marshall College, and Branden Stone, Hamilton College.

Continued Fractions, Geremías Polanco Encarnación, Hampshire College, James McLaughlin, West Chester University,

Barry Smith, Lebanon Valley College, and Nancy J. Wyshinski, Trinity College.

Counting Methods in Number Theory, Lillian Pierce, Duke University, Arindam Roy, Rice University, and Jiuya Wang, University of Wisconsin.

Definability and Decidability Problems in Number Theory, Kirsten Eisenträger, Pennsylvania State University, Deidre Haskell, McMaster University, Ontario, Canada, Jennifer Park, University of Michigan, and Alexandra Shlapentokh, East Carolina University (AMS-ASL).

Differential Equations on Fractals, Patricia Alonso-Ruiz, University of Connecticut, Joe Chen, Colgate University, Luke Rogers, University of Connecticut, Robert Strichartz, Cornell University, and Alexander Teplyaev, University of Connecticut. 


\section{MEETINGS \& CONFERENCES}

Enumerative Combinatorics, Miklos Bona, University of Florida, and Cheyne Homberger, University of Maryland, Baltimore County.

Financial Mathematics, Maxim Bichuch, Johns Hopkins University, Anja Richter, Baruch College, City University of New York, and Stephan Sturm, Worcester Polytechnic Institute.

Geometric and Topological Combinatorics, Anastasia Chavez and Jamie Haddock, University of California, Davis, and Annie Raymond, University of Massachusetts, Amherst.

Geometric and Topological Generalization of Groups, Amrita Acharyya, University of Toledo, and Bikash C. Das, University of North Georgia.

Geometry Labs United: Research, Visualization, and Outreach, Marianne Korten, Kansas State University, and Sean Lawton and Anton Lukyanenko, George Mason University.

Geometry and Dynamics of Continued Fractions, Anton Lukyanenko, George Mason University, and Joseph Vandehey, Ohio State University.

Geometry of Representation Spaces, Sean Lawton, George Mason University, Chris Manon, University of Kentucky, and Daniel Ramras, Indiana University-Purdue University Indianapolis.

Group Representation Theory and Character Theory, Mohammad Reza Darafsheh, University of Tehran, Iran, and Manouchehr Misaghian, Prairie View A\&M University.

Harmonic Analysis, Partial Differential Equations, and Applications, Russell Brown, University of Kentucky, and Irina Mitrea, Temple University.

Harmonic Analysis: Recent Developments on Oscillatory Integrals (a Mathematics Research Communities Session), Xiumin Du, University of Maryland, Taryn C. Flock, University of Massachusetts Amherst, and Yakun Xi, University of Rochester. History of Mathematics, Sloan Despeaux, Western Carolina University, Jemma Lorenat, Pitzer College, Daniel E. Otero, Xavier University, and Adrian Rice, Randolph-Macon College (AMS-MAA-ICHM).

Hopf Algebras and Tensor Categories, Siu-Hung Ng, Louisiana State University, Julia Plavnik, Texas A\&M University, and Henry Tucker, University of California, San Diego.

How to Guard an Art Gallery and Other Discrete Mathematical Adventures (In Memory of T. S. Michael, 1960 to 2016), Joseph

Bonin, The George Washington University, Carolyn Chun, US Naval Academy, and Nancy Neudauer, Pacific University. If You Build It They Will Come: Presentations by Scholars in the National Alliance for Doctoral Studies in the Mathematical

Sciences, David Goldberg, Purdue University, and Phil Kutzko, University of Iowa.

Latinx in Math, Alexander Diaz-Lopez, Villanova University, Laura Escobar, University of Illinois, and Juanita Pinzón-

Caicedo, North Carolina State University.

Lattice Path Combinatorics and Applications, Christian Krattenthaler, University of Vienna, Austria, and Alan Krinik and

Randall J. Swift, California State Polytechnic University.

Localization and Delocalization for Disordered Quantum Systems, Peter D. Hislop, University of Kentucky, Christoph A.

Marx, Oberlin College, and Jeffery Schenker, Michigan State University.

Low Complexity Models in Data Analysis and Machine Learning, Emily J. King, University of Bremen, Germany, Nate

Strawn, Georgetown University, and Soledad Villar, New York University.

Mappings on Metric and Banach Spaces with Applications to Fixed Point Theory, Torrey M. Gallagher, Bucknell University, and Christopher J. Lennard, University of Pittsburgh.

Mathematical Analysis in Fluid Dynamics, Yanqiu Guo, Florida International University, Jinkai Li, South China Normal University, Jing Tian, Towson University, and Yuncheng You, University of South Florida.

Mathematical Investigations of Spatial Ecology and Epidemiology, Leah Shaw and Junping Shi, College of William and

Mary, and Zhisheng Shuai, University of Central Florida.

Mathematical Models in Ecology, Epidemiology, and Medicine, Richard Schugart, Western Kentucky University, and Najat Ziyadi, Morgan State University.

Mathematicians at Sea (in the Sky, or on Land): Defense Applications of Mathematics, Tegan Emerson, Timothy Doster, and George Stantchev, Naval Research Laboratory.

Mathematics in the Realm of Cyber Research, Daniel Bennett, Army Cyber Institute, Paul Goethals, United States Military Academy, and Natalie Scala, Towson University.

Mathematics of Coding Theory and Applications, Hiram Lopez-Valdez and Felice Manganiello, Clemson University, and

Gretchen L. Matthews, Virginia Tech.

Multiscale Problems in the Calculus of Variations, Elisa Davoli, University of Vienna, Austria, and Rita Ferreira, King Abdullah University of Science and Technology, Saudi Arabia.

Natural Resources Modeling, Julie Blackwood, Williams College, and Shandelle M. Henson, Andrews University. 
Network Science, David Burstein, Swarthmore College, Franklin Kenter, United States Naval Academy, and Feng 'Bill' Shi, University of North Carolina.

New Directions in the Theory of Complex Multiplication, Henri Darmon, McGill University, Samit Dasgupta, University of California, Santa Cruz, and Benedict Gross, Harvard University.

Nonlinear Evolution Equations and Their Applications, Mingchao Cai, Morgan State University, Gisele Mophou Loudjom, University of French West Indies, Guadeloupe, France, and Gaston N'Guerekata, Alexander Pankov, Xuming Xie, and Guoping Zhang, Morgan State University.

Not KNerds: A Community for Knot Theory, Moshe Cohen, Vassar College, Elizabeth Denne, Washington and Lee University, and Adam Lowrance, Vassar College.

Number Theoretic Methods in Hyperbolic Geometry (a Mathematics Research Communities Session), Samantha Fairchild, University of Washington, Junxian Li, Universität Göttingen, and Richard Vradenburgh, University of Virginia.

Number Theory, Arithmetic Geometry, and Computation, Brendan Hassett, Brown University, Drew Sutherland, Massachusetts Institute of Technology, and John Voight, Dartmouth College.

Numerical Methods for PDEs and Applications, Wenrui Hao, Qingguo Hong, and Jinchao Xu, Pennsylvania State University.

Optimal Methods in Applicable Analysis: Variational Inequalities, Low Rank Matrix Approximations, Systems Engineering, Cyber Security, Aritra Dutta, King Abdullah University of Science and Technology, Saudi Arabia, Ram Mohapatra, University of Central Florida, Gayatri Pany, Singapore University of Technology and Design, Singapore, and Nabin Kumar Sahu, Dhirubhai Ambani Institute of Information and Communication Technology, India.

Orthogonal Polynomials, Quantum Probability, Harmonic and Stochastic Analysis, Nobuhiro Asai, Aichi University of Education, Kariya, Japan, Rodica Costin, The Ohio State University, Aurel I. Stan, The Ohio State University at Marion, and Hiroaki Yoshida, Ochanomizu University, Tokyo, Japan.

Partition Theory and Related Topics, Dennis Eichhorn, University of California, Irvine, Tim Huber, University of Texas, Rio Grande Valley, and Amita Malik, Rutgers University.

Problems in Partial Differential Equations, Alex Himonas, University of Notre Dame, and Curtis Holliman, The Catholic University of America.

Quantum Symmetries: Subfactors and Fusion Categories (a Mathematics Research Communities Session), Cain Edie-Michell and Lauren Ruth, Vanderbilt University, and Yilong Wang, Louisiana State University.

Quaternions, Terrence Blackman, Medgar Evers College, City University of New York, and Johannes Familton and

Chris McCarthy, Borough of Manhattan Community College, City University of New York.

Recent Advancements in Mathematical Modeling of Cancer, Kamila Larripa, Humboldt State University, and Hwayeon Ryu, University of Hartford.

Recent Advances and Trends in Computable Structure Theory (in honor of J. Remmel), Jennifer Chubb, University of San Francisco, and Tim McNicholl, Iowa State University.

Recent Advances in Biological Modeling and Related Dynamical Analysis, Joshi Raj Hem, Xavier University, and Yanyu Xiao, University of Cincinnati.

Recent Advances in Homological and Commutative Algebra, Neil Epstein, George Mason University, Claudiu Raicu, Notre Dame University, and Alexandra Seceleanu, University of Nebraska.

Recent Advances in Inverse Problems and Imaging, Kui Ren, University of Texas at Austin, and Yang Yang, Michigan State University.

Recent Advances in Regularity Lemmas, Gabriel Conant, University of Notre Dame, Rehana Patel, and Julia Wolf, University of Bristol, UK.

Recent Progress in Multivariable Operator Theory, Dmitry Kaliuzhnyi-Verbovetsky and Hugo Woerdeman, Drexel University.

Research in Mathematics by Early Career Graduate Students, Marat Markin, Morgan Rodgers, Khang Tran, and Oscar Vega, California State University, Fresno.

Research in Mathematics by Undergraduates and Students in Post-Baccalaureate Programs, Darren A. Narayan, Rochester Institute of Technology, Khang Tran, California State University, Fresno, Mark David Ward, Purdue University, and John Wierman, The Johns Hopkins University (AMS-MAA-SIAM).

Riordan Arrays, Alexander Burstein and Dennis Davenport, Howard University, Asamoah Nkwanta, Morgan State University, Lou Shapiro, Howard University, and Leon Woodson, Morgan State University.

Statistical, Variational, and Learning Techniques in Image Analysis and their Applications to Biomedical, Hyperspectral, and Other Imaging, Justin Marks, Gonzaga University, Laramie Paxton, Washington State University, and Viktoria Taroudaki, Eastern Washington University. 


\section{MEETINGS \& CONFERENCES}

Stochastic Analysis and Applications in Finance, Actuarial Science and Related Fields, Julius N. Esunge, University of Mary

Washington, See Keong Lee, University of the Sciences, Malaysia, and Aurel I. Stan, The Ohio State University at Marion.

Stochastic Differential Equations and Applications, Carey Caginalp, University of Pittsburgh.

Symbolic Dynamics, Van Cyr, Bucknell University, and Bryna Kra, Northwestern University.

The Mathematics of Gravity and Light (a Mathematics Research Communities Session), Sougata Dhar, University of Maine,

Chad R. Mangum, Niagara University, and Nadine Stritzelberger, University of Waterloo.

The Mathematics of Historically Black Colleges and Universities (HBCUs) in the Mid-Atlantic, Edray Goins, Purdue University, Janis Oldham, North Carolina A\&T, Talithia Washington, Howard University, and Scott Williams, University at Buffalo, State University of New York.

Topological Data Analysis: Theory and Applications, Justin Curry, University at Albany, State University of New York, Mikael Vejdemo-Johansson, College of Staten Island, City University of New York, and Sara Kalisnik Verovsek, Wesleyan University.

Topology, Structure and Symmetry in Graph Theory, Lowell Abrams, George Washington University, and Mark Ellingham, Vanderbilt University.

Using Modeling to Motivate the Study of Differential Equations, Robert Kennedy, Centennial High School, Ellicott City MD, Audrey Malagon, Virginia Wesleyan University, Brian Winkel, SIMIODE, Cornwall NY, and Dina Yagodich, Frederick Community College.

Women in Topology, Jocelyn Bell, Hobart and William Smith Colleges, Rosemary Guzman, University of Chicago, Candice Price, University of San Diego, and Arunima Ray, Max Planck Institute for Mathematics, Germany.

\section{Auburn, Alabama}

\section{Auburn University}

March 15-17, 2019

Friday - Sunday

\section{Meeting \#1146}

Southeastern Section

Associate Secretary: Brian D. Boe
Announcement issue of Notices: January 2019

Program first available on AMS website: January 31, 2019 Issue of Abstracts: Volume 40, Issue 2

\section{Deadlines}

For organizers: Expired

For abstracts: January 29, 2019

The scientific information listed below may be dated. For the latest information, see www . ams . org/amsmtgs/sectiona1 . htm1.

\section{Invited Addresses}

Grigoriy Blekherman, Georgia Institute of Technology, Do sums of squares dream of free resolutions?

Carina Curto, Pennsylvania State University, To be announced.

Ming Liao, Auburn University, Invariant Markov processes under actions of Lie groups.

\section{Special Sessions}

If you are volunteering to speak in a Special Session, you should send your abstract as early as possible via the abstract submission form found at http://www. ams . org/cgi-bin/abstracts/abstract.p1.

Algebraic and Discrete Methods in Mathematical Biology (Code: SS 21A), Carina Curto, The Pennsylvania State University, Katherine Morrison, University of Northern Colorado, and Nora Youngs, Colby College.

Applications of Algebraic Geometry (Code: SS 25A), Greg Blekherman, Georgia Institute of Technology, Michael Burr, Clemson University, and Tianran Chen, Auburn University at Montgomery.

Clustering Methods and Applications (Code: SS 23A), Benjamin McLaughlin, Naval Surface Warfare Center Panama City

Division (NSWCPCD), and Sung Ha Kang, Georgia Institute of Technology.

Combinatorial Matrix Theory (Code: SS 2A), Zhongshan Li, Georgia State University, and Xavier Martínez-Rivera, Auburn University.

Commutative and Combinatorial Algebra (Code: SS 3A), Selvi Kara Beyarslan, University of South Alabama, and Alessandra Costantini, Purdue University. 
Developments in Commutative Algebra (Code: SS 1A), Eloísa Grifo, University of Michigan, and Patricia Klein, University of Kentucky.

Differential Equations in Mathematical Biology (Code: SS 7A), Guihong Fan, Columbus State University, Zhongwei Shen, University of Alberta, and Xiaoxia Xie, Idaho State University.

Discrete and Convex Geometry (Code: SS 17A), Andras Bezdek, Auburn University, Ferenc Fodor, University of Szeged, and Wlodzimierz Kuperberg, Auburn University.

Evolution Equations and Applications (Code: SS 9A), Dmitry Glotov, Wenxian Shen, and Paul G. Schmidt, Auburn University.

Experimental Mathematics in Number Theory, Analysis, and Combinatorics (Code: SS 6A), Amita Malik, Rutgers University, and Armin Straub, University of South Alabama.

Geometric Flows and Minimal Surfaces (Code: SS 20A), Theodora Bourni, University of Tennessee, and Giuseppe Tinaglia, King's College London and University of Tennessee.

Geometric Methods in Representation Theory (Code: SS 15A), Jiuzu Hong and Shrawan Kumar, University of North Carolina, Chapel Hill, and Yiqiang Li, University at Buffalo, the State University of New York.

Geometric and Combinatorial Aspects of Representation Theory (Code: SS 19A), Mark Colarusso, University of South Alabama, and Jonas Hartwig, Iowa State University.

Geometry and Topology of Low Dimensional Manifolds, and Their Invariants (Code: SS 13A), John Etnyre, Georgia Institute of Technology, Bulent Tosun, University of Alabama, and Shea Vela-Vick, Louisiana State University.

Graph Theory in Honor of Robert E. Jamison's 70th Birthday (Code: SS 4A), Robert A Beeler, East Tennessee State University, Gretchen Matthews, Virginia Tech, and Beth Novick, Clemson University.

Hopf Algebras and Their Applications (Code: SS 10A), Robert Underwood, Auburn University at Montgomery, and Alan Koch, Agnes Scott College.

Mapping Class Groups (Code: SS 27A), Joan Birman, Columbia University, and Kevin Kordek and Dan Margalit, Georgia Institute of Technology.

Mathematical Analysis and Control Theory of Coupled Partial Differential Equation Models (Code: SS 11A), George Avalos and Pelin Gu"ven Geredeli, University of Nebraska-Lincoln, and László Kindrat, University of New Hampshire.

Nonlinear Reaction-Diffusion Equations and Their Applications (Code: SS 18A), Jerome Goddard,II, Auburn University at Montgomery, Nsoki Mavinga, Swarthmore College, Quinn Morris, Appalachian State University, and R. Shivaji, University of North Carolina at Greensboro.

Probability and Stochastic Processes (Code: SS 5A), Ming Liao, Erkan Nane, and Jerzy Szulga, Auburn University.

Random Discrete Structures (Code: SS 24A), Lutz P Warnke, Georgia Institute of Technology, and Xavier Pérez-Giménez, University of Nebraska-Lincoln.

Recent Advances in Coarse Geometry (Code: SS 12A), Jerzy Dydak, University of Tennessee.

Recent Advances in Numerical Methods for PDEs and PDE-constrained Optimization (Code: SS 26A), Yanzhao Cao, Thi-

Thao-Phuong Hoang, and Junshan Lin, Auburn University.

Recent Developments in Graph Theory (Code: SS 16A), Xiaofeng Gu, Jeong-Hyun Kang, David Leach, and Rui Xu, University of West Georgia.

Representations of Lie Algebras, Algebraic Groups, and Quantum Groups (Code: SS 8A), Joerg Feldvoss, University of South Alabama, Lauren Grimley, Spring Hill College, and Cornelius Pillen, University of South Alabama.

The Modeling and Analysis of Spatially Extended Structures (Code: SS 22A), Shibin Dai, University of Alabama, Keith Promislow, Michigan State University, and Qiliang Wu, Ohio University.

Topological Data Analysis, Statistics and Applications (Code: SS 14A), Yu-Min Chung, University of North Carolina at Greensboro, and Vasileios Maroulas, University of Tennessee.

\section{Accommodations}

Participants should make their own arrangements directly with the hotel of their choice. Special discounted rates were negotiated with the hotels listed below. Rates quoted do not include the Alabama state hotel tax (13\%), local taxes and hotel fees may apply. Participants must state that they are with the American Mathematical Society's (AMS) Spring Southeast Sectional Meeting to receive the discounted rate. The AMS is not responsible for rate changes or for the quality of the accommodations. Hotels have varying cancellation and early checkout penalties; be sure to ask for details.

The Hotel at Auburn University (directly across the street from Mell Classroom Building), 241 S College Street, Auburn, AL 36830 ; (334) 821-8200; wWw. auhcc. com. Rates are US\$142 per night for a double or king room and US\$182 per night for an executive room. To make a reservation online, please use this link: https://gc.synxis.com /?Hote1=75989\&temp1 ate=RBE\&she11=RBE\&Group=190315MATH\&ar rive=03/13/2019. The Hotel at Auburn 


\section{MEETINGS \& CONFERENCES}

University offers complimentary WiFi and a 24 hour fitness center. Overnight self-parking is US $\$ 5$ per night and overnight valet parking is US\$15 per night. Ariccia Trattoria, the hotel's Italian and Mediterranean inspired restaurant, offers fresh pizzas, pastas and grill entrees for dinner seven nights a week in addition to a daily breakfast buffet and lunch a la carte menu. Their is also a jazz lounge and lobby bar on-site.

This property is a shuttle stop for Groome Transportation which services between Auburn and Atlanta International Airport; https://groometransportation. com/auburn/\#gt-auburnsched-1.

The deadline for reservations at this rate is February 11, 2019.

TownePlace Suites Auburn (1.7 miles from Mell Classroom Building), 1117 S College Street, Auburn, AL $36830 ;(334)$ 446-8390; https: //www. marriott.com/hote1s/trave1/csgau-towneplace-suites-auburn/?scid =bb1a189a-fec3-4d19-a255-54ba596febe2. Rates are US\$119 per night for a king studio. To make a reservation online, please use this link: https: //www. marriott. com/meeting-event-hote1s/group-corporate-trave1 /groupCorp.mi?resLinkData=AMS\%5ECSCAU\%60AMSAMSA\%60119.00\%60USD\%60fa7 se\%604\%603/14/19 $\% 603 / 17 / 19 \% 602 / 15 / 19 \& a p p=r e s v 1$ ink\&stop_mobi=yes. TownePlace Suites Auburn offers complimentary breakfast and complimentary parking. This property has high-speed internet an a fitness center.

This property is a shuttle stop for Groome Transportation which services between Auburn and Atlanta International Airport; https://groometransportation. com/auburn/\#gt-auburnsched-1.

The deadline for reservations at this rate is February 15, 2019.

Courtyard by Marriott Auburn (4.2 miles from Mell Classroom Building), 2420 West Pace Boulevard, Auburn, AL 36830 ; (334) 502-0111; https://www. marriott.com/hote1s/trave1/csgca-courtyard-auburn/?scid=bb1a189a -fec3-4d19-a255-54ba596febe2. Rates are US\$109 per night for a double or king room. To make a reservation online, please use this link: www.marriott.com/meeting-event-hote1s/group-corporate-trave1 /groupCorp.mi? resLinkData=Ameri can\%20Mathemati ca 1\%20Soci ety\%5ECSGCA\%60AMAAMAA\%7 CAMAAMAB\%60109.00\%60USD\%60fa 1 se\%604\%603/14/19\%603/17/19\%602/14/19\&app=resv1 ink\&stop _mobi=yes. The Courtyard by Marriott Auburn offers complimentary WiFi and a 24 hour fitness center.

The deadline for reservations at this rate is February 14, 2019.

\section{Housing Warning}

Please beware of aggressive housing bureaus that target potential attendees of a meeting. They are sometimes called "room poachers" or "room-block pirates" and these companies generally position themselves as a meeting's housing bureau, convincing attendees to unknowingly book outside the official room block. They call people who they think will more likely than not attend a meeting and lure them with room rates that are significantly less than the published group rate-for a limited time only. And people who find this offer tempting may hand over their credit card data, believing they have scored a great rate and their housing is a done deal. Unfortunately, this often turns out to be the start of a long, costly nightmare.

Note that some of these room poachers create fake websites on which they represent themselves as the organizers of the meeting and include links to book rooms, etc. The only official website for this meeting is ams.org and one that has the official AMS logo.

These housing bureaus are not affiliated with the American Mathematical Society or any of its meetings, in any way. The AMS would never call anyone to solicit reservations for a meeting. The only way to book a room at a rate negotiated for an AMS Sectional Meeting is via a listing on AMS Sectional Meetings pages or Notices of the AMS. The AMS cannot be responsible for any damages incurred as a result of hotel bookings made with unofficial housing bureaus.

\section{Food Services}

On Campus: There are many dining options on campus within walking distance of the meeting. The hours of the on-campus restaurants vary on the weekends. Please see a complete listing here: https://auburn. campusdish . com/LocationsAndMenus.

Off Campus: Auburn offers many dining options of all types of cuisine. There are many off campus eateries are within half a mile of the meeting, mostly in the College Street/Magnolia Ave area. For more information on dining throughout Auburn as well as general information on visiting Auburn, please visit www . aotouri sm. com

Some of the nearby off-campus dining options include:

BurgerFi, 339 S College Street

burgerfi.com

Newks Eatery, 331 S Gay Street newks. com 
Taziki's, 339 S College Street

tazikiscafe.com

Fuji Sushi Bar, 1499 S College Street

fujisushiau.com

Little Italy Pizzaria, 129 E Magnolia Ave

1ittleitalyau.com

Big Blue Bagel and Deli, 120 N College Street

bigb1uebage 1 andde $1 \mathrm{i}$. com

Cheeburger Cheeburger, 160 N College Street cheeseburger .com

Halftime Bar, 154 N College Street halftimeauburn.com

Auburn Draft House, 165 E Magnolia Avenue auburndrafthouse.com
Hamilton's on Magnolia, 174 E Magnolia Avenue magnolia. hami 1 tonsgroup. com

Zoe's Kitchen, $234 \mathrm{~W}$ Magnolia Avenue zoeskitchen.com

Taco Mama, 149 E Magnolia Avenue tacomamaon 1 ine.com

Tacorita, 138 N College Street tacoritaauburn.com

Ariccia Italian, $241 \mathrm{~S}$ College Street auhcc.com/dining/ariccia-auburn -restaurant

Bizilia's Cafér, 134 N College Street biziliascafe.com

\section{Registration and Meeting Information}

Advance Registration: Advance registration for this meeting opens on January 22, 2019. Advance registration fees will be US $\$ 63$ for AMS members, US $\$ 95$ for nonmembers, and US $\$ 10$ for students, unemployed mathematicians, and emeritus members. Participants may cancel registrations made in advance by emailing mmsb@ams . org. The deadline to cancel is the first day of the meeting.

On-site Information and Registration: The registration desk, AMS book exhibit, and coffee service will be located in the atrium of Mell Classroom Building located at 231 Mell Street. The Invited Address lectures will be located in Science Center Auditorium Building. Special Sessions and Contributed Paper Sessions will take place in Draughton Library-Mell Classroom Buiding and in Parker Hall. Please look for additional information about specific session room locations on the web and in the printed program. For further information on building locations, a campus map is available at https://cws . auburn. edu/map.

The registration desk will be open on Friday, March 15, 1:30 pm to 5:00 pm and Saturday, March 16, 7:30 am to 4:30 $\mathrm{pm}$. The same fees listed above apply for on-site registration and are payable with cash, check or credit card.

\section{Other Activities}

Book Sales: Stop by the on-site AMS bookstore to review the newest publications and take advantage of exhibit discounts and free shipping on all on site orders! AMS and MAA members receive $40 \%$ off list price. Nonmembers receive a $25 \%$ discount. Not a member? Ask a representative about the benefits of AMS membership.

AMS Editorial Activity: An acquisitions editor from the AMS book program will be present to speak with prospective authors. If you have a book project that you wish to discuss with the AMS, please stop by the book exhibit.

Membership Activities During the meeting, stop by the AMS Membership Exhibit to learn about the benefits of AMS Membership. Members receive free shipping on purchases all year long and additional discounts on books purchased at meetings, subscriptions to Notices and Bulletin, discounted registration for world-class meetings and conferences, and more! Complimentary Refreshments will be served courtesy in part by the AMS Membership Department.

\section{Special Needs}

It is the goal of the AMS to ensure that its conferences are accessible to all, regardless of disability. The AMS will strive, unless it is not practicable, to choose venues that are fully accessible to the physically handicapped.

If special needs accommodations are necessary in order for you to participate in an AMS Sectional Meeting, please communicate your needs in advance to the AMS Meetings Department by:

- Registering early for the meeting

- Checking the appropriate box on the registration form, and

- Sending an email request to the AMS Meetings Department at mmsb@ams . org or meet@ams .org .

\section{AMS Policy on a Welcoming Environment}

The AMS strives to ensure that participants in its activities enjoy a welcoming environment. In all its activities, the AMS seeks to foster an atmosphere that encourages the free expression and exchange of ideas. The AMS supports equality of 


\section{MEETINGS \& CONFERENCES}

opportunity and treatment for all participants, regardless of gender, gender identity or expression, race, color, national or ethnic origin, religion or religious belief, age, marital status, sexual orientation, disabilities, or veteran status.

Harassment is a form of misconduct that undermines the integrity of AMS activities and mission.

The AMS will make every effort to maintain an environment that is free of harassment, even though it does not control the behavior of third parties. A commitment to a welcoming environment is expected of all attendees at AMS activities, including mathematicians, students, guests, staff, contractors and exhibitors, and participants in scientific sessions and social events. To this end, the AMS will include a statement concerning its expectations towards maintaining a welcoming environment in registration materials for all its meetings, and has put in place a mechanism for reporting violations. Violations may be reported confidentially and anonymously to 855-282-5703 or at www. mathsoci ety . ethicspoint . com. The reporting mechanism ensures the respect of privacy while alerting the AMS to the situation. Violations may also be brought to the attention of the coordinator for the meeting (who is usually at the meeting registration desk), and that person can provide advice about how to proceed.

For AMS policy statements concerning discrimination and harassment, see the AMS Anti-Harassment Policy at see the www. ams.org/about-us/governance/policy-statements/anti-harassment-policy.

Questions about this welcoming environment policy should be directed to the AMS Secretary at www. ams.org /about-us/governance/sec-contact.

\section{Local Information and Maps}

This meeting will take place on the Auburn University campus. A campus map can be found at https: //cws . auburn . edu/map. Information about Auburn University Department of Mathematics and Statistics can be found at www. auburn . edu/cosam/departments/math/. Please visit the university website at https : //www. auburn. edu for additional information on the campus.

Please watch the AMS website at www . ams . org/meetings/sectiona1/sectiona 1. htm 1 for additional information on this meeting.

\section{Parking}

Parking is available at the Library Parking Deck (5 Roosevelt Dr) whick is adjacent to Mell Classroom Building. A map showing the location of Library Parking Deck can be found here, auburn. edu/admini stration/parking/assets /pdf/CampusParkingMap.pdf.

\section{Travel}

This meeting will take place on the campus of Auburn University located in Auburn, Alabama. Mell Classroom Building is located at 231 Mell Street, Auburn, AL 36849.

By Air:

If you fly to Auburn, you can travel to either the Hartsfield-Jackson Atlanta International Airport (Atlanta, GA) or the Birmingham International Airport (Birmingham, AL). The distance from either airport to Auburn University campus is approximately 100 miles.

Note: There is a one hour time change (Eastern Time to Central Time) when driving across the Georgia-Alabama state line.

Hartsfield-Jackson Atlanta International Airport (www.atlanta-airport.com) is located 100 miles from the Auburn University campus. Average travel time between the airport and campus is 1.5-2 hours.

There is a scheduled ground shuttle service between Harsfield-Jackson and various points in Auburn via Groome Transportation. For more information or to make a reservation, please contact Groome Transportation: www. groometrans portation. com (706)612-1155. The cost for a one-way shuttle service from Hartsfield-Jackson Atlanta International Airport to Auburn University is US $\$ 43.00$. The shuttle schedule can be found here: https: //groometransportation .com/auburn/\#gt-auburnsched-1

For information regarding all other ground transportation from Hartsfield-Jackson Atlanta International Airport, please visit: apps . at 1 . com/Passenger/GroundTransportation/Default . aspx

Birmingham International Airport (BHM), 5900 Airport Hwy, Birmingham, AL 35212, is 114 miles from Auburn University campus. Please visit the airport web site for a list of airlines and lists of cities with daily direct flights to BHM; https://www.f7ybirmingham. com/

Rental cars available at BHM can be found here, https://www. flybirmingham.com/flying-in/ground -transportation/rental-cars/. Taxis, limousine and shuttle services are also available at the Birmingham 
International Airport. For a complete listing, please vistit https://www . flybi rmingham. com/f1ying-in /ground-transportation/1 imous ine-and-shutt 1e-service/. Uber also services BHM. For more information, please visit the Birmingham International Airport website: www. flybi rmingham. com.

ByTrain:

Amtrak does not have a train station in Auburn, the closest train station is in Anniston, AL. One can take a Greyhound bus from the Anniston, AL Amtrak Station to the Opelika, AL Greyhound Station which is about 10 miles from the meeting. Please contact Amtrak at Tel: 800-USA-RAIL, website: www . amtrak . com.

\section{By Bus:}

The closest Greyhound Bus station to Auburn University is located at 300 Columbus Pkwy, Opelika, AL 36801 which is about 10 miles from the meeting. Please contact Greyhound Tel: 1-800-231-2222; Website: www. greyhound.com.

\section{By Car:}

From Hartsfield-Jackson Atlanta International Airport

Depart S Terminal Parkway toward Airport Boulevard and bear right onto Airport Boulevard. Take ramp left for I-285 toward Montgomery. At exit 69, take ramp right for GA-14-SPUR toward South Fulton Parkway. Take ramp right for Buffington Road toward Red Oak. Turn left onto Buffington Road. Turn left onto Estes Drive, and then immediately turn right onto Glen Haven Point. Turn left onto Sable Way. Turn left onto Sable Trail. Turn right onto Sable Way. Turn left onto Sable Bay Point, and then immediately turn right onto Sable Run Road. Turn left onto Buffington Road. Turn right onto Flat Shoals Road. Take ramp left for I-85 S / GA-403 S. Entering Alabama, at exit 57, take ramp right and follow signs for Bent Creek Road. Turn right onto Bent Creek Road. Turn left onto E Glenn Avenue. Turn left onto E Samford Avenue. Turn right onto S Gay Street. Turn left onto Miller Avenue. Road name changes to Roosevelt Drive. Library Parking Deck will be on your right, 5 Roosevelt Drive.

\section{From the Birmingham International Airport:}

Depart toward Messer Airport Hwy and then, keep straight. Take ramp right for I-59 S / I-20 W. At exit 124A, take ramp left for I-65 South toward Montgomery. At exit 171, take ramp right for I-85 North toward Atlanta. At exit 51, take ramp right for US-29 toward Auburn. Bear left onto US-29 / AL-15 / AL-147 / S College Street. Keep straight onto AL-147 / S College St. Turn left onto Roosevelt Drive. Library Parking Deck will be on your right; 5 Roosevelt Drive.

\section{From Montgomery:}

Take I-85 North toward Atlanta. At exit 50, take ramp right toward Auburn Technology Parkway and then turn left onto Cox Rd / CR-12. Bear right onto Wire Rd / CR-137 and then turn right onto W Samford Avenue Turn left onto Mell Street and then turn right onto Roosevelt Drive. Library Parking Deck will be on your left. 5 Roosevelt Drive.

Car Rental: Hertz is the official car rental company for the meeting. To make a reservation accessing our special meeting rates online at www. hertz. com, click on the box "I have a discount," and type in our convention number (CV): CV\#04N30008. You can also call Hertz directly at 800-654-2240 (US and Canada) or 1-405-749-4434 (other countries). At the time of reservation, the meeting rates will be automatically compared to other Hertz rates and you will be quoted the best comparable rate available.

For directions to campus, inquire at your rental car counter.

\section{Local Transportation}

Bus Service: Tiger Transit, servicing Auburn University and Auburn, has several bus stops in the area of the meeting. For a map and schedule, please visit: www . auburn. edu/administration/parking_transit/transit/index.php.

Taxi Service: Licensed, metered taxis are available in Auburn through Tiger Taxi; (334) 444-4444; www . auburntaxi .com.

Taxi Service: Both Lyft and Uber also operate in the Auburn area.

\section{Weather}

The average high temperature in Auburn for March is in the high 60s, Fahrenheit, and the average low is in the mid 40s, Fahrenheit. Visitors should be prepared for inclement weather and check weather forecasts in advance of their arrival. 


\section{MEETINGS \& CONFERENCES}

\section{Social Networking}

Attendees and speakers are encouraged to tweet about the meeting using the hashtag \#AMSmtg.

\section{Information for International Participants}

Visa regulations are continually changing for travel to the United States. Visa applications may take from three to four months to process and require a personal interview, as well as specific personal information. International participants should view the important information about traveling to the US found at https://trave1. state.gov/content /trave1/en . htm1. If you need a preliminary conference invitation in order to secure a visa, please send your request to cro@ams.org.

If you discover you do need a visa, the National Academies website (see above) provides these tips for successful visa applications:

* Visa applicants are expected to provide evidence that they are intending to return to their country of residence. Therefore, applicants should provide proof of "binding" or sufficient ties to their home country or permanent residence abroad. This may include documentation of the following:

- family ties in home country or country of legal permanent residence

- property ownership

- bank accounts

- employment contract or statement from employer stating that the position will continue when the employee returns;

* Visa applications are more likely to be successful if done in a visitor's home country than in a third country;

* Applicants should present their entire trip itinerary, including travel to any countries other than the United States, at the time of their visa application;

* Include a letter of invitation from the meeting organizer or the US host, specifying the subject, location and dates of the activity, and how travel and local expenses will be covered;

* If travel plans will depend on early approval of the visa application, specify this at the time of the application;

* Provide proof of professional scientific and/or educational status (students should provide a university transcript). This list is not to be considered complete. Please visit the websites above for the most up-to-date information.

\section{Honolulu, Hawai'i}

\section{University of Hawai'i at Mānoa}

March 22-24, 2019

Friday - Sunday

\section{Meeting \#1147}

\section{Central Section}

Associate Secretaries: Georgia Benkart and Michel L. Lapidus

Announcement issue of Notices: January 2019

Program first available on AMS website: February 7, 2019

Program issue of electronic Notices: To be announced

Issue of Abstracts: Volume 40, Issue 2

\section{Deadlines}

For organizers: Expired

For abstracts: January 29, 2019

The scientific information listed below may be dated. For the latest information, see www. ams . org/amsmtgs/sectiona1 . htm 1.

\section{Invited Addresses}

Barry Mazur, Harvard University, On the arithmetic of curves (Einstein Public Lecture in Mathematics).

Aaron Naber, Northwestern University, Analysis of geometric nonlinear partial differential equations.

Deanna Needell, University of California, Los Angeles, Simple approaches to complicated data analysis.

Katherine Stange, University of Colorado, Boulder, Title to be announced.

Andrew Suk, University of California, San Diego, On the Erdos-Szekeres convex polygon problem. 


\section{Special Sessions}

If you are volunteering to speak in a Special Session, you should send your abstract as early as possible via the abstract submission form found at http: //www. ams .org/cgi-bin/abstracts/abstract.p1.

Advances in Iwasawa Theory (Code: SS 12A), Frauke Bleher, University of Iowa, Ted Chinburg, University of Pennsylvania, and Robert Harron, University of Hawai'i at Mānoa.

Advances in Mathematical Fluid Mechanics (Code: SS 32A), Kazuo Yamazaki, University of Rochester, and Adam Larios, University of Nebraska - Lincoln.

Algebraic Groups, Galois Cohomology, and Local-Global Principles (Code: SS 3A), Raman Parimala, Emory University, Andrei Rapinchuk, University of Virginia, and Igor Rapinchuk, Michigan State University.

Algebraic Number Theory and Diophantine Equations (Code: SS 20A), Claude Levesque, University of Laval.

Algebraic Points (Code: SS 36A), Barry Mazur and Hector Pasten, Harvard University.

Algebraic and Combinatorial Structures in Knot Theory (Code: SS 9A), Sam Nelson, Claremont McKenna College, Natsumi

Oyamaguchi, Shumei University, and Kanako Oshiro, Sophia University.

Algebraic and Geometric Combinatorics (Code: SS 17A), Andrew Berget, Western Washington University, and Steven

Klee, Seattle University.

Analysis of Nonlinear Geometric Equations (Code: SS 23A), Aaron Naber, Northwestern University, and Richard Bamler, University of California Berkeley.

Analytic and Probabilistic Methods in Convex Geometry (Code: SS 27A), Alexander Koldobsky, University of Missouri, Alexander Litvak, University of Alberta, Dmitry Ryabogin, Kent State University, Vladyslav Yaskin, University of Alberta, and Artem Zvavitch, Kent State University.

Applications of Ultrafilters and Nonstandard Methods (Code: SS 33A), Isaac Goldbring, University of California, Irvine, and Steven Leth, University of Northern Colorado.

Arithmetic Dynamics (Code: SS 29A), Andrew Bridy, Texas A\&M University, Michelle Manes, University of Hawai'i at

Mānoa, and Bianca Thompson, Harvey Mudd College.

Arithmetic Geometry and Its Connections (Code: SS 51A), Laura Capuano, University of Oxford, and Amos Turchet, University of Washington.

Arithmetic and Transcendence of Special Functions and Special Values (Code: SS 56A), Matthew A. Papanikolas, Texas A\&M

University, and Federico Pellarin, Université Jean Monnet, St. Étienne.

Coarse Geometry, Index Theory, and Operator Algebras: Around the Mathematics of John Roe (Code: SS 53A), Erik Guentner,

University of Hawai'i at Mānoa, Nigel Higson, Penn State University, and Rufus Willett, University of Hawai'i at Mānoa.

Coding Theory and Information Theory (Code: SS 39A), Manabu Hagiwara, Chiba University, and James B. Nation,

University of Hawai'i.

Combinatorial and Experimental Methods in Mathematical Phylogeny (Code: SS 47A), Sean Cleary, City College of New York

and the CUNY Graduate Center, and Katherine St. John, Hunter College and the American Museum of Natural History.

Commutative Algebra and its Environs (Code: SS 4A), Olgur Celikbas and Ela Celikbas, West Virginia University, and

Ryo Takahashi, Nagoya University.

Computability, Complexity, and Learning (Code: SS 45A), Achilles A. Beros and Bjørn Kjos-Hanssen, University of

Hawai'i at Mānoa.

Computational and Data-Enabled Sciences (Code: SS 54A), Roummel Marcia, Boaz Ilan, and Suzanne Sindi, University

of California, Merced.

Constructive Aspects of Complex Analysis (Code: SS 7A), Ilia Binder and Michael Yampolsky, University of Toronto, and

Malik Younsi, University of Hawai'i at Mānoa.

Differential Geometry (Code: SS 10A), Vincent B. Bonini, Cal Poly San Luis Obispo, Jie Qing, University of California,

Santa Cruz, and Bogdan D. Suceava, California State University, Fullerton.

Dynamical Systems and Algebraic Combinatorics (Code: SS 57A), Maxim Arnold, University of Texas at Dallas, Jessica

Striker, North Dakota State University, and Nathan Williams, University of Texas at Dallas.

Emerging Connections with Number Theory (Code: SS 43A), Katherine Stange, University of Colorado, Boulder, and

Renate Scheidler, University of Calgary.

Equivariant Homotopy Theory and Trace Methods (Code: SS 58A), Andrew Blumberg, University of Texas, Teena Gerhardt,

Michigan State University, Michael Hill, UCLA, and Michael Mandell, Indiana University.

Factorization and Arithmetic Properties of Integral Domains and Monoids (Code: SS 31A), Scott Chapman, Sam Houston

State University, Jim Coykendall, Clemson University, and Christopher O'Neill, University California, Davis. 


\section{MEETINGS \& CONFERENCES}

Generalizations of Symmetric Spaces (Code: SS 22A), Aloysius Helminck, University of Hawai'i at Mānoa, Vicky Klima, Appalachian State University, Jennifer Schaefer, Dickinson College, and Carmen Wright, Jackson State University.

Geometric Approaches to Mechanics and Control (Code: SS 55A), Monique Chyba, University of Hawai'i at Mānoa, Tomoki

Ohsawa, The University of Texas at Dallas, and Vakhtang Putkaradze, University of Alberta.

Geometry, Analysis, Dynamics and Mathematical Physics on Fractal Spaces (Code: SS 8A), Joe P. Chen, Colgate University,

Lũ(Tim) Hùng, Hawai'i Pacific University, Machiel van Frankenhuijsen, Utah Valley University, and Robert G. Niemeyer, University of the Incarnate Word.

Homotopy Theory (Code: SS 48A), Kyle Ormsby and Angélica Osorno, Reed College.

Interactions between Geometric Measure Theory, PDE, and Harmonic Analysis (Code: SS 41A), Mark Allen, Brigham Young

University, Spencer Becker-Kahn, University of Washington, Max Engelstein, Massachusetts Institute of Technology, and

Mariana Smit Vega Garcia, University of Washington.

Interactions between Noncommutative Algebra and Noncommutative Algebraic Geometry (Code: SS 24A), Garrett Johnson,

North Carolina Central University, Bach Nguyen and Xingting Wang, Temple University, and Daniel Yee, Bradley University.

Lie Theory in the Representations of Groups and Related Structures - dedicated to the memory of Kay Magaard (Code: SS 14A),

Christopher Drupieski, DePaul University, and Julia Pevtsova, University of Washington.

Mapping Class Groups (Code: SS 35A), Asaf Hadari, University of Hawai'i.

Mathematical Analysis of Nonlinear Phenomena (Code: SS 16A), Mimi Dai, University of Illinois at Chicago.

Mathematical Methods and Models in Medicine (Code: SS 19A), Monique Chyba, University of Hawai'i, and Jakob Kotas,

University of Hawai'i and University of Portland.

New Trends in Geometric Measure Theory (Code: SS 37A), Antonio De Rosa, Courant Institute of Mathematical Sciences,

New York University, and Luca Spolaor, Massachusetts Institute of Technology.

New Trends on Variational Calculus and Non-Linear Partial Differential Equations (Code: SS 44A), Craig Cowan, University of Manitoba, Michinori Ishiwata, Osaka University, Abbas Moameni, Carleton University, and Futoshi Takahashi, Osaka City University.

Nonlinear Wave Equations and Applications (Code: SS 42A), Boaz Ilan, University of California, Merced, and Barbara

Prinari, University of Colorado, Colorado Springs.

Numerical Methods for Partial Differential Equations (Code: SS 50A), Evan Gawlik, Michael Holst, and Martin Licht,

University of California, San Diego.

Real and Complex Singularities (Code: SS 34A), Leslie Charles Wilson, University of Hawai'i, Mānoa, Goo Ishikawa,

Hokkaido University, and David Trotman, Aix-Marseille University.

Recent Advances and Applications of Modular Forms (Code: SS 1A), Amanda Folsom, Amherst College, Pavel Guerzhoy,

University of Hawai'i at Mānoa, Masanobu Kaneko, Kyushu University, and Ken Ono, Emory University.

Recent Advances in Lie and Related Algebras and their Representations (Code: SS 28A), Brian D. Boe, University of Georgia,

and Jonathan Kujawa, University of Oklahoma.

Recent Advances in Numerical Methods for PDEs (Code: 2249A), Hengguang Li, Wayne State University, and Sara Pol-

lock, University of Florida.

Recent Advances in Numerical Methods for PDEs (Code: SS 49A), Hengguang Li, Wayne State University, and Sara Pol-

lock, University of Florida.

Recent Developments in Automorphic Forms (Code: SS 2A), Solomon Friedberg, Boston College, and Jayce Getz, Duke University.

Recent Trends in Algebraic Graph Theory (Code: SS 26A), Sebastian Cioaba, University of Delaware, and Shaun Fallat, University of Regina.

SYZ Mirror Symmetry and Enumerative Geometry (Code: SS 11A), Siu Cheong Lau, Boston University, Naichung Leung,

The Chinese University of Hong Kong, and Hsian-Hua Tseng, Ohio State University.

Several Complex Variables (Code: SS 5A), Peter Ebenfelt, University of California, San Diego, John Erik Fornaess,

University of Michigan and Norwegian University of Science and Technology, Ming Xiao, University of California, San

Diego, and Yuan Yuan, Syracuse University.

Spaces of Holomorphic Functions and Their Operators (Code: SS 21A), Mirjana Jovovic and Wayne Smith, University of Hawai'i.

Sparsity, Randomness, and Optimization (Code: SS 15A), Deanna Needell and Jamie Haddock, University of California,

Los Angeles.

Spectral Geometry: The Length and Laplace Spectra of Riemannian Manifolds (Code: SS 25A), Benjamin Linowitz, Oberlin College, and Jeffrey S. Meyer, California State University at San Bernardino. 
Stability and Singularity in Fluid Dynamics (Code: SS 40A), Tristan Buckmaster, Princeton University, Steve Shkoller, University of California, Davis, and Vlad Vicol, Princeton University.

Structural Graph Theory (Code: SS 30A), Zixia Song, University of Central Florida, Martin Rolek, College of William and Mary, and Yue Zhao, University of Central Florida.

The Mathematics of Cryptography (Code: SS 18A), Shahed Sharif, California State University, San Marcos, and Alice Silverberg, University of California, Irvine.

Three-dimensional Floer Theory, Contact Geometry, and Foliations (Code: SS 6A), Joan Licata, Australian National University, and Robert Lipshitz, University of Oregon.

Topics at the Interface of Analysis and Geometry (Code: SS 38A), Alex Austin and Sylvester Eriksson-Bique, University of California, Los Angeles.

Valuations on Algebraic Function Fields and Their Subrings (Code: SS 46A), Ron Brown, University of Hawai'i, Steven Dale Cutkosky, University of Missouri, and Franz-Viktor Kuhlmann, University of Szczecin.

What is Happening in Mathematical Epidemiology? Current Theory, New Methods, and Open Questions (Code: SS 52A), Olivia Prosper, University of Kentucky.

\section{Accommodations}

Participants should make their own arrangements directly with the hotel of their choice. Special discounted rates were negotiated with the hotels listed below. Rates quoted do not include the combined sales tax/hotel occupancy tax of $14.962 \%$. Participants must state that they are with the American Mathematical Society's (AMS) Spring Central and Western Joint Sectional Meeting to receive the discounted rate. The AMS is not responsible for rate changes or for the quality of the accommodations. Hotels have varying cancellation and early checkout penalties; be sure to ask for details.

Pearl Waikiki, 415 Nāhua Street, Honolulu, HI 96815; (808) 922-1616; Reserve a room at https: //pear7waiki ki .reztrip.com/classic/en/specia1_offer?accessCode=AMS19\&action=show\&contro11er $=1$ andings\&1ocale=en\&offer_code=AMS19\&rate_code []=GAMS\&rate_code [] =GAMS\&starting _page $=$ special_offer $\& v r=3$. Rate is US $\$ 110$ plus tax per night for a city view room. Discounted amenity fee of US $\$ 20$ per night plus tax is required. Reservations can also be made by faxing to (808) 922-6223. Some amenities included in the amenity fee are free WiFi access in room and lobby, Blue Ray DVD rental, and coffee and tea service in lobby-7:00 am-9:00 am. This property is located 3.4 miles from campus. A bus stop is located in front of the hotel for easy access to public transportation. Reservations canceled less than 30 days prior to arrival will be charged one night's room rate plus tax. Cancellation and early check-out policies vary and penalties exist at this property; be sure to check when you make your reservation. The deadline for reservations at this reduced rate is February 21, 2019.

Ambassador Hotel Waikiki, 2040 Kuhio Avenue, Honolulu, HI 96815; (808) 941-7777 or fax reservation to (808) 9513939; https://ambassadorwaikiki .com/. Reserve a room at https://ambassadorwaikiki .reztrip.com /c1assic/en/specia1_offer?action=show\&contro 11 er=1andings\& 1 oca 1 e=en\&rate _code\%5B\%5D=AMSS\&rate_code\%5B\%5D=AMSS\&starting_page=specia1_offer\&vr=3. Rate is US $\$ 115$ plus tax per night for a city view room with double beds and a kitchen. Discounted amenity fee of US $\$ 15$ plus tax per night is required and includes wireless internet access, fitness center, and business center. This property is located about 2 miles from campus. A bus stop is located in front of the hotel for easy access to public transportation. Reservation cancellations received after 30 days prior to the arrival will be subject to a cancellation fee of one night room rate and tax for each room cancelled. Cancellation and early check-out policies vary and penalties exist at this property; be sure to check when you make your reservation. The deadline for reservations at this reduced rate is February 21, 2018.

Courtyard by Marriott Waikiki Beach, 400 Royal Hawaiian Avenue, Honolulu, HI 96815; (808) 954-4000; Reserve room at https://www. marriott.com/meeting-event-hotels/group-corporate-trave1/groupCorp . mi ? resLinkData=AMS\%20Spring\%20Centra 1\%20\%26\%20Western\%20Sectiona $1 \% 20$ Meeting\%20 2019\%5EHNLOW\%60AMSAMSA\%7CAMSAMSB\%7CAMSAMSC\%60159. 00-175 . 00\%60USD\%60fa 1 se\%604\%603/21 $/ 19 \% 603 / 25 / 19 \% 602 / 28 / 19 \& a p p=r e s v 1$ i nk\&stop_mobi $i=y e s$. Rates are US $\$ 159$ plus tax per night for a standard room or US $\$ 175$ plus tax per night for a deluxe room. Valet parking rate of US $\$ 25$ plus tax per night. Amenities include free WiFi and fitness center. This property is located about 2 miles from campus. Cancellation and early check-out policies vary and penalties exist at this property; be sure to check when you make your reservation. The deadline for reservations at these reduced rates is February 22, 2019.

Waikiki Beachcomber by Outrigger, 2300 Kalakaua Avenue, Honolulu, HI 96815; (808) 922-4646 phone, (808) 923-4889 fax; https://www.waikikibeachcomber.com/. Make reservations at https://be.synxis . com $/$ ? adu $1 \mathrm{t}=1$ \&ar rive=2019-03-21\&cha $\mathrm{n}=18497$ \&ch $11 \mathrm{~d}=0$ \&cur rency=USD\&depar $\mathrm{t}=2019-03-22$ \&group=190321AMER\&hote1=79163\&1eve1=hote1\&1ocale=en-US\&rooms=1\&sbe_ri=0\&temp 1 ate=GROUP. 


\section{MEETINGS \& CONFERENCES}

Rate is US $\$ 185$ plus tax per night for a single or double city view room. Mandatory resort fee of US $\$ 20$ plus tax per night. Some of the amenities included in the resort fee are unlimited rides on the Waikiki Connection Trolley, WiFi (up to 4 devices), a shopping and discount coupon booklet, complimentary fruit-infused water in the lobby, and 24-hour Business Center access. Valet parking rate of US $\$ 38$ plus tax per night. This property is located 2.2 miles from campus. Cancellation and early check-out policies vary and penalties exist at this property; be sure to check when you make your reservation. The deadline for reservations at this reduced rate is February 5, 2019.

DoubleTree by Hilton Hotel Alana - Waikiki Beach, 1956 Ala Moana Boulevard, Honolulu, HI, 96815; (808) 941-7275; Reserve rooms at https://doubletree.hilton.com/en/dt/groups/personalized/H /HNLKADT-AMS-20190318/index . jhtm1. Rate is US $\$ 188$ plus tax per night for a single or double guest room. Mandatory resort charge of US $\$ 10$ plus tax per night. The group rate includes basic guest internet access, two beach towels, DVD "Now" in-room movie and game rental, coffee and whole fruits in lobby, 22\% discount on Hiking Hawaii Café Mānoa Falls Hike, 10\% off food in Trees Restaurant, local and toll-free calls, heated outdoor pool and deck with Waikiki views, and fitness center. This property is located about 3 miles from the campus. Cancellation and early check-out policies vary and penalties exist at this property; be sure to check when you make your reservation. The deadline for reservations at this reduced rate is February 21, 2019.

Hilton Garden Inn Waikiki Beach, 2330 Kuhio Avenue, Honolulu, HI 96815; (808) 892-1820; https://hi1ton gardeninn. hilton. com/en/gi/groups/personalized/H/HNLKUGI-AM2-20190318/index.jhtm1. Rate is US $\$ 189$ plus tax for a standard room. Mandatory resort fee of US $\$ 25$ per night plus tax. Mandatory porterage fee of US\$8.90 plus tax per night. Some of the amenities included in the resort fee are high speed WiFi access, two Hawaiian juice drinks per day at pool side, and in-room tea kettle with specialty tea. Valet parking rate of US\$39 plus tax per night. This property is located 2.2 miles from campus. Cancellation and early check-out policies vary and penalties exist at this property; be sure to check when you make your reservation. The deadline for reservations at this reduced rate is February 6, 2019.

Hilton Waikiki Beach, 2500 Kuhio Ave, Honolulu, HI 96815; (808) 921-5599; Use the following link for reservations: www. hi 1 ton.com/en/hi/groups/personalized/H/HNLWAHF-AMSSPR-20190321/index.jhtm1, or call in-house reservations directly at (808) 921-5503, or email reservations requests to reservations@hi 1 tonwaikiki hote 1 . com with Group Code: AMSSPR. Rate is US $\$ 190$ plus tax per night for a city view single or double guest room. Some amenities included are city, mountain, or ocean views with private balconies, coffeemakers in rooms, complimentary poolside cabanas, and fitness center. Valet parking rate of US\$35 plus tax per night. Cancellation and early check-out policies vary and penalties exist at this property; be sure to check when you make your reservation. This property is located 3.2 miles from the campus. The deadline for reservations at this reduced rate is February 19, 2019.

\section{Campus Housing}

Participants are urged to reserve campus housing as soon as possible as availability may be limited. They should indicate that they are with the American Mathematical Society's (AMS) Spring Central and Western Joint Sectional Meeting. The AMS is not responsible for any rates or charges, or for the quality of these accommodations.

East-West Center, Housing Office, 1711 East-West Road, Honolulu, HI 96848-1711, Telephone - (808) 944-7805,

Fax - (808) 944-7790, Email - EWC Housing Housing@Eas tWestCenter . org

Go to https: //www. eastwestcenter .org/about-ewc/housing and go to the "Housing Facilities" section for housing options, details on how to reserve a room, list of room types, rate information, cancellation policies, etc. Note that Lincoln Hall has rooms with private bathrooms and more amenities. Hale Mānoa or Hale Kuahine offer more economical accommodations such as student dormitories. The preferred method for making a reservation is by email but participants may also call or fax their requests in.

Other Hotels

The AMS is not holding room blocks at the following properties. These properties are located near the university and are listed for your convenience. The AMS is not responsible for any rates or charges, or for the quality of these accommodations.

- Hilton Hawaiian Village Waikiki Beach Resort, 2005 Kalia Road, Honolulu, Hawai'i 96815, (808) 949-4321

- Hyatt Place Waikiki Beach, 175 Paoakalani Avenue, Honolulu, Hawai'i 96815, (808) 9223861

- Prince Resorts Hawaii, 62-100 Kauna'oa Drive, Kamuela, Hawai'i 96743, (808) 956-1111

- OHANA Waikiki East by Outrigger, 150 Kaiulani Avenue, Honolulu, Hawai'i 96815-3292, (808) 922-5353

- Waikiki Shore by Outrigger, 2161 Kalia Road, Honolulu, Hawai'i 96815, (808) 922-3871

- Waikiki Beach Marriott, 2552 Kalakaua Avenue, Honolulu, Hawai'i 96815, (808) 922-6611

- Sheraton Waikiki, 2255 Kalakaua Avenue, Honolulu, Hawai'i 96815, (808) 922-4422

- Hyatt Regency Waikiki Beach Resort \& Spa, 2424 Kalakaua Avenue, Honolulu, Hawai'i 96815, (808) 923-1234 
- Airbnb near Campus, https: //www . a i rbnb.com/s/2500-Campus-Rd--Honolu1u--HI-96822--USA

\section{Housing Warning}

Please beware of aggressive housing bureaus that target potential attendees of a meeting. They are sometimes called "room poachers" or "room-block pirates" and these companies generally position themselves as a meeting's housing bureau, convincing attendees to unknowingly book outside the official room block. They call people who they think will more likely than not attend a meeting and lure them with room rates that are significantly less than the published group rate-for a limited time only. And people who find this offer tempting may hand over their credit card data, believing they have scored a great rate and their housing is a done deal. Unfortunately, this often turns out to be the start of a long, costly nightmare.

These housing bureaus are not affiliated with the American Mathematical Society or any of its meetings, in any way. The AMS would never call anyone to solicit reservations for a meeting. The only way to book a room at a rate negotiated for an AMS Sectional Meeting is via a listing on AMS Sectional Meetings pages or Notices of the AMS. The AMS cannot be responsible for any damages incurred as a result of hotel bookings made with unofficial housing bureaus.

\section{Food Services}

On Campus: UH Mānoa has a variety of dining options and meal plans to suit your needs and tastes. Dining venues are conveniently located throughout campus and menus range from local plate lunches and bentos to deli sandwiches, international cuisine, vegetarian fare, grill favorites and more. Please see a complete listing here: manoa . hawai $i$. edu/food/. Off Campus: Thanks to innovative chefs and delicious homegrown ingredients, Hawai'i is fast becoming a hot spot for foodies. Fresh and local produce is at your fingertips throughout the region, whether you're splurging on an elegant beachside dinner, feasting on kalua pig and poi at a luau or grabbing a quick al fresco lunch of grilled shrimp and slaw from a food truck.

In many locales, all around the island, you'll find creative eateries taking full advantage of the island's cattle ranches, fresh seafood and hearty vegetables grown in the island's rich, volcanic soil. From traditional Hawaiian cuisine to refined New American fare and a variety of Asian cuisines, the choices are as vast as the landscape. No matter where you eat, a cup of locally grown coffee is the perfect way to finish off any meal. The local beans are so good, you might want to consider a tour and tasting at one of the nearby coffee farms in Kona or Kau.

Search through the many dining options on the island of Hawai'i at https://www.gohawai i.com/islands /oahu/restaurants.

Some of the cafés near campus are:

- Café Morey's, (808) 200-1995, 3106 Monsarrat Avenue, Diamond Head

- Café Miro, (808) 734-2737, 3446 Waialae Avenue

- Sweet E's Café, (808) 737-7771, 1006 Kapahulu Avenue

- Raintree Bakery Coffeehouse, (808) 543-5993, 1110 Pensacola Street

- Kaimana Farm Café, (808) 737-2840, 845 Kapahulu Avenue, Kaimuki

- Café DeLight, (808) 737-0500, 3221 Waialae Avenue

Some of the restaurants near campus are:

- Ba-Le Sandwich Shop, 2445 Campus Road, 808-956-6462

- Subway, 2465 Campus Road, https://www. subway.com

- Tsukuneya, 1442 University Avenue, tsukuneyarobatagri11 . com

- Karai Crab, 901 Hausten Street, karaicrab. com

- LE Crepe Café, 2515 Dole St Richardson Law School Courtyard, www . 1ecrepecafe. com

- Alan Wong's Restaurant, 1857 S King Street Fl 3RD , www . a lanwongs . com

- The Nook Neighborhood Bistro, 1035 University Avenue, www . thenookhono1u1u . com

- Café Kaila, Market City Shopping Center, 2919 Kapiolani Boulevard, www . cafe-kai 1a-hawai i . com

- Da Spot, 2469 S King Street, daspot . net

See a full list at https://www.tripadvi sor.com/Restaurants-g29222-0ahu_Hawai i.htm7.

\section{Registration and Meeting Information}

Advance Registration: Advance registration for this meeting will open on January 22, 2019. Advance registration fees will be US $\$ 63$ for AMS members, US $\$ 95$ for nonmembers, and US $\$ 10$ for students, unemployed mathematicians, and emeritus members. Fees will be payable by cash, check, or credit card. Participants may cancel registrations made in ad- 


\section{MEETINGS \& CONFERENCES}

vance by e-mailing mmsb@ams . org. 100\% refunds will be issued for any advance registrations canceled by the first day of the meeting. After this date, no refunds will be issued.

On-site Information and Registration: The registration desk, AMS book exhibit, and coffee service will be located in the Campus Center. The Invited Addresses will be held in Bilger Hall. Please look for additional information about specific session room locations, particularly for Special and Contributed Paper Sessions, on the web and in the printed program. For further information on building locations, a campus map is available at https://manoa . hawa $i$. edu/ campusmap/.

The registration desk will be open on Friday 8:30 am-4:00 pm, Saturday 8:30 am-4:00 pm, and Sunday 9:00 am-noon. The same fees listed above will apply for on-site registration and will be payable by cash, check, or credit cards.

Membership Activities During the meeting, stop by the AMS Membership Exhibit to learn about the benefits of AMS Membership. Members receive free shipping on purchases all year long and additional discounts on books purchased at meetings, subscriptions to Notices and Bulletin, discounted registration for world-class meetings and conferences, and more!

\section{Other Activities}

Book Sales: Stop by the on-site AMS bookstore to review the newest publications and take advantage of exhibit discounts and free shipping on all on-site orders! AMS and MAA members receive $40 \%$ off list price. Nonmembers receive a $25 \%$ discount. Not a member? Ask a representative about the benefits of AMS membership.

AMS Editorial Activity: An acquisitions editor from the AMS book program will be present to speak with prospective authors. If you have a book project that you wish to discuss with the AMS, please stop by the book exhibit.

Membership Activities: During the meeting, stop by the AMS Membership Exhibit to learn about the benefits of AMS Membership. Members receive free shipping on purchases all year long and additional discounts on books purchased at meetings, subscriptions to Notices and Bulletin, discounted registration for world-class meetings and conferences, and more!

Complimentary Refreshments will be served courtesy in part by the AMS Membership Department.

Einstein Lecture: The Einstein Lecture in 2019 will be held at this meeting in the Kennedy Theatre, on Saturday, March 23, 2019, roughly between 6:15 pm and 7:30 pm. The lecturer will be Barry Mazur, Harvard University. The title of his talk will be On the Arithmetic of Curves. More details TBA.

Satellite Conference: Number Theory Conference, Hawai'i Number Theory 2019 (HINT), March 18-21, 2019. Hawai'i Number Theory 2019 (HINT) will be held at UH Mānoa in the 4 days leading up to the AMS Joint Sectional. It aims to bring together a wide variety of number theorists by hosting 4 concurrently running special sessions. Also featuring 6 plenary speakers, HINT is a great opportunity to extend your 3 days of math to a full week! See more details at math . hawai i . edu/HINT2019/.

\section{Special Needs}

It is the goal of the AMS to ensure that its conferences are accessible to all, regardless of disability. The AMS will strive, unless it is not practicable, to choose venues that are fully accessible to the physically handicapped.

If special needs accommodations are necessary in order for you to participate in an AMS Sectional Meeting, please communicate your needs in advance to the AMS Meetings Department by:

- Registering early for the meeting

- Checking the appropriate box on the registration form, and

- Sending an email request to the AMS Meetings Department at mmsb@ams . org or meet@ams . org.

\section{AMS Policy on aWelcoming Environment}

The AMS strives to ensure that participants in its activities enjoy a welcoming environment. In all its activities, the AMS seeks to foster an atmosphere that encourages the free expression and exchange of ideas. The AMS supports equality of opportunity and treatment for all participants, regardless of gender, gender identity or expression, race, color, national or ethnic origin, religion or religious belief, age, marital status, sexual orientation, disabilities, or veteran status.

Harassment is a form of misconduct that undermines the integrity of AMS activities and mission.

The AMS will make every effort to maintain an environment that is free of harassment, even though it does not control the behavior of third parties. A commitment to a welcoming environment is expected of all attendees at AMS activities, including mathematicians, students, guests, staff, contractors and exhibitors, and participants in scientific sessions and social events. To this end, the AMS will include a statement concerning its expectations towards maintaining a welcoming environment in registration materials for all its meetings, and has put in place a mechanism for reporting violations. Vi- 
olations may be reported confidentially and anonymously to 855-282-5703 or at www . mathsoci ety . ethicspoint. com. The reporting mechanism ensures the respect of privacy while alerting the AMS to the situation. Violations may also be brought to the attention of the coordinator for the meeting (who is usually at the meeting registration desk), and that person can provide advice about how to proceed.

For AMS policy statements concerning discrimination and harassment, see the AMS Anti-Harassment Policy.

Questions about this welcoming environment policy should be directed to the AMS Secretary.

\section{Travel}

By Air: The nearest major airport is the Daniel K. Inouye International Airport (HNL / PHNL), airports . hawa $i$ .gov/hn1/. This airport has international and domestic flights from Honolulu, Hawai'i and is located 9 miles from the center of the University of Hawai'i at Mānoa. See detailed terminal maps at airports.hawai i.gov/hn1 /termina1-maps/.

Bus: See details at https://moovit.com/?from=Danie1\%20K.\%20Inouye\%20Internationa1 \%20A irport\%20 (HNL) \&to=University\%20 of\%20 Hawai i \%20at\%20Manoa\&f $11=21$ $.332115 \_-157.920351 \& t 11=21.293536 \_-157.818379 \&$ cus tomer $I d=4908 \& m e t r o I d=1144 \& 1$ ang=en on how to get to campus from the airport by bus.

Car Rental: Hertz is the official car rental company for the meeting. To make a reservation accessing our special meeting rates online at www. hertz.com, click on the words "Discount code" and type in our convention number (CV): 04N30009. You can also call Hertz directly at 800-654-2240 (US and Canada) or 405-749-4434 (other countries). At the time of your reservation, the meeting rates will be automatically compared to other Hertz rates and you will be quoted the best comparable rate available. Meeting rates include unlimited mileage and are subject to availability. Advance reservations are recommended, blackout dates may apply.

Ground Transportation: See airports.hawai i.gov/hn1/getting-to-from/ground-transportation /the-bus/ on bus service to and from the airport.

Taxicabs: AMPCO Express is the managing contractor of the Airport's Open Taxi System at Daniel K. Inouye International Airport. See details at ai rports . hawai i .gov/hn1/getting-to-from/ground-transportation/taxicab/.

Shuttles: The Roberts Hawaii Express Shuttle provides service to and from the airport daily. See details at ai rports . hawai i.gov/hn1/getting-to-from/ground-transportation/robertshawai ishuttle/.

TNCs: Transportation Network Companies (TNC), Uber and Lyft, are permitted to operate at the Daniel K. Inouye International Airport. The two designated areas on airport property that the TNCs will be able to pick up passengers are located on the second level median curb. One pickup location is at Terminal 1 (formerly the Interisland Terminal) across from Lobby 2 and the other is located at Terminal 2 (formerly the Overseas Terminal) across from Lobby 8. Pick up locations are subject to relocation as construction at the airport proceeds.

\section{Local Information and Maps}

Founded in 1907, the University of Hawai'i at Mānoa (UH Mānoa) is located at 2500 Campus Road, Honolulu, Hawai'i 96822. Its website is https://manoa.hawai i.edu/. See a printable campus map at https://manoa.hawai . edu/wp/wp-content/uploads/2017/09/uhmmap.pdf.

Parking: The University of Hawai'i at Mānoa provides visitors with a variety of parking locations within walking distance to campus. See details at https: //manoa . hawa i i . edu/commuter/vi si tor . php. See parking maps at https: // manoa. hawai i. edu/commuter/maps.html.

Alternate Commuter Services: Visitors to UH Mānoa are encouraged to consider alternatives to driving to campus. See https://manoa. hawai i . edu/commuter/waystomanoa. html for details on these alternatives. See details at https://manoa.hawai i.edu/commuter/transit.htm1 about bus and shuttle services on campus. Those that are staying within a three-mile radius of campus should consider walking, biking, or taking the free Rainbow Shuttle (manoa.hawai i.edu/commuter/rainbowshuttle.htm7).

\section{Weather}

In March, the islands of Hawai'i have an average daytime temperature of $24^{\circ} \mathrm{C} / 75^{\circ} \mathrm{F}$, with highs of $26^{\circ} \mathrm{C} / 79^{\circ} \mathrm{F}$ in the peak of the day. Visitors should be prepared for occasional rain showers.

\section{Social Networking}

Attendees and speakers are encouraged to tweet about the meeting using the hashtag \#AMSmtg. 


\section{MEETINGS \& CONFERENCES}

\section{Information for International Participants}

Visa regulations are continually changing for travel to the United States. Visa applications may take from three to four months to process and require a personal interview, as well as specific personal information. International participants should view the important information about traveling to the US found at https://trave1. state.gov/content /trave1.htm1. If you need a preliminary conference invitation in order to secure a visa, please send your request to meet@ams.org.

If you discover you do need a visa, the Travel.State website (see above) provides these tips for successful visa applications:

1. Visa applicants are expected to provide evidence that they are intending to return to their country of residence. Therefore, applicants should provide proof of "binding" or sufficient ties to their home country or permanent residence abroad. This may include documentation of the following: Family ties in home country or country of legal permanent residence; property ownership bank accounts; and employment contract or statement from employer stating that the position will continue when the employee returns.

2. Visa applications are more likely to be successful if done in a visitor's home country than in a third country.

3. Applicants should include their entire trip itinerary, including travel to any countries other than the United States, at the time of their visa application.

4. Applicants should include a letter of invitation from the meeting organizer or the US host, specifying the subject, location and dates of the activity, and how travel and local expenses will be covered.

5. If travel plans will depend on early approval of the visa application, specify this at the time of the application.

6. Provide proof of professional scientific and/or educational status (students should provide a university transcript).

This list is not to be considered complete. Please visit the website above for the most up-to-date information on how to get a visa.

\section{Inquiries}

For inquiries: meet@ams .org

\section{Hartford, Connecticut}

\section{University of Connecticut Hartford (Hartford Regional Campus)}

\section{April 13-14, 2019}

Saturday - Sunday

\section{Meeting \#1148}

Eastern Section

Associate Secretary: Steven H. Weintraub
Announcement issue of Notices: February 2019 Program first available on AMS website: February 21, 2019 Issue of Abstracts: Volume 40, Issue 2

\section{Deadlines}

For organizers: Expired

For abstracts: February 5, 2019

The scientific information listed below may be dated. For the latest information, see www . ams . org/amsmtgs/sectional . html.

\section{Invited Addresses}

Olivier Bernardi, Brandeis University, Percolation on triangulations and a bijective path to Liouville quantum gravity.

Brian Hall, Notre Dame University, Eigenvalues of random matrices in the general linear group in the large-N limit.

Christina Sormani, Lehman College and CUNY Graduate Center, Compactness Theorems for Sequences of Riemannian Manifolds.

\section{Special Sessions}

If you are volunteering to speak in a Special Session, you should send your abstract as early as possible via the abstract submission form found at www. ams.org/cgi-bin/abstracts/abstract.p1.

Algebraic Number Theory (Code: SS 22A), Harris Daniels, Amherst College, and Alvaro Lozano-Robledo and Erik Wallace, University of Connecticut.

Analysis, Geometry, and PDEs in Non-smooth Metric Spaces (Code: SS 1A), Vyron Vellis, University of Connecticut, Xiaodan Zhou, Worcester Polytechnic Institute, and Scott Zimmerman, University of Connecticut. 


\section{MEETINGS \& CONFERENCES}

Banach Space Theory and Metric Embeddings (Code: SS 11A), Mikhail Ostrovskii, St. John's University, and Beata Randrianantoanina, Miami University.

Chip-firing and Divisor Theory (Code: SS 19A), Caroline Klivans, Brown University, and David Perkinson, Reed College. Cluster Algebras and Related Topics (Code: SS 12A), Emily Gunawan and Ralf Schiffler, University of Connecticut.

Combinatorial Commutative Algebra and Polyhedral Geometry (Code: SS 13A), Elie Alhajjar, US Military Academy, and McCabe Olsen, Ohio State University.

Computability Theory (Code: SS 2A), Damir Dzhafarov and Reed Solomon, University of Connecticut, and Linda Brown

Westrick, Pennsylvania State University.

Convergence of Riemannian Manifolds (Code: SS 17A), Lan-Hsuan Huang and Maree Jaramillo, University of Connecticut, and Christina Sormani, City University of New York Graduate Center and Lehman College.

Discrete Dynamical Systems and Applications (Code: SS 20A), Elliott J. Betrand, Sacred Heart University, and David McArdle, University of Connecticut.

Invariants of Knots, Links, and Low-dimensional Manifolds (Code: SS 15A), Patricia Cahn, Smith College, and Moshe

Cohen and Adam Lowrance, Vassar College.

Knot Theory, the Colored Jones Polynomial, and Khovanov Homology (Code: SS 18A), Adam Giambrone, Elmira College, and Katherine Hall, University of Connecticut.

Mathematical Cryptology (Code: SS 8A), Lubjana Beshaj, United States Military Academy, and Jaime Gutierrez, University of Cantabria, Santander, Spain.

Mathematical Finance (Code: SS 14A), Oleksii Mostovyi, University of Connecticut, Gu Wang, Worcester Polytechnic Institute, and Bin Zhou, University of Connecticut.

Modeling and Qualitative Study of PDEs from Materials Science and Geometry. (Code: SS 6A), Yung-Sze Choi, Changfeng Gui, and Xiaodong Yan, University of Connecticut.

Recent Advances in Structured Matrices and Their Applications (Code: SS 16A), Maxim Derevyagin, University of Connecticut, Olga Holz, University of California, Berkeley, and Vadim Olshevsky, University of Connecticut.

Recent Development of Geometric Analysis and Nonlinear PDEs (Code: SS 3A), Ovidiu Munteanu, Lihan Wang, and Ling Xiao, University of Connecticut.

Representation Theory of Quantum Algebras and Related Topics (Code: SS 10A), Drew Jaramillo, University of Connecticut,

Garrett Johnson, North Carolina Central University, and Margaret Rahmoeller, Roanoke College.

Special Session on Regularity Theory of PDEs and Calculus of Variations on Domains with Rough Boundaries (Code: SS 5A),

Murat Akman, University of Connecticut, and Zihui Zhao, University of Washington.

Special Values of L-functions and Arithmetic Invariants in Families (Code: SS 21A), Ellen Eischen, University of Oregon,

Yifeng Liu, Yale University, Liang Xiao, University of Connecticut, and Wei Zhang, Massachusetts Institute of Technology.

Stochastic Analysis and Related Fields (Code: SS 7A), Fabrice Baudoin, University of Connecticut, and Cheng Ouyang, University of Illinois at Chicago.

Stochastic Processes, Random Walks, and Heat Kernels (Code: SS 4A), Patricia Alonso Ruiz, University of Connecticut, and Phanuel Mariano, Purdue University.

Sub-Riemannian and CR Geometric Analysis (Code: SS 9A), Fabrice Baudoin, University of Connecticut, and Luca Capogna, Worcester Polytechnic Institute. 


\section{MEETINGS \& CONFERENCES}

\section{Quy Nhon City, Vietnam}

\section{Quy Nhon University}

June 10-13,2019

Monday - Thursday

\section{Meeting \#1149}

Associate Secretary: Brian D. Boe

Announcement issue of Notices: April 2019
Program first available on AMS website: To be announced Issue of Abstracts: To be announced

Deadlines

For organizers: Expired

For abstracts: To be announced

The scientific information listed below may be dated. For the latest information, see www . ams . org/amsmtgs/internmtgs . htm 1.

\section{Invited Addresses}

Henry Cohn, Microsoft Research, To be announced.

Robert Guralnick, University of Southern California, To be announced.

Le Tuan Hoa, Hanoi Institute of Mathematics, To be announced.

Nguyen Dong Yen, Hanoi Institute of Mathematics, To be announced.

Zhiwei Yun, Massachusetts Institute of Technology, To be announced.

Nguyen Tien Zung, Toulouse Mathematics Institute, To be announced.

\section{Madison, Wisconsin}

\section{University of Wisconsin-Madison}

\section{September 14-15, 2019}

Saturday - Sunday

\section{Meeting \#1150}

Central Section

Associate Secretary: Georgia Benkart
Announcement issue of Notices: June 2019

Program first available on AMS website: July 23, 2019

Issue of Abstracts: Volume 40, Issue 3

\section{Deadlines}

For organizers: February 14, 2019

For abstracts: July 16, 2019

The scientific information listed below may be dated. For the latest information, see www. ams . org/amsmtgs/sectiona1 . htm 1.

\section{Invited Addresses}

Nathan Dunfield, University of Illinois, Urbana-Champaign, Title to be announced.

Teena Gerhardt, Michigan State University, Title to be announced.

Lauren Williams, University of California, Berkeley, Title to be announced (Erd'ss Memorial Lecture).

\section{Special Sessions}

If you are volunteering to speak in a Special Session, you should send your abstract as early as possible via the abstract submission form found at www. ams.org/cgi-bin/abstracts/abstract. p1.

Association Schemes and Related Topics - in Celebration of J.D.H. Smith's 70th Birthday (Code: SS 8A), Kenneth W. Johnson, Penn State University Abington, and Sung Y. Song, Iowa State University.

Computability Theory in honor of Steffen Lempp's 60th birthday (Code: SS 6A), Joseph S. Miller, Noah D. Schweber, and Mariya I. Soskova, University of Wisconsin-Madison.

Homological and Characteristic $p>0$ Methods in Commutative Algebra (Code: SS 1A), Michael Brown, University of Wisconsin-Madison, and Eric Canton, University of Michigan.

Model Theory (Code: SS 5A), Uri Andrews and Omer Mermelstein, University of Wisconsin-Madison.

Recent Developments in Harmonic Analysis (Code: SS 3A), Theresa Anderson, Purdue University, and Joris Roos, University of Wisconsin-Madison. 


\section{MEETINGS \& CONFERENCES}

Recent Work in the Philosophy of Mathematics (Code: SS 4A), Thomas Drucker, University of Wisconsin-Whitewater, and Dan Sloughter, Furman University.

Several Complex Variables (Code: SS 7A), Hanlong Fang and Xianghong Gong, University of Wisconsin-Madison.

Special Functions and Orthogonal Polynomials (Code: SS 2A), Sarah Post, University of Hawai'i at Mānoa, and Paul Terwilliger, University of Wisconsin-Madison.

Uncertainty Quantification Strategies for Physics Applications (Code: SS 9A), Qin Li, University of Wisconsin-Madison, and Tulin Kaman, University of Arkansas.

\section{Binghamton, New York}

\section{Binghamton University}

October 12-13, 2019

Saturday - Sunday

\section{Meeting \#1151}

Eastern Section

Associate Secretary: Steven H. Weintraub
Announcement issue of Notices: August 2019

Program first available on AMS website: August 29, 2019 Issue of Abstracts: Volume 40, Issue 3

\section{Deadlines}

For organizers: March 12, 2019

For abstracts: August 20, 2019

The scientific information listed below may be dated. For the latest information, see www. ams . org/amsmtgs/sectiona1 . htm1.

\section{Invited Addresses}

Richard Kenyon, Brown University, Title to be announced.

Tony Pantev, University of Pennsylvania, Title to be announced.

Lai-Sang Young, New York University, Title to be announced.

\section{Gainesville, Florida}

\section{University of Florida}

\section{November 2-3,2019}

Saturday - Sunday

\section{Meeting \#1152}

Southeastern Section Associate Secretary: Brian D. Boe

Announcement issue of Notices: September 2019
Program first available on AMS website: September 19, 2019 Issue of Abstracts: Volume 40, Issue 4

\section{Deadlines}

For organizers: April 2, 2019

For abstracts: September 10, 2019

The scientific information listed below may be dated. For the latest information, see www . ams . org/amsmtgs/sectiona1 . htm1.

\section{Invited Addresses}

Jonathan Mattingly, Duke University, To be announced.

Isabella Novik, University of Washington, To be announced.

Eduardo Teixeira, University of Central Florida, To be announced.

\section{Special Sessions}

If you are volunteering to speak in a Special Session, you should send your abstract as early as possible via the abstract submission form found at www. ams.org/cgi-bin/abstracts/abstract.p1.

Fractal Geometry and Dynamical Systems (Code: SS 2A), Mrinal Kanti Roychowdhury, University of Texas Rio Grande Valley.

Geometric and Topological Combinatorics (Code: SS 1A), Bruno Benedetti, University of Miami, Steve Klee, Seattle University, and Isabella Novik, University of Washington. 


\section{MEETINGS \& CONFERENCES}

\section{Riverside, California}

\section{University of California, Riverside}

November 9-10,2019

Saturday - Sunday

\section{Meeting \#1153}

Western Section

Associate Secretary: Michel L. Lapidus

Announcement issue of Notices: September 2019
Program first available on AMS website: September 12, 2019

Issue of Abstracts: Volume 40, Issue 4

\section{Deadlines}

For organizers: April 9, 2019

For abstracts: September 3, 2019

The scientific information listed below may be dated. For the latest information, see www . ams . org/amsmtgs/sectiona1 . htm 1 .

\section{Invited Addresses}

Mohsen Aliabadi, University of Illinois at Chicago, Chicago, IL, A connection between matchings in field extensions and the fundamental theorem of algebra.

Jonathan Novak, University of California, San Diego, Title to be announced.

Anna Skripka, University of New Mexico, Albuquerque, Title to be announced.

\section{Special Sessions}

If you are volunteering to speak in a Special Session, you should send your abstract as early as possible via the abstract submission form found at www . ams.org/cgi-bin/abstracts/abstract.p1.

Inverse Problems (Code: SS 3A), Hanna Makaruk, Los Alamos National Laboratory, and Robert Owczarek, University of New Mexico, Albuquerque and University of New Mexico, Los Alamos.

Random Matrices and Related Structures (Code: SS 2A), Jonathan Novak, University of California, San Diego, and Karl Liechty, De Paul University.

Topics in Operator Theory (Code: SS 1A), Anna Skripka and Maxim Zinchenko, University of New Mexico.

\section{Denver, Colorado}

\section{Colorado Convention Center}

January 15-18, 2020

Wednesday - Saturday

\section{Meeting \#1154}

Joint Mathematics Meetings, including the 126th Annual Meeting of the AMS, 103rd Annual Meeting of the Mathematical Association of America (MAA), annual meetings of the Association for Women in Mathematics (AWM) and the National Association of Mathematicians (NAM), and the winter meeting of the Association for Symbolic Logic (ASL), with

\section{Charlottesville, Virginia}

\section{University of Virginia}

\section{March 13-15, 2020}

Friday - Sunday

Southeastern Section

Associate Secretary: Brian D. Boe sessions contributed by the Society for Industrial and Applied Mathematics (SIAM)

Associate Secretary: Michel L. Lapidus

MAA Associate Secretary: Hortensia Soto

Announcement issue of Notices: October 2019

Program first available on AMS website: November 1, 2019 Issue of Abstracts: To be announced

\section{Deadlines}

For organizers: April 1, 2019

For abstracts: To be announced 


\section{MEETINGS \& CONFERENCES}

\section{Deadlines}

For abstracts: To be announced

For organizers: To be announced

\section{Special Sessions}

If you are volunteering to speak in a Special Session, you should send your abstract as early as possible via the abstract submission form found at www. ams.org/cgi-bin/abstracts/abstract. p1.

Curves, Jacobians, and Abelian Varieties (Code: SS 1A), Andrew Obus, Baruch College (CUNY), Tony Shaska, Oakland University, and Padmavathi Srinivasan, Georgia Institute of Technology.

\section{Medford, Massachusetts}

\section{Tufts University}

March 21-22, 2020

Saturday - Sunday

Eastern Section

Associate Secretary: Steven H. Weintraub

Program first available on AMS website: To be announced
Announcement issue of Notices: To be announced Issue of Abstracts: To be announced

\section{Deadlines}

For organizers: To be announced For abstracts: To be announced

\section{Fresno, California}

\section{California State University, Fresno}

May 2-3,2020

Saturday - Sunday

Western Section

Associate Secretary: Michel L. Lapidus

Announcement issue of Notices: To be announced
Program first available on AMS website: To be announced Issue of Abstracts: To be announced

\section{Deadlines}

For organizers: To be announced For abstracts: To be announced

\section{El Paso, Texas}

\section{University of Texas at El Paso}

\section{September 12-13,2020}

Saturday - Sunday

Central Section

Associate Secretary: Georgia Benkart

Announcement issue of Notices: To be announced
Program first available on AMS website: To be announced Issue of Abstracts: To be announced

\section{Deadlines}

For organizers: To be announced For abstracts: To be announced

\section{State College, Pennsylvania}

Pennsylvania State University, University Park Campus

\section{October 3-4, 2020}

Saturday - Sunday

Eastern Section

Associate Secretary: Steven H. Weintraub Announcement issue of Notices: To be announced Program first available on AMS website: To be announced Issue of Abstracts: To be announced

\section{Deadlines}

For organizers: To be announced For abstracts: To be announced 


\section{MEETINGS \& CONFERENCES}

\section{Salt Lake City, Utah}

\section{University of Utah}

October 24-25, 2020

Saturday - Sunday

Western Section

Associate Secretary: Michel L. Lapidus

Announcement issue of Notices: To be announced
Program first available on AMS website: To be announced Issue of Abstracts: To be announced

\section{Deadlines}

For organizers: To be announced

For abstracts: To be announced

\section{Washington, District of Columbia}

\section{Walter E. Washington Convention Center}

January 6-9, 2021

Wednesday - Saturday

Joint Mathematics Meetings, including the 127th Annual Meeting of the AMS, 104th Annual Meeting of the Mathematical Association of America (MAA), annual meetings of the Association for Women in Mathematics (AWM) and the National Association of Mathematicians (NAM), and the winter meeting of the Association of Symbolic Logic (ASL), with sessions contributed by the Society for Industrial and Applied Mathematics (SIAM).

\section{Grenoble, France}

\section{Université Grenoble Alpes}

July 5-9, 2021

Monday - Friday

Associate Secretary: Michel L. Lapidus

Announcement issue of Notices: To be announced

Program first available on AMS website: To be announced
Associate Secretary: Brian D. Boe

Announcement issue of Notices: October 2020

Program first available on AMS website: November 1, 2020 Issue of Abstracts: To be announced

\section{Deadlines}

For organizers: April 1, 2020

For abstracts: To be announced

\section{Buenos Aires, Argentina}

Issue of Abstracts: To be announced

\section{Deadlines}

For organizers: To be announced

For abstracts: To be announced

Mathematical Congress of the Americas 2021 (MCA2021), the third Mathematical Congress of the Americas (MCA).

The University of Buenos Aires

July 19-23, 2021

Monday - Friday

Associate Secretary: Steven H. Weintraub

Announcement issue of Notices: To be announced

Program first available on AMS website: To be announced

Issue of Abstracts: To be announced

\section{Deadlines}

For organizers: To be announced

For abstracts: To be announced 


\section{MEETINGS \& CONFERENCES}

\section{Omaha, Nebraska}

\section{Creighton University}

October 9-10,2021

Saturday - Sunday

Central Section

Associate Secretary: Georgia Benkart

Announcement issue of Notices: To be announced
Program first available on AMS website: To be announced Issue of Abstracts: To be announced

\section{Deadlines}

For organizers: To be announced For abstracts: To be announced

\section{Seattle, Washington}

\section{Washington State Convention Center and the Sheraton Seattle Hotel}

January 5-8, 2022

Wednesday - Saturday Associate Secretary: Georgia Benkart Announcement issue of Notices: October 2021

Program first available on AMS website: To be announced
Issue of Abstracts: To be announced

\section{Deadlines}

For organizers: To be announced For abstracts: To be announced

\section{Boston, Massachusetts}

John B. Hynes Veterans Memorial Convention Center, Boston Marriott Hotel, and Boston Sheraton Hotel

\section{January 4-7, 2023}

Wednesday - Saturday

Associate Secretary: Steven H. Weintraub

Announcement issue of Notices: October 2022

Program first available on AMS website: To be announced
Issue of Abstracts: To be announced

\section{Deadlines}

For organizers: To be announced

For abstracts: To be announced 


\section{Upcoming Features and Memorial Tributes}

Sticky Particle Dynamics on the Real Line by Ryan Hynd

The Ubiquity of Elliptic Curves by Edray Herber Goins

Predicting the 100-Year Flood to Improve Hurricane Storm

Surge Resilience byTalea Mayo

Assessment of Prediction Algorithms for Ranking Objects by Jacqueline M. Hughes-Oliver

Rudy Horne:The Hidden Figure of Hidden Figures 1968-2017 by Della Dumbaugh

Karen Uhlenbeck and the Calculus of Variations

by Simon Donaldson

The Mathematics of Joan Birman by Dan Margalit

The Life and Pioneering Contributions of an African American Centenarian: Mathematician Katherine G. Johnson

by Johnny L. Houston

The Mathematics of Grace Murray Hopper by Asher Auel

Gertrude M. Cox and Statistical Design by Sharon L. Lohr Industrial Research in Applied Statistics by Taylor Arnold Geometric Measure Theory-Some Recent Applications by TatianaToro

Vladimir Voevodsky by Chuck Weibel 


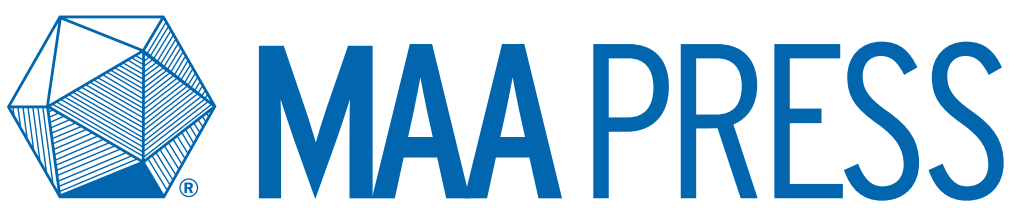

An Imprint

of the

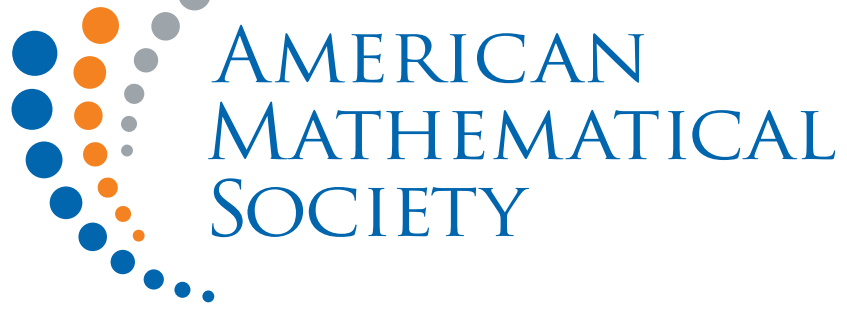

The high-quality mathematics titles and textbooks of the MAA Press are now published as an imprint of the AMS Book Program.

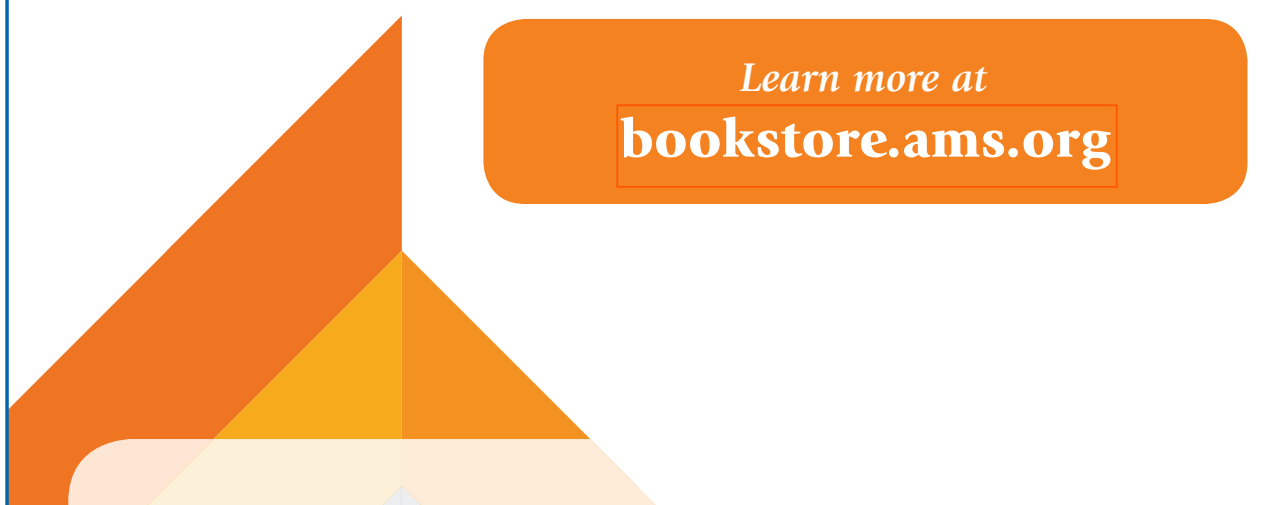

\section{Member discounts for AMS \& MAA members}

- $25 \%$ off the list price of MAA Press titles for both MAA \& AMS members

- $20 \%$ off the list price of most other AMS titles for AMS members

- $10 \%$ off the list price of most other AMS titles for MAA members

AMS MMERECAN

AMS/MaA | rextBooks

An Open Door to

Number Theory

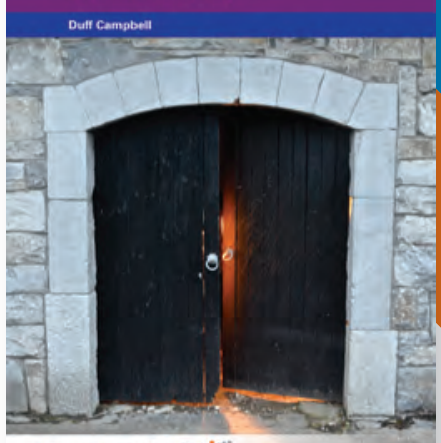

$\triangle$ MAAPRESS $=$ :

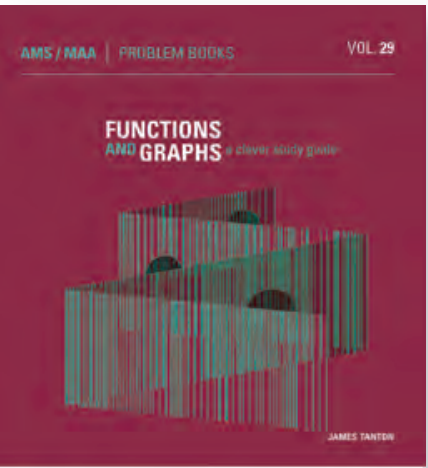

Q MAAPRESS
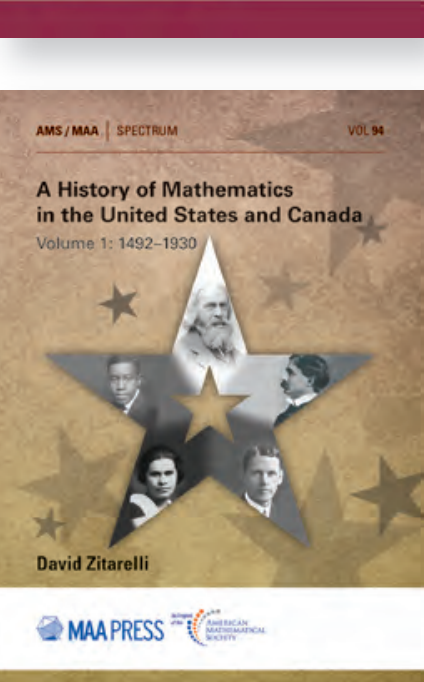
American Mathematical Society

Distribution Center

35 Monticello Place,

Pawtucket, RI 02861 USA
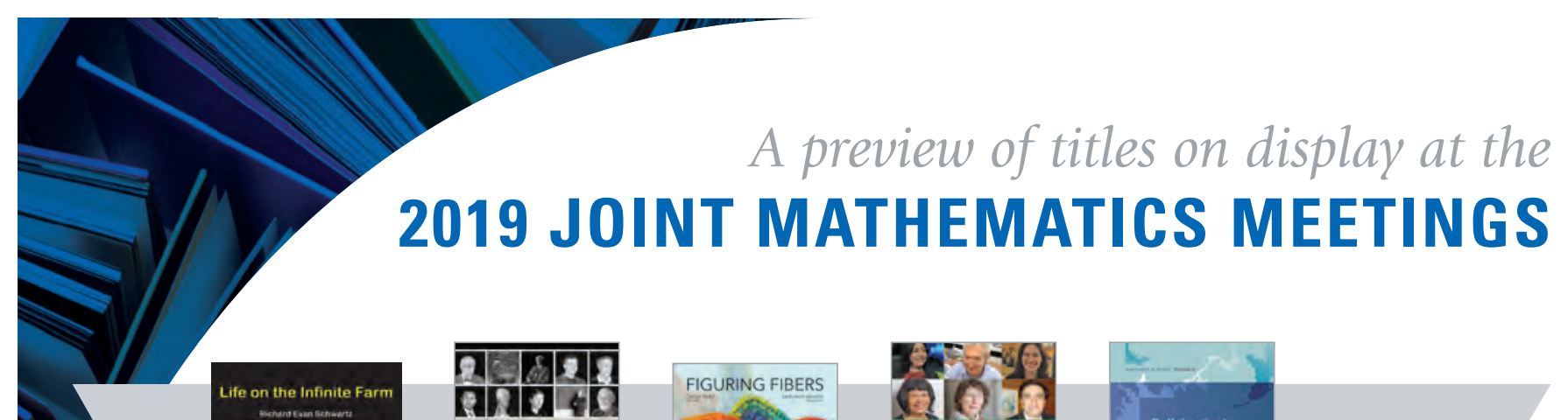
MATHEMATICIANS

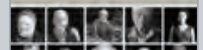

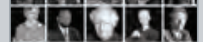

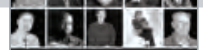

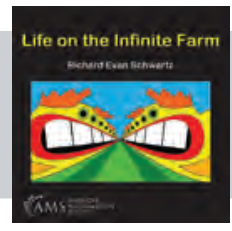

\section{.}

\section{Life on the Infinite Farm}

Richard Evan Schwartz, Brown University, Providence, RI

In Life on the Infinite Farm, mathematician and award-winning children's book author Richard Schwartz teaches about infinity and curved space through stories of whimsical farm animals.

2018; 176 pages; Softcover; ISBN: 978-1-4704-4736-6; List US\$25; MAA members US\$22.50; AMS members US\$20; Order code MBK/115

\section{Mathematicians}

An Outer View of the Inner World

Mariana Cook

Reprinted with corrections by the American Mathematical Society, 2018

This remarkable collection of ninety-two photographic portraits of some of the most impressive mathematicians of our time uses a combination of photographs and short autobiographical texts to illuminate a diverse group of men and women dedicated to the absorbing pursuit of mathematics.

2009; 199 pages; Softcover; ISBN: 978-1-4704-4838-7; List US $\$ 35$; MAA members US $\$ 31.50$; AMS members US\$28; Order code MBK/116

\section{Figuring Fibers}

Carolyne Yackel, Mercer University, Mason, GA, Chief Editor, and sarah-marie belcastro, MathIly, Mathematical Staircase, Inc., Holyoke, MA, and Smith College, Northampton, MA, Assistant Editor

This book transports the reader on a mathematical exploration of fiber arts that is rigorous enough to capture the hearts of mathematicians.

2018; 232 pages; Hardcover; ISBN: 978-1-4704-2931-7; List US\$40; MAA members US\$36; AMS members US $\$ 32$; Order code MBK/117

\section{Limitless Minds}

Interviews with Mathematicians

Anthony Bonato, Ryerson University, Toronto, ON, Canada

Written in an accessible style and enriched by dozens of images, this book offers a rare insight into the minds of mathematicians provided in their own words.

2018; 155 pages; Softcover; ISBN: 978-1-4704-4791-5; List US $\$ 29$; MAA members US $\$ 26.10$; AMS members US $\$ 23.20$; Order code MBK/118

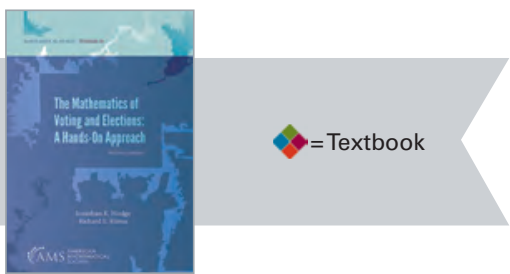

The Mathematics of Voting and Elections: A Hands-On Approach

Second Edition

Jonathan K. Hodge, Grand Valley State University, Allendale, MI, and Richard E. Klima, Appalachian State University, Boone, NC This book is an inquiry-based approach to the mathematics of politics and social choice and aims to give readers who might not normally choose to engage with mathematics recreationally the chance to discover some interesting mathematical ideas from within a familiar context and to see the applicability of mathematics to real-world situations.

Mathematical World, Volume 30; 2018; 238 pages; Softcover; ISBN: 978-1-4704-4287-3; List US\$52; MAA members US $\$ 46.80$; AMS members US $\$ 41.60$; Order code MAWRLD/30

\section{Discover more titles at}

\section{bookstore.ams.org}

\section{AMS \& MAA members will receive 40\% off list price at JMM!}
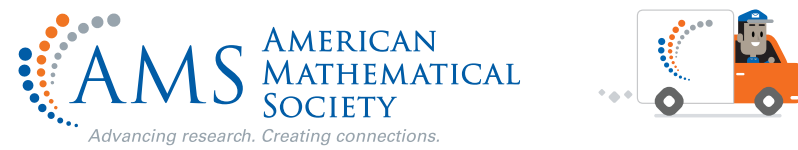

Are you an AMS member? Receive FREE SHIPPING on all of your orders.

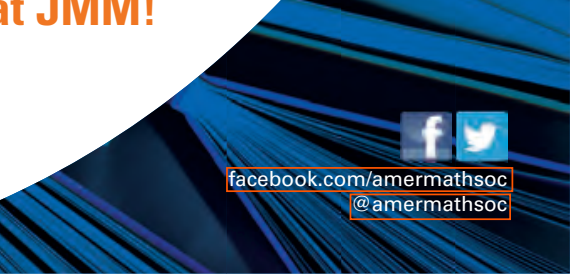

